\title{
Use of the electrocardiogram for prediction of arrhythmia outcome in atrial fibrillation
}

Citation for published version (APA):

Lankveld, T. (2016). Use of the electrocardiogram for prediction of arrhythmia outcome in atrial fibrillation. [Doctoral Thesis, Maastricht University]. Datawyse / Universitaire Pers Maastricht. https://doi.org/10.26481/dis.20161117tl

Document status and date:

Published: 01/01/2016

DOI:

10.26481/dis.20161117t|

Document Version:

Publisher's PDF, also known as Version of record

\section{Please check the document version of this publication:}

- A submitted manuscript is the version of the article upon submission and before peer-review. There can be important differences between the submitted version and the official published version of record.

People interested in the research are advised to contact the author for the final version of the publication, or visit the DOI to the publisher's website.

- The final author version and the galley proof are versions of the publication after peer review.

- The final published version features the final layout of the paper including the volume, issue and page numbers.

Link to publication

\footnotetext{
General rights rights.

- You may freely distribute the URL identifying the publication in the public portal. please follow below link for the End User Agreement:

www.umlib.nl/taverne-license

Take down policy

If you believe that this document breaches copyright please contact us at:

repository@maastrichtuniversity.nl

providing details and we will investigate your claim.
}

Copyright and moral rights for the publications made accessible in the public portal are retained by the authors and/or other copyright owners and it is a condition of accessing publications that users recognise and abide by the legal requirements associated with these

- Users may download and print one copy of any publication from the public portal for the purpose of private study or research.

- You may not further distribute the material or use it for any profit-making activity or commercial gain

If the publication is distributed under the terms of Article $25 \mathrm{fa}$ of the Dutch Copyright Act, indicated by the "Taverne" license above, 


\section{Use of the electrocardiogram for prediction of arrhythmia outcome in atrial fibrillation}

Theo Lankveld 
ISBN: 9789461596154

Print: Datawyse | Universitaire Pers Maastricht 


\title{
Use of the electrocardiogram for prediction of arrhythmia outcome in atrial fibrillation
}

\author{
PROEFSCHRIFT \\ ter verkrijging van de graad van doctor aan de Universiteit Maastricht, \\ op gezag van de Rector Magnificus, Prof. dr. Rianne M. Letschert \\ volgens het besluit van het College van Decanen, \\ in het openbaar te verdedigen \\ op donderdag 17 november 2016 om 14.00 uur \\ door
}

Theodorus Antonius Roelf Lankveld

geboren op 15 maart 1985 te Venray 


\section{Promotores}

Prof. dr. U. Schotten

Prof. dr H.J.G.M. Crijns

\section{Copromotor}

Dr. S. Zeemering

\section{Beoordelingscommissie}

Prof. dr. T. Delhaas (voorzitter)

Prof. dr. A.P. Gorgels

Prof. dr. L. Kappenberger (University of Lausanne, Lausanne, Switzerland)

Prof. dr. F.W. Prinzen

Prof. dr. J.L.R.M. Smeets (Radboud UMC, Nijmegen)

Financial support by the Dutch Heart Foundation for the publication of this thesis is gratefully acknowledged.

Financial support by Stichting Hartsvrienden RESCAR for the publication of this thesis is gratefully appreciated.

Additional financial support was provided by Bayer B.V. and BoehringerIngelheim B.V. and is also gratefully acknowledged. 


\section{Contents}

$\begin{array}{lll}\text { Chapter } 1 & \text { General introduction }\end{array}$

\section{Part I P-wave complexity}

Chapter 2 Increased P-wave complexity in patients with atrial

fibrillation compared to a control population

Part II

The ECG predicting rhythm control strategies

Chapter 3

The ECG as a tool to determine atrial fibrillation complexity

Chapter $4 \quad$ Systematic comparison of non-invasive assessment of atrial fibrillation complexity to predict outcome of pharmacological cardioversion in patients with atrial fibrillation

Chapter $5 \quad$ Systematic analysis of ECG predictors for sinus

rhythm maintenance following electrical

cardioversion

Editorial: Intervention for persistent atrial

fibrillation: prediction of treatment response

Chapter 6 Atrial fibrillation complexity parameters derived

from surface electrocardiograms predict procedural outcome and long-term follow-up of stepwise catheter ablation for atrial fibrillation

Part III

Chapter 7

Chapter 8

Chapter 9
ECG derived complexity and frequency parameters in clinical context

Clinical correlates of non-invasive

electrocardiographic parameters in patients with persistent atrial fibrillation

Dominant frequency predicts sinus rhythm

maintenance after electrical cardioversion for persistent atrial fibrillation in men but not in women General Discussion

Summary 184

Samenvatting 186

Valorisation

Dankwoord 190

Curriculum vitae

List of publications

7 


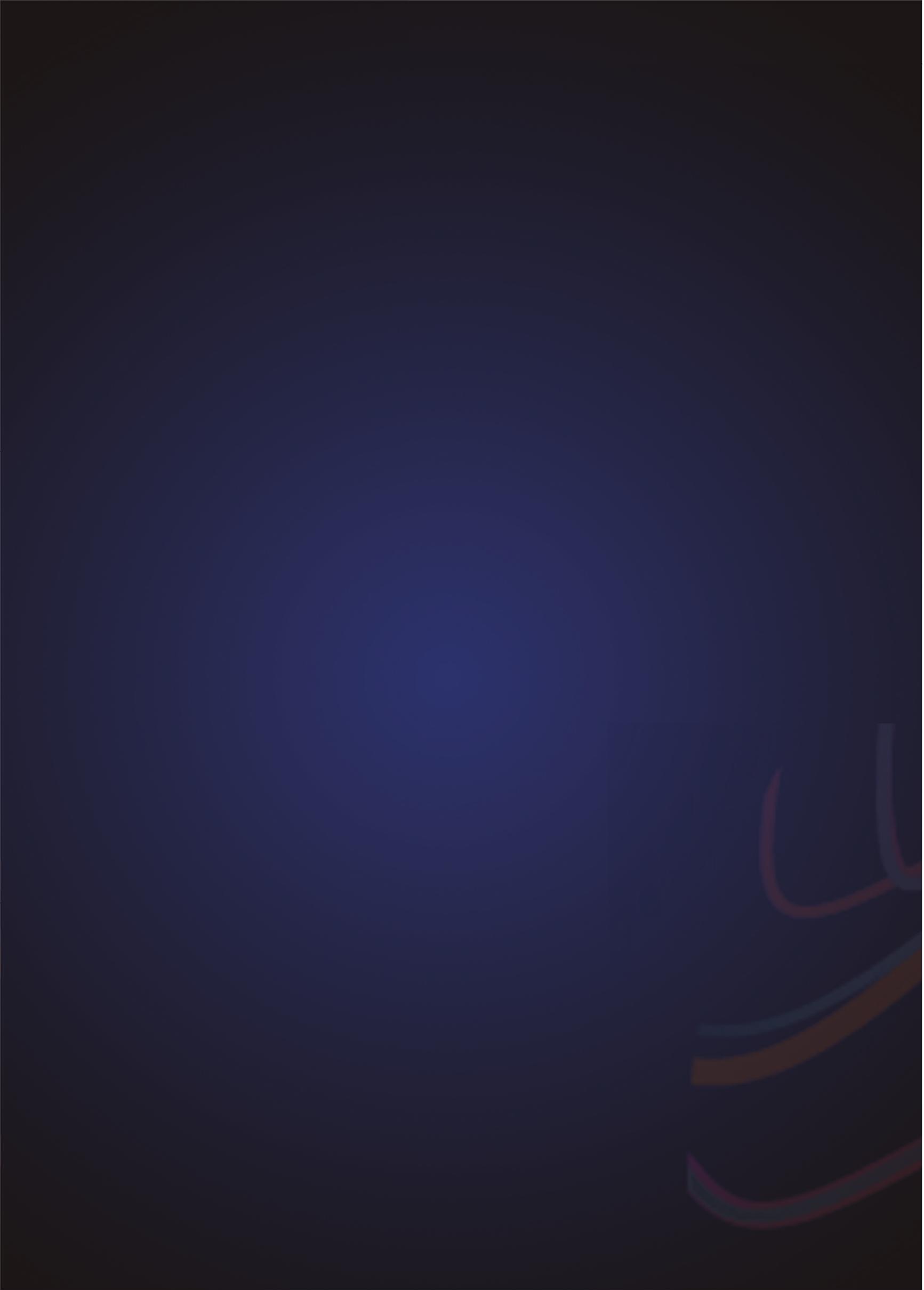




\section{Chapter 1}

General introduction 


\section{Atrial fibrillation}

Atrial fibrillation (AF) is a cardiac arrhythmia characterised by the disorganised activation of the atria. AF is an important risk factor for ischemic stroke and systemic embolism. ${ }^{1}$ Despite extensive and important research in the last decades the exact pathophysiology of AF remains unclear. ${ }^{2}$

Clinically $A F$ is divided into paroxysmal, persistent, long-standing and permanent AF based on duration of the episodes and the decision on rhythm control interventions. ${ }^{3}$ Within one year AF progresses into more sustained forms in $8-22 \%$ of the patients. ${ }^{4-6}$ With increasing $A F$ episode duration $A F$ becomes more stable and rhythm control strategies become less effective. Rhythm control strategies that aim at delaying or prevention of AF progression include anti-arrhythmic drug therapy, catheter ablation (with radiofrequency or cryo-ablation) and surgical ablation, with or without catheter ablation. The latter has the best rhythm outcome ${ }^{7}$, but due to its highly invasive nature is also afflicted with a significant potential complication rate. Anti-arrhtyhmic drugs on the other hand are less effective, may delay appropriate treatment and also are not free of risks, among them ventricular pro-arrhythmia. Identifying the most suitable treatment for an individual patient in an early stage is desirable. So far, there is no prognostic marker or diagnostic test that could identify the most promising therapy for a patient.

\section{Atrial fibrillation and the electrocardiogram: merely diagnosis or more?}

AF is usually recognised on the ECG by the irregular ventricular rhythm without consistent and distinct P-waves. During AF the disorganised atrial activation can be identified by the F-waves on the ECG. These F-waves harbour information about the atrial activation rate and possibly organisation of the atrial electrical activity. ${ }^{8}$ Complexity and frequency parameters derived from these F-waves can distinguish between groups of AF patients (i.e. between patients with paroxysmal and persistent AF and even between patients with persistent and long-standing persistent AF). ${ }^{9-11}$ Several small studies have suggested that these parameters are able to predict rhythm outcome. ${ }^{12-14}$ The problem with these studies is that in most of them only one ECG parameter was studied in a limited population and with limited follow-up. As a consequence, there is no agreement yet on a standardized analysis of F-waves for outcome prediction and none of these parameters is currently used in clinical decision making. Also for the identification of patients at risk for AF development the ECG is hardly used in clinical practice. Several ECG parameters focusing on the P-wave have been studied to identify patients with a high likelihood of developing AF. ${ }^{15-17}$ Unfortunately, these parameters 
provide only limited additional predictive value over easily identifiable clinical predictors. This could be due to the focus on parameters that resemble global rather than regional (left) atrial conduction disturbances.

\section{The aims and outline of this thesis}

The first part of this thesis focuses on patients and individuals during sinus rhythm. The aim of this part is to identify easily obtainable P-wave parameters that help to identify patients at risk for AF. Specifically, in this study P-wave parameters in patients with previous episodes of AF are compared to individuals with no history of AF.

In the second part of this thesis ECG-derived AF complexity and frequency parameters are explored to predict rhythm outcome. Patients with paroxysmal $A F$, persistent $A F$ and long-standing persistent $A F$ are investigated in order to cover the entire spectrum of AF patients. The studies also provide a comparison of these ECG parameters with clinical parameters that are known to predict rhythm outcome. Furthermore, ECG parameters are directly compared to simultaneously measured intracardiac electrograms.

In the third part of this thesis ECG-derived candidate parameters for rhythm outcome prediction identified in the second part of this thesis are investigated in their clinical context. In these studies the effect of age, gender and comorbidities on the ECG parameters for AF complexity and frequency are studied. Also gender-related differences in predictive power of the ECG parameters are investigated.

The long-term objective of this research is to contribute to non-invasive characterisation and classification of AF potentially enabling patient tailored treatment in the future. 


\section{Chapter 1}

\section{References}

1. Wolf PA, Abbott RD, Kannel WB. Atrial fibrillation as an independent risk factor for stroke: the Framingham Study. Stroke; a journal of cerebral circulation Aug 1991;22:983-988.

2. Schotten U, Verheule S, Kirchhof P, Goette A. Pathophysiological mechanisms of atrial fibrillation: a translational appraisal. Physiological reviews Jan 2011;91:265-325.

3. Camm AJ, Kirchhof P, Lip GY, et al. Guidelines for the management of atrial fibrillation: the Task Force for the Management of Atrial Fibrillation of the European Society of Cardiology (ESC). European heart journal Oct 2010;31:2369-2429.

4. Kerr CR, Humphries KH, Talajic M, Klein GJ, Connolly SJ, Green M, Boone J, Sheldon R, Dorian P, Newman D. Progression to chronic atrial fibrillation after the initial diagnosis of paroxysmal atrial fibrillation: results from the Canadian Registry of Atrial Fibrillation. American heart journal Mar 2005;149:489-496.

5. de Vos CB, Pisters R, Nieuwlaat R, Prins MH, Tieleman RG, Coelen RJ, van den Heijkant AC, Allessie MA, Crijns HJ. Progression from paroxysmal to persistent atrial fibrillation clinical correlates and prognosis. Journal of the American College of Cardiology Feb 23 2010;55:725-731.

6. Gianfranchi L, Brignole M, Menozzi C, Lolli G, Bottoni N. Determinants of development of permanent atrial fibrillation and its treatment. Europace : European pacing, arrhythmias, and cardiac electrophysiology : journal of the working groups on cardiac pacing, arrhythmias, and cardiac cellular electrophysiology of the European Society of Cardiology Jan 1999;1:35-39.

7. Pison L, La Meir M, van Opstal J, Blaauw Y, Maessen J, Crijns HJ. Hybrid thoracoscopic surgical and transvenous catheter ablation of atrial fibrillation. Journal of the American College of Cardiology Jul 3 2012;60:54-61.

8. Schotten $U$, Maesen B, Zeemering $S$. The need for standardization of timeand frequency-domain analysis of body surface electrocardiograms for assessment of the atrial fibrillation substrate. Europace : European pacing, arrhythmias, and cardiac electrophysiology : journal of the working groups on cardiac pacing, arrhythmias, and cardiac cellular electrophysiology of the European Society of Cardiology Aug 2012;14:1072-1075.

9. Bollmann A, Kanuru NK, McTeague KK, Walter PF, DeLurgio DB, Langberg JJ. Frequency analysis of human atrial fibrillation using the surface electrocardiogram and its response to ibutilide. The American journal of cardiology Jun 15 1998;81:1439-1445.

10. Bollmann A, Sonne K, Esperer HD, Toepffer I, Langberg JJ, Klein HU. Noninvasive assessment of fibrillatory activity in patients with paroxysmal and persistent atrial fibrillation using the Holter ECG. Cardiovascular research Oct 1999;44:60-66. 
11. Alcaraz R, Sandberg F, Sornmo L, Rieta JJ. Classification of paroxysmal and persistent atrial fibrillation in ambulatory ECG recordings. IEEE transactions on bio-medical engineering May 2011;58:1441-1449.

12. Nault I, Lellouche N, Matsuo S, et al. Clinical value of fibrillatory wave amplitude on surface ECG in patients with persistent atrial fibrillation. Journal of interventional cardiac electrophysiology : an international journal of arrhythmias and pacing Oct 2009;26:11-19.

13. Alcaraz R, Rieta JJ. Time and frequency recurrence analysis of persistent atrial fibrillation after electrical cardioversion. Physiological measurement May 2009;30:479-489.

14. Holmqvist F, Stridh M, Waktare JE, Sornmo L, Olsson SB, Meurling CJ. Atrial fibrillatory rate and sinus rhythm maintenance in patients undergoing cardioversion of persistent atrial fibrillation. European heart journal Sep 2006;27:2201-2207.

15. Magnani JW, Zhu L, Lopez F, Pencina MJ, Agarwal SK, Soliman EZ, Benjamin EJ, Alonso A. P-wave indices and atrial fibrillation: cross-cohort assessments from the Framingham Heart Study (FHS) and Atherosclerosis Risk in Communities (ARIC) study. American heart journal Jan 2015;169:53-61 e51.

16. Schnabel RB, Sullivan LM, Levy D, et al. Development of a risk score for atrial fibrillation (Framingham Heart Study): a community-based cohort study. Lancet Feb 28 2009;373:739-745.

17. Soliman EZ, Prineas RJ, Case LD, Zhang ZM, Goff DC, Jr. Ethnic distribution of ECG predictors of atrial fibrillation and its impact on understanding the ethnic distribution of ischemic stroke in the Atherosclerosis Risk in Communities (ARIC) study. Stroke; a journal of cerebral circulation Apr 2009;40:1204-1211. 


\section{Part I}

$$
\text { P-wave }
$$

complexity 


\section{Chapter 2}

Increased P-wave complexity in

patients with atrial

fibrillation compared to a control population

Theo Lankveld*, Stef Zeemering*, Ernaldo Marcos, Michiel Rienstra, Mark Potse, Hans-Peter Brunner-La Rocca, Matthijs Cluitmans, Paul Volders, Pieter C. Dagnelie, Ronald M. A. Henry, Miranda T. Schram, Simone J. S. Sep, Coen D. A. Stehouwer, Isabelle C. Van Gelder, Harry J. Crijns and Ulrich Schotten

*Contributed equally to this work

Submitted 
Chapter 2

\section{Abstract}

Background: Early detection and treatment of atrial fibrillation (AF) can potentially prevent cardiovascular complications. Unfortunately, current clinical, echocardiographic, and electrocardiographic (ECG) parameters fail to accurately discriminate between patients with and without a history of AF.

Objective: To identify new ECG-derived parameters recorded during sinus rhythm that differentiate between patients with and without AF history.

Methods: Body surface potential mapping was performed for 10 minutes in patients with and without a AF history. We computed several known P-wave parameters on signal averaged $\mathrm{P}$-waves and compared them with newly developed parameters. Furthermore, we investigated which leads showed the highest discriminating power.

Results: We included 123 patients with a history of AF from the AF-RISK Study (62\% male with a mean age of $59 \pm 9$ years) and 137 individuals without AF from the Maastricht Study (62\% male with a mean age of $60 \pm 8$ years). A higher number of discernible peaks in the $\mathrm{P}$-wave and larger $\mathrm{P}$-wave terminal force in lead V1 were independently associated with a history of AF ( $P=0.008$ and $\mathrm{P}=0.026$ respectively). The number of peaks per electrode and their discriminative power were not equally distributed over the body surface. The highest discriminative power was found on the high left posterior side of the thorax.

Conclusion: A higher number of peaks in the $\mathrm{P}$-wave and larger $\mathrm{P}$-wave terminal force in lead V1 can differentiate between patients with and without a history of AF. Especially leads located on the high left posterior side of the body surface show prominent differences. 
P-wave complexity

\section{Introduction}

Atrial fibrillation (AF) is associated with an increase in mortality and morbidity, for example due to an increase in stroke risk. ${ }^{1}$ A large percentage of AF episodes are asymptomatic and therefore remain undetected, but still increase stroke risk. As a consequence, stroke could be the first clinical manifestation of $A F^{2}$ Early detection together with adequate treatment might reduce stroke and other cardiovascular complications., 4 Therefore, intensified monitoring for AF in patients with a high likelihood of developing AF is of great value. Several electrocardiographic (ECG) parameters have been used to predict incident $A F$, with $P$-wave duration (PWD) and PR-interval being the most frequently studied parameters. A longer PWD predicted incident AF in previously undiagnosed patients but, unfortunately does not add predictive power over clinical parameters associated with AF development. ${ }^{5,6}$ The PWD is a surrogate parameter for the total atrial activation time measured on the body surface. Prolongation of the PWD and PR-interval indicate a global conduction slowing but might miss more subtle (regional) conduction disturbances. A parameter that incorporates local irregularities within the Pwave on standard or alternative lead positions might pick up these more subtle regional conduction disturbances and therefore potentially identify patients likely to develop AF more accurately.

The main objective of this study was to compare a wide variety of P-wave parameters in patients with and without a history of AF to identify P-wave parameters associated with prevalent AF. We hypothesised that parameters that are able to detect more subtle conduction slowing are associated with a history of AF. Furthermore, we compared leads from the entire surface of the chest to identify the best lead position to quantify these abnormalities.

\section{Methods}

\section{Patient population}

Patients with a history of AF were recruited from the AF-RISK study. The AFRISK study is an observational, prospective, multicenter study to identify risk factors associated with success of rhythm control therapy in patients with short-lasting symptomatic paroxysmal or persistent AF. Short-lasting paroxysmal AF was defined as AF spontaneously terminating within 7 days, with a total AF history $<2$ years or $<3$ years in case of $\leq 2$ episodes of $\leq 48$ hours per month. Short-lasting persistent AF was defined as AF lasting between 7 days and 1 year with a total AF history $<2$ years. Patients had no contraindications for oral anticoagulation and were $>18$ years of age. Exclusion 
criteria included: postoperative $\mathrm{AF}$, acute coronary syndrome or coronary intervention within the last month, severe valvular disease and a total history of heart failure $>3$ years. For the present substudy we included patients between 40 and 75 years with a complete body surface potential map (BSPM) and we excluded patients with a previous AF ablation, with (partial) atrial pacing during the recordings or poor quality BSPM.

Individuals without AF were recruited from the Maastricht Study. The Maastricht Study is an observational, prospective, population-based cohort study. The rationale and methodology have been described in detail previously. ${ }^{7}$ In brief, the study focuses on the aetiology, pathophysiology, complications, and comorbidities of type 2 diabetes. Individuals aged between 40 and 75 years in whom data was available for analysis were eligible for inclusion. In the present study we only included individuals without a history of $\mathrm{AF}$ and without AF on a recent 24 hour Holter. We only included patients with a complete good quality BSPM, which was not available in all patients for logistical or technical reasons. Both studies were approved by the local ethic committees and all patients provided written informed consent.

\section{BSPM recording and analysis of signal averaged P-waves}

The BSPM was recorded with 184 surface electrodes recorded using a Biosemi ActiveTwo amplifier (Biosemi, Amsterdam, The Netherlands). The Wilson Central Terminal was used as the reference for each electrode. Signals were low-pass filtered at $419 \mathrm{~Hz}$ and digitized with a $2048 \mathrm{~Hz}$ sampling frequency and $0.03 \mu \mathrm{V}$ resolution. A $50 \mathrm{~Hz}$ notch filter was used to suppress power-line interference. No high-pass filter was used during the recording or signal processing. The analyses were performed using custom-made MATLAB software (R2013b, MathWorks Inc. Natick, MA, USA). Signal quality was inspected visually and electrodes with poor contact were removed. After Rpeak detection a patient specific $\mathrm{P}$-wave detection interval preceding the $\mathrm{R}$ peak was selected. All individual P-wave intervals were aligned in time using Pearson correlation, discarding intervals with a correlation coefficient below 0.9. The alignment was performed on the sum of the squared electrode signals to further reduce the effect of noise in individual leads. ${ }^{8}$ For each subject, a mean number of $601 \pm 114$ P-waves were averaged, a median of 12 [5 - 37] Pwaves did not meet the 0.9 correlation threshold. The P-wave parameters were computed on these signal averaged P-waves. An example of this procedure is shown in figure 1. 


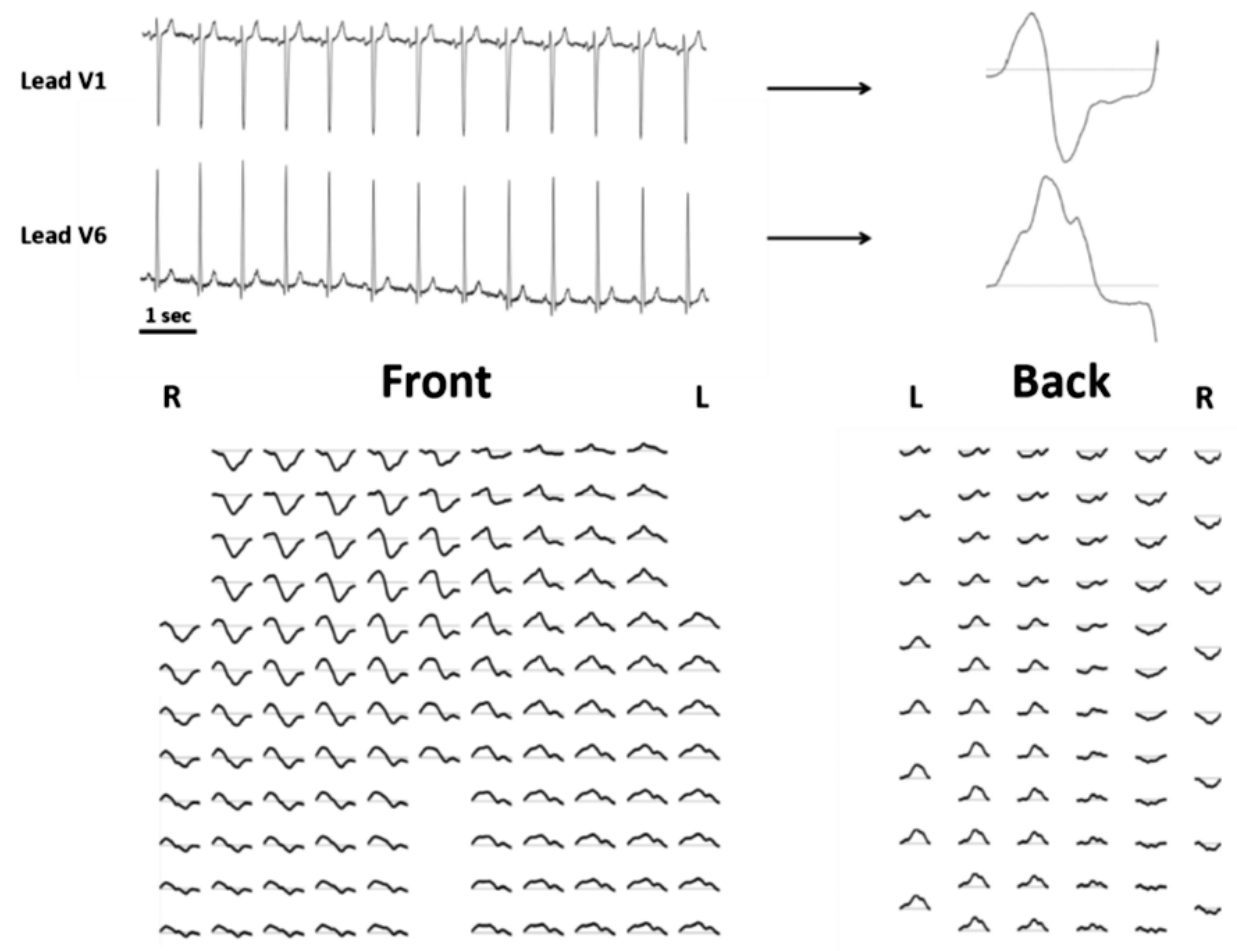

Figure 1: The upper part shows an example of leads V1 and V6 before the averaging procedure (upper left) and after the averaging procedure (upper right). The bottom part shows P-waves of all 184 leads and their distribution over the body surface.

\section{P-wave parameters}

The PWD and PR-interval were computed on the average root mean squared $P$-wave of all included leads. This method incorporates information recorded all over the body surface and provides an accurate estimate of the total atrial activation. $^{9}$ The annotation of the start and end of the unfiltered averaged $\mathrm{P}$ wave was done manually. The area of the averaged P-wave was computed within this interval, as well as the amplitude, which was defined as the difference between the minimum and maximum value of the averaged $\mathrm{P}$-wave. As a measure of P-wave complexity, peaks were detected and counted on the averaged $\mathrm{P}$-wave of each electrode separately. A peak was defined as a positive or negative separate notch in the $\mathrm{P}$-wave with a minimum amplitude of $10 \%$ of a reference amplitude, calculated as the mean amplitude of the average P-wave at each electrode of all patients combined. P-wave complexity, P-wave area and amplitude were computed both as a single value for each 
electrode and as a mean value over the whole body surface. The P-wave terminal force in lead V1 $\left(\mathrm{PTF}_{\mathrm{V} 1}\right)$ is a frequently studied parameter derived from the 12 lead ECG and was computed as the integral of the terminal negative part of the P-wave, only in lead V1.

Orthogonal lead parameters

The $X, Y$ and $Z$ leads were constructed using the BSPM. Three distinct morphological P-wave patterns can be identified using these orthogonal leads. ${ }^{10}$ Type 1 with a positive $P$-wave in leads $X$ and $Y$ and a negative $P$-wave in the $Z$-lead. Type 2 with a positive $P$-wave in the $X$ and $Y$ leads but a biphasic $P$ wave in the Z-lead. Type 3 with positive P-wave in the X-lead and a biphasic Pwave in the $\mathrm{Y}$ and $\mathrm{Z}$-leads. ${ }^{10}$ The $\mathrm{P}$-wave morphology was considered atypical if it did not fit one of the previous definitions. ${ }^{10}$ Furthermore, the absolute areas of the $\mathrm{X}, \mathrm{Y}$ and Z-leads as well as their combined area were calculated.

\section{Electrocardiographic imaging}

To study how differences between P-waves on different locations on the surface of the chest relate to the atrial activation pattern, we compared atrial epicardial activation maps that were created by non-invasive electrocardiographic imaging (ECGI) with the P-wave morphology. ECGI allows to reconstruct potentials, electrograms, and activation isochrones on the epicardium. ${ }^{11,12}$ It can overcome the low spatial resolution of the 12-lead ECG by reconstructing electrograms directly at the epicardium from a much larger set of body-surface electrograms combined with computed tomography (CT)derived information about the heart and body-surface geometries. The relation between electrical heart activity and its projection on the body surface depends on the torso-heart geometry and thoracic conductivities. By carefully reversing this relationship in an inverse procedure, the cardiac source potentials are calculated from the recorded body-surface potentials. After noninvasively reconstructing local epicardial potentials and electrograms, activation isochrone maps are created by defining the moment of activation as the maximum negative slope for each epicardial electrogram. For the present study, we performed ECGI in three additional individuals without a history of AF. Body surface potentials were recorded with the same system as described earlier in this paper, and a CT-scan was performed to create a patient-specific torso-heart geometry. The averaged body-surface P-waves were used to compute epicardial activation isochrones on the atria.

\section{Statistical analysis}


Continuous variables are reported as mean \pm standard deviation or median and range. Comparison between groups was performed using a Student's ttest or Mann-Whitney $U$ test, the latter for non-normally distributed data. Categorical variables are reported as number and percentage and are compared using the chi-square test. Multivariable analysis included the univariate significant parameters and was performed using backward logistic regression analysis. Statistical analyses were performed with IBM SPSS statistics 21 . A P-value of $<0.05$ was considered statistically significant.

\section{Results}

We included 123 patients with a history of AF (62\% male with a mean age of 59 \pm 9 years) and a control group of 137 individuals (62\% male with a mean age of $60 \pm 8$ years) with no known AF history. Baseline characteristics are reported in table 1. Overall patients had a low clinical cardiovascular risk profile, patients without AF more frequently had diabetes mellitus because of the nature of the control cohort. Patients with AF more frequently used beta-blockers, antiarrhythmic drugs and ACE-inhibitors/angiotensin receptor blockers as part of their AF treatment. AF patients had higher left atrial volumes and lower but still normal left ventricular ejection fractions. 


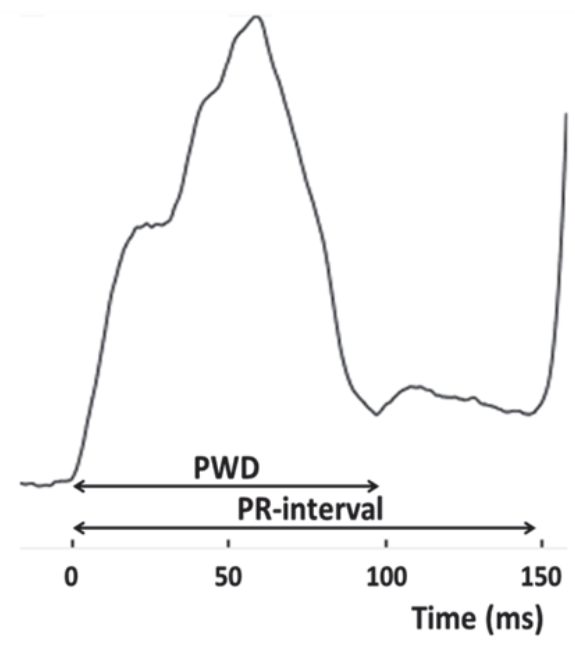

B

$$
\text { Lead V1 }
$$

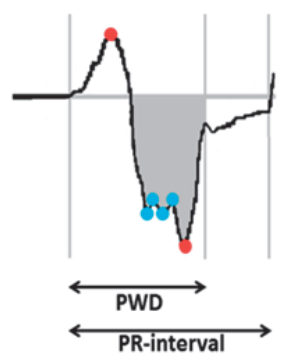

C

Type 1

$\mathbf{X}$

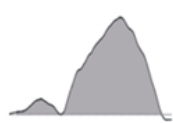

Y

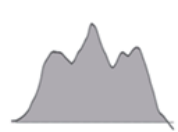

Z

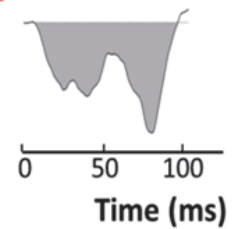

Type 2

$\mathrm{x}$

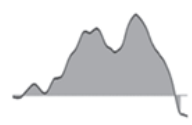

Y

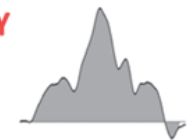

Z

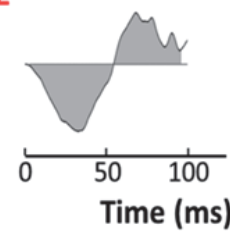

2
Lead V6
A

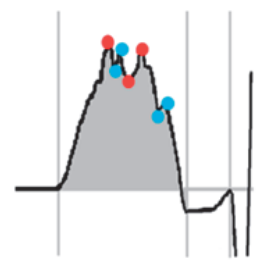

Figure 2: Panel $A$ shows the root mean squared P-wave of an example patient. The overall $\mathrm{P}$-wave duration and $P R$-interval are calculated on this P-wave. Panel B shows an example of leads V1 and V6 and the parameters calculated on the individual leads. The blue and red dots resemble the peaks detected, the amplitude of the red dots exceed the threshold and are therefore recognised as peaks. The grey area in lead V1 shows the P-wave terminal force $V 1$. The grey area in lead V6 resembles the area measured in that lead. Panel $C$ shows the orthogonal leads in three different example patients each with another activation pattern. 


\begin{tabular}{|c|c|c|c|}
\hline & $\begin{array}{c}\text { AF history } \\
\mathrm{N}=123\end{array}$ & $\begin{array}{l}\text { No AF history } \\
\qquad N=137\end{array}$ & P-value \\
\hline Age (y) & $59 \pm 9$ & $60 \pm 8$ & 0.677 \\
\hline Male & $76(62 \%)$ & $85(62 \%)$ & 0.966 \\
\hline Body mass index $\mathrm{Kg} / \mathrm{M}^{2}$ & $27.6 \pm 4.5$ & $27.5 \pm 4.4$ & 0.786 \\
\hline Hypertension & $56(46 \%)$ & 77 (56\%) & 0.086 \\
\hline Diabetes & $6(5 \%)$ & 42 (31\%) & $<0.001$ \\
\hline Stroke & $8(7 \%)$ & $3(2 \%)$ & 0.084 \\
\hline Myocardial infarction & $6(5 \%)$ & $12(9 \%)$ & 0.218 \\
\hline Peripheral artery disease & $3(2 \%)$ & $3(2 \%)$ & 0.894 \\
\hline $\mathrm{CHA}_{2} \mathrm{DS}_{2}$-VASc & $1.5 \pm 1.2$ & $1.8 \pm 1.3$ & 0.054 \\
\hline \multicolumn{4}{|l|}{ AF-type } \\
\hline Paroxysmal & 111 (90\%) & NA & \\
\hline Persistent & $12(10 \%)$ & NA & \\
\hline \multicolumn{4}{|l|}{ Medication } \\
\hline Beta-blocker & $71(58 \%)$ & $23(17 \%)$ & $<0.001$ \\
\hline Diuretics & $20(16 \%)$ & $22(16 \%)$ & 0.965 \\
\hline ACEI/ARB & $54(44 \%)$ & $38(28 \%)$ & 0.006 \\
\hline Calciumantagonist & $11(9 \%)$ & $15(11 \%)$ & 0.590 \\
\hline Statin & $41(33 \%)$ & $50(37 \%)$ & 0.593 \\
\hline AAD & $20(16 \%)$ & $0(0 \%)$ & $<0.001$ \\
\hline \multicolumn{4}{|l|}{ Echo parameters } \\
\hline LVEF (\%) & $58[55-60]$ & 60 [59-62] & $<0.001$ \\
\hline Left atrial volume (mL) & $69 \pm 23$ & $59 \pm 14$ & $<0.001$ \\
\hline
\end{tabular}

Table 1: Baseline characteristics. anti-arrhythmic drug (AAD) , ACE-inhibitors (ACEI), atrial fibrillation (AF), angiotensin receptor blockers (ARB), left ventricular ejection fraction (LVEF). 
Chapter 2

\section{Univariate differences}

Averaged P-wave characteristics

Table 2 shows differences in P-wave parameters between patients with and without a history of AF. Discussed are the P-wave parameters with highly significant differences between the two groups. The averaged P-wave was longer in patients with AF compared to the control population $(p<0.001)$. The PTF $_{\mathrm{V} 1}$ was larger in patients with a history of AF $(p<0.001)$. The mean number of peaks of all surface electrodes was higher in patients with a history of AF $(p<0.001)$.

A longer PWD predicted a history of AF with an area under the receiver operating characteristic (ROC) curve (AUC) of 0.72 . The optimal cut-off, determined from the ROC curve, was $116 \mathrm{~ms}$ and discriminated between AF and SR with a $68 \%$ sensitivity and $69 \%$ specificity. The commonly used cut-off of $120 \mathrm{~ms}$ had a $59 \%$ sensitivity and $77 \%$ specificity. The PTF $\mathrm{V}_{\mathrm{V} 1}$ had an AUC of 0.64 , with a $56 \%$ sensitivity and $72 \%$ specificity at $2,13 \mathrm{mV}^{*} \mathrm{~ms}$. The mean number of peaks over the body surface predicted an AF history with an AUC of 0.65 . The optimal cut-off of a mean of 2.61 peaks had a $63 \%$ sensitivity with a $58 \%$ specificity.

Orthogonal leads

The area of the three orthogonal leads combined was larger in patients with a history of AF ( $p<0.001, A \cup C$ : 0.63). Furthermore, the morphology differed between the two groups. A type 2 morphology was most common in both groups, but a type 1 morphology was more frequently present in patients without a history of AF and a type 3 or atypical P-wave morphology in patients with a previous AF episode $(p=0.017)$.

Spatial heterogeneity

Besides differences in averaged $\mathrm{P}$-wave parameters we also observed regional differences in the discriminative power of the P-wave parameters. Figure 3 shows the spatial distribution of the color-coded AUC values for the discrimination between patients with and without a history of AF based on the number of peaks in the P-wave. The leads located around V5-V6 and cranially of leads V7-V8 show the highest AUC. Lead V6 had an AUC 0.68 with a $63 \%$ sensitivity and $71 \%$ specificity for the optimal cut-off. The lead cranially of lead V7 had an AUC 0.74 with a $65 \%$ sensitivity and a $73 \%$ specificity. 


\begin{tabular}{|c|c|c|c|}
\hline & $\begin{array}{l}\text { AF history } \\
\mathrm{N}=123\end{array}$ & $\begin{array}{l}\text { No AF history } \\
\qquad=137\end{array}$ & P-value \\
\hline Heart rate (bpm) & $57 \pm 8$ & $64 \pm 10$ & $<0.001$ \\
\hline P-waves used (N) & $574 \pm 107$ & $626 \pm 115$ & $<0.001$ \\
\hline \multicolumn{4}{|l|}{ Body surface leads } \\
\hline $\begin{array}{l}\text { P-wave duration } \\
\text { (ms) }\end{array}$ & $123 \pm 13$ & $113 \pm 12$ & $<0.001$ \\
\hline PR-interval (ms) & $176 \pm 25$ & $170 \pm 24$ & 0.065 \\
\hline $\begin{array}{l}\text { P-wave area } \\
\left(\mathrm{mV}^{*} \mathrm{~ms}\right)\end{array}$ & $3.09 \pm 0.79$ & $2.79 \pm 0.66$ & 0.001 \\
\hline $\begin{array}{l}\text { P-wave terminal } \\
\text { force V1 (mV*ms) }\end{array}$ & $2.35 \pm 1.0$ & $1.87 \pm 0.7$ & $<0.001$ \\
\hline Amplitude (mV) & $0.073 \pm 0.015$ & $0.068 \pm 0.014$ & 0.011 \\
\hline $\begin{array}{l}\text { Mean number } \\
\text { peaks }(\mathrm{N})\end{array}$ & $2.93 \pm 0.80$ & $2.51 \pm 0.64$ & $<0.001$ \\
\hline \multicolumn{4}{|l|}{ Orthogonal leads } \\
\hline $\begin{array}{l}\text { P-wave area } \\
\left(\mathrm{mV}^{*} \mathrm{~ms}\right)\end{array}$ & $9.51 \pm 2.40$ & $8.37 \pm 1.86$ & $<0.001$ \\
\hline Morphology & & & 0.017 \\
\hline Type 1 & $17(14 \%)$ & $35(26 \%)$ & \\
\hline Type 2 & 67 (55\%) & $75(55 \%)$ & \\
\hline Type 3/ atypical & 39 (32\%) & $27(20 \%)$ & \\
\hline
\end{tabular}

Table 2: P-wave parameters differences between patients with and without a history of atrial fibrillation (AF). 
Chapter 2
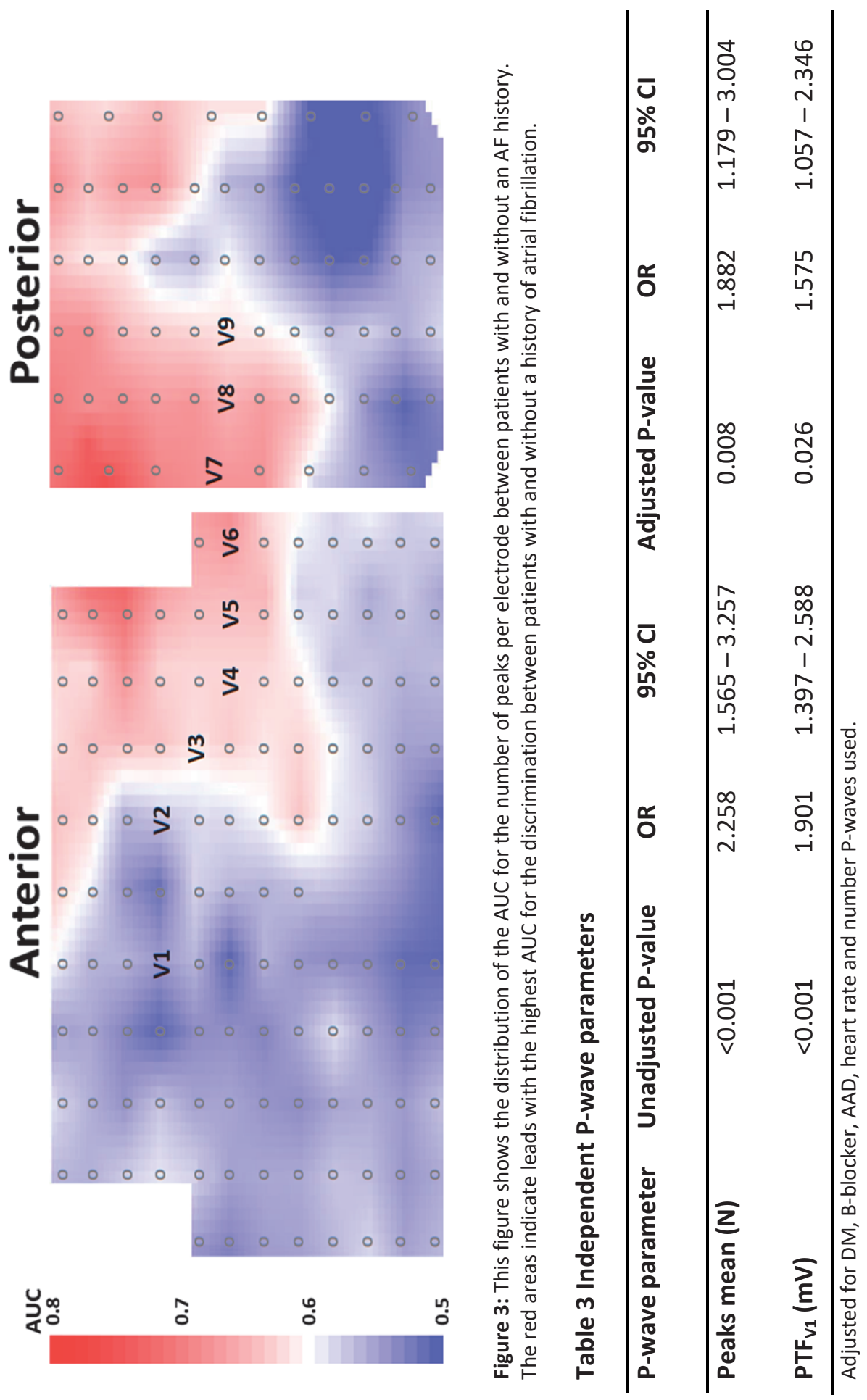


\section{Multivariate analysis of averaged P-wave characteristics}

After correction for diabetes mellitus, hypertension, stroke, heart rate, number of $\mathrm{P}$-waves averaged and medication, a larger mean number of peaks and larger PTF $_{\mathrm{V} 1}$ remained independently associated with a history of AF (table 3). LAV was only calculated in a subset of 205 individuals (103 AF patients). By also correcting for LAV only a larger PTF $_{\mathrm{V} 1}$ remained independently associated with $\operatorname{AF}(p=0.02)$, mean number of peaks was borderline significant $(p=0.05)$.

\section{Epicardial activation isochrones}

ECGI in three patients consistently showed epicardial activation starting at the high right atrium (figure 4). The latest atrial activation was found on the left atrial lateral wall. The figure also shows the surface P-waves of leads V1, V6 and V8. In all three examples the amplitude of the P-wave during right atrial activation was large in lead V1, smaller in lead V6 and hardly distinguishable from the zero-line in lead V8. Vice-versa, during left atrial activation the amplitude was relatively small in lead V1 and largest in V8, suggesting that the lead V8 primarily reflects late left atrial activation.

\section{Discussion}

We demonstrate that a higher number of peaks in the P-wave and a larger PTF $_{\mathrm{V} 1}$ are independently associated with a history of AF. Importantly, not all surface locations harboured the same predictive information. Especially leads located on the back cranial to leads V7-V8 contain information discriminating between patients with or without a history of AF.

\section{P-wave peak detection}

We showed that patients with a history of AF have more complex P-waves than patients without known AF. Although one small previous study has shown more fractionated P-waves in patients with a history of $A F,{ }^{13}$ our understanding of the pathophysiological meaning of complex P-waves is very limited. A previous study showed more fractionated P-waves in patients with more atrial fatty infiltrations. ${ }^{14}$ Pericardial atrial fat is independently associated with $\mathrm{AF}$ and can also promote atrial fibrosis. ${ }^{15,16}$ Atrial fibrosis results in heterogeneities in conduction favouring re-entry and perpetuation of AF. Studies on the presence and origin of notches within the QRS complex found that these notched or fragmented QRS complexes indeed can be caused by regional activation delays. ${ }^{17}$ Therefore, a P-wave with a more complex pattern 


\section{Chapter 2}

illustrated by more peaks might reflect a higher degree of (regional) atrial conduction disturbances. Atrial conduction disturbances could explain the higher likelihood of AF.

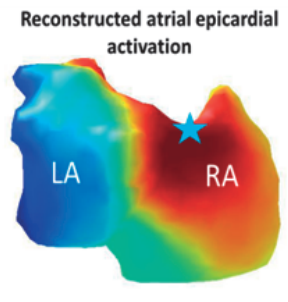

Reconstructed atrial epicardial activation

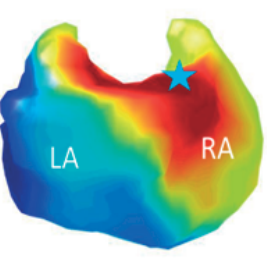

Reconstructed atrial epicardial activation

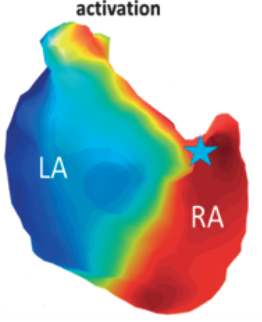

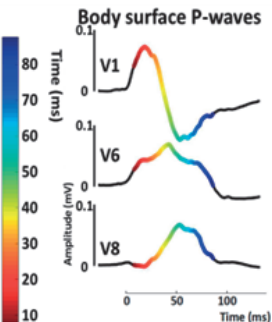

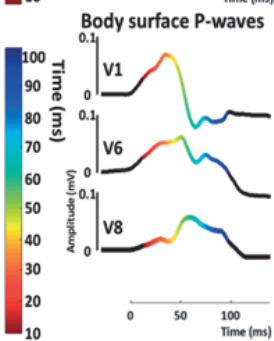

Body surface P-waves
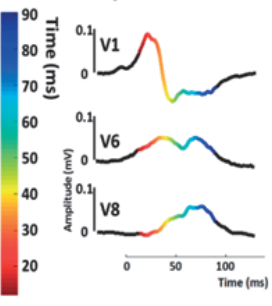

PA view lead V8

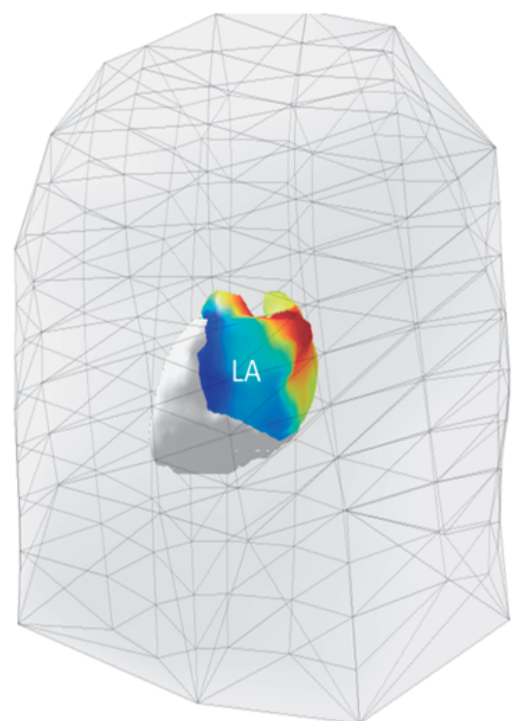

Figure 4: This figure shows the epicardial activation of three individuals without a history of atrial fibrillation. The left panel shows the reconstructed atrial epicardial activation and corresponding surface P-waves of leads V1, V6 and V8. The atrial activation starts at the asterisk. The right panel shows the posterior-anterior view from lead V8 of the second individual. Predominately the left atrium is located towards the electrode. 


\section{Added diagnostic value of leads besides the standard 12-lead ECG}

A potential advantage of a BSPM over a standard 12-lead ECG is the additional spatial information that can be used to examine which regions of the chest surface differs most between patients. For example, although we showed that the mean number of peaks from all electrodes combined identified patients with a previous AF episode, the predictive performance did not outperform the PWD. However, leads located cranially to the leads V7-V8 performed better in the identification of patients likely to have experienced AF. Recent publications showed that atrial fibrosis, epicardial fat, conduction slowing, and low voltage areas are especially present in the left atrial posterior wall in AF. ${ }^{18-20}$ Our ECGI data demonstrate that P-waves in lead V8 predominantly reflect left atrial activation. This may well explain the discriminating power of the number of peaks in this body surface location. Some previous studies also found additional value of leads located on the back. ${ }^{21,22}$ However, there is a need for prospective studies on the predictive value of leads located on the back or the entire BSPM for incident AF.

\section{Comparison with classic P-wave parameters}

PWD is the most frequently studied P-wave parameter. As already demonstrated by previous studies, we confirm that a longer unfiltered signal averaged PWD identifies patients with an AF history. ${ }^{23}$ However, we used information from leads located all over the body surface which might have increased sensitivity and specificity. In longitudinal studies, a longer PWD also predicts new onset AF. ${ }^{5}{ }^{24}$ However, the hazard ratios for AF development were small and provided no contribution beyond traditional risk factors for AF development. ${ }^{5}$ Another frequently studied parameter is the PR-interval. A long PR-interval is associated with development of AF. ${ }^{25}$ Surprisingly we found no significant difference in PR duration between patients with and without AF.

PTF $_{\mathrm{V}_{1}}$ is frequently studied to identify left atrial dilatation in standard 12-lead ECGs. Next to left atrial dilatation, a larger PTF ${ }_{\mathrm{V} 1}$ can also be the result of an increase in atrial conduction delay. ${ }^{26}$ Previous studies showed its association with stroke and AF. ${ }^{27,}{ }^{28}$ Also in the present study we showed that a higher PTF $_{\mathrm{v} 1}$ is independently associated with a history of AF emphasizing the importance of left atrial parameters.

\section{Orthogonal leads}

Orthogonal leads can be used to describe the P-wave morphology in three dimensions. ${ }^{10}$ In our study a type 1 ECG was more common in patients without 
AF history whereas a type 3 or atypical ECG morphology was more common in patients with a previous AF episode. A previous study showed that an abnormal P-wave ECG (other than type 1) was associated with an increased the risk for incident $A F^{10,29}$ This could be explained by a higher degree of interatrial conduction defects. Interatrial conduction over Bachmann's bundle only, without contribution of the posterior fibres, results in an anterior to posterior activation of the left atrium and a type 2 morphology. A more advanced interatrial block occurs when conduction over Bachmann's bundle is also interrupted which will result in left atrial activation by the inferior interatrial pathway and in turn in a type 3 morphology. ${ }^{26}$

\section{Clinical implications and future directions}

As demonstrated by this and other studies characteristics of the P-wave might be useful to discriminate between patients with a known history of AF and patients without AF even in a population with a low cardiovascular risk profile. However, the main clinical question is whether patients likely to develop AF can be identified using this technique, possibly in combination with clinical characteristics and serum biomarkers. Identification of patients at risk for AF may result in more intense monitoring for AF episodes and thus potentially in earlier initiation of antithrombotic therapy. Our study also clearly indicates that leads representing the left atrium and that are not included in the standard 12-lead ECG might be of added value for the identification of these patients over a conventional 12-lead ECG. These alternative P-wave parameters and leads should be used to test its power to predict AF development in future studies. Furthermore, it would be worthwhile to study their predictive performances for either AF progression or response to treatment in a population with paroxysmal AF.

\section{Limitations}

A limitation is the need for signal-averaging and recording systems with a high sampling frequency to acquire the $\mathrm{P}$-waves suitable for the peak detection. The recording of an averaged ECG is more time-consuming and therefore not frequently used in daily clinical practice. However, this technique might be worthwhile exploring if it shows to provide a more accurate identification of individuals who will develop AF over conventional 10 seconds 12-lead ECGs. We used 184-lead BSPMs, this technique obviously requires longer and more dedicated preparation than a 12-lead ECG.

We used individuals without a known AF history and without AF on a 24 hour Holter monitor as a control population. Nonetheless, these controls might 
have experienced undocumented asymptomatic AF episodes. Furthermore, the cases and controls in this study are from two different cohorts which might have introduced a selection bias. The control study mainly focuses on diabetes mellitus explaining the high percentage of patients with diabetes mellitus in the control population.

\section{Conclusions}

A higher irregularity of the P-wave, measured by the number of peaks within the P-wave, identifies patients with a previous history of AF. Particularly the number of peaks measured in leads located cranially of leads V7-V8 seem useful to identify these patients and outperform commonly used parameters such as the PWD. 


\section{Chapter 2}

\section{References}

1. Vermond RA, Geelhoed B, Verweij N, Tieleman RG, Van der Harst P, Hillege HL, Van Gilst WH, Van Gelder IC, Rienstra M. Incidence of Atrial Fibrillation and Relationship With Cardiovascular Events, Heart Failure, and Mortality: A Community-Based Study From the Netherlands. Journal of the American College of Cardiology 2015;66:1000-1007.

2. Healey JS, Connolly SJ, Gold MR, et al. Subclinical atrial fibrillation and the risk of stroke. The New England Journal of Medicine 2012;366:120-129.

3. Nattel S, Guasch E, Savelieva I, et al. Early management of atrial fibrillation to prevent cardiovascular complications. European Heart Journal 2014;35:14481456.

4. Kirchhof P, Breithardt G, Camm AJ, Crijns HJ, Kuck KH, Vardas P, Wegscheider $\mathrm{K}$. Improving outcomes in patients with atrial fibrillation: rationale and design of the Early treatment of Atrial fibrillation for Stroke prevention Trial. American Heart Journal 2013;166:442-448.

5. Magnani JW, Zhu L, Lopez F, Pencina MJ, Agarwal SK, Soliman EZ, Benjamin EJ, Alonso A. P-wave indices and atrial fibrillation: cross-cohort assessments from the Framingham Heart Study (FHS) and Atherosclerosis Risk in Communities (ARIC) study. American Heart Journal 2015;169:53-61 e51.

6. Alonso A, Krijthe BP, Aspelund T, et al. Simple risk model predicts incidence of atrial fibrillation in a racially and geographically diverse population: the CHARGE-AF consortium. Journal of the American Heart Association 2013;2:e000102.

7. Schram MT, Sep SJ, van der Kallen CJ, Dagnelie PC, Koster A, Schaper N, Henry RM, Stehouwer CD. The Maastricht Study: an extensive phenotyping study on determinants of type 2 diabetes, its complications and its comorbidities. European Journal of Epidemiology 2014;29:439-451.

8. Potse M, Lankveld TA, Zeemering S, Dagnelie PC, Stehouwer CD, Henry RM, Linnenbank AC, Kuijpers $\mathrm{NH}$, Schotten U. P-wave complexity in normal subjects and computer models. Journal of Electrocardiology 2016;49:545-53.

9. Gozolits S, Fischer G, Berger T, Hanser F, Abou-Harb M, Tilg B, Pachinger O, Hintringer F, Roithinger FX. Global P wave duration on the 65-lead ECG: singlesite and dual-site pacing in the structurally normal human atrium. Journal of Cardiovascular Electrophysiology 2002;13:1240-1245.

10. Holmqvist F, Platonov PG, McNitt S, Polonsky S, Carlson J, Zareba W, Moss AJ. Abnormal P-wave morphology is a predictor of atrial fibrillation development and cardiac death in MADIT II patients. Annals of Noninvasive Electrocardiology 2010;15:63-72.

11. Cluitmans MJ, Peeters RL, Westra RL, Volders PG. Noninvasive reconstruction of cardiac electrical activity: update on current methods, applications and challenges. Netherlands Heart Journal 2015;23:301-311. 
12. Ramanathan C, Ghanem RN, Jia P, Ryu K, Rudy Y. Noninvasive electrocardiographic imaging for cardiac electrophysiology and arrhythmia. Nature Medicine 2004;10:422-428.

13. Censi F, Ricci C, Calcagnini G, Triventi M, Ricci RP, Santini M, Grammatico A, Bartolini P. Time-domain and morphological analysis of the $\mathrm{P}$ wave. Part II: effects of atrial pacing on P-wave features. Pacing and Clinical Electrophysiology 2008;31:935-942.

14. Murthy S, Rizzi P, Mewton N, Strauss DG, Liu CY, Volpe GJ, Marchlinski FE, Spooner P, Berger RD, Kellman P, Lima JA, Tereshchenko LG. Number of Pwave fragmentations on P-SAECG correlates with infiltrated atrial fat. Annals of Noninvasive Electrocardiology 2014;19:114-121.

15. Al Chekakie MO, Welles CC, Metoyer R, Ibrahim A, Shapira AR, Cytron J, Santucci P, Wilber DJ, Akar JG. Pericardial fat is independently associated with human atrial fibrillation. Journal of the American College of Cardiology 2010;56:784-788.

16. Venteclef N, Guglielmi V, Balse E, Gaborit B, Cotillard A, Atassi F, Amour J, Leprince $P$, Dutour A, Clement K, Hatem SN. Human epicardial adipose tissue induces fibrosis of the atrial myocardium through the secretion of adipofibrokines. European Heart Journal 2015;36:795-805a.

17. Morita H, Kusano KF, Miura D, Nagase S, Nakamura K, Morita ST, Ohe T, Zipes $\mathrm{DP}, \mathrm{Wu}$ J. Fragmented QRS as a marker of conduction abnormality and a predictor of prognosis of Brugada syndrome. Circulation 2008;118:1697-1704.

18. Cochet $\mathrm{H}$, Mouries $\mathrm{A}$, Nivet $\mathrm{H}$, et al. Age, atrial fibrillation, and structural heart disease are the main determinants of left atrial fibrosis detected by delayedenhanced magnetic resonance imaging in a general cardiology population. Journal of Cardiovascular Electrophysiology 2015;26:484-492.

19. Mahajan R, Lau DH, Brooks AG, et al. Electrophysiological, Electroanatomical, and Structural Remodeling of the Atria as Consequences of Sustained Obesity. Journal of the American College of Cardiology 2015;66:1-11.

20. Marcus GM, Yang Y, Varosy PD, Ordovas K, Tseng ZH, Badhwar N, Lee BK, Lee $\mathrm{RJ}$, Scheinman MM, Olgin JE. Regional left atrial voltage in patients with atrial fibrillation. Heart Rhythm 2007;4:138-144.

21. Gerstenfeld EP, SippensGroenewegen A, Lux RL, Lesh MD. Derivation of an optimal lead set for measuring ectopic atrial activation from the pulmonary veins by using body surface mapping. Journal of Electrocardiology 2000;33 Suppl:179-185.

22. Ihara Z, van Oosterom A, Jacquemet V, Hoekema R. Adaptation of the standard 12-lead electrocardiogram system dedicated to the analysis of atrial fibrillation. Journal of Electrocardiology 2007;40:68 e61-68.

23. Platonov PG, Carlson J, Ingemansson MP, Roijer A, Hansson A, Chireikin LV, Olsson SB. Detection of inter-atrial conduction defects with unfiltered signalaveraged P-wave ECG in patients with lone atrial fibrillation. Europace 2000;2:32-41. 


\section{Chapter 2}

24. Nielsen JB, Kuhl JT, Pietersen A, et al. P-wave duration and the risk of atrial fibrillation: Results from the Copenhagen ECG Study. Heart Rhythm 2015;12:1887-95.

25. Cheng S, Keyes MJ, Larson MG, McCabe EL, Newton-Cheh C, Levy D, Benjamin EJ, Vasan RS, Wang TJ. Long-term outcomes in individuals with prolonged PR interval or first-degree atrioventricular block. JAMA 2009;301:2571-2577.

26. Platonov PG. P-wave morphology: underlying mechanisms and clinical implications. Annals of Noninvasive Electrocardiology 2012;17:161-169.

27. Kamel H, Soliman EZ, Heckbert SR, Kronmal RA, Longstreth WT, Jr., Nazarian S, Okin PM. P-wave morphology and the risk of incident ischemic stroke in the Multi-Ethnic Study of Atherosclerosis. Stroke 2014;45:2786-2788.

28. Soliman EZ, Prineas RJ, Case LD, Zhang ZM, Goff DC, Jr. Ethnic distribution of ECG predictors of atrial fibrillation and its impact on understanding the ethnic distribution of ischemic stroke in the Atherosclerosis Risk in Communities (ARIC) study. Stroke 2009;40:1204-1211.

29. Holmqvist F, Platonov PG, Carlson J, Zareba W, Moss AJ. Altered interatrial conduction detected in MADIT II patients bound to develop atrial fibrillation. Annals of Noninvasive Electrocardiology 2009;14:268-275. 



\title{
Part II
}

\author{
The ECG \\ predicting \\ rhythm control \\ strategies.
}




\section{Chapter 3}

The ECG as a tool to determine atrial fibrillation complexity

Theo Lankveld, Stef Zeemering, Harry J. Crijns and Ulrich Schotten

Heart 2014;14:1077-84 
Chapter 3

\section{Abstract}

The use of the electrocardiogram for atrial fibrillation (AF) in clinical daily practice is still limited to its diagnosis. Recent research shows however that ECG-derived parameters can also be used to assess the spatiotemporal properties of AF. Specifically, the complexity of the f-waves in the ECG reflect the complexity of the fibrillatory conduction during AF and therefore can be used for quantification of the degree of electrophysiological alterations in the atria. This information might be useful for guiding AF therapy and might form the basis for classification of AF. This review focuses on technical and mathematical aspects of ECG-based atrial complexity assessment and its potential ability to guide treatment strategies. 


\section{Introduction}

The ECG is a widely used tool for confirming clinical diagnosis and guiding therapy. For example, the ECG is essential in diagnosing an acute myocardial infarction and guide percutaneous coronary intervention. In case of ventricular tachycardia or ventricular ectopy, the ECG can be used to suggest an exit point in the left or right ventricle. In atrial fibrillation (AF) however, the ECG is mainly used to confirm the diagnosis, to monitor the effect of antiarrhythmic drugs (AADs) and to adjust rate-control. Some studies show that during sinus rhythm (SR) the P-wave duration can to some extent predict AF after coronary bypass surgery or new-onset AF. ${ }^{1,2}$ Also atrial ectopy in the recovery phase after normal exercise testing predicts AF in subjects with left ventricular hypertrophy. ${ }^{3}$

Recent research shows more can be learned from ECG potentials on the body surface, particularly from the ECG recorded during AF. This review will focus on recent advances in $\mathrm{AF}$ detection using new techniques, atrial complexity parameters that can be derived from the body surface ECG during AF and its potential to guide different therapy strategies.

\section{Advances in AF detection}

Traditional methods to diagnose AF such as a standard 12-lead ECG or ambulatory Holter monitoring have a limited sensitivity to detect AF, particularly in patients with low frequencies of AF paroxysms and limited symptoms. In patients with symptoms possibly due to AF or patients at risk for AF and a "virtual" indication for anticoagulation, easy-to-use devices that can be operated in a home environment might result in earlier AF detection and more effective stroke prevention. A recent study used a patient-operated ECG recorder to detect AF in patients without a history of AF but with at least one AF risk factor and found AF in 7 out of the 132 patients. ${ }^{4}$ In a validation study, this ECG recorder had a sensitivity of $99 \%$ and a specificity of $96 \%$ for AF detection. ${ }^{5}$ In an ongoing study the recently validated MyDiagnostick (Tieleman et al. Europace in press), a metal stick recording and analysing an $E C G$, is used to detect AF during influenza vaccination at the general practitioners office among 3000 participants (NCT02006524). The increasing use of smartphones offers another interesting opportunity for early disease detection. A validation study showed the ability of an iPhone to detect AF with a sensitivity of $96 \%$ and a specificity of $98 \%$ using irregularity of fingertip blood pulsations with the camera. ${ }^{6}$ AliveCor, another iPhone application records a single-lead ECG using two electrodes at the back of the iPhone. The validation study of this application showed a high accuracy in diagnosing AF using an 
automated algorithm determining RR-irregularity and P-wave absence. ${ }^{7}$ This application is currently used in a community-based study to identify undiagnosed AF. ${ }^{8}$ Implantable loop recorders have the ability to detect both symptomatic and non-symptomatic AF during a long time period. In the ongoing REVEAL AF study patients with symptoms and/or at risk for having AF receive an insertable cardiac monitor with a dedicated AF detection algorithm. The purpose is to detect AF episodes $\geq 6$ minutes during 18 months follow-up. ${ }^{9}$ Most of the automated AF algorithms used in the previously discussed recorders are based on the irregularity of the RR-intervals. Short-comings of these algorithms are limited specificity in the presence of ectopic beats and limited sensitivity for detection of short AF-episodes. One study examined the three most commonly used algorithms and found a ROC area of $90.7 \%$ in detecting AF episodes of $5 \mathrm{~s}$ which increased to a ROC area of $94.0 \%$ in episodes of 60 s. $^{10}$ It seems reasonable that when automated algorithms are used at least $60 \mathrm{~s}$ are analysed to detect an AF episode.

\section{Electrophysiological atrial substrate complexity}

Structural heart diseases, ageing and also AF itself cause a slow but steady process of atrial remodelling involving changes in ion channel function, cellular hypertrophy, interstitial fibrosis, inflammatory changes, amyloidosis and fatty infiltration of the atrial myocardium. This process leads to progressive electrical uncoupling between muscle bundles, conduction disturbances, enhanced dispersion of refractoriness, and an increase in spontaneous electrical activity all together increasing the propensity of the atria to AF. These positive feedback mechanisms largely explain the tendency of AF to become more persistent over time. The macroscopic electrophysiological consequence of this process is an increase in conduction block during AF. This increase in conduction block events leading to a higher number of coexisting fibrillation waves has been termed increase in AF complexity. ${ }^{11,12}$

Indeed, the increase in electrophysiological complexity of the AF substrate over time can be demonstrated using invasive direct contact mapping. Several groups showed that patients with persistent AF show a higher number and narrower fibrillation waves compared to patients with acute $A F{ }^{11,13}$ The increase in complexity of the AF substrate has also been demonstrated by noninvasive techniques combining atrial anatomical information with electrograms recorded from the body surface. ${ }^{12}$ 


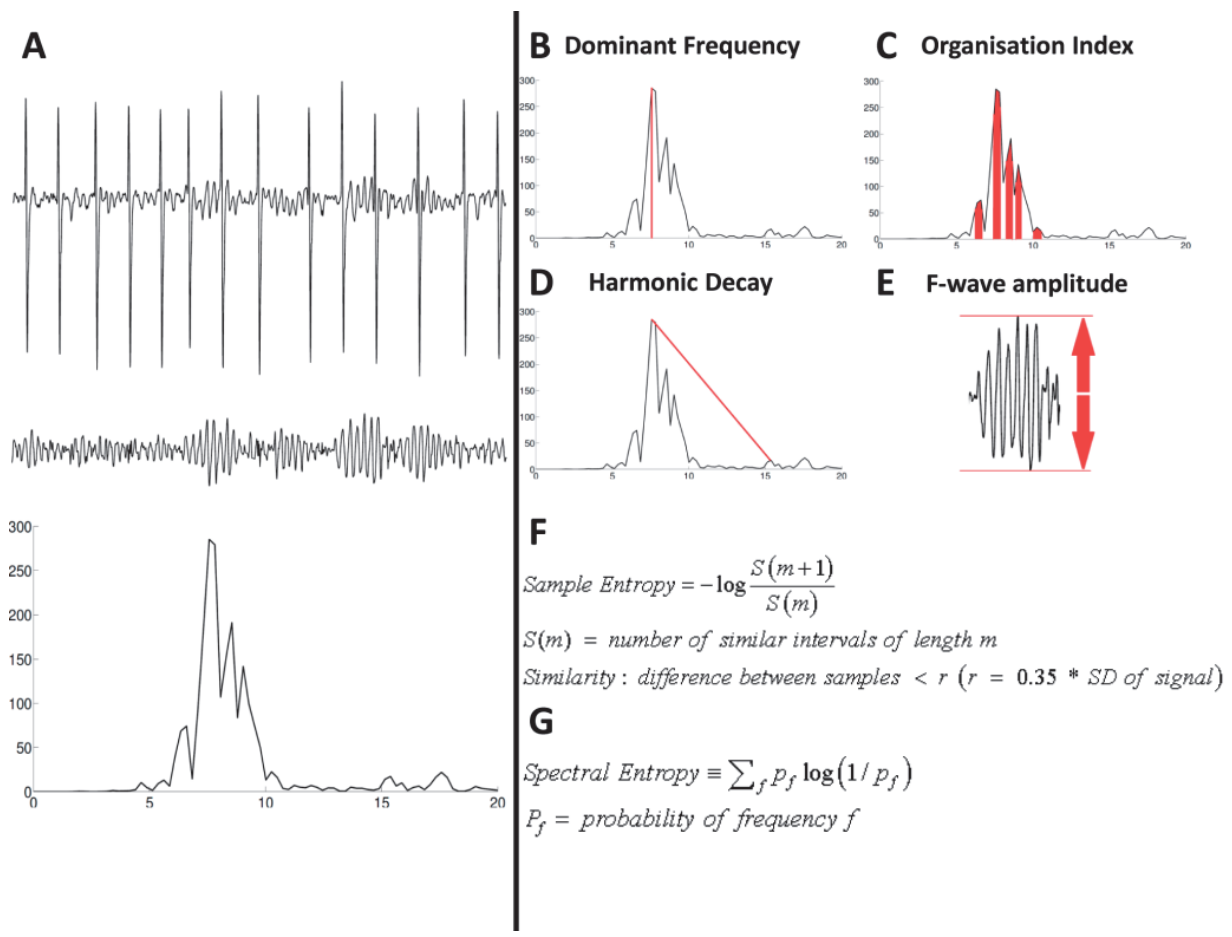

Figure 1: Panel A: Upper panel shows the original ECG signal lead V1, middle panel the atrial signal after QRST cancellation and the bottom left panel shows the frequency spectrum. Right panel shows visualisation of complexity parameters computed on a single lead. A: The red line indicates the highest peak in the power spectrum i.e. the Dominant Frequency. B: The red bars schematically show the areas under the five highest peaks in the power spectrum. The ratio of the total red area dived by the area under the entire power spectrum gives the organisation index. C: The harmonic decay reflects the slope of the line (here indicated in red) connecting the dominant frequency with its first harmonic. D: The F-wave amplitude is the amplitude between the peak and valley of a F-wave. Usually, the mean amplitude during a time-interval is measured. $E$ : Sample entropy examines similarities within time series. Sample entropy is calculated by the negative logarithm of the probability that two sequences which are similar for $m$ points remain similar for $m+1$ points. F: The spectral entropy is the application of Shannon's entropy on a normalised power spectrum. 
Assessment of atrial electrophysiological substrate, apart from just an anatomical approach, has become more important during ablation of (longstanding) persistent AF. ${ }^{14}$ Unfortunately, these mapping procedures can only be applied during an invasive electrophysiological study or open thorax surgery which limits their wide application. In addition, these procedures are costly and time-consuming and need to be validated in more centres. The most important disadvantage relates to the fact that they can only be performed during intervention, while prediction of outcome of therapy is desirable before the intervention. Obviously, compared to an invasive electrophysiological procedure, the surface ECG is much easier to apply. Although it provides a more global presentation of the underlying electrophysiology, AF complexity parameters computed from body surface potentials have been shown to correlate well with invasive electrophysiological complexity. Most research comparing invasive with non-invasive complexity parameters focussed on the dominant atrial frequency (DF). ${ }^{15-19}$ However, also non-invasive sample entropy (SampEn) and F-wave amplitude (FWA) correlate well with complexity parameters applied on invasive recordings. ${ }^{19}$ For example a good correlation was found between the DF on lead V1 and simultaneously measured right atrial frequency. ${ }^{15}$ More recently it has been demonstrated that the left atrial DF is also reflected on the body surface to some extent. ${ }^{17}$ Because of the representation of atrial electrograms on the body surface, a non-invasive approach using the ECG or body surface potential mapping (BSPM) could help to assess a patient's AF substrate complexity in a quick and non-invasive manner and might potentially guide therapy.

\section{ECG guiding rhythm control strategies}

Table 1 and figure 1 give an overview of various AF complexity parameters studied on a standard 12-lead ECG. ${ }^{20}$ These atrial complexity parameters include both time and frequency domain parameters. Some of these investigations have been used to predict spontaneous cardioversion of paroxysmal AF, a lower DF predicts spontaneous cardioversion. ${ }^{21,} 22$ Short AF episodes have a lower DF compared to longer lasting episodes, the DF increases during those episodes and decreases prior to termination. ${ }^{22}$ Most studies, however, focussed on ECG-based prediction of success of a rhythm control strategy in patients with persistent AF. Several non-invasive atrial complexity parameters have a reasonable predictive value for the maintenance of SR after direct current cardioversion (DCC). Sample entropy is lower in patients that maintain SR 4 weeks after DCC. ${ }^{23}$ Furthermore, a higher FWA, a lower harmonic decay and a lower DF predict maintenance of SR. ${ }^{23-26}$ Other studies evaluated prediction of the response to AADs. Patients that 
responded to ibutilide infusion had a significantly lower DF than those who did not. ${ }^{27}$ In another study, a lower DF appeared predictive for the response to oral flecainide. ${ }^{28}$ Furthermore, surface ECG AF complexity parameters have been used to predict catheter ablation outcome in patients with (longstanding) persistent AF. For example, a higher FWA predicted favourable outcome during the ablation, ${ }^{29}$ and at long-term follow-up. ${ }^{30}$ Another study showed that a longer AF cycle length on lead V1 was associated with favourable acute and long-term outcome after catheter ablation. ${ }^{16}$ Although these results seem promising, some studies using DF or SampleEn showed a lower predictive value for outcome after DCC for persistent AF. ${ }^{23,31}$ In another study low DF correlated with successful DCC but not with recurrence of AF during 6 months follow-up. ${ }^{32}$ Possible explanations for these conflicting results might be the use of different preprocessing steps, the use of QRST cancellation as opposed to processing the TQ-interval only, or the different techniques to determine AF complexity. In addition, AAD use differed among studies and $A A D s$ are known to lower the DF. ${ }^{33-35}$ On the other hand, with increasing duration of the AF episode AF complexity increases. ${ }^{34}$ Also, the underlying structural heart disease might have an effect in itself on the AF ECG characteristics. The latter point appears to be particularly important. A lower DF usually means favourable outcome regarding rhythm control therapy. However, in a study on patients with mild to moderate heart failure (HF) a lower DF was associated with increased mortality, mainly due to death from progressing $\mathrm{HF}^{35}$ In patients in whom the AF cycle length was measured invasively during catheter ablation structural heart disease was associated with a low DF. ${ }^{34}$ In an animal study, AF was slower and more organized (i.e lower $\mathrm{DF}$, higher $\mathrm{OI}$ and lower SampEn) in pacing-induced HF as compared to AF induced by vagal stimulation. ${ }^{36}$ Furthermore, an increased amount of fibrosis correlated with a higher $\mathrm{Ol}$ and lower DF. ${ }^{36}$ Another recent study using delayed-enhancement on MRI to quantify atrial fibrosis in patients with (longstanding) persistent AF undergoing catheter ablation also found slower and more organized atrial electrograms at the site of atrial fibrosis. ${ }^{37}$ However, the baseline cycle length measured in the left atrial appendage was shorter in patients with more pronounced fibrosis. Because the ECG provides a more global representation of the electrical complexity the exact effect of atrial fibrosis on the surface ECG still needs to be thoroughly investigated. Also ageing by itself has an effect on atrial complexity parameters. ${ }^{32,35,38,39}$ All these studies found a lower DF in older patients. These changes might be due to increased amount of fibrosis with increasing age.

Additionally, most studies conducted on this topic were relatively small and there is a need to validate the results in larger uniform patient cohorts with, if 
Chapter 3

possible, well characterized underlying pathology. An overview of research already conducted on the prediction of rhythm control therapy using a $10 \mathrm{~s} 12$ lead ECG is provided in Table 2. 
ECG to determine AF complexity

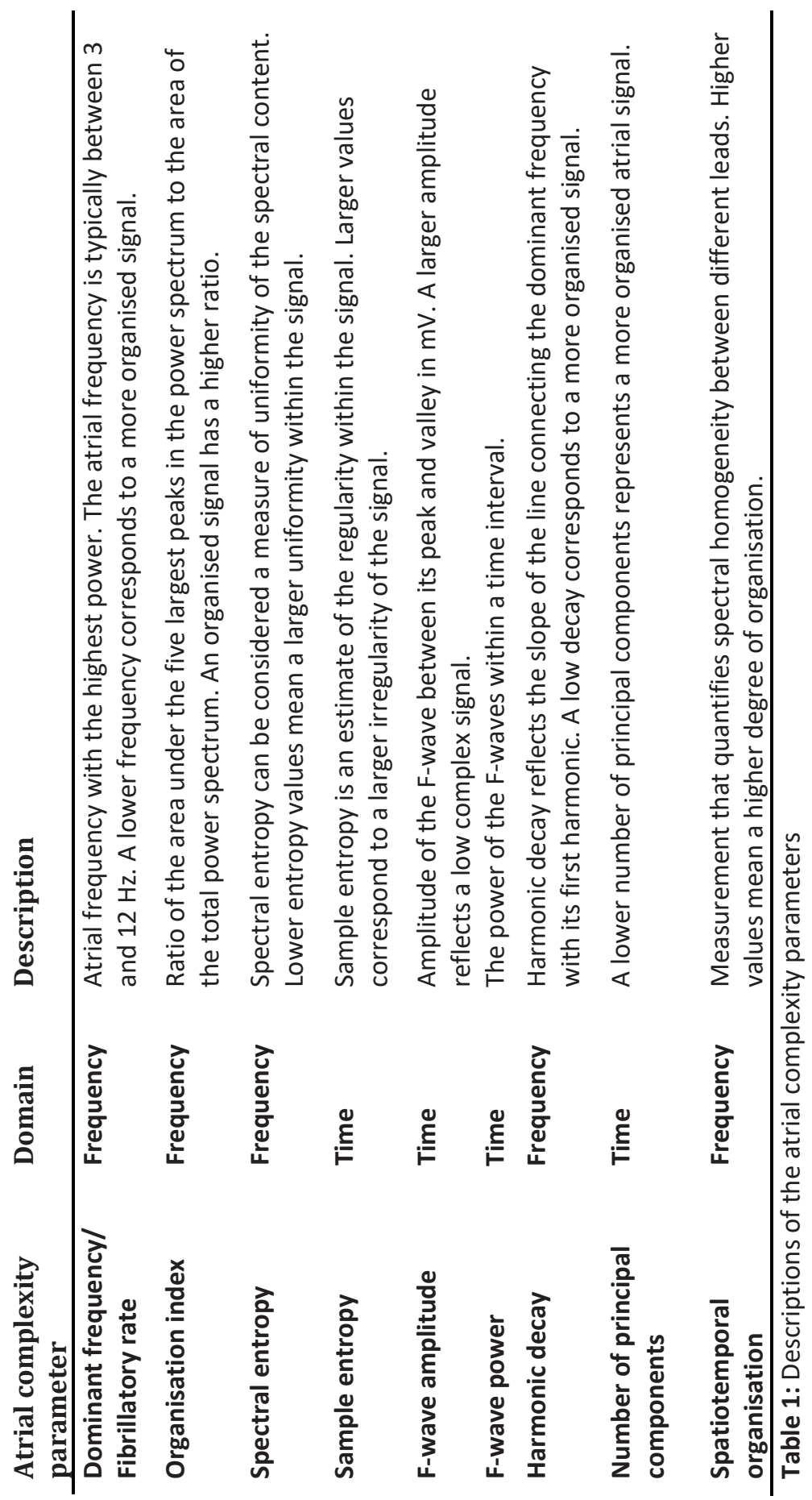


Chapter 3

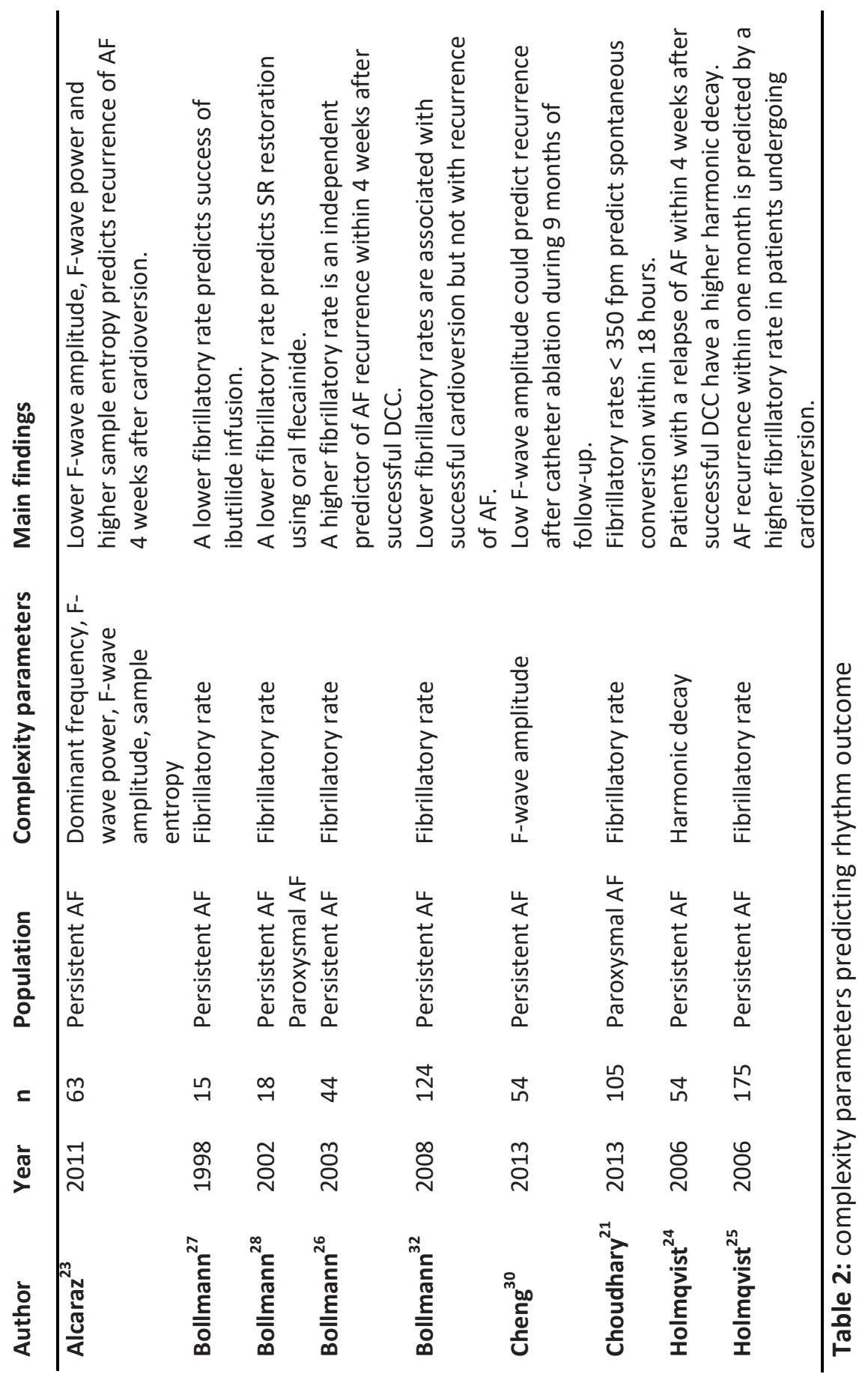


ECG to determine AF complexity

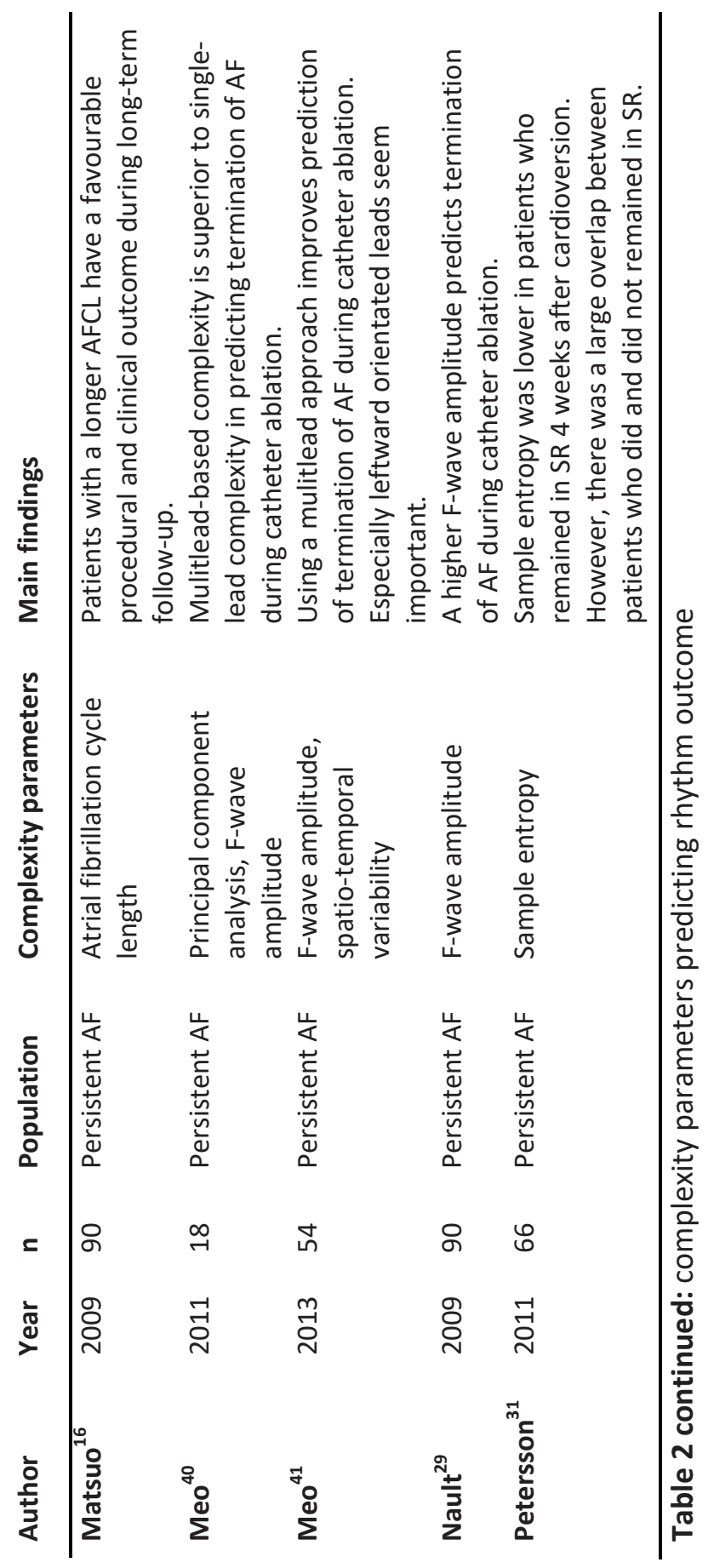




\section{Lead placement}

Most of the above mentioned research focussed on lead V1 because of the high amplitude of the atrial signal compared to other leads (see figure 2). Lead V1 is known to reflect right atrial electrical activity more than left atrial activity. ${ }^{42}$ Recent research, however, showed that the leads positioned more leftward on the thorax (V3-V6) have a higher predictive value for maintenance of SR after ablation than lead V1. ${ }^{41}$ This is remarkable because of the smaller FWA resulting in a smaller signal-to-noise ratio. Obviously, information on left atrial electrical activity is particularly important for prediction of AF recurrences after AF ablation.

In the conventional 12-lead ECG the precordial leads are positioned to follow the global depolarisation and repolarisation of the ventricles. In contrast, to assess (left) atrial signals at the body surface, an alternative lead placement may appear more suitable. One research group developed a lead configuration based on the independent information derived from leads of both simulated and measured BSPMs. ${ }^{43}$ Important to note is the use of one lead on the patient's back which may provide additional electrical information from the left atrium. ${ }^{17}$ Another group also looked at an alternative lead placement with electrodes placed on both the right anterior and posterior side of the thorax. ${ }^{44}$ They showed gradients in fibrillatory rates from anterior to posterior in patients with persistent AF. ECG signals from the left atrium can be detected on the posterior or left anterior side of the thorax, ${ }^{17}$ but also in the oesophagus. $^{45}$ Future research should address the validity of (left) atrial complexity assessment using conventional leads other than lead V1 in monitoring rhythm control strategies and predicting rhythm control outcomes. In addition, alternative lead placements, either at the anterior or posterior chest or in the oesophagus should be evaluated and compared with the conventional ECG. 


\section{Anterior \\ $\mathbf{R}$}

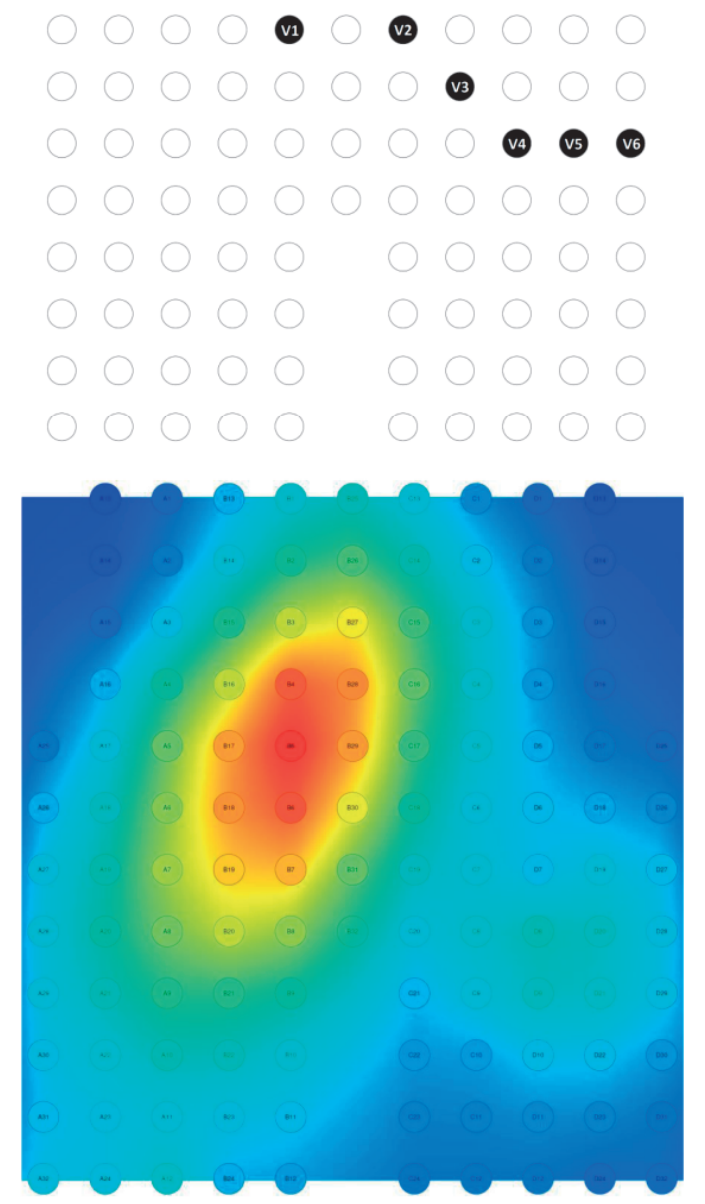

(7)

(8) $จ$

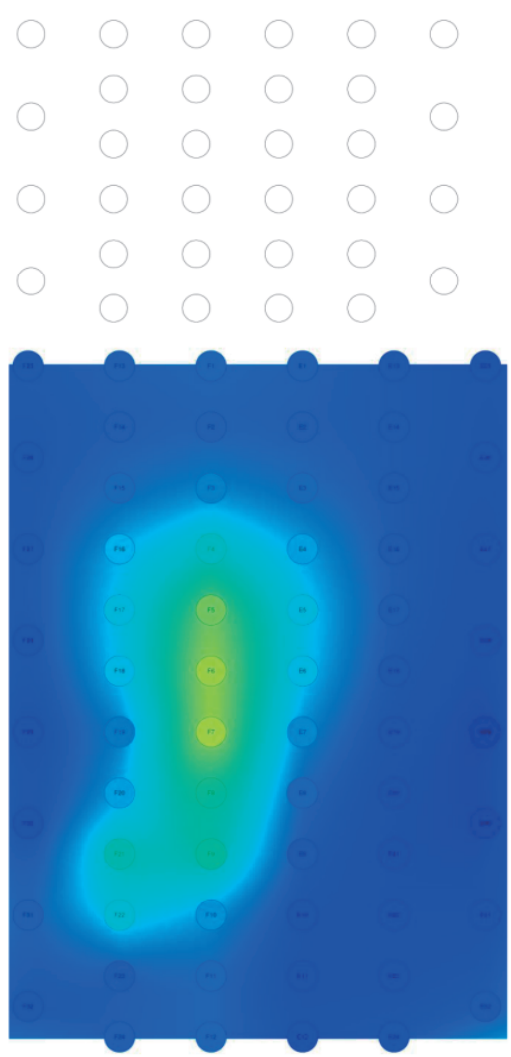

Figure 2: Upper panel shows the distribution of the body surface potential electrodes on the anterior (left) and posterior (right) thorax. The standard 12 lead ECG and additional leads V7-V9 are given as a reference. The lower panel shows the F-wave amplitude of all the electrodes, red means a high and blue a low F-wave amplitude. Shown is the higher F-wave amplitude on the electrodes positioned near lead V1 compared to leads positioned on other parts of the thorax. Remarkable are the little higher amplitudes near lead V9. 


\section{ECG-Imaging guiding ablation strategies}

Another potentially useful application of surface electrocardiography is to guide AF ablation. The cornerstone of catheter ablation for patients with paroxysmal AF is isolating the pulmonary veins. In patients with persistent AF different ablation strategies and methods have been recommended with varying results. In these patients, non-invasive ECG analysis could be useful to determine whether advanced ablation strategies should be applied. In 2004 the first human applications of ECG imaging (ECGI) was reported. ${ }^{46}$ In ECGI, the spatial ECG information obtained from a BSPM is combined with anatomic information of the atria using CT. First, the epicardial propagation of the atrial activation during SR and a typical counterclockwise atrial flutter. In 2010 the same group reported the first data of patients with AF using ECGI. ${ }^{12}$ They showed more pronounced AF complexity in patients with long-standing persistent AF compared to persistent or paroxysmal AF. Furthermore, they suggested that ECGI can distinguish various pathophysiological arrhythmogenic mechanisms, mostly in patients with persistent AF. They found simultaneous wavelets in $92 \%$ of the patients, a single-wave macro re-entry in $8 \%$, rotors in $15 \%$ and focal activity from the pulmonary veins in $69 \%$ and non-pulmonary vein regions in $62 \%$ of cases. Identifying different $A F$ mechanisms in different patients could lead to a patient tailored ablation strategy. A feasibility study in two patients based on this principle showed that ablating active sources identified by ECGI terminated AF during catheter ablation. ${ }^{47}$ Further research in a larger patient cohort with follow-up is needed to explore the potential of this approach.

The clinical application of ECGI is limited by its need for image modalities such as CT or MRI. The first study to use BSPMs during AF did not use an image modality. Nevertheless, the authors were able to demonstrate large variability in the activation patterns between individual patients. ${ }^{48} \mathrm{~A}$ limitation of this study was that no correlation with invasive measurements was investigated. More recent studies investigated the ability of BSPM to identify invasively measured high-frequency sources non-invasively during AF without an image modality. ${ }^{17}$ High-frequency sources are reflected on the surface electrode closest to the atrium of interest: right atrial signals are projected on the rightanterior part of the body surface. Left atrial signals are projected mainly on the posterior and the left-anterior part. ${ }^{17}$ Earlier research showed that the use of BSPMs may help to identify the location of focal triggers for AF using premature atrial activations during SR. ${ }^{49}$ Whether identifying high-frequency sources or focal triggers before arrival at the electrophysiology laboratory can 
guide ablation and improve long-term success certainly warrants further investigation.

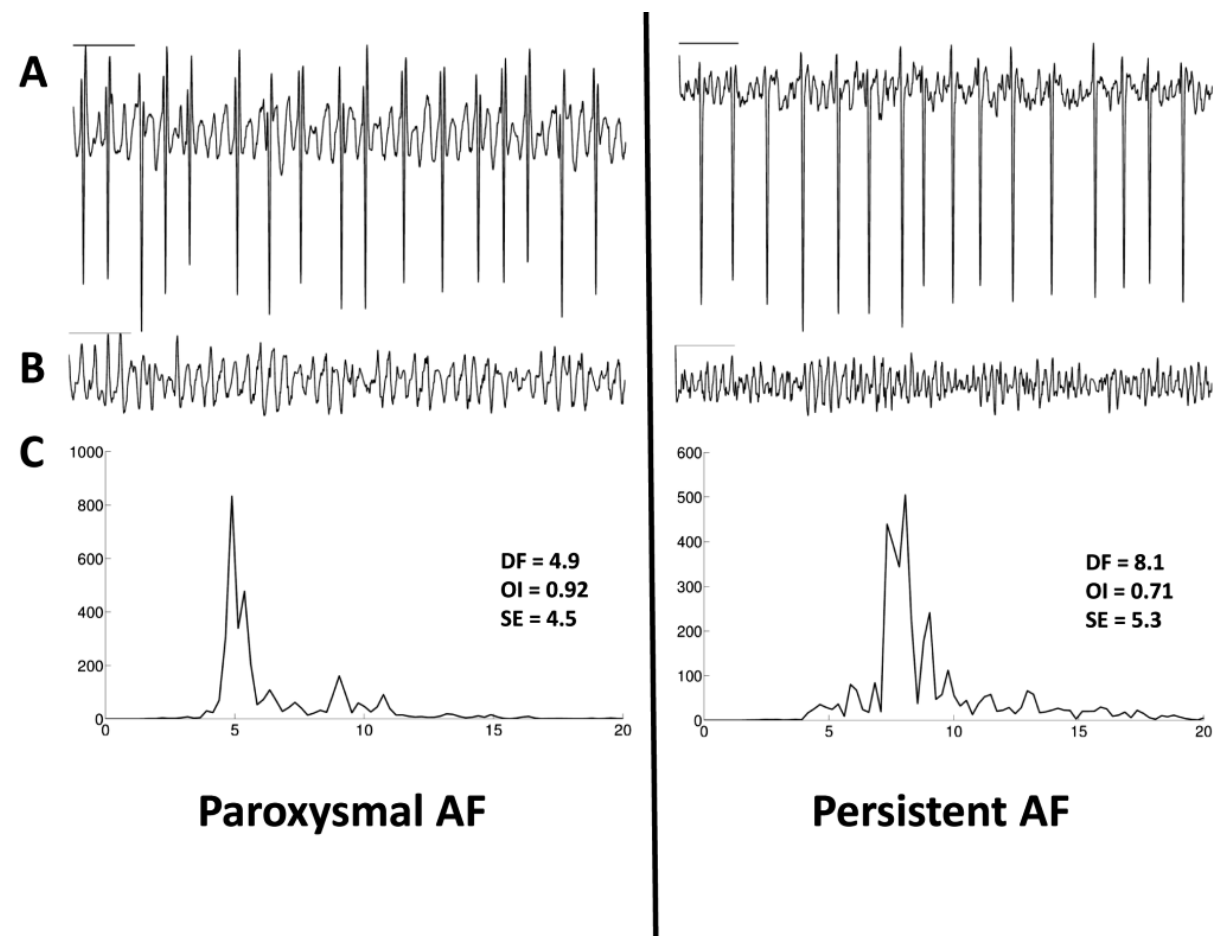

Figure 3: The left panel shows lead V1 from a patient with paroxysmal atrial fibrillation (AF) and the right panel shows lead V1 from a patient with persistent AF. Panel A shows lead V1, panel B shows the atrial signal after QRST cancellation and panel $C$ shows the corresponding frequency spectrum. Shown is a lower dominant frequency (DF) and spectral entropy (SE) and higher organisation index $(\mathrm{OI})$ in the patient with paroxysmal AF compared to the patient with persistent AF. 


\section{ECG based classification}

The ultimate goal of research on AF complexity measures based on the ECG is to develop an AF classification allowing patient-tailored AF treatment as advocated by recent guidelines and consensus conferences. ${ }^{50,51}$ The current classification of $A F$, divided in paroxysmal, persistent or long-standing persistent AF, is purely based on the duration of the AF episodes and whether or not AF terminates by itself. Assessing the duration of AF can be challenging due to high variability of symptoms. The ECG derived atrial complexity parameters mentioned in this review might serve as basis to determine the relative degree of electrophysiological changes occurring over time, because some of these parameters are able to distinguish between patients from the different groups. ${ }^{38,52,53}$ Patients with paroxysmal AF have a lower DF and lower SampEn than patients with persistent AF. ${ }^{38,52}$ Patients with persistent AF have a higher degree of spatiotemporal organisation compared to patients with long-standing persistent $A F{ }^{53}$ Figure 3 shows an example of $V 1$ and the frequency spectrum from a patient with paroxysmal and a patient with persistent AF. The patient with paroxysmal AF shows a lower DF, higher OI and lower SE than the patient with persistent AF. In figure 4 an example of a BSPM from a patient with paroxysmal and persistent AF is given. While the DF is higher in the persistent AF patient (upper panel) the organisation is lower. The degree of these changes certainly partly reflects the duration of AF episodes but also might reflect the presence of predisposing structural alterations due to HF or ageing. These structural alterations - besides duration of AF - also have been shown to be significant determinants of successful rhythm control therapy. ${ }^{54}$ For this reason, ECG-based atrial substrate complexity parameters might be more accurate to classify patients than the clinical history. Figure 5 shows a possible flow-chart for the management of AF patients based on a combination of the patient's symptoms with an ECG-based classification of AF. In patients with a low AF complexity, a rhythm control strategy using either AADs or catheter ablation with pulmonary vein isolation only could be the preferred treatment option. In patients with a highly complex AF substrate rate control might be the best treatment option. Should rhythm control still be pursued, catheter ablation with extensive substrate modification rather than cardioversion and prophylactic AADs could be the most successful treatment. To assess the true value of a model containing different atrial complexity parameters to guide treatment a prospective clinical trial should be undertaken using the ECG as a guide for treatment. 


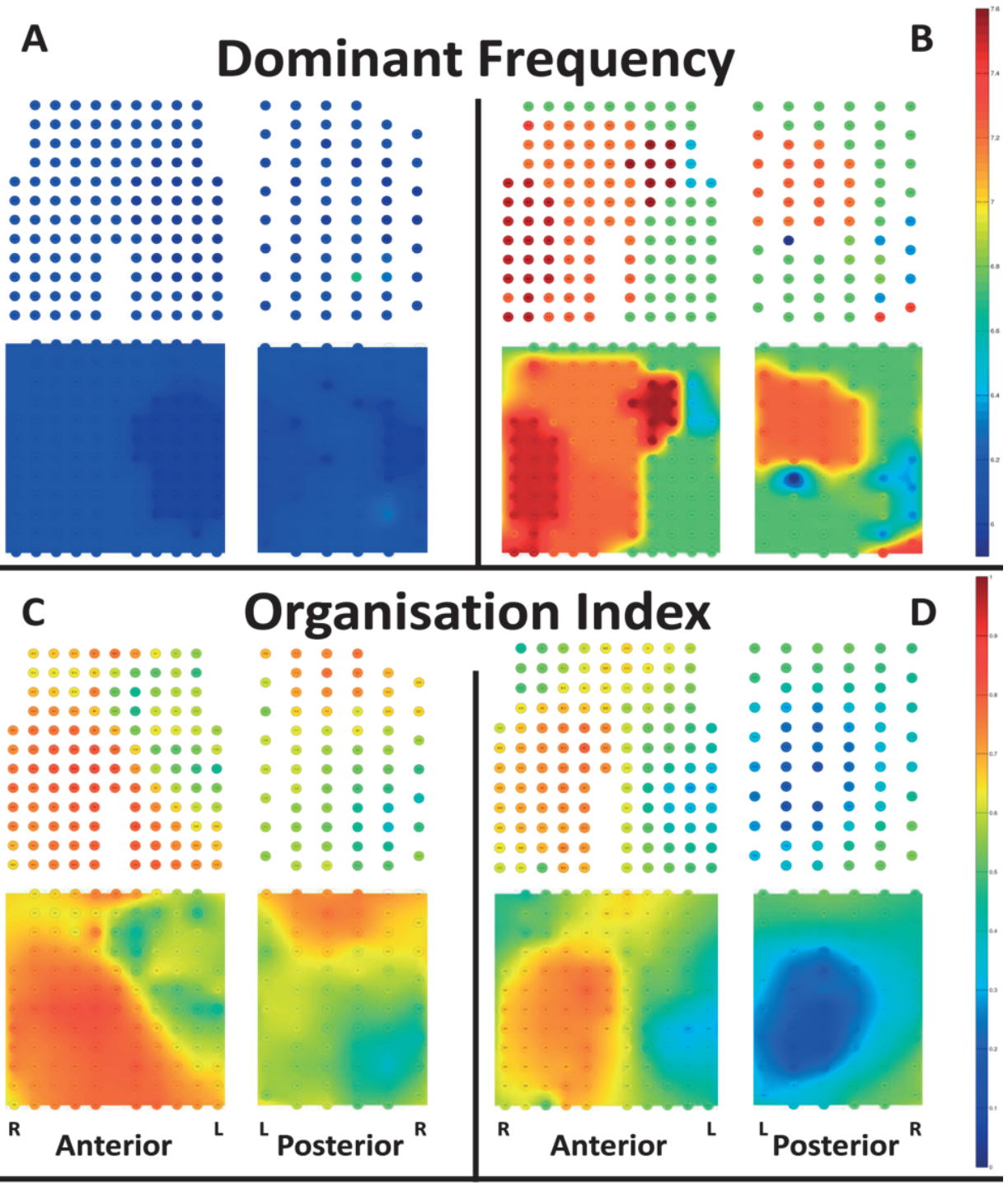

Paroxysmal AF

Persistent AF

Figure 4: Panel A shows the dominant frequency on a body surface potential map of a patient in paroxysmal atrial fibrillation (AF) with first the dominant frequency of every single electrode and below the interpolated signals. Panel B shows the dominant frequency of a patient in persistent $A F$. Panel $C$ and $D$ show the organisation index of the same patients. Blue means a low value were the red colour means a higher value. The dominant frequency in the patient with paroxysmal AF is lower and more evenly distributed on the body surface compared to the patient with persistent AF. Furthermore, the organisation index is higher in the patient with paroxysmal AF. 
Chapter 3

\section{Conclusions}

Atrial complexity parameters derived from a standard 12-lead ECG may be used to identify patients likely to respond to rhythm control therapy, at least during short-term follow-up. There is still only limited evidence for the predictive value for long-term outcome. BSPMs with or without the use of anatomic imaging techniques seem promising in guiding catheter ablation. Expanding the spatial information of a standard 12-lead ECG by applying different or additional lead positioning may help to improve assessment of the (left) atrial electrical signals, which may thereby improve the predictive value of non-invasive atrial AF substrate complexity parameters. The ultimate goal would be to guide AF therapy based on the complexity of the AF substrate of the individual patient.

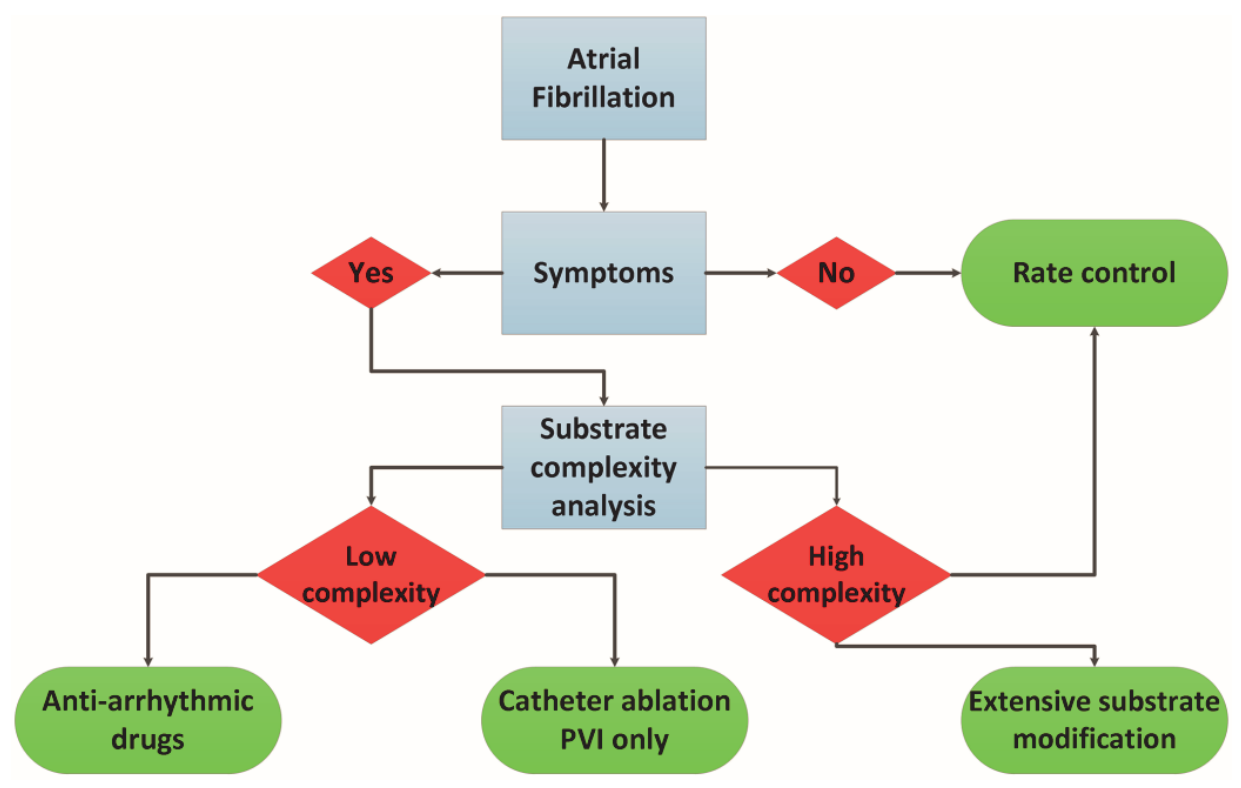

Figure 5: Possible flow-chart for the management of patients with atrial fibrillation (AF) based on a combination of the patient's symptoms with an ECG-based classification of AF. 


\section{References}

1. Magnani JW, Johnson VM, Sullivan LM, Gorodeski EZ, Schnabel RB, Lubitz SA, Levy D, Ellinor PT, Benjamin EJ. P wave duration and risk of longitudinal atrial fibrillation in persons $>/=60$ years old (from the Framingham Heart Study). The American Journal of Cardiology 2011;107:917-921 e911.

2. Zhang BC, Che WL, Li WM, Xu YW. Meta-analysis of $P$ wave character as predictor of atrial fibrillation after coronary artery bypass grafting. International Journal of Cardiology 2011;152:260-262.

3. Folkeringa RJ, Hartgers J, Tieleman RG, Gorgels AP, Dassen WR, Crijns HJ. Atrial extrasystoles after exercise predict atrial fibrillation in patients with left ventricular hypertrophy. Heart 2006;92:545-546.

4. Samol A, Masin M, Gellner R, Otte B, Pavenstadt HJ, Ringelstein EB, Reinecke $\mathrm{H}$, Waltenberger J, Kirchhof $\mathrm{P}$. Prevalence of unknown atrial fibrillation in patients with risk factors. Europace 2013;15:657-662.

5. Kaleschke G, Hoffmann B, Drewitz I, Steinbeck G, Naebauer M, Goette A, Breithardt G, Kirchhof P. Prospective, multicentre validation of a simple, patient-operated electrocardiographic system for the detection of arrhythmias and electrocardiographic changes. Europace 2009;11:1362-1368.

6. McManus DD, Lee J, Maitas O, Esa N, Pidikiti R, Carlucci A, Harrington J, Mick $\mathrm{E}$, Chon $\mathrm{KH}$. A novel application for the detection of an irregular pulse using an iPhone 4S in patients with atrial fibrillation. Heart rhythm 2013;10:315-319.

7. Lau JK, Lowres N, Neubeck L, Brieger DB, Sy RW, Galloway CD, Albert DE, Freedman SB. iPhone ECG application for community screening to detect silent atrial fibrillation: a novel technology to prevent stroke. International Journal of Cardiology 2013;165:193-194.

8. Lowres N, Freedman SB, Redfern J, McLachlan A, Krass I, Bennett A, Briffa T, Bauman A, Neubeck L. Screening Education And Recognition in Community pHarmacies of Atrial Fibrillation to prevent stroke in an ambulant population aged >=65 years (SEARCH-AF stroke prevention study): a cross-sectional study protocol. BMJ open 2012;2.

9. Reiffel J, Verma A, Halperin JL, Gersh B, Tombul S, Carrithers J, Sherfesee L, Kowey P. Rationale and design of REVEAL AF: a prospective study of previously undiagnosed atrial fibrillation as documented by an insertable cardiac monitor in high-risk patients. American Heart Journal 2014;167:22-27.

10. Langley P, Dewhurst M, Di Marco LY, Adams P, Dewhurst F, Mwita JC, Walker $R$, Murray A. Accuracy of algorithms for detection of atrial fibrillation from short duration beat interval recordings. Medical Engineering \& Physics 2012;34:1441-1447.

11. Allessie MA, de Groot NM, Houben RP, Schotten U, Boersma E, Smeets JL, Crijns HJ. Electropathological substrate of long-standing persistent atrial fibrillation in patients with structural heart disease: longitudinal dissociation. Circulation Arrhythmia and Electrophysiology 2010;3:606-615. 


\section{Chapter 3}

12. Cuculich PS, Wang Y, Lindsay BD, Faddis MN, Schuessler RB, Damiano RJ, Jr., Li L, Rudy Y. Noninvasive characterization of epicardial activation in humans with diverse atrial fibrillation patterns. Circulation 2010;122:1364-1372.

13. Lee $\mathrm{G}$, Kumar $\mathrm{S}$, Teh $\mathrm{A}$, et al. Epicardial wave mapping in human long-lasting persistent atrial fibrillation: transient rotational circuits, complex wavefronts, and disorganized activity. European Heart Journal 2014;35:86-97.

14. Narayan SM, Krummen DE, Shivkumar K, Clopton P, Rappel WJ, Miller JM. Treatment of atrial fibrillation by the ablation of localized sources: CONFIRM (Conventional Ablation for Atrial Fibrillation With or Without Focal Impulse and Rotor Modulation) trial. Journal of the American College of Cardiology 2012;60:628-636.

15. Holm M, Pehrson S, Ingemansson M, Sornmo L, Johansson R, Sandhall $L$, Sunemark M, Smideberg B, Olsson C, Olsson SB. Non-invasive assessment of the atrial cycle length during atrial fibrillation in man: introducing, validating and illustrating a new ECG method. Cardiovascular Research 1998;38:69-81.

16. Matsuo S, Lellouche $N$, Wright $M$, et al. Clinical predictors of termination and clinical outcome of catheter ablation for persistent atrial fibrillation. Journal of the American College of Cardiology2009;54:788-795.

17. Guillem MS, Climent AM, Millet J, Arenal A, Fernandez-Aviles F, Jalife J, Atienza F, Berenfeld O. Noninvasive localization of maximal frequency sites of atrial fibrillation by body surface potential mapping. Circulation Arrhythmia and Electrophysiology 2013;6:294-301.

18. Roithinger FX, SippensGroenewegen A, Karch MR, Steiner PR, Ellis WS, Lesh MD. Organized activation during atrial fibrillation in man: endocardial and electrocardiographic manifestations. Journal of Cardiovascular Electrophysiology 1998;9:451-461.

19. Alcaraz R, Hornero F, Rieta JJ. Assessment of non-invasive time and frequency atrial fibrillation organization markers with unipolar atrial electrograms. Physiological Measurement 2011;32:99-114.

20. Schotten $U$, Maesen B, Zeemering S. The need for standardization of timeand frequency-domain analysis of body surface electrocardiograms for assessment of the atrial fibrillation substrate. Europace 2012;14:1072-1075.

21. Choudhary MB, Holmqvist F, Carlson J, Nilsson HJ, Roijer A, Platonov PG. Low atrial fibrillatory rate is associated with spontaneous conversion of recentonset atrial fibrillation. Europace 2013;15:1445-1452.

22. Bollmann A, Sonne K, Esperer HD, Toepffer I, Langberg JJ, Klein HU. Noninvasive assessment of fibrillatory activity in patients with paroxysmal and persistent atrial fibrillation using the Holter ECG. Cardiovascular Research 1999;44:60-66.

23. Alcaraz R, Hornero F, Rieta JJ. Noninvasive time and frequency predictors of long-standing atrial fibrillation early recurrence after electrical cardioversion. PACE 2011;34:1241-1250.

24. Holmqvist F, Stridh M, Waktare JE, Roijer A, Sornmo L, Platonov PG, Meurling $\mathrm{CJ}$. Atrial fibrillation signal organization predicts sinus rhythm maintenance in 
patients undergoing cardioversion of atrial fibrillation. Europace 2006;8:559565.

25. Holmqvist F, Stridh M, Waktare JE, Sornmo L, Olsson SB, Meurling CJ. Atrial fibrillatory rate and sinus rhythm maintenance in patients undergoing cardioversion of persistent atrial fibrillation. European Heart Journal 2006;27:2201-2207.

26. Bollmann A, Husser D, Steinert R, Stridh M, Soernmo L, Olsson SB, Polywka D, Molling J, Geller C, Klein HU. Echocardiographic and Electrocardiographic Predictors for Atrial Fibrillation Recurrence Following Cardioversion. Journal of Cardiovascular Electrophysiology 2003;14:S162-S165.

27. Bollmann A, Kanuru NK, McTeague KK, Walter PF, DeLurgio DB, Langberg JJ. Frequency analysis of human atrial fibrillation using the surface electrocardiogram and its response to ibutilide. The American Journal of Cardiology 1998;81:1439-1445.

28. Bollmann A, Binias KH, Toepffer I, Molling J, Geller C, Klein HU. Importance of left atrial diameter and atrial fibrillatory frequency for conversion of persistent atrial fibrillation with oral flecainide. The American Journal of Cardiology 2002;90:1011-1014.

29. Nault I, Lellouche N, Matsuo S, et al. Clinical value of fibrillatory wave amplitude on surface ECG in patients with persistent atrial fibrillation. Journal of Interventional Cardiac Electrophysiology 2009;26:11-19.

30. Cheng Z, Deng H, Cheng K, Chen T, Gao P, Yu M, Fang Q. The amplitude of fibrillatory waves on leads aVF and V1 predicting the recurrence of persistent atrial fibrillation patients who underwent catheter ablation. Annals of Noninvasive Electrocardiology Jul 2013;18:352-358.

31. Petersson R, Sandberg F, Platonov PG, Holmqvist F. Noninvasive estimation of organization in atrial fibrillation as a predictor of sinus rhythm maintenance. Journal of Electrocardiology 2011;44:171-175.

32. Bollmann A, Tveit A, Husser D, Stridh M, Sornmo L, Smith P, Olsson SB. Fibrillatory rate response to candesartan in persistent atrial fibrillation. Europace 2008;10:1138-1144.

33. Husser D, Stridh M, Sornmo L, Geller C, Klein HU, Olsson SB, Bollmann A. Time-frequency analysis of the surface electrocardiogram for monitoring antiarrhythmic drug effects in atrial fibrillation. The American Journal of Cardiology 2005;95:526-528.

34. Haissaguerre M, Lim KT, Jacquemet V, Rotter M, Dang L, Hocini M, Matsuo S, Knecht $S$, Jais $P$, Virag N. Atrial fibrillatory cycle length: computer simulation and potential clinical importance. Europace 2007;9 Suppl 6:vi64-70.

35. Platonov PG, Cygankiewicz I, Stridh M, Holmqvist F, Vazquez R, Bayes-Genis A, McNitt $S$, Zareba $W$, de Luna $A B$. Low atrial fibrillatory rate is associated with poor outcome in patients with mild to moderate heart failure. Circulation Arrhythmia and Electrophysiology 2012;5:77-83.

36. Koduri H, Ng J, Cokic I, Aistrup GL, Gordon D, Wasserstrom JA, Kadish AH, Lee R, Passman R, Knight BP, Goldberger JJ, Arora R. Contribution of fibrosis and 
Chapter 3

the autonomic nervous system to atrial fibrillation electrograms in heart failure. Circulation Arrhythmia and Electrophysiology 2012;5:640-649.

37. Jadidi AS, Cochet $\mathrm{H}$, Shah AJ, et al. Inverse relationship between fractionated electrograms and atrial fibrosis in persistent atrial fibrillation: combined magnetic resonance imaging and high-density mapping. Journal of the American College of Cardiology 2013;62:802-812.

38. Xi Q, Sahakian AV, Frohlich TG, Ng J, Swiryn S. Relationship between pattern of occurrence of atrial fibrillation and surface electrocardiographic fibrillatory wave characteristics. Heart rhythm 2004;1:656-663.

39. Husser D, Cannom DS, Bhandari AK, Stridh M, Sornmo L, Olsson SB, Bollmann A. Electrocardiographic characteristics of fibrillatory waves in new-onset atrial fibrillation. Europace 2007;9:638-642.

40. Meo M, Zarzoso V, Meste O, Latcu DG, Saoudi N. Non-invasive prediction of catheter ablation outcome in persistent atrial fibrillation by exploiting the spatial diversity of surface ECG. Conference proceedings : Annual International Conference of the IEEE Engineering in Medicine and Biology Society IEEE Engineering in Medicine and Biology Society Conference 2011;2011:5531-5534.

41. Meo M, Zarzoso V, Meste O, Latcu DG, Saoudi N. Spatial variability of the 12lead surface ECG as a tool for noninvasive prediction of catheter ablation outcome in persistent atrial fibrillation. IEEE transactions on bio-medical engineering 2013;60:20-27.

42. Bollmann A, Husser D, Mainardi L, Lombardi F, Langley P, Murray A, Rieta JJ, Millet J, Olsson SB, Stridh M, Sornmo L. Analysis of surface electrocardiograms in atrial fibrillation: techniques, research, and clinical applications. Europace 2006;8:911-926.

43. Ihara Z, van Oosterom A, Jacquemet V, Hoekema R. Adaptation of the standard 12-lead electrocardiogram system dedicated to the analysis of atrial fibrillation. Journal of Electrocardiology Jan 2007;40:68 e61-68.

44. Husser D, Stridh M, Sornmo L, Toepffer I, Klein HU, Bertil Olsson S, Bollmann A. Electroatriography - time-frequency analysis of atrial fibrillation from modified 12-lead ECG configurations for improved diagnosis and therapy. Medical Hypotheses 2007;68:568-573.

45. Binkley PF, Bush CA, Fleishman BL, Leier CV. In vivo validation of the origin of the esophageal electrocardiogram. Journal of the American College of Cardiology 1986;7:813-818.

46. Ramanathan C, Ghanem RN, Jia P, Ryu K, Rudy Y. Noninvasive electrocardiographic imaging for cardiac electrophysiology and arrhythmia. Nature Medicine 2004;10:422-428.

47. Haissaguerre $M$, Hocini $M$, Shah AJ, Derval $N$, Sacher $F$, Jais $P$, Dubois $R$. Noninvasive panoramic mapping of human atrial fibrillation mechanisms: a feasibility report. Journal of Cardiovascular Electrophysiology 2013;24:711717. 
48. Guillem MS, Climent AM, Castells F, Husser D, Millet J, Arya A, Piorkowski C, Bollmann A. Noninvasive mapping of human atrial fibrillation. Journal of Cardiovascular Electrophysiology 2009;20:507-513.

49. SippensGroenewegen A, Natale A, Marrouche NF, Bash D, Cheng J. Potential role of body surface ECG mapping for localization of atrial fibrillation trigger sites. Journal of Electrocardiology 2004;37:47-52.

50. Camm AJ, Kirchhof P, Lip GY, et al. Guidelines for the management of atrial fibrillation: the Task Force for the Management of Atrial Fibrillation of the European Society of Cardiology (ESC). European Heart Journal 2010;31:23692429.

51. Kirchhof $P$, Breithardt $G$, Aliot $E$, et al. Personalized management of atrial fibrillation: Proceedings from the fourth Atrial Fibrillation competence NETwork/European Heart Rhythm Association consensus conference. Europace 2013;15:1540-1556.

52. Alcaraz R, Sandberg F, Sornmo L, Rieta JJ. Classification of paroxysmal and persistent atrial fibrillation in ambulatory ECG recordings. IEEE transactions on bio-medical engineering 2011;58:1441-1449.

53. Uldry L, Van Zaen J, Prudat Y, Kappenberger L, Vesin JM. Measures of spatiotemporal organization differentiate persistent from long-standing atrial fibrillation. Europace 2012;14:1125-1131.

54. Camm AJ, Breithardt G, Crijns H, Dorian P, Kowey P, Le Heuzey JY, Merioua I, Pedrazzini L, Prystowsky EN, Schwartz PJ, Torp-Pedersen C, Weintraub W. Real-life observations of clinical outcomes with rhythm- and rate-control therapies for atrial fibrillation RECORDAF (Registry on Cardiac Rhythm Disorders Assessing the Control of Atrial Fibrillation). Journal of the American College of Cardiology 2011;58:493-501. 



\section{Chapter 4}

Systematic comparison of noninvasive assessment of atrial fibrillation complexity to predict outcome of pharmacological cardioversion in patients with atrial fibrillation

Stef Zeemering*, Theo Lankveld*, Pietro Bonizzi, lone Limantoro, Sebastiaan Bekkers, Harry J. Crijns and Ulrich Schotten

*Contributed equally to this work

Submitted 
Chapter 4

\begin{abstract}
Objective Non-invasive characterization of atrial fibrillation (AF) substrate complexity based on the 12-lead ECG may be used to improve outcome prediction in patients receiving rhythm control therapies of AF. Multiple parameters to assess AF complexity and predict treatment outcome have been suggested. A comparative study of the predictive performance of AF complexity parameters on a large patient population is needed to move towards standardization of non-invasive analysis of AF.
\end{abstract}

Methods: Several non-invasive AF complexity parameters were systematically compared in ECGs of patients with recent onset AF undergoing pharmacological cardioversion (PCV) with flecainide. Parameters were computed on 10-second 12-lead ECGs of 221 patients before drug administration. The ability of ECG parameters derived from single and multiple leads to predict successful PCV was evaluated and compared to common clinical predictors.

Results: Prediction performance of one parameter computed on one lead was best using dominant atrial frequency (lead II, cross-validated receiver operating area under curve (AUC) 0.66, 95\% confidence interval [0.64-0.67]). Multidimensional parameters, computing a single value on multiple leads, showed similar performance (AUC 0.64 [0.62-0.66] using temporal spectral variance). Combining different parameters strongly improved performance (AUC 0.78 [0.76-0.79]). Predictive value of clinical parameters was moderate (AUC 0.68 [0.66-0.70], using right atrial volume and weight), and was most improved by adding frequency domain ECG parameters (AUC 0.81 [0.79-0.82], $\mathrm{p}<0.001)$. Interestingly, ECG parameters also predicted progression to persistent AF during follow-up.

Conclusion: Assessment of AF complexity from 12-lead ECGs is superior to common clinical predictors in predicting successful PCV in AF patients. Frequency-domain parameters showed better predictive performance than time-domain parameters. 


\section{Introduction}

Atrial fibrillation (AF) is a common cardiac arrhythmia that progresses in complexity over time, demonstrated both invasively ${ }^{1,2}$ and non-invasively. ${ }^{3} \mathrm{AF}$ is classified as paroxysmal or persistent mainly based on AF episode duration and cardioversion attempts undertaken. ${ }^{4} \mathrm{~A}$ decision on rhythm control strategy is taken based on this classification, the patient's symptoms, and physician's and patient's preference. Whether a patient will respond to rhythm control therapy is difficult to predict. Moreover, any kind of rhythm control strategy is associated with considerable risks, such as ventricular proarrhythmia in case of anti-arrhythmic drugs or procedural risks in case of AF ablation. Predicting acute and long-term success of AF treatment at any stage of the disease is therefore desirable and subject to extensive research. ${ }^{5}$

Progression of AF complexity is marked by an increase in number of fibrillation waves, caused by increasing incidence of conduction block in the atria as a consequence of a progressive structural remodelling. ${ }^{1}$ The standard 12 -lead ECG is an attractive choice for non-invasive assessment of the level of AF complexity because of its widespread use in daily clinical practice. However, whether AF complexity quantified from the surface ECG can be employed in a clinical setting to predict treatment outcome and ultimately guide management of AF, still has to be established. Many complexity parameters derived from the 12-lead ECG have been proposed. ${ }^{5,6}$ Although several studies report encouraging results in either classifying $A F$ or predicting treatment outcome, it is not a straightforward task to compare and interpret these results, because of large differences in patient populations, the parameters computed on the ECG, and the specific clinical setting. There is a clear need for standardization of ECG-based AF complexity analysis to predict response to therapy and develop an individualised treatment strategy. 6

To address these issues, we investigated a large set of ECG-derived AF complexity parameters and their ability to predict successful outcome of pharmacological cardioversion (PCV) using flecainide in a population of patients with recent onset AF. Long-term implications of observed AF complexity were investigated by linking individual patient AF complexity prior to cardioversion to progression to persistent AF. 
Chapter 4

\section{Methods}

\section{Patient database}

Patient data were retrieved from a database at Maastricht University Medical Center, Maastricht, the Netherlands, of consecutive patients with short lasting episodes of $A F(<48 \mathrm{~h}$, recurrent or new onset $A F)$ undergoing cardioversion with the anti-arrhythmic drug flecainide for the first time between the years 2008 and 2012. From the database a total of 221 patients were selected for this study. Exclusion criteria were the use of anti-arrhythmic drugs (AAD) prior to the PCV attempt, use of additional medication during the PCV procedure, and a missing or poor quality ECG (assessed visually). Patient characteristics are listed in Table 1. Echocardiographic parameters were included if echocardiography was recorded within one year before or after the PCV attempt (available in 139 patients). A 10-second 12-lead ECG was recorded during AF for each patient before the PCV attempt, using a GE MAC 5500 resting ECG recording device (sampling frequency $250 \mathrm{~Hz}$ ). PCV success was defined as restoration of sinus rhythm within one hour after starting the flecainide infusion. Flecainide was dosed at $2 \mathrm{mg} / \mathrm{kg}$ with a maximum dose of $150 \mathrm{mg}$ intravenously. Follow-up data on progression to persistent AF (AF episode duration $>7$ days or electrical cardioversion attempt) within the period January 2008-March 2015 was available for 201 patients.

\section{Non-invasive AF complexity parameters}

The list of non-invasive AF complexity parameters included in this study was composed of parameters that appeared frequently in the last decade of noninvasive AF complexity literature. See Supplemental Materials for parameter definitions and interpretation. Parameters are often computed on the extracted atrial activity (AA), but some can also be computed on (concatenated) TQ intervals. Parameters were computed with algorithms provided by the original author(s) or otherwise as described in the original publication. An overview of parameters and their domain is shown in Figure 1.

Before parameter computation, ECGs were filtered with a $1-100 \mathrm{~Hz}$ band-pass filter ( ${ }^{\text {rd }}$ order Chebyshev, 20dB stop-band attenuation). To enable analysis of TQ-segments, the end of the T-wave and onset of the Q-wave were detected in unfiltered signals using Woody's improved method. Ventricular QRST complexes were removed by a single lead cancellation method based on singular value decomposition of QRST windows. The extracted atrial signals were filtered with a $3 \mathrm{~Hz}$ high-pass filter to remove any remaining $\mathrm{T}$-wave 
residues. Finally, the first and last second were truncated to avoid the border effect of filtering procedures, leaving 8 seconds available for analysis.

Table 1: Patient characteristics

\begin{tabular}{|c|c|c|c|}
\hline Characteristic & $\begin{array}{c}\text { Successful PCV } \\
n=157 \text { (71\%) }\end{array}$ & $\begin{array}{c}\text { Unsuccessful PCV } \\
n=64(29 \%)\end{array}$ & $\begin{array}{c}\mathrm{P} \text { - } \\
\text { value }\end{array}$ \\
\hline Male (\%) & $93(59 \%)$ & $52(81 \%)$ & 0.002 \\
\hline Age (years) & $61 \pm 13$ & $57 \pm 15$ & 0.170 \\
\hline Height $(\mathrm{cm})$ & $174 \pm 10(116)$ & $179 \pm 12(51)$ & 0.004 \\
\hline Weight (kg) & $81 \pm 14(116)$ & $91 \pm 19(51)$ & 0.001 \\
\hline BMI $\left(\mathrm{kg} / \mathrm{m}^{2}\right)$ & $26.9 \pm 3.9(116)$ & $28.1 \pm 5.3(51)$ & 0.305 \\
\hline Diabetes & $11(141)$ & $5(61)$ & 0.924 \\
\hline Hypertension & $66(141)$ & $29(62)$ & 0.996 \\
\hline COPD & $8(141)$ & $2(61)$ & 0.471 \\
\hline PVI & $2(141)$ & $5(60)$ & 0.014 \\
\hline LAD (mm) & $40.3 \pm 5.1(113)$ & $43.1 \pm 6.0(49)$ & 0.003 \\
\hline $\operatorname{LAV}(\mathrm{ml})$ & $74.2 \pm 20.8(111)$ & $80.8 \pm 19.6(48)$ & 0.067 \\
\hline $\operatorname{RAV}(\mathrm{ml})$ & $56.3 \pm 18.0(99)$ & $69.9 \pm 23.3(47)$ & $<0.001$ \\
\hline LVEDD (mm) & $49.4 \pm 5.4$ (117) & $51.5 \pm 6.0(51)$ & 0.127 \\
\hline LVESD (mm) & $33.6 \pm 4.5(116)$ & $36.3 \pm 7.3(50)$ & 0.064 \\
\hline LVEF (\%) & $60.1 \pm 5.6(117)$ & $57.0 \pm 10.0(51)$ & 0.171 \\
\hline $\mathrm{CHA}_{2} \mathrm{DS}_{2}$-VASc & $2[0-3](140)$ & $1[0-3](61)$ & 0.159 \\
\hline HATCH & $1[0-1](140)$ & $1[0-1](61)$ & 0.621 \\
\hline
\end{tabular}

COPD: chronic obstructive pulmonary disease; LAD: left atrial diameter; LAV: left atrial volume; RAV: right atrial volume; LVEDD/LVESD: left ventricular end diastolic/systolic diameter; LVEF: left ventricular ejection fraction; PVI: pulmonary vein isolation 


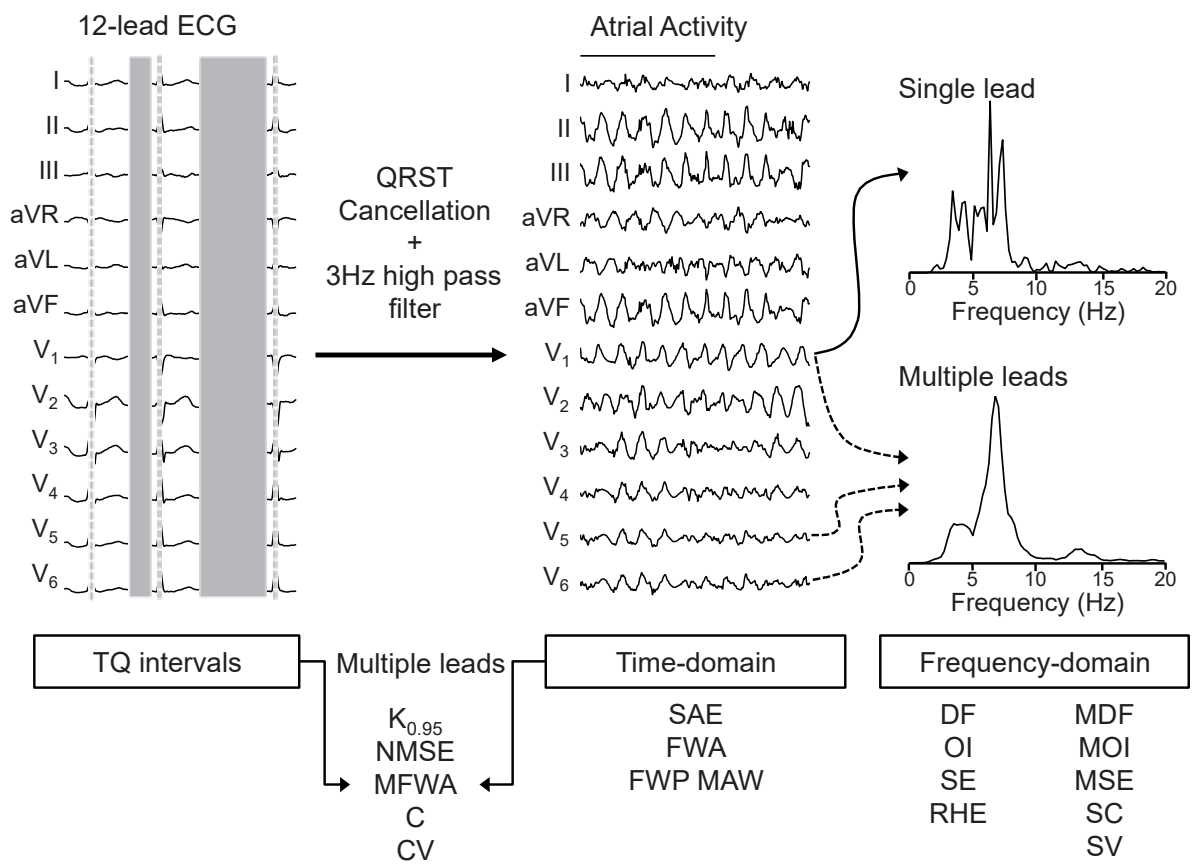

Figure 1: Overview of ECG signal processing and complexity parameter computation. In the timedomain, multidimensional parameters derived from multiple leads can be computed on both the extracted atrial activity, as well as on the TQ-segments of the original ECG. In the frequencydomain, complexity can be quantified based on spectra computed from a single lead or multiple leads. DF: Dominant Frequency, OI: Organization Index, SE: Spectral Entropy, RHE: Relative Harmonic Energy, MDF/MOI/MSE: Multidimensional DF/OI/SE, SC: Spectral Concentration, SV: Spectral Variability, SAE: Sample Entropy, FWA: Fibrillation Wave Amplitude, FWP MAW: Fibrillation Wave Power of the Main Atrial Wave, $\mathrm{K}_{0.95}, \mathrm{C}$ : spatial complexity parameters, NMSE, CV: Variability of spatial complexity, MFWA: Multidimensional FWA

\section{Prediction models and statistical analysis}

PCV prediction models were built using a combination of stepwise and sparse logistic regression. See Supplemental Materials for a detailed description of the parameter selection procedure. Prediction performance was measured as the area under the receiver operating characteristic (ROC) curve (AUC). Complexity parameters were first individually scored in terms of predictive performance. Then, parameters were divided into 4 groups, based on their computational domain (time or frequency) and the number of leads involved in the computation (one lead or multiple leads). Best predicting parameter models were compared to the prediction performance obtained by using conventional clinical and echocardiographic predictors. Model prediction performance was cross-validated using 5 -fold data partitioning with repeated (20 times) random subsampling. Differences in model performance were 
assessed with a Student's t-test with a significance threshold of $p=0.05$. The association between ECG complexity parameters and clinical parameters, and the risk of progression to persistent AF was investigated using sparse Cox proportional hazards models. ${ }^{8}$ Differences in hazard model fit quality were assessed using a likelihood ratio test with a significance threshold of $p=0.05$.

All computations were performed in MATLAB (MATLAB and Statistics Toolbox Release 2014a, The MathWorks, Inc., Natick, Massachusetts, United States), using custom made software and the Glmnet for MATLAB toolbox for elastic net regression.

\section{Results}

\section{Prediction using one parameter derived from one lead}

Single lead parameter results are listed in Supplemental Table 1.

The best predictor for successful PCV was lower dominant frequency (DF), with maximum single lead AUC $(0.66,95 \%$ confidence interval [0.64-0.67], sensitivity 78[76-79]\%, specificity 45[43-48]\%) at lead II. All other significant predictors in the frequency domain obtained lower performance with an AUC below 0.60. Best predictor in the time-domain was sample entropy (SAE) at lead II with comparable, if not slightly lower performance (AUC 0.63 [0.62$0.65]$, sensitivity $86[85-87] \%$, specificity $33[31-35] \%))$.

\section{Prediction using one parameter derived from multiple leads}

Multidimensional parameter results are listed in Supplemental Table 2.

This analysis focused on the parameters that were computed using information derived from multiple leads, but expressed the complexity of those multiple leads as a single parameter value. Maximum predictive power was observed using multidimensional DF (MDF) (AUC 0.64 [0.62-0.66], derived from leads $V_{(1,2,4,5,6)}$, sensitivity $84[82-86] \%$, specificity $\left.37[35-39] \%\right)$ and spectral variability (SV) (AUC 0.64 [0.62-0.66], all leads, sensitivity 77[76-79]\%, specificity $48[45-50] \%)$. In the time domain, none of the significant predictors reached an AUC above 0.60 .

\section{Prediction using a combination of ECG parameters}

Results for prediction models consisting of a combination of ECG parameters are listed in Table 2. 
Chapter 4

\begin{tabular}{|c|c|c|c|}
\hline Group & Parameters & Leads or signal & $\begin{array}{c}\text { AUC } \\
{[95 \% \mathrm{Cl}]}\end{array}$ \\
\hline Single lead & $\mathrm{DF}$ & II & $0.72[0.70-0.73]$ \\
\hline \multirow[t]{2}{*}{ Frequency domain } & Ol & III & \\
\hline & SE & I & \\
\hline Single lead & SAE & II & $0.72[0.71-0.74]$ \\
\hline \multirow[t]{2}{*}{ Time domain } & FWA & $\mathrm{aVF}, \mathrm{V}_{1}$ & \\
\hline & FWP MAW & $\mathrm{V}_{2}$ & \\
\hline Multiple leads & MDF & $V_{(1,2,4,5)}, V_{(1,2,4,5,6)}$ & $0.71[0.69-0.72]$ \\
\hline \multirow[t]{2}{*}{ Frequency domain } & $\mathrm{MOI}$ & $\mathrm{V}_{(3,4)}, \mathrm{V}_{(3,5)}, \mathrm{V}_{(2,4,5,6)}$ & \\
\hline & SV & All leads & \\
\hline Multiple leads & MFWA & AA & $0.61[0.60-0.63]$ \\
\hline Time domain & $\mathrm{CV}$ & AA & \\
\hline Combined & \multicolumn{2}{|c|}{$\begin{array}{l}\mathrm{DF}(\mathrm{II}), \mathrm{SE}(\mathrm{I}), \mathrm{FWA}\left(\mathrm{aVF}, \mathrm{V}_{1}\right), \mathrm{MOI} \\
\left(\mathrm{V}_{(3,4)}, \mathrm{V}_{(3,5)}\right), \mathrm{SV}, \mathrm{MFWA}\end{array}$} & $0.78[0.76-0.79]$ \\
\hline
\end{tabular}

Table 1: Best performing parameter models for single and multidimensional ECG parameters in the frequency- and time-domain. DF: Dominant Frequency, OI: Organization Index, SE: Spectral Entropy, MDF/MOI: Multidimensional DF/OI, SV: Spectral Variability, SAE: Sample Entropy, FWA: Fibrillation Wave Amplitude, FWP MAW: Fibrillation Wave Power of the Main Atrial Wave, MFWA: Multidimensional FWA 
The best model containing a combination of frequency-domain parameters computed on a single lead improved performance from an AUC of 0.66 [0.640.67 ] to $0.72[0.70-0.73]$, sensitivity $75[73-77] \%$, specificity $54[52-57] \%$ $(p<0.001)$, by adding OI (lead III) and SE (lead I) to the best single lead parameter DF (lead II). In the time-domain prediction improved by extending the best performing single lead parameter SAE (lead II) with FWA (lead aVF and $V_{1}$ ) and FWP (lead $V_{2}$ ), from an AUC of 0.63 [0.62-0.65] to 0.72 [71-74], sensitivity $83[81-84] \%$, specificity $48[46-51] \% \quad(p<0.001)$. Combining multidimensional parameters produced similar results in the frequencydomain, with a 6-parameter model increasing the AUC from 0.64 [0.62-0.66] to 0.71 [0.69-0.72], sensitivity 95[94-96]\%, specificity 29[27-31]\% $(p<0.001)$. Combining multidimensional time-domain parameter did not improve predictive. Combining the best predicting parameters of each group into a single model further improved prediction performance (AUC 0.78 [0.76-0.79], sensitivity $80[79-82] \%$, specificity $60[57-62] \%)$. A selection of cross-validated ROC curves is depicted in Figure 2. Supplemental Figure 1 shows the effect of combining parameters within each parameter group on prediction performance.

\section{Prediction using clinical parameters and ECG parameters}

An overview of the predictive performance obtained when combining clinical patient characteristics and ECG parameters can be found in Table 3 and Figure 3.

The predictive capability of clinical parameters alone was limited, using weight and right atrial volume (RAV) (0.68 [0.66-0.70], sensitivity 87[86-89]\%, specificity 35[32-37]\%). Predictive performance of the models based on the 4 ECG parameter groups was superior, except for the multidimensional timedomain parameter model. Adding the ECG parameters from the best performing group models to the clinical parameters significantly enhanced predictive performance in all cases, again except for the multidimensional time-domain parameter model. Maximum AUC was reached by combining clinical and single lead frequency-domain parameters (AUC 0.81 [0.79-0.82], sensitivity $83[81-85] \%$, specificity $64[61-67] \%)$. 


\section{Chapter 4}

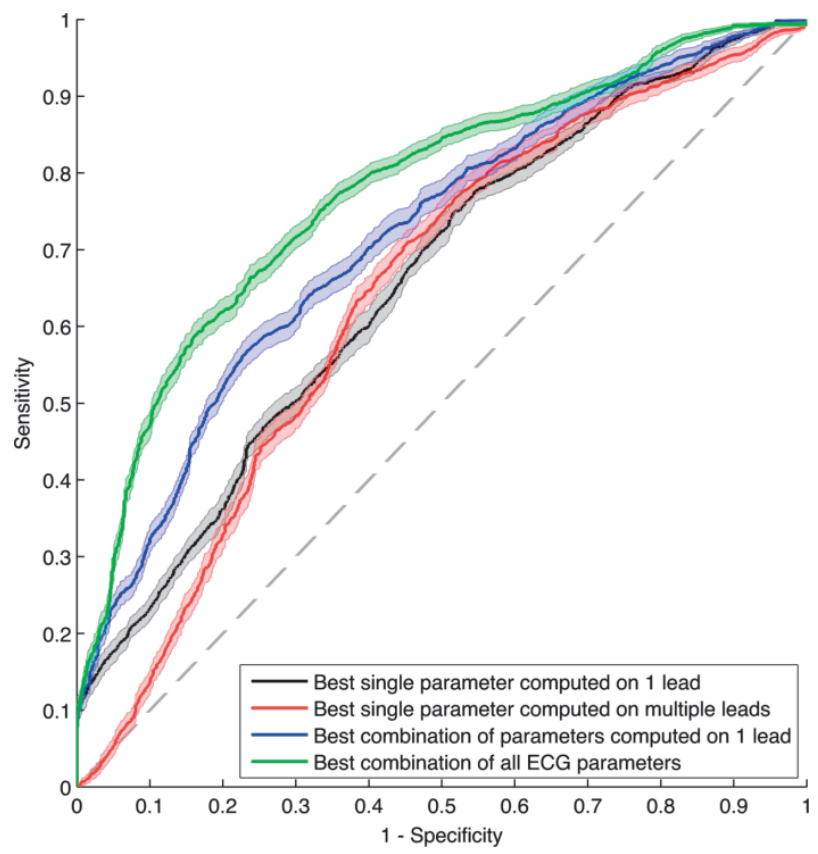

Figure 2: Cross-validated ROC curves of various ECG parameter models. The band around each curve indicates the $95 \%$ confidence interval of the sensitivity for a given specificity.

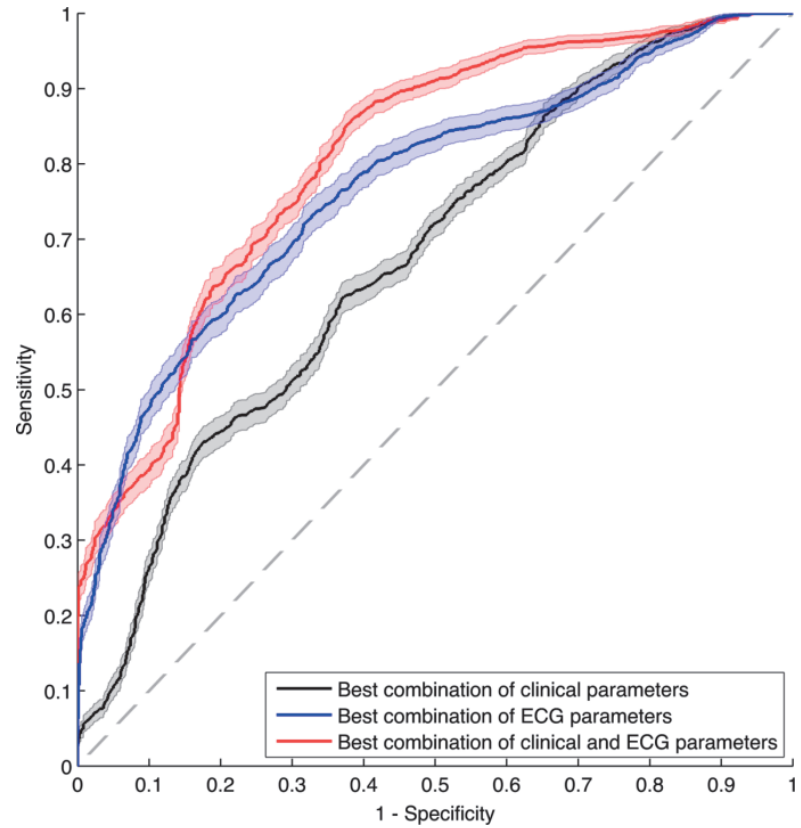

Figure 3: Cross-validated ROC curves showing the prediction performance of the best model consisting only of clinical parameters, only of parameters computed on the ECG, and a combination clinical and ECG parameters. The bands around the curves indicate the $95 \%$ confidence interval of the sensitivity for a given specificity. 


\begin{tabular}{|c|c|c|c|}
\hline Parameter model & $\begin{array}{l}\text { AUC on subset } \\
(n=139)\end{array}$ & $\begin{array}{l}\text { AUC Clinical \& } \\
\text { ECG Parameters }\end{array}$ & P-value \\
\hline $\begin{array}{l}\text { Clinical parameters } \\
\text { (Weight, RAV) }\end{array}$ & $0.68[0.66-0.70]$ & $\mathrm{N} / \mathrm{A}$ & N/A \\
\hline Single lead & $0.75[0.73-0.77]$ & $0.81[0.79-0.82]$ & $<0.001$ \\
\hline Frequency domain & & & $(<0.001)$ \\
\hline Single lead & $0.73[0.71-0.74]$ & $0.77[0.75-0.78]$ & $<0.001$ \\
\hline Time domain & & & $(<0.001)$ \\
\hline Multiple leads & $0.73[0.71-0.74]$ & $0.77[0.75-0.78]$ & $<0.001$ \\
\hline Frequency domain & & & $(<0.001)$ \\
\hline Multiple leads & $0.59[0.57-0.61]$ & $0.67[0.65-0.68]$ & 0.289 \\
\hline Time domain & & & $(<0.001)$ \\
\hline Best ECG model & $0.78[0.76-0.79]$ & $0.78[0.76-0.80]$ & $\begin{array}{l}<0.001 \\
(0.473)\end{array}$ \\
\hline
\end{tabular}

Table 2: Predictive performance of clinical parameters and the added value of the best performing single lead and multidimensional ECG parameters models, as determined on the full data set (see Table 2). P-values denote the comparison between the model consisting of only clinical parameters and the specific combination. P-values between brackets signify the difference between the model consisting of ECG parameters and the combined model of clinical (weight and RAV) and ECG parameters.

\section{Risk of progression to persistent AF}

Out of the 201 patients for whom follow-up was available, 38 (19\%) developed persistent AF between the date of PCV and March 2015 (median time to persistent AF: 408 days, interquartile range (IQR): 171-822 days). Table 4 contains the significant hazard ratios (HR) for individual clinical and ECG complexity parameters. Age, BMI, left atrial diameter (LAD), RAV, left ventricular end systolic diameter (LVESD) and ejection fraction (LVEF) showed small, but significant hazard ratios. As expected from previous studies, an elevated HATCH score indicated a significantly higher risk for progression to persistent AF.[13] Unsuccessful PCV was not a significant hazard (HR 1.58, 95\% confidence interval $(\mathrm{Cl}) 0.82-3.06, p=0.17)$. ECG complexity parameters were only significant for DF and FWA. Both a higher DF and - surprisingly - a higher FWA were associated with a larger risk of developing persistent AF. Figure 4 depicts Kaplan-Meier curves for four dichotomized parameters, showing that obesity $\left(B M I>30 \mathrm{~kg} / \mathrm{m}^{2}\right)$, an enlarged left atrium (LAD>41 mm), faster atrial rate $(D F>5.7 \mathrm{~Hz}$ ), and higher $\mathrm{f}$-wave amplitude (FWA $>0.06 \mathrm{mV}$ ) were linked to 
Chapter 4

an increased risk of AF progression. Progression was significantly faster for patients with FWA $>0.06 \mathrm{mV}$ (median time to persistent AF 296 days vs. 796 days, $\mathrm{p}=0.03$ ).

Combing parameters showed that the risk of progression to persistent AF was best explained by LAD alone, when considering only clinical parameters. ECG complexity parameters modelled progression best using a combination of DF (lead $\mathrm{aVL}$ ) and FWA (lead $\left.\mathrm{V}_{1}\right)(\mathrm{n}=201, \mathrm{DF}(\mathrm{aVL})$ : HR 1.45, Cl 1.08-1.94, $\mathrm{p}=0.01$; $\mathrm{FWA}\left(\mathrm{V}_{1}\right)$ : HR 1.16, $\left.\mathrm{Cl} 1.05-1.27, \mathrm{p}<0.01\right)$. Adding $\mathrm{DF}(\mathrm{avL})$ or FWA $\left(\mathrm{V}_{1}\right)$ to the best model containing only clinical parameters both improved model quality ( $p=0.05$ or 0.02 respectively).

\begin{tabular}{|c|c|c|c|}
\hline \multirow[b]{2}{*}{ Parameter } & \multirow[b]{2}{*}{ Increment } & \multicolumn{2}{|c|}{ Hazard ratios $(95 \% \mathrm{CI})$} \\
\hline & & Unadjusted & Adjusted for Sex and \\
\hline & & & Age \\
\hline Age & 1 year & $1.03(1.01-1.06)+$ & $\mathrm{N} / \mathrm{A}$ \\
\hline BMI $(n=159)$ & $1 \mathrm{~kg} / \mathrm{m}^{2}$ & $1.09(1.03-1.17) \ddagger$ & $1.10(1.03-1.18) \ddagger$ \\
\hline LAD (n=155) & $1 \mathrm{~mm}$ & $1.12(1.05-1.19) \ddagger$ & $1.11(1.04-1.18) \ddagger$ \\
\hline $\operatorname{RAV}(n=141)$ & $5 \mathrm{ml}$ & $1.11(1.03-1.21) \dagger$ & $1.12(1.02-1.22) \dagger$ \\
\hline LVESD $(n=158)$ & $1 \mathrm{~mm}$ & $1.06(1.00-1.11) \dagger$ & $1.08(1.02-1.14) \ddagger$ \\
\hline $\operatorname{LVEF}(n=160)$ & $-1 \%$ & $1.06(1.02-1.11) \ddagger$ & $1.06(1.02-1.10) \ddagger$ \\
\hline HATCH $(n=184)$ & 1 point & $1.43(1.10-1.86) \ddagger$ & $1.27(0.92-1.76)$ \\
\hline DF (III) & $1 \mathrm{~Hz}$ & $1.57(1.17-2.10) \dagger$ & $1.65(1.24-2.19) \ddagger$ \\
\hline $\mathrm{DF}(\mathrm{aVL})$ & $1 \mathrm{~Hz}$ & $1.50(1.14-1.99) \ddagger$ & $1.64(1.24-2.16) \ddagger$ \\
\hline DF (aVF) & $1 \mathrm{~Hz}$ & $1.46(1.11-1.92) \ddagger$ & $1.53(1.17-2.00)+$ \\
\hline $\mathrm{DF}\left(\mathrm{V}_{4}\right)$ & $1 \mathrm{~Hz}$ & $1.37(1.03-1.87)+$ & $1.34(0.99-1.83)$ \\
\hline $\operatorname{FWA}\left(V_{1}\right)$ & $0.01 \mathrm{mV}$ & $1.17(1.07-1.29) \ddagger$ & $1.16(1.06-1.27) \ddagger$ \\
\hline
\end{tabular}

Table 3: Significant hazard ratios for risk of progression to persistent AF. $+p<0.05, \neq p<0.01$. BMI: body mass index, $\mathrm{Cl}$ : Confidence interval, DF: dominant frequency, FWA: F-wave amplitude, LAD: left atrial diameter, LVEF: left ventricular ejection fraction, LVESD: left ventricular end systolic diameter, RAV: right atrial volume. 

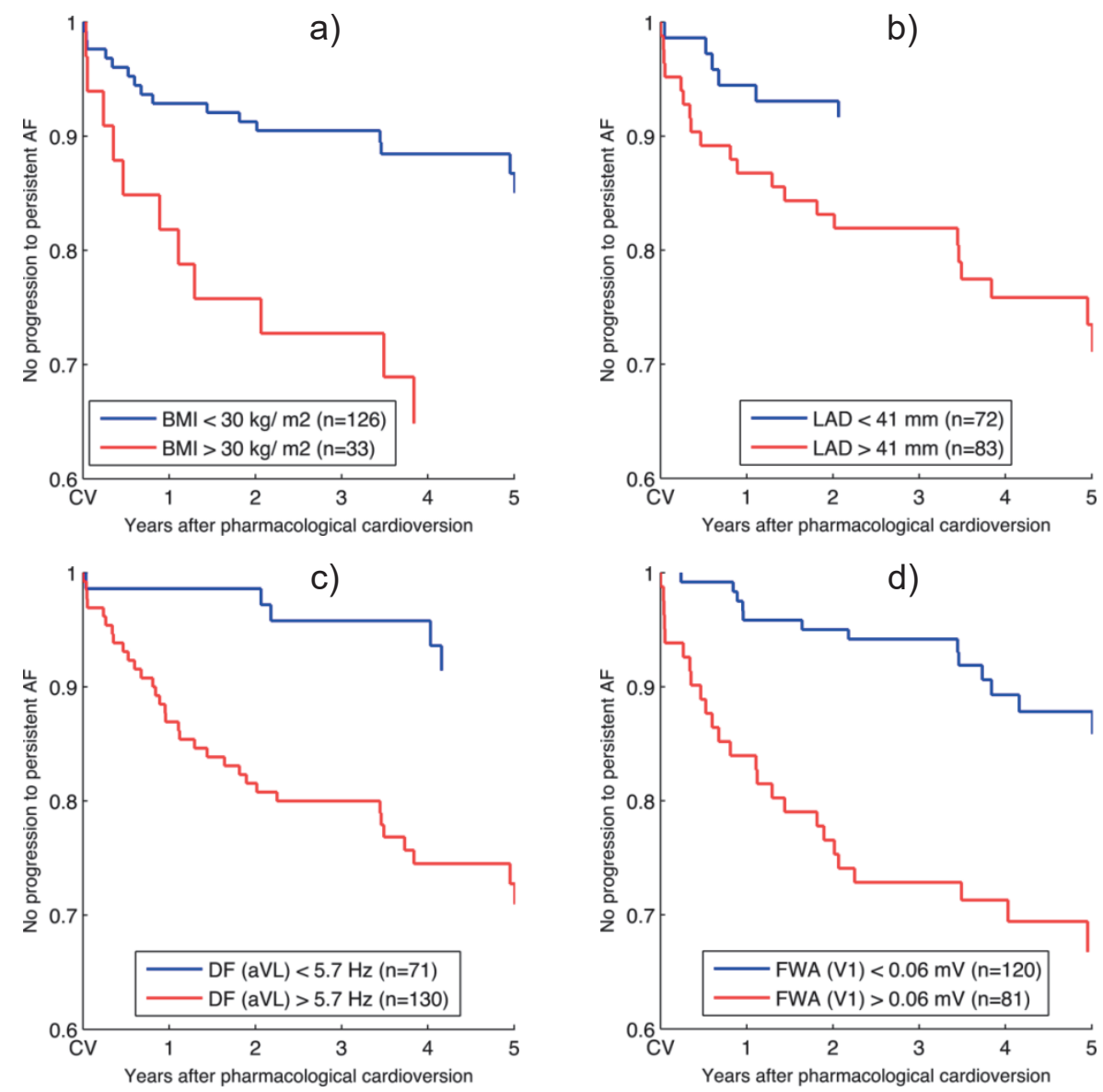

Figure 4: Kaplan-Meier curves for the risk of progression to persistent atrial fibrillation after the pharmacological cardioversion attempt for patients with a) body mass index (BMI) $>30 \mathrm{~kg} / \mathrm{m}^{2}$ (HR 2.97), b) left atrial diameter (LAD) $>41 \mathrm{~mm}$ (HR 2.65), c) dominant frequency (DF) $>5.7 \mathrm{~Hz}$ on lead aVL (HR 4.16), and d) F-wave amplitude (FWA) $>0.06 \mathrm{mV}$ (HR 3.25). All HRs $p<0.01$.

\section{Discussion}

\section{EGC parameters as predictors of pharmacological cardioversion outcome}

The results of the single and multidimensional ECG lead analysis show that the 12-lead ECG contains valuable information related to successful PCV of recent onset AF. Patients with a lower DF were more likely to respond to treatment, which corroborates the findings of Choudhary et al. ${ }^{9}$ who showed that recent onset AF patients with a lower DF were more likely to spontaneously cardiovert. Also in patients with persistent AF a lower DF was found to predict successful electrical CV outcome. ${ }^{10}$ Moreover, single lead measures of 
organization of atrial rate (OI and $\mathrm{SE}$ ) indicate that a higher degree of organization favours successful PCV. Overall, differences between successful and unsuccessful PCV were small, but plausible given the interpretation of frequency-domain parameters. In the time-domain, a lower SAE, a measure of temporal irregularity of the atrial activity, was associated with higher chance of PCV success, which is consistent with the results of Alcaraz et al. in a study on prediction of spontaneous cardioversion. ${ }^{11}$

Multidimensional parameters that compute one complexity indicator from multiple leads are a logical extension of single lead analysis. Incorporating spatial differences among leads and capturing inter-lead variability as an additional measure of complexity, could lead to a more robust estimate of AF complexity. The results from the multidimensional parameter analysis partially confirm this. While DF computed on one of the precordial leads only gave a significant result on lead $\mathrm{V}_{1}$, the multidimensional extension MDF performed better, with significant results for many combinations of precordial leads. Maximum performance of MDF was however still lower than the performance of single lead DF on limb lead II (AUC 0.64 vs. 0.66). We did not notice an important role of left atrial content in this patient population as indicated by Uldry et al. in their study on discriminating persistent and long-standing persistent AF. ${ }^{12}$ The most significant multidimensional parameter differences were observed in a mix of right- and left-oriented precordial leads. Overall however, predictive performance of a single parameter was moderate. This is likely due to subtle differences in AF complexity between patients with recent onset AF. Furthermore, in this early stage of AF development the process of electrical remodelling is still on-going and may have an important effect on the success of PCV. The degree of electrical remodelling increases with AF duration. Although precise AF duration is difficult to assess in the majority of recent onset AF patients, DF may serve as a surrogate parameter for AF duration in these patients.

Combining several complexity parameters in a prediction model significantly improved prediction, regardless of whether these different parameter values were calculated from single lead or multiple leads. Worthwhile noting is that the best combination of frequency-domain parameters computed on a single lead was composed completely of limb leads I, II and III, with a strong role of DF at lead II, again suggesting the need to include leads that contain both right and left atrial activity.

Added predictive value of ECG parameters compared to clinical information 
The ability of clinical parameters, including echocardiographic parameters, to predict successful outcome of PCV was limited. Combinations of ECG parameters performed better on the subset of patients with complete clinical and echocardiographic data records. Combining ECG and clinical parameters further improved prediction. This implies that features extracted from the ECG contain complementary information to the available clinical characteristics in this patient population. In particular, single lead frequency-domain parameters improved prediction.

\section{Non-invasive complexity and risk of progression to persistent AF}

Interestingly, both clinical as well as ECG complexity parameters were associated with risk of progression to persistent AF. Clinical parameters like age, BMI and HATCH score, and echocardiographic parameters like LAD, RAV and LVEF were indicators for an increased risk of progression to persistent AF, which is in line with previous findings. $[13,18]$ From the set of ECG parameters only parameters computed on a single lead showed significant hazard ratios, namely DF and FWA. The threshold of $5.7 \mathrm{~Hz}$ computed for DF to produce the survival curve in Figure $4 \mathrm{c}$ is very comparable to the AFR threshold of $<350$ fibrillations per minute $(5.8 \mathrm{~Hz})$ found by Choudhary et al. ${ }^{9}$ associated with a significant increase in the likelihood of spontaneous cardioversion of recent onset AF within 18 hours. One could argue that patients that are not likely to spontaneously cardiovert have a higher risk to develop persistent AF, due to more electrical and, eventually, structural remodelling caused by prolonged episodes of AF. On the other hand, Mochalina et al. ${ }^{13}$ reported no predictive value of DF in a comparable cohort of patients undergoing PCV with vernakalant. A possible explanation for this discrepancy is that in their study only lead $V_{1}$ was analyzed, as opposed to all 12 ECG leads in the present study. Alternatively, differences in the anti-arrhythmic mechanism of flecainide and vernakalant may affect the predictive performance of complexity parameters. The role of FWA on $V_{1}$ in the development of persistent AF in this patient cohort is more challenging to interpret: a higher FWA was associated with a higher risk for persistent AF, while the inverse relation was found for FWA in the prediction of successful PCV. The amplitude of fibrillation waves that are visible on an ECG is influenced by both the atrial mass and the degree of complexity of AF. On the one hand, patients with an organised AF pattern have a larger simultaneously activated atrial mass and therefore larger vectors in a certain direction. Larger electrical vectors are expected to produce larger $\mathrm{f}$ waves, potentially explaining higher success rates of PCV. On the other hand, patients with atrial dilatation have more atrial mass and therefore could produce larger $\mathrm{f}$-waves. Patients with atrial dilatation are known to progress 
into persistent AF, also indicated by our results.[13] Certainly, the implications of FWA for long-term rhythm outcome warrant further investigation.

\section{ECG parameters in clinical decision-making}

We showed that non-invasive atrial complexity parameters derived from a standard 12-lead ECG improve prediction of successful PCV in patients with recent onset AF. These parameters can be computed automatically while recording an ECG. Correctly predicting patients in whom AF is likely to terminate using drugs is of relevance in the emergency department. In patients with low complexity, one could wait for spontaneous CV or restoration of SR (for example using intravenously administered AADs) without continuous antiarrhythmic drug treatment might be sufficient, avoiding adverse effects of chronic AAD therapy. ${ }^{4}$ For patients reporting at the emergency department with a complex AF pattern, a more aggressive rhythm control strategy may be necessary to prevent recurrences and reduce the risk of progression to persistent AF, by either continuous AAD treatment or early catheter ablation. Early recognition of these patients with early treatment might result in improved success percentages and prevent future cardiovascular complications. ${ }^{14}$

\section{Limitations}

The retrospective nature of this study had implications for the availability and quality of clinical information and ECG signals. Echocardiography was not recorded at the same time as the ECG, but selecting an available echocardiography within a year produced similar results compared to a narrower timeframe (see Supplemental Materials). ECG signals were not recorded with the intention to analyse AF complexity but rather to diagnose the arrhythmia, meaning that quality was varying and recording duration was limited to 10 seconds. This does however reflect everyday clinical practice.

\section{Conclusions}

AF complexity parameters determined from 12-lead ECGs are superior to common clinical predictors in predicting successful PCV in patients with recent onset AF. Combining ECG and clinical parameters generally improved prediction, especially by including single lead frequency-domain parameters. Notably, both clinical characteristics as well as ECG complexity parameters can predict progression to persistent $A F$, which may guide individualized rhythm control strategies in the future. 


\section{References}

1. Allessie MA, de Groot NM, Houben RP, Schotten U, Boersma E, Smeets JL, Crijns HJ. Electropathological substrate of long-standing persistent atrial fibrillation in patients with structural heart disease: longitudinal dissociation. Circulation Arrhythmia and Electrophysiology 2010;3:606-615.

2. de Groot NM, Houben RP, Smeets JL, Boersma E, Schotten U, Schalij MJ, Crijns $\mathrm{H}$, Allessie MA. Electropathological substrate of longstanding persistent atrial fibrillation in patients with structural heart disease: epicardial breakthrough. Circulation 2010;122:1674-1682.

3. Cuculich PS, Wang Y, Lindsay BD, Faddis MN, Schuessler RB, Damiano RJ, Jr., Li L, Rudy Y. Noninvasive characterization of epicardial activation in humans with diverse atrial fibrillation patterns. Circulation 2010;122:1364-1372.

4. Camm AJ, Kirchhof P, Lip GY, et al. Guidelines for the management of atrial fibrillation: the Task Force for the Management of Atrial Fibrillation of the European Society of Cardiology (ESC). European Heart Journal 2010;31:23692429.

5. Lankveld TA, Zeemering S, Crijns HJ, Schotten U. The ECG as a tool to determine atrial fibrillation complexity. Heart 2014;100:1077-1084.

6. Schotten $U$, Maesen B, Zeemering S. The need for standardization of timeand frequency-domain analysis of body surface electrocardiograms for assessment of the atrial fibrillation substrate. Europace 2012;14:1072-1075.

7. Bonizzi P, Zeemering S, Karel JM, Di Marco LY, Uldry L, Van Zaen J, Vesin JM, Schotten U. Systematic comparison of non-invasive measures for the assessment of atrial fibrillation complexity: a step forward towards standardization of atrial fibrillation electrogram analysis. Europace 2015;17:318-325.

8. Simon N, Friedman J, Hastie T, Tibshirani R. Regularization Paths for Cox's Proportional Hazards Model via Coordinate Descent. Journal of Statistical Software 2011;39:1-13.

9. Choudhary MB, Holmqvist F, Carlson J, Nilsson HJ, Roijer A, Platonov PG. Low atrial fibrillatory rate is associated with spontaneous conversion of recentonset atrial fibrillation. Europace 2013;15:1445-1452.

10. Lankveld T, de Vos CB, Limantoro I, Zeemering S, Dudink E, Crijns HJ, Schotten U. Systematic Analysis of ECG Predictors for Sinus Rhythm Maintenance Following Electrical Cardioversion for Persistent Atrial Fibrillation. Heart Rhythm2016.

11. Alcaraz R, Rieta JJ. Sample entropy of the main atrial wave predicts spontaneous termination of paroxysmal atrial fibrillation. Medical Engineering \& Physics 2009;31:917-922.

12. Uldry L, Van Zaen J, Prudat Y, Kappenberger L, Vesin JM. Measures of spatiotemporal organization differentiate persistent from long-standing atrial fibrillation. Europace 2012;14:1125-1131. 


\section{Chapter 4}

13. Mochalina N, Juhlin T, Ohlin B, Carlson J, Holmqvist F, Platonov PG. Predictors of successful cardioversion with vernakalant in patients with recent-onset atrial fibrillation. Annals of Noninvasive Electrocardiology 2015;20:140-147.

14. Nattel S, Guasch E, Savelieva I, et al. Early management of atrial fibrillation to prevent cardiovascular complications. European Heart Journal 2014;35:14481456. 


\section{Supplements}

\section{Methods}

\section{Non-invasive AF Complexity parameters}

\section{Spectral parameters}

The frequency content of each lead was determined by computing the spectrum using 1) the (fast) Fourier transform of the extracted atrial signal, 2) Welch's power spectral density estimate (3 segments, 1024 points, 50\% overlap), and 3) the compressed spectrum (CS) ${ }^{1}$ using the original ECG signal.

The dominant atrial frequency (DF) was defined as the frequency with the largest power within the $3-12 \mathrm{~Hz}$ band. The organization index (OI) of the spectrum was defined as the relative contribution of the 2 largest peaks to the total spectral power. A low value of OI indicates high complexity. Spectral entropy (SE) is the application of Shannon's entropy to the frequency distribution and can be interpreted as a measure of uniformity. A high value of SE indicates high complexity.

Single lead spectral analysis can be extended to a multidimensional analysis that incorporates spectral information from multiple leads using the so-called spectral envelope. The spectral envelope describes the shared spectral characteristics of a multidimensional signal. ${ }^{2}$ This means that the spectral information from multiple leads is represented in a single spectrum. From the spectral envelope, the same three spectral parameters were derived: multidimensional dominant frequency (MDF), multidimensional spectral organization index (MOI) and multidimensional spectral entropy (MSE). ${ }^{3}$ MDF, $\mathrm{MOI}$ and MSE were computed on the spectral envelope of all possible combinations of 2 or more precordial leads, as opposed to only pairs of leads in Uldry et al. ${ }^{3}$

\section{Fibrillation wave amplitude}

The amplitude of fibrillation waves was determined in two ways: automatic annotation of $f$-waves in a single lead by peak detection, followed by amplitude computation (FWA), comparable to the manual annotation method used by Nault et al. ${ }^{4}$, and - analogous to the computation of spectral complexity - a signal envelope approach that computes a multidimensional $\mathrm{f}$ wave amplitude on multiple leads (MFWA). ${ }^{5}$ MFWA was computed on both the AA signal and TQ segments. A low value of FWA or MFWA indicates high complexity. 
Chapter 4

\section{Sample entropy}

Sample entropy (SAE) is a time-domain parameter that quantifies the irregularity of a signal by searching for similar segments of a certain length. As proposed by Alcaraz et al. ${ }^{6}$ SAE was computed on the main atrial wave (MAW) of each lead. The MAW is the signal resulting from filtering the atrial signal centred around the dominant frequency with a $3 \mathrm{~Hz}$ bandwidth. A high value of SAE indicates high complexity. Additional parameters related to the MAW are the $f$-wave power of the MAW (FWP MAW), with similar interpretation as FWA, and the relative sub-band energy $(\mathrm{RHE})^{6}$, computed as the relative energy present in the first and second harmonics of the MAW. A low value of RHE indicates high complexity.

\section{Principal component analysis}

Another multidimensional approach to AF complexity quantification is principal component analysis (PCA), which expresses the information from all 12 leads in a number of linearly uncorrelated components that essentially describe the amount of variance between the leads. Complexity measures based on PCA included were spatial complexity $k_{0.95}$, the number of components required to describe $95 \%$ of the variance in all 12 leads, and spatio-temporal stationarity (NMSE), the degree in which the three major signal components vary over time. ${ }^{7}$ A high value of $k_{0.95}$ and NMSE indicates high complexity. Additional measures of spatial complexity $\mathrm{C}$ and variability of spatial complexity CV were also included. C defines spatial complexity as the relative signal variance, excluding the three major components. ${ }^{8}$ Frequency domain parameters derived from PCA were spectral concentration SC and spectral variability $\mathrm{SV}^{8}$, where SC quantifies the concentration of the spectral power around the dominant frequency and SV the temporal variation of the SC. A low value of SC or a high value of SV indicates high complexity. PCA parameters were computed on both the AA signal and the TQ segments. 


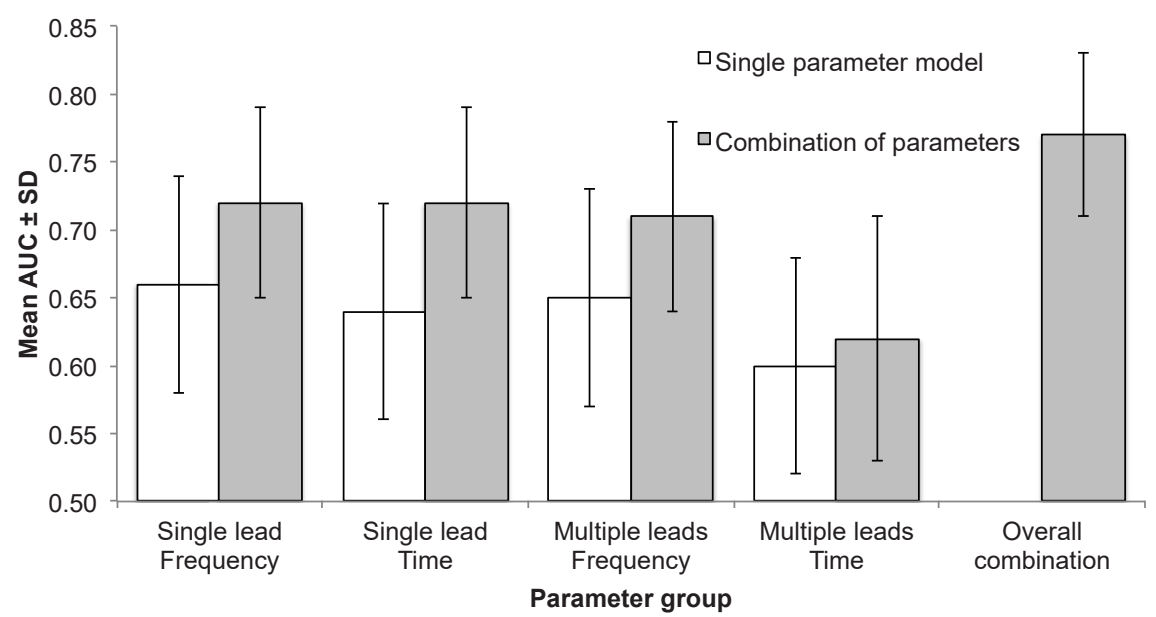

Figure 1: Prediction performance of different types of complexity parameters computed on one lead and multiple leads, in the frequency-domain and the time-domain. Performance is expressed as the mean AUC $\pm S D$ for each of the 4 groups (parameter computed in the frequency/time-domain and on one/multiple leads). For every group the best performing single parameter model AUC is given, as well as AUC of the best combination of parameters belonging to the same group.

\section{Parameter selection via elastic net logistic regression}

In several cases, the number of candidate parameters in the logistic regression model makes it infeasible to iterate over all possible parameter combinations to select the overall best performing model. Parameter selection using stepwise logistic regression has the disadvantage that it is dependent on the order in which parameters are added or removed from the prediction model. Stepwise parameter selection is also affected by parameter correlation. To select dominant parameters from a large set of candidate parameters, and to overcome the limitations of stepwise methods we applied an approach that combines information from classical stepwise logistic regression and elastic net logistic regression. Elastic net regression is based on mixed $\ell_{1} / \ell_{2}$-norm regularization of the parameter coefficients in the criterion function of the regression model at hand. This regularization aims to minimize the number of non-zero parameter coefficients in the estimated model. Given a certain output data $y$ of length $N$, in the case of logistic regression the objective is to minimize the model deviance $D(y, \theta)=-2\left(\log (p(y \mid \theta))-\log \left(p\left(y \mid \theta_{s}\right)\right)\right)$, where the vector $\vartheta$ contains the parameter coefficients and $\vartheta_{s}$ denotes the parameter vector of the saturated model. The formulation for the elastic net logistic regression problem is 
$\min _{\theta}\left(\frac{1}{N} D(y, \theta)+\lambda P_{\alpha}(\theta)\right)$, with

$P_{\alpha}(\theta)=\frac{(1-\alpha)}{2}\|\theta\|_{2}^{2}+\alpha\|\theta\|_{1}$.

The two regression tuning parameters are lambda $(\lambda)$ and alpha $(\alpha)$. Lambda determines the strength of the regularization of the parameter coefficients, while alpha (a value between 0 and 1 ) controls the balance between penalizing either the $l_{2}$ - and/or the $\ell_{1}$-norm of the coefficient vector ${ }^{9}$.

Several steps of the parameter selection procedure are outlined in Figure 2. In the analysis shown there the set of parameters under investigation was the group of parameters computed on 1 lead in the frequency domain (DF, OI, SE and $\mathrm{RHE}$ ). Figure 2a) and b) show the elastic net estimation result for a fixed value of alpha (alpha $=0.5$ ). The choice of lambda influences the estimated parameter coefficients and the deviance of the estimated model. A commonly accepted choice for lambda is the value that corresponds to a model deviation that lies within 1 standard deviation of the cross-validated minimum deviation. These lambda values are indicated with a green (minimum deviation) and a blue line (minimum deviation +1 standard deviation). The choice of alpha also determines the number of parameters that are selected. For alpha $=1$ the algorithm corresponds the Lasso algorithm, which tends to select one parameter from a group of correlated parameters, but for alpha values between 0 and 1 , the elastic net algorithm will include more correlated parameters. Therefore a range of alpha (between 0.1 and 1) was investigated and for each value of alpha the non-zero parameter coefficients were stored (see Figure 2c)). Parameters that appeared in any of the models computed with this range of alpha were considered potential candidates for the final logistic regression model. In this case the parameters DF(on leads II, aVR and $\mathrm{V}_{4}$ ), OI (leads I and III) and RHE (lead I) were selected. As an additional step, parameters were also selected through forward stepwise logistic regression ( $P$ $<0.05$ for significant deviance improvement by adding a parameter). In this case selected parameters were DF (lead II), OI (III) and SE (I). The union of the parameters selected by the two regression methods was then taken to iterate over all possible combinations of parameters to find the model with the best prediction performance. Figure $2 \mathrm{~d}$ ) shows the result of this last step. The model performance increased by adding more parameters, but reached a maximum at a model containing 3 parameters (DF (II), OI (III) and SE(I)). 

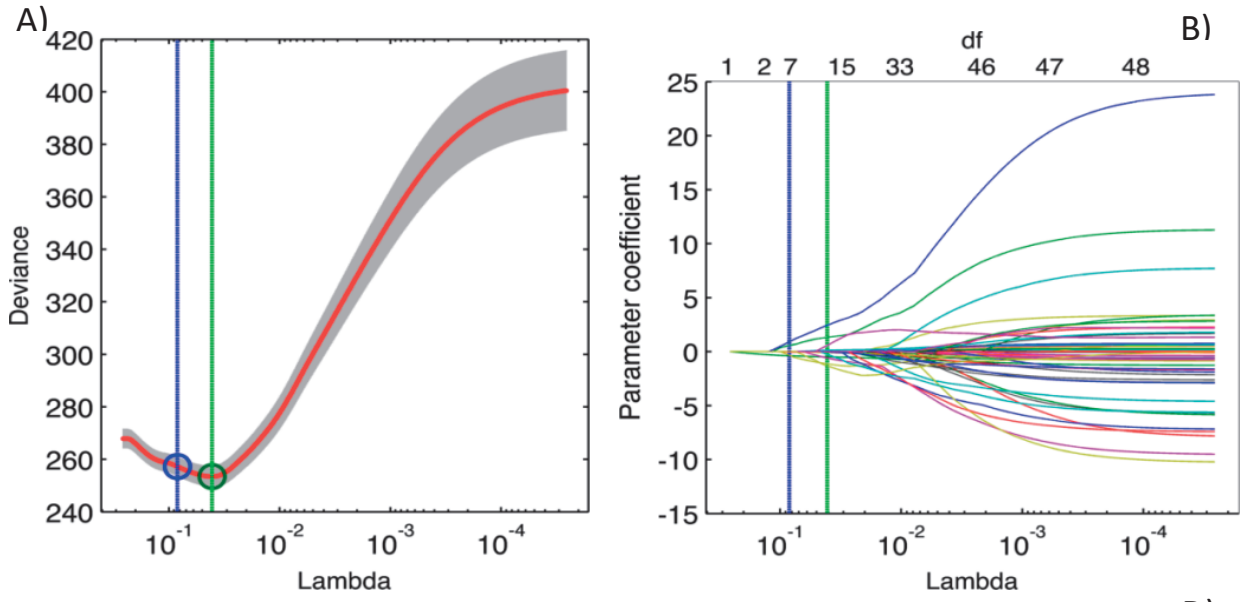

C)
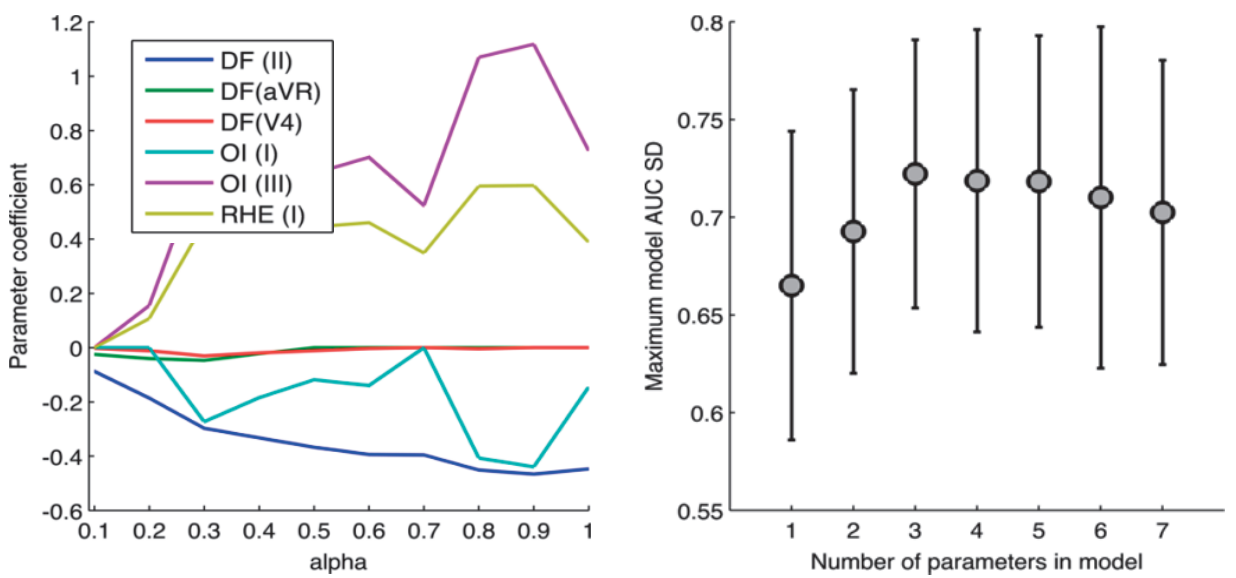

Figure 2: Parameter selection via elastic net logistic regression (parameters computed on a single lead, frequency-domain). The upper plots show the result of the analysis for alpha=0.5, with in a) the cross-validated deviance as a function of lambda and in b) the parameters coefficients ( $\mathrm{df}$ indicates the number of non-zero parameter coefficients). The green line/circle marks the choice of lambda that minimizes the deviance, the blue line/circle marks the solution that is within 1 standard deviation. Panel c) shows the non-zero parameters coefficients selected by the elastic net regression as a function of alpha. Panel d) contains the result for the crossvalidated maximum AUC for models composed of a specific number of candidate parameters, defined by union of the stepwise regression and elastic net parameter selection. 


\section{Chapter 4}
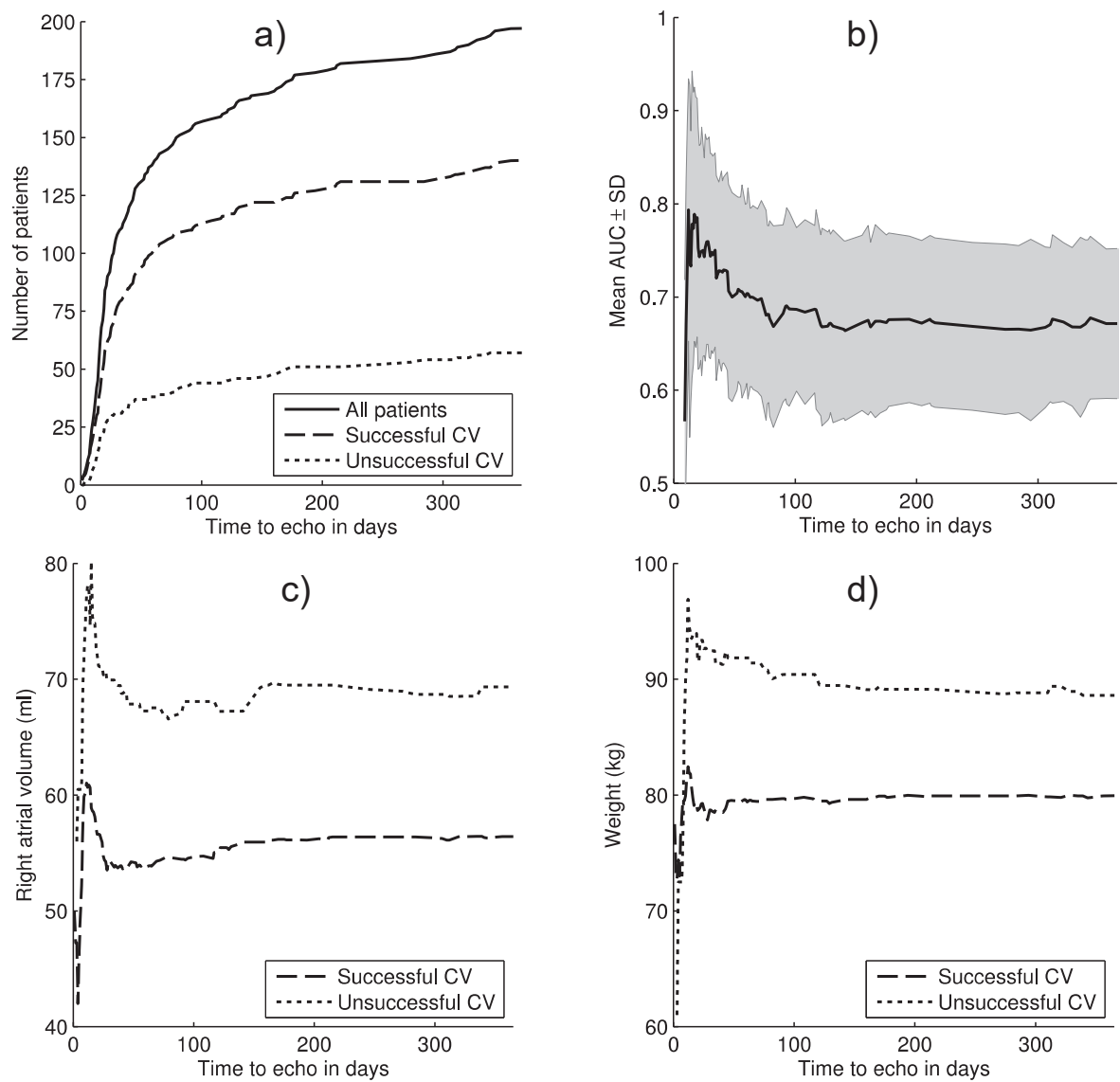

Figure 3: The effect of maximum allowed time difference between the date of cardioversion (CV) and the closest date of an echocardiography on (a) the number of patients included in the analysis, (b) prediction performance of the best performing model containing clinical parameters weight and right atrial volume, (c) differences in patient right atrial volume (successful and unsuccessful CV), and (d) differences in patient weight. 
Predicting pharmacological cardioversion

\section{Results}

\section{Effect of time interval between echocardiography and CV attempt}

In our analysis we included echocardiographic data that was collected within a year (365 days) of the date of the CV attempt. In this analysis we also included patients without an ECG or a poor quality ECG before the CV attempt $(n=198)$. Results are shown in Figure 3. From Figure 3a it becomes clear that the number of patients that can be included in the analysis based on their echocardiographic data, initially decreases slowly when we move from 365 days to a narrower timeframe. This decrease accelerates when we reach 100 days as a cut-off value. The performance of the best model containing only clinical parameters (weight and right atrial volume (RAV)), shown in Figure $3 b$, remains relatively stable until 100 days, and then starts to increase, but also becomes more irregular, due to the lower number of patients included in the analysis. This observation is supported by examining the evolution of the two clinical parameters forming the best performing model, as shown in Figure $3 \mathrm{c}$ and $3 d$. 
Chapter 4

\begin{tabular}{|c|c|c|c|c|c|}
\hline Parameter & Lead & $\begin{array}{c}\text { Successful } \\
\text { PCV }\end{array}$ & $\begin{array}{c}\text { Unsuccessful } \\
\text { PCV }\end{array}$ & P-value & AUC \\
\hline \multicolumn{6}{|c|}{ Frequency domain } \\
\hline $\mathrm{DF}(\mathrm{Hz})$ & II & $5.9(1.0)$ & $6.3(1.1)$ & $<0.001$ & 0.66 \\
\hline \multirow[t]{4}{*}{ Welch } & III & $6.1(0.7)$ & $6.3(1.2)$ & 0.009 & 0.60 \\
\hline & $\mathrm{aVR}$ & $5.9(1.2)$ & $6.3(1.2)$ & 0.008 & 0.61 \\
\hline & aVF & $5.9(1.0)$ & $6.3(1.5)$ & 0.007 & 0.60 \\
\hline & $\mathrm{V}_{1}$ & $6.3(1.2)$ & $6.6(1.6)$ & 0.027 & 0.59 \\
\hline RHE & I & $0.201(0.155)$ & $0.166(0.106)$ & 0.043 & 0.58 \\
\hline OI (\%) & III & $57.8 \pm 17.7$ & $53.1 \pm 19.7$ & 0.020 & 0.59 \\
\hline SE & $\mathrm{V}_{6}$ & $5.67(0.67)$ & $5.83(0.55)$ & 0.043 & 0.58 \\
\hline \multicolumn{6}{|c|}{ Time domain } \\
\hline \multirow[t]{2}{*}{ SAE } & II & $0.317 \pm 0.046$ & $0.341 \pm 0.055$ & 0.001 & 0.63 \\
\hline & aVF & $0.323 \pm 0.060$ & $0.345 \pm 0.061$ & 0.010 & 0.60 \\
\hline FWP MAW & $\mathrm{aVL}$ & $0.0064 \pm 0.0018$ & $0.0058 \pm 0.0018$ & 0.021 & 0.59 \\
\hline \multirow[t]{5}{*}{ FWA (mV) } & II & $0.055(0.024)$ & $0.051(0.017)$ & 0.044 & 0.58 \\
\hline & III & $0.059(0.024)$ & $0.053(0.021)$ & 0.007 & 0.61 \\
\hline & $\mathrm{aVL}$ & $0.044(0.016)$ & $0.040(0.015)$ & 0.023 & 0.58 \\
\hline & $\mathrm{aVF}$ & $0.053(0.024)$ & $0.048(0.016)$ & 0.016 & 0.60 \\
\hline & $\mathrm{V}_{6}$ & $0.038(0.012)$ & $0.034(0.010)$ & 0.018 & 0.59 \\
\hline
\end{tabular}

Table 4: Significant single lead parameter differences and prediction AUC. DF: Dominant Frequency, FWA: Fibrillation Wave Amplitude, FWP MAW: Fibrillation Wave Power of the Main Atrial Wave, OI: Organization Index, PCV: pharmacological cardioversion, RHE: Relative Harmonic Energy, SAE: Sample Entropy, SE: Spectral Entropy. 


\begin{tabular}{|c|c|c|c|c|c|}
\hline Parameter & $\begin{array}{l}\text { Leads or } \\
\text { Signal }\end{array}$ & $\begin{array}{l}\text { Successful } \\
\text { PCV }\end{array}$ & $\begin{array}{l}\text { Unsuccessful } \\
\text { PCV }\end{array}$ & P-value & AUC \\
\hline \multicolumn{6}{|c|}{ Frequency domain } \\
\hline MDF (Hz) & $\mathrm{V}_{(2,5)}$ & $6.0(1.0)$ & $6.3(1.3)$ & 0.008 & 0.61 \\
\hline Top 4 & $V_{(1,4,5)}$ & $6.0(1.3)$ & $6.8(1.5)$ & 0.003 & 0.62 \\
\hline \multirow[t]{2}{*}{ (of 42) } & $V_{(1,2,4,6)}$ & $6.0(1.3)$ & $6.5(1.4)$ & 0.001 & 0.63 \\
\hline & $V_{(1,2,4,5,6)}$ & $6.0(1.3)$ & $6.5(1.3)$ & 0.001 & 0.64 \\
\hline MOI (\%) & $V_{(3,4)}$ & $50.6 \pm 8.7$ & $47.6 \pm 6.9$ & 0.015 & 0.60 \\
\hline Top 3 & $V_{(1,2,4)}$ & $53.8(10.2)$ & $51.2(10.0)$ & 0.005 & 0.61 \\
\hline (of 22) & $V_{(2,4,5,6)}$ & $41.6(8.0)$ & $38.9(6.2)$ & 0.005 & 0.61 \\
\hline MSE & $V_{(3,4)}$ & $6.37 \pm 0.39$ & $6.49(0.34)$ & 0.046 & 0.59 \\
\hline SC (\%) & All leads & $23.8(1.4)$ & $23.5(1.5)$ & 0.031 & 0.58 \\
\hline SV & All leads & $0.51(0.26)$ & $0.66(0.37)$ & 0.001 & 0.64 \\
\hline \multicolumn{6}{|c|}{ Time domain } \\
\hline \multirow[t]{2}{*}{$\mathbf{k}_{0.95}$} & $\mathrm{AA}$ & $4.8(0.8)$ & $5.0(0.8)$ & 0.033 & 0.58 \\
\hline & TQ & $3.2(0.6)$ & $3.4(0.4)$ & 0.050 & 0.57 \\
\hline MFWA & AA & $0.049(0.037)$ & $0.040(0.031)$ & 0.025 & 0.59 \\
\hline \multirow[t]{2}{*}{ C } & $\mathrm{AA}$ & $9.4(3.5)$ & $10.7(4.3)$ & 0.021 & 0.59 \\
\hline & TQ & $4.6 \pm 1.8$ & $5.2 \pm 2.0$ & 0.050 & 0.57 \\
\hline \multirow[t]{2}{*}{ CV } & AA & $2.5 \pm 1.0$ & $3.0 \pm 1.5$ & 0.005 & 0.58 \\
\hline & $\mathrm{TQ}$ & $2.8 \pm 1.2$ & $3.2 \pm 1.3$ & 0.076 & 0.57 \\
\hline
\end{tabular}

Table 5: Significant multidimensional parameter differences and prediction AUC. C: spatial complexity parameters, CV: Variability of spatial complexity, DF: Dominant Frequency, MDF/MOI/MSE: Multidimensional DF/OI/SE, OI: Organization Index, PCV: Pharmacological cardioversion, SC: Spectral Concentration, SE: Spectral Entropy, SV: Spectral Variability, MFWA: Multidimensional Fibrillation Wave Amplitude. 


\section{Chapter 4}

\section{References}

1. Bonizzi $P$, Meste $O$, Zarzoso V: Spectral analysis of atrial signals directly from surface ECG exploiting compressed spectrum. Computers in Cardiology, 2008 IEEE, 2008; :221-224.

2. Stoffer DS, Tyler DE, Wendt DA: The spectral envelope and its applications. Statistical Science JSTOR, 2000; :224-253.

3. Uldry L, Van Zaen J, Prudat Y, Kappenberger L, Vesin J-M: Measures of spatiotemporal organization differentiate persistent from long-standing atrial fibrillation. Europace 2012; 14:1125-1131.

4. Nault I, Lellouche N, Matsuo S, et al.: Clinical value of fibrillatory wave amplitude on surface ECG in patients with persistent atrial fibrillation. J Interv Card Electrophysiol 2009; 26:11-19.

5. Meo M, Zarzoso V, Meste O, Latcu DG, Saoudi N: Spatial variability of the 12-lead surface ECG as a tool for noninvasive prediction of catheter ablation outcome in persistent atrial fibrillation. IEEE Trans Biomed Eng 2013; 60:20-27.

6. Alcaraz R, Sandberg F, Sörnmo L, Rieta JJ: Classification of paroxysmal and persistent atrial fibrillation in ambulatory ECG recordings. IEEE Trans Biomed Eng 2011; 58:1441-1449.

7. Bonizzi P, Guillem M de LS, Climent AM, Millet J, Zarzoso V, Castells F, Meste O: Noninvasive assessment of the complexity and stationarity of the atrial wavefront patterns during atrial fibrillation. IEEE Trans Biomed Eng 2010; 57:2147-2157.

8. Di Marco LY, Bourke JP, Langley P: Spatial complexity and spectral distribution variability of atrial activity in surface ECG recordings of atrial fibrillation. Med Biol Eng Comput 2012; 50:439-446.

9. Friedman J, Hastie T, Tibshirani R: Regularization Paths for Generalized Linear Models via Coordinate Descent. J Stat Softw 2010; 33:1-22. 



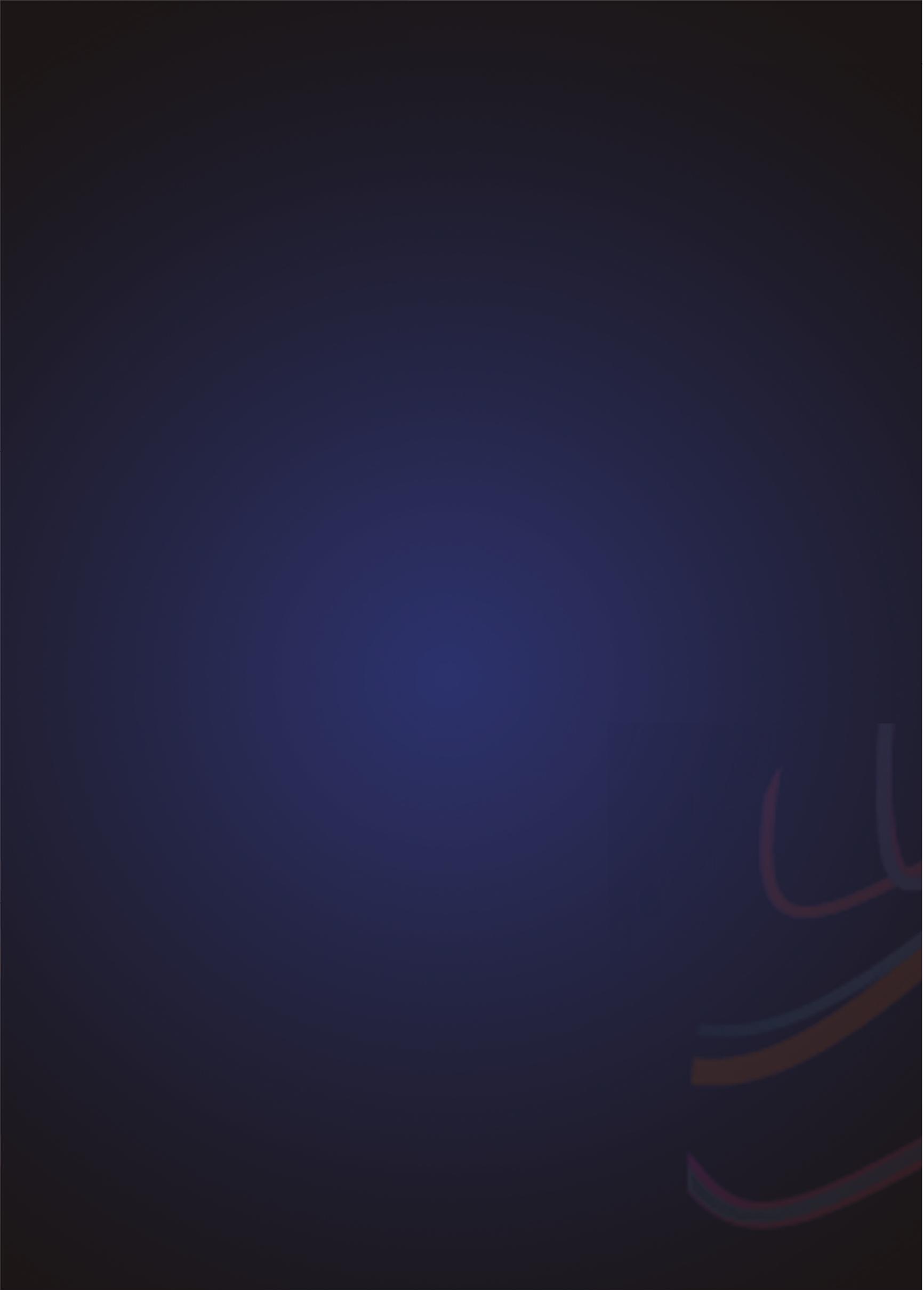




\section{Chapter 5}

Systematic analysis of ECG

predictors for sinus rhythm

maintenance following electrical

cardioversion

Theo Lankveld, Cees B de Vos, Ione Limantoro, Stef

Zeemering, Elton Dudink, Harry J Crijns and Ulrich Schotten

Heart Rhythm 2016;13:1020-7 
Chapter 5

\section{Abstract}

Background: Electrical cardioversion (ECV) is one of the rhythm control strategies in patients with persistent atrial fibrillation (AF). Unfortunately, recurrences of AF are very common after ECV which significantly limits the practical benefit of this treatment in AF patients.

Objective: The objective of this study was to identify non-invasive complexity or frequency parameters obtained from the surface electrocardiogram (ECG) to predict sinus rhythm (SR) maintenance after ECV and to compare these ECG parameters with clinical predictors.

Methods: We studied a large variety of ECG-derived time and frequency domain AF complexity parameters in a prospective cohort of 502 patients with persistent AF referred for ECV.

Results: During 1 year follow-up 161 patients (32\%) maintained SR. The best clinical predictor for SR maintenance was anti-arrhythmic drug (AAD) treatment. A model including clinical parameters predicted SR maintenance with a mean cross-validated receiver operator area under the curve (AUC) of $0.62 \pm 0.05$. The best single ECG parameter was the dominant frequency (DF) on lead V6. Combining several ECG parameters predicted SR maintenance with a mean AUC $0.64 \pm 0.06$. Combining clinical and ECG parameters improved prediction to a mean AUC $0.67 \pm 0.05$. Although the DF was influenced by AAD treatment, excluding patients on AADs did not significantly lower the predictive performance captured by the ECG.

Conclusions: ECG derived parameters predict SR maintenance during 1 year follow-up after ECV at least as good as known clinical predictors for rhythm outcome. The DF proved to be the most powerful ECG-derived predictor.

Keywords: Atrial fibrillation, Electrocardiography, Electrical cardioversion 


\section{Introduction}

In patients with atrial fibrillation (AF), both a rate and a rhythm control strategy can be the treatment of choice. ${ }^{1}$ Although both strategies result in comparable mortality and morbidity in patients with symptomatic AF, rhythm control is preferred. ${ }^{1,2}$ Therapeutic strategies to obtain rhythm control include electrical cardioversion (ECV), catheter or surgical ablation, both with or without the support of anti-arrhythmic drugs (AADs). In patients with persistent $A F, E C V$ is frequently used to acutely restore sinus rhythm (SR) because of its high efficacy and low complication rates. However, long-term success of ECV in patients with persistent AF is limited. ${ }^{3}$ The success rates of ECV can be improved by AADs but at the cost of potential side effects. ${ }^{4,5}$ Considering the above, it would be desirable to identify - before ECV - patients with a high likelihood of maintaining $S R$ and conversely those in whom AF is likely to recur. In patients with a high likelihood of SR maintenance after ECV, this method may be the treatment of choice. In patients with a low likelihood of success, either ECV supported by AADs, ablation, or a rate-control strategy could be more appropriate treatment options.

Several clinical and echocardiographic parameters have been used to identify patients likely to maintain SR. ${ }^{6,} 7$ In addition to clinical history, an electrocardiogram (ECG) is generally easily available in every patient with persistent AF. Therefore, several studies used the ECG to predict success of ECV. A wide variety of non-invasive ECG-derived parameters have been suggested. ${ }^{8}$ However, studies examining noninvasive ECG parameters show variable predictive performances. ${ }^{9-11}$ Table 1 provides an overview of previous studies on the predictive value of ECG parameters for outcome prediction in clinical studies with patients undergoing ECV. The results of the studies addressing the predictive value of the dominant frequency (DF) of the atrial component of the ECG vary considerably. Only 1 study with short follow-up evaluated the predictive value of the F-wave amplitude (FWA). Most studies were relatively small (varying from 37 to 175 patients), and many parameters showing predictive value in other clinical settings have not been studied in patients undergoing ECV. This emphasizes the need for a systematic comparison of these parameters in evaluating their predictive performance. ${ }^{12,13}$

We systematically assessed the predictive value of a wide variety of established and new ECG-derived parameters for SR maintenance after ECV on all 12 ECG leads in a large cohort of patients with persistent AF. We tested the hypothesis that ECG-derived frequency and complexity parameters can predict recurrences of $A F$ after ECV at least as good as current clinical predictors. 
Chapter 5

\begin{tabular}{|c|c|c|c|}
\hline Parameter & $\begin{array}{l}\text { Parameter } \\
\text { domain }\end{array}$ & $\begin{array}{l}\text { Parameter } \\
\text { description }\end{array}$ & Main results \\
\hline $\begin{array}{l}\text { Dominant } \\
\text { frequency }\end{array}$ & Frequency & $\begin{array}{l}\text { Frequency with the } \\
\text { strongest power in } \\
\text { the power spectrum. }\end{array}$ & $\begin{array}{l}\text { A low DF predicts SR } \\
\text { maintenance after ECV in } \\
\text { most but not all studies. }\end{array}$ \\
\hline $\begin{array}{l}\text { Organization } \\
\text { index }\end{array}$ & Frequency & $\begin{array}{l}\text { Ratio of the two } \\
\text { peaks with the } \\
\text { strongest power in } \\
\text { the power spectrum } \\
\text { to the entire power } \\
\text { spectrum. }\end{array}$ & $\begin{array}{l}\text { Not previously studied in } \\
\text { patients undergoing ECV. }\end{array}$ \\
\hline $\begin{array}{l}\text { Spectral } \\
\text { entropy }\end{array}$ & Frequency & $\begin{array}{l}\text { Spectral entropy can } \\
\text { be considered a } \\
\text { measure of } \\
\text { uniformity of the } \\
\text { spectral content. }\end{array}$ & $\begin{array}{l}\text { Not previously studied in } \\
\text { patients undergoing ECV. }\end{array}$ \\
\hline $\begin{array}{l}\text { Sample } \\
\text { entropy }\end{array}$ & Time & $\begin{array}{l}\text { Sample entropy is an } \\
\text { estimate of the } \\
\text { regularity within the } \\
\text { signal. }\end{array}$ & $\begin{array}{l}\text { A lower sample entropy } \\
\text { predicts SR maintenance } \\
\text { after ECV during short- } \\
\text { term follow-up. }\end{array}$ \\
\hline $\begin{array}{l}\text { F-wave } \\
\text { amplitude }\end{array}$ & Time & $\begin{array}{l}\text { Mean amplitude of } \\
\text { the F-waves }\end{array}$ & $\begin{array}{l}\text { A higher FWA predicts SR } \\
\text { maintenance in patients } \\
\text { undergoing ECV during } \\
\text { short-term follow-up. }\end{array}$ \\
\hline
\end{tabular}

Table 1: Overview of previous studies using ECG parameters to predict ECV outcome. 


\section{Methods}

We prospectively included patients with persistent AF who underwent ECV at the Maastricht University Medical Centre, Maastricht, the Netherlands. Persistent AF was defined as AF on at least 2 consecutive ECGs or Holterrecordings, at least 7 days apart. A digital ECG was recorded during AF at the day of cardioversion and patients were followed for 1 year. Patients needed to be on adequate anticoagulation for at least 4 weeks before ECV. Patients younger than 18 years, with atrial flutter at the time of ECV or hemodynamically unstable patients were excluded. The study was approved by the Institutional ethics review board.
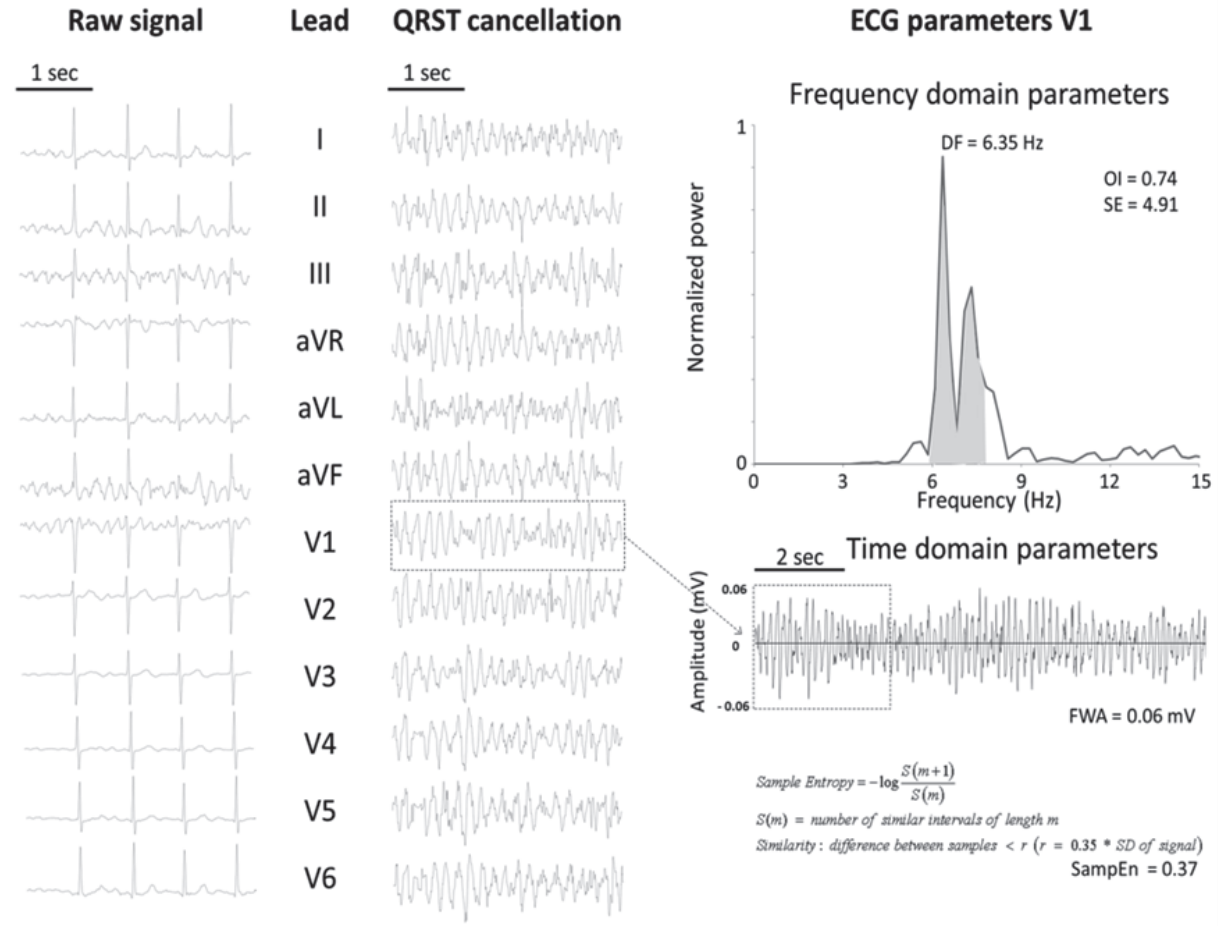

Figure 1: This figure shows the computation of the ECG-derived parameters. The left panel shows 3 seconds of every lead before any filtering or pre-processing steps. The middle panel shows the same 3 seconds after $1-100 \mathrm{~Hz}$ bandpass filtering and QRST cancellation. The right panel shows the ECG-derived parameters computed on lead V1. The upper right panel shows the frequency-domain parameters computed on the frequency power spectrum: dominant frequency (DF), organization index (OI), spectral entropy (SE). The bottom right panel shows the time-domain parameters: F-wave amplitude (FWA) and sample entropy (SampEn). 


\section{End point and follow-up}

The primary end point was AF recurrence. AF recurrence was defined as either unsuccessful ECV or as documented AF on a Holter or 12-lead ECG after initial successful ECV. Patients with SR maintenance had no documented AF recurrence within 1 year of ECV. Early recurrence was defined as a documented AF recurrence within 4 weeks or asymptomatic AF recurrence at the first visit. Routine control visits were conducted by the referring physician 1 month after ECV and thereafter as indicated necessary. During each followup visit, an ECG was recorded and symptoms were evaluated. If patients suffered from cardiac complaints, an ECG was recorded in the emergency department.

\section{ECG analyses}

A 10-second ECG was digitally recorded with a sampling frequency of $250 \mathrm{~Hz}$ at the day of ECV. The ECG was imported in custom-made Matlab software (MATLAB and Statistics Toolbox Release 2014a, The MathWorks, Inc., Natick, Massachusetts, United States) for the analysis. ECGs were filtered using a zerophase band-pass filter between 1 and $100 \mathrm{~Hz}$ to remove baseline wandering and supress high-frequency noise. Power line interference, if present, was removed using a $50 \mathrm{~Hz}$ notch filter. Ventricular signals were cancelled using a modified version of the adaptive singular value QRST cancellation. This method groups QRST windows before QRST template computation by using hierarchical clustering based on window correlation. ${ }^{14}$ Before the analyses, an additional $3 \mathrm{~Hz}$ high-pass filter was used to avoid interference caused by the (possible residual) T-wave.

A variety of parameters were calculated to cover both the time and frequency domain. For frequency parameters, a frequency power spectrum was estimated using Welch's power spectral density estimate. DF was defined as the frequency with the highest power between 3 and $12 \mathrm{~Hz}$ in this frequency spectrum. For the organization index, the areas of the two highest peaks of the frequency spectrum were calculated using a $1 \mathrm{~Hz}$ frequency interval centred on each peak. The organization index was defined as the ratio of the cumulative areas of these two peaks to the area of the entire power spectrum. To compute the spectral entropy, the spectrum was first normalized in order to produce a probability mass function, on which Shannon entropy was calculated to give an estimate of spectral entropy. For the FWA, the mean amplitude of the F-waves on the extracted atrial signal was calculated, with a minimum distance between two peaks of 100ms. For sample entropy (SampEn) computation, the number of samples used was 3 within a tolerance of $r=0.35$ 
times the standard deviation. Figure 1 shows an overview of the complexity and frequency parameters computed in a patient.

\section{Clinical and echocardiographic parameters}

Detailed medical history and drug treatment were collected at the day of ECV. Echocardiography was performed to evaluate left ventricular function, and left and right atrial dimensions during AF.

\section{Statistical analysis}

Statistical analysis was performed using SPSS statistical software (IBM SPSS statistics version 21.0). Categorical variables are reported as number of patients and percentage. Continuous variables are presented as mean \pm SD or as median with interquartile range depending on their normal distribution. Differences between continuous variables were compared using the Student's t-test or the Mann-Whitney $U$ test in not normally distributed data. For the models we used a forward logistic regression analysis using either the clinical parameters, ECG-parameters or the combination of those parameters. We used 5 -fold cross-validation for internal validation of the models. A P-value of 0.05 was considered statistically significant.

\section{Results}

\section{Population}

The ECGs of 502 patients were suitable for the analyses. SR was maintained in 161 patients (32\%) during the first year after ECV. ECV failed to restore SR in 50 patients (10\%); another 196 patients (39\%) experienced an early recurrence; and the remaining 95 patients (19\%) experienced a late recurrence. Baseline characteristics are listed in table 2 . The appendix provides an detailed overview of the patients' clinical characteristics per recurrence category. 


\begin{tabular}{|c|c|c|c|c|}
\hline & $\begin{array}{l}\text { Overall } \\
\mathrm{N}=502\end{array}$ & $\begin{array}{c}\text { SR maintenance } \\
\qquad \mathrm{N}=161\end{array}$ & $\begin{array}{l}\text { AF recurrence } \\
\qquad \mathrm{N}=\mathbf{3 4 1}\end{array}$ & $P$ value \\
\hline Age (years) & $66 \pm 10$ & $67 \pm 12$ & $66 \pm 9$ & 0.443 \\
\hline Male & $354(71 \%)$ & $116(72 \%)$ & $238(70 \%)$ & 0.605 \\
\hline BMI & $28.8 \pm 5.1$ & $28.6 \pm 5.0$ & $29.0 \pm 5.1$ & 0.490 \\
\hline Hypertension & 302 (60\%) & $99(62 \%)$ & $203(60 \%)$ & 0.675 \\
\hline Diabetes & $57(11 \%)$ & $16(10 \%)$ & $41(12 \%)$ & 0.492 \\
\hline CAD & $134(27 \%)$ & $43(27 \%)$ & $91(27 \%)$ & 0.996 \\
\hline PM/ICD & $29(6 \%)$ & $8(5 \%)$ & $21(6 \%)$ & 0.594 \\
\hline Heart Failure & $131(26 \%)$ & $43(27 \%)$ & $88(26 \%)$ & 0.830 \\
\hline Stroke & $49(10 \%)$ & $10(6 \%)$ & $39(11 \%)$ & 0.066 \\
\hline COPD & $49(10 \%)$ & $10(6 \%)$ & $39(11 \%)$ & 0.066 \\
\hline OSAS & $31(6 \%)$ & $8(5 \%)$ & $23(7 \%)$ & 0.440 \\
\hline $\mathrm{CHA}_{2} \mathrm{DS}_{2}$-VASc & $2.6 \pm 1.6$ & $2.6 \pm 1.4$ & $2.6 \pm 1.6$ & 0.977 \\
\hline $\begin{array}{l}\text { Total AF duration } \\
\text { (Months) }\end{array}$ & $7[3-45]$ & $7[3-44]$ & $7[3-46]$ & 0.989 \\
\hline AF episode (Months) & $3[2-5]$ & $3[1-5]$ & $3[2-6]$ & 0.112 \\
\hline Previous ECV & $138(28 \%)$ & $39(24 \%)$ & 99 (29\%) & 0.260 \\
\hline \multicolumn{5}{|l|}{ Medication } \\
\hline Diuretic & $233(46 \%)$ & $80(50 \%)$ & $153(45 \%)$ & 0.312 \\
\hline ACE or ARB & 348 (69\%) & $112(70 \%)$ & 236 (69\%) & 0.935 \\
\hline Beta-blocker & 393 (78\%) & $132(82 \%)$ & $261(77 \%)$ & 0.167 \\
\hline Digoxin & $139(28 \%)$ & $44(27 \%)$ & $95(28 \%)$ & 0.901 \\
\hline Statin & 249 (50\%) & $88(55 \%)$ & $161(47 \%)$ & 0.119 \\
\hline Any AAD & $132(26 \%)$ & $59(37 \%)$ & $73(21 \%)$ & $<0.001$ \\
\hline Amiodarone & $80(16 \%)$ & $39(24 \%)$ & $41(12 \%)$ & $<0.001$ \\
\hline Flecainide & $11(2 \%)$ & $5(3 \%)$ & $6(2 \%)$ & 0.336 \\
\hline Sotalol & $42(8 \%)$ & $16(10 \%)$ & $26(8 \%)$ & 0.382 \\
\hline \multicolumn{5}{|l|}{ Echocardiogram } \\
\hline LVEF (\%) & $50 \pm 12$ & $50 \pm 13$ & $50 \pm 12$ & 0.945 \\
\hline LAV (mL) & $96 \pm 31$ & $95 \pm 30$ & $96 \pm 32$ & 0.790 \\
\hline $\operatorname{RAV}(\mathrm{mL})$ & $81 \pm 30$ & $77 \pm 27$ & $83 \pm 32$ & 0.032 \\
\hline
\end{tabular}

Table 2: Baseline characteristics. Anti-arrhythmic drug (AAD), angiotensin receptor blocker (ARB) Atrial fibrillation (AF), body mass index (BMI), coronary artery disease (CAD), chronic obstructive pulmonary disease (COPD), electrical cardioversion (ECV), implantable cardiac defibrillator (ICD), left atrial volume (LAV), left ventricular ejection fraction (LVEF), pacemaker (PM), right atrial volume (RAV), sinus rhythm (SR). 


\section{Predictors of maintenance of sinus rhythm}

A significantly higher proportion of patients on AADs remained in SR during follow-up than patients without AADs (table 2) $(P<0.001)$. Especially patients taking amiodarone maintained SR during follow-up. The clinical prediction model included the use of any AAD, $\beta$-blocker use and a previous ECV for persistent AF. The model including these clinical parameters predicted maintenance of SR with a mean area under the curve (AUC) of $0.62 \pm 0.05$ (figure 2). The DF was the strongest univariate ECG predictor. The AF-free survival curves for every parameter on the best performing lead showed the highest odds ratio (OR) for the DF on lead V6 (OR 2.25 (1.53 - 3.29)) (figure 3). Table 3 reveals that patients who remained in SR after one year of follow-up had a significantly lower DF than did those with an AF recurrence $(P<0.001$ on most leads). Dispersion of DF across leads defined as a difference of $0.5 \mathrm{~Hz}$ between any two leads showed no significant differences between the groups. The same holds true for a difference of $1.0 \mathrm{~Hz}$ or more. Interestingly, the DF was not significantly higher in patients with an early AF recurrence or ECV failure compared to patients without an early recurrence on most of the leads (table 3). Combining ECG parameters provided a model with a mean AUC of $0.64 \pm 0.06$ including the DF on lead V6 and SampEn on lead V5. The optimal threshold derived from the receiver operating characteristic (ROC) curve, with a sensitivity of $72 \%$ and a specificity of $49 \%$, predicted AF recurrence after 1 year follow-up with an OR of $2.51(1.70-3.70 ; \mathrm{P}<0.001)$ (figure 4). Using the same threshold, the model predicted early recurrences less accurately (OR 1.60; $95 \% \mathrm{Cl} 1.10-2.32$ ). Combining clinical and ECG parameters improved prediction slightly to a mean AUC of $0.67 \pm 0.06$ (figure 2). The optimal threshold of this model had a sensitivity of $78 \%$ and a specificity of $48 \%$. This threshold predicted AF recurrence after 1-year follow-up with an OR $3.73(95 \%$ Cl 2.49-5.60; $\mathrm{P}<0.001$ ). 


\begin{tabular}{lcccccc}
\hline & $\begin{array}{c}\text { No early } \\
\text { recurrence } \\
\mathbf{N}=\mathbf{2 5 5}\end{array}$ & $\begin{array}{c}\text { Early AF } \\
\text { recurrence } \\
\text { or ECV failure } \\
\mathbf{N = 2 4 7}\end{array}$ & $\begin{array}{c}\mathbf{P} \\
\text { value }\end{array}$ & $\begin{array}{c}\text { SR } \\
\text { maintenance } \\
\mathbf{1} \text { year } \\
\mathbf{N = 1 6 1}\end{array}$ & $\begin{array}{c}\text { AF } \\
\text { recurrence } \\
\mathbf{1} \text { year } \\
\mathbf{N = 3 4 1}\end{array}$ & P value \\
\hline Lead & & & & & & \\
I & $6.34 \pm 1.02$ & $6.52 \pm 0.81$ & 0.029 & $6.16 \pm 1.01$ & $6.55 \pm 0.86$ & $<0.001$ \\
II & $6.23 \pm 0.97$ & $6.38 \pm 0.71$ & 0.046 & $6.08 \pm 0.97$ & $6.41 \pm 0.77$ & $<0.001$ \\
III & $6.25 \pm 1.00$ & $6.46 \pm 0.82$ & 0.012 & $6.08 \pm 0.95$ & $6.49 \pm 0.88$ & $<0.001$ \\
aVR & $6.27 \pm 1.00$ & $6.41 \pm 0.74$ & 0.088 & $6.11 \pm 1.03$ & $6.45 \pm 0.78$ & $<0.001$ \\
aVL & $6.30 \pm 1.02$ & $6.48 \pm 0.74$ & 0.033 & $6.12 \pm 1.00$ & $6.52 \pm 0.86$ & $<0.001$ \\
aVF & $6.27 \pm 1.02$ & $6.42 \pm 0.72$ & 0.066 & $6.09 \pm 1.00$ & $6.47 \pm 0.80$ & $<0.001$ \\
V1 & $6.44 \pm 1.03$ & $6.54 \pm 0.76$ & 0.188 & $6.27 \pm 1.03$ & $6.59 \pm 0.83$ & 0.001 \\
V2 & $6.38 \pm 1.07$ & $6.51 \pm 0.81$ & 0.149 & $6.26 \pm 1.11$ & $6.53 \pm 0.85$ & 0.007 \\
V3 & $6.35 \pm 0.94$ & $6.41 \pm 0.76$ & 0.406 & $6.20 \pm 0.95$ & $6.47 \pm 0.79$ & 0.002 \\
V4 & $6.33 \pm 0.93$ & $6.42 \pm 0.79$ & 0.252 & $6.16 \pm 0.93$ & $6.48 \pm 0.82$ & $<0.001$ \\
V5 & $6.32 \pm 0.97$ & $6.43 \pm 0.77$ & 0.157 & $6.12 \pm 0.96$ & $6.49 \pm 0.81$ & $<0.001$ \\
V6 & $6.32 \pm 1.02$ & $6.48 \pm 0.78$ & 0.059 & $6.10 \pm 0.98$ & $6.54 \pm 0.84$ & $<0.001$ \\
\hline
\end{tabular}

Table 3: Dominant frequency per lead. Atrial fibrillation (AF), electrical cardioversion (ECV), sinus rhythm (SR).

\section{Anti-arrhythmic drug treatment}

The DF was significantly lower on all leads in patients taking AADs $(P<0.001)$. AAD use was also associated with a lower SampEn $(P<0.001)$ but weaker or no association of AAD use was found with other complexity parameters. In 370 patients without AADs, the right atrial volume was significantly smaller and the AF episode significantly shorter in patients who remained in SR. Furthermore, patients with an $A F$ recurrence less frequently used $\beta$-blockers and more often received a previous ECV for persistent AF. DF remained the strongest ECG predictor for SR maintenance. The predictive performance of the models decreased only slightly with a mean AUC of $0.65 \pm 0.06$ for the combined model. The main difference was the introduction of the FWA on lead aVR instead of SampEn.

\section{Reproducibility}

In 98 patients, a second digitally recorded ECG was available in the month before ECV. We found a moderate to fair intraclass correlation coefficient (ICC) of 0.652 ( $95 \% \mathrm{Cl} 0.481-0.767$; $\mathrm{P}<0.001$ ) for lead V6. The ICC improves by limiting time between ECGs. In 48 patients with 2 ECGs, within 14 days the ICC was $0.739(95 \% \mathrm{Cl} 0.528-0.855)$. 

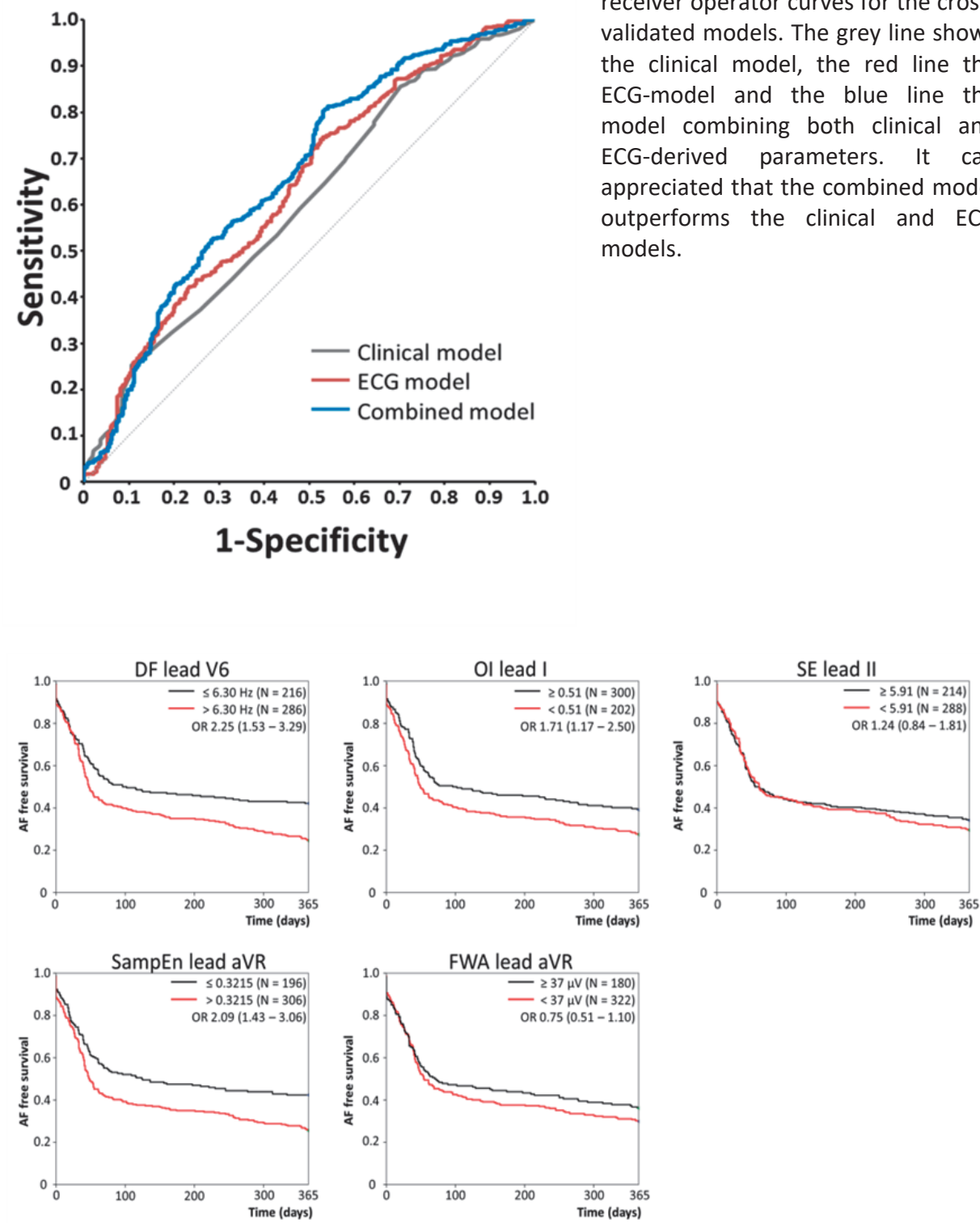

Figure 2: This figure shows the receiver operator curves for the crossvalidated models. The grey line shows the clinical model, the red line the ECG-model and the blue line the model combining both clinical and ECG-derived parameters. It can appreciated that the combined model outperforms the clinical and ECG models.

- Clinical model

ECG model

Combined model

$\begin{array}{lllll}6 & 0.7 & 0.8 & 0.9 & 1.0\end{array}$

. 

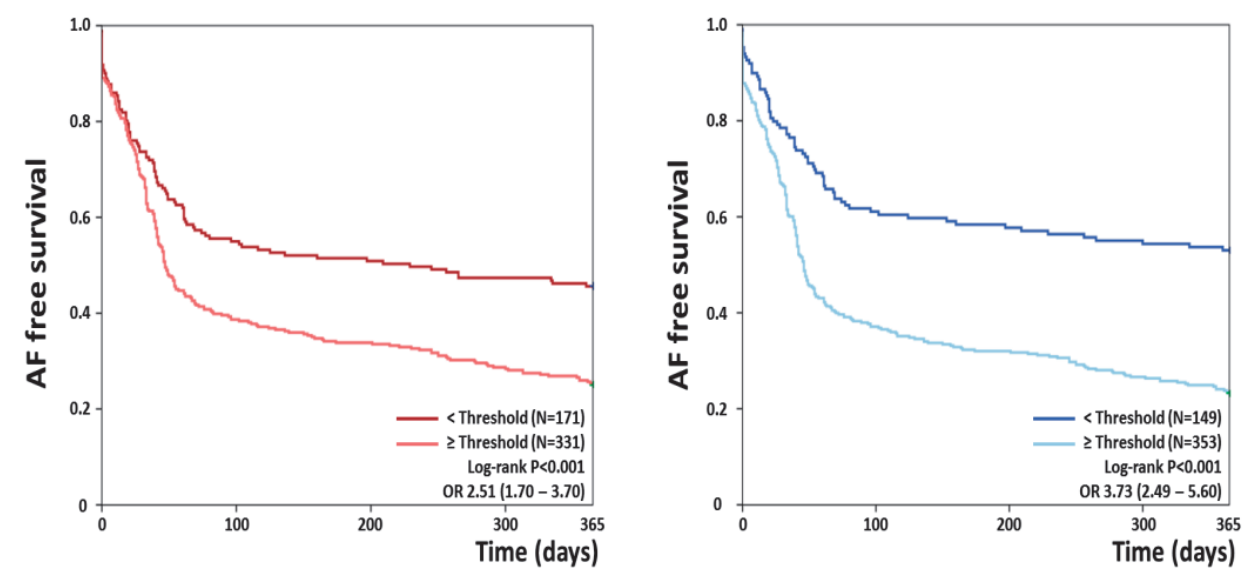

Figure 4: This figure shows the AF-free survival curves for the optimal cut-off derived from the ROC-curves. In red the optimal cut-off for the ECG model and in blue the optimal cut-off for the combined model is shown.

\section{Discussion}

This is the largest and first systematic comparative study investigating a wide variety ECG-derived frequency and complexity parameters on all 12 leads for the prediction of long-term maintenance of SR after ECV. The study confirms the capability of these parameters to predict SR maintenance after ECV. Because of the large patient population and internal validation process used, we believe that these findings are robust and transferable to other populations in the setting of ECV of persistent AF. A lower DF was the strongest ECG parameter to predict patients likely to maintain SR. A model including ECGderived parameters outperformed known clinical predictors for SR maintenance after ECV. Combining the 2 further increased the predictive performance.

\section{DF predicting SR maintenance after ECV}

The DF was the most promising ECG-derived parameter. As in our study, most previous studies that investigated the predictive value of the DF for SR maintenance after ECV showed the favourable rhythm outcome in patients with a lower DF. These results, however, were not consistent in all studies, which might partially be due to the fact that the duration of the follow-up period varied markedly between studies. ${ }^{10,11,15-18}$ Our results demonstrate that the noninvasively measured DF was able to identify patients likely to be in SR after 1 year. In contrast, this model could predict early SR maintenance less accurately. The difference between the predictive values for early and late 
recurrences might be due to different pathophysiological mechanisms causing these recurrences. ${ }^{19}$ Early AF recurrences are mainly due to a high incidence of triggers such as (early coupled) atrial premature beats shortly after ECV. ${ }^{20}$ The DF measured on the ECG does not reflect these triggers and is therefore not likely to identify patients with a recurrence due to these frequently occurring triggers. In the later phase after ECV, atrial electroanatomical remodeling plays a more important role in the recurrence and maintenance of AF. These remodeling processes are obviously better reflected by the frequency and complexity parameters measured on the ECG. Triggers such as atrial ectopic beats can be suppressed using AADs. The combined model (including AAD treatment) performed better in predicting early recurrences (OR $2.30 ; 95 \% \mathrm{Cl}$ $1.55-3.42$ for the optimal cut-off), which supports the hypothesis that these triggers are responsible for early recurrences. Furthermore, it might be worthwhile to do a post hoc analysis in patients with a low DF in trials that used AADs to prevent (early) recurrences such as FLEC-SL and CONVERT trials. ${ }^{21,} 22$ An advantage of the DF over more complicated ECG-derived complexity parameters is that it is easy to calculate and can already be determined using additional software packages in some ECG recording systems. ${ }^{23}$ As expected, the reproducibility becomes less with increasing time between the ECGs possibly by an advanced remodeling process which is known to effect the atrial frequency.

\section{Other ECG parameters for SR maintenance}

Although a low DF was the best ECG predictor for SR maintenance during follow-up, 2 other parameters seemed to have additional value. A lower SampEn or higher FWA were predictive of SR maintenance, depending on the patients included in the analysis (with or without AADs, respectively). A lower SampEn has been shown to predict success after ECV during short-term followup with a limited number of patients. ${ }^{9}$ Only 1 study examined the predictive value of the FWA after ECV. ${ }^{9}$ In this study patients with a higher FWA were more likely to maintain SR. Inclusion of these parameters in our model confirms these previous observations. ${ }^{18}$ However, the data represented here demonstrate that the DF is a stronger predictor of favourable outcome than SampEn and FWA.

\section{Clinical characteristics and ECG parameters}

The best clinical predictor for maintenance of SR was the use of AADs. Patients receiving AAD treatment had a significantly lower DF and SampEn than did patients not receiving AAD treatment. Although AADs affected some of the ECG-derived parameters, excluding patients on AADs did not significantly 
affect the predictive performance of the ECG parameters. This observation demonstrates that the predictive value of the ECG parameters is not based on the fact that AAD use prolongs the AF cycle and lowers the DF. Others showed that the DF was lowered not only by AAD treatment but also by advancing age. $^{11}$ We also observed this relation, especially after excluding patients receiving $A A D$ treatment $(R=-0.256, P<0.001)$. Interestingly, the FWA also becomes lower with advancing age $(R=-0.145, P=0.001)$. Possibly, progressive atrial fibrosis with increasing age or comorbidities accounts for the changes in these parameters. ${ }^{24}$ Studies using both, noninvasive and invasive measurements for AF cycle length have demonstrated that an increase in atrial fibrosis is associated with a decrease in DF. ${ }^{25,26}$ Furthermore, the amplitude of ECG parameters on the body surface could decrease as a result of an increasing amount of fibrosis, resulting in an enhanced degree of conduction block and electrical dissociation during AF. The exact pathophysiological meaning of the AF frequency and complexity parameters in patients with $A F$ certainly warrants further investigation.

\section{Future directions}

The parameters identified in this study are easy to implement in everyday clinical practice. These complexity and frequency parameters may be useful to choose the appropriate treatment in an individual patient. In patients with a low complexity and/or low DF, SR maintenance may be achieved even without treatment with AADs. In contrast, in patients with a high DF and /or high AF complexity, a more aggressive strategy for rhythm control may be required. We think early and appropriate treatment might improve rhythm outcome and might prevent cardiovascular complications. ${ }^{27}$ To prove this hypothesis additional large prospective clinical trials are needed.

\section{Limitations}

A limited number of patients with persistent AF experience self-terminating paroxysms of AF after ECV for persistent AF. There was no daily rhythm followup during the study period, and therefore we might have missed asymptomatic self-terminating episodes of AF. Furthermore, rhythm follow-up was not standardised during the entire follow-up. However, patients without a recurrence received a median of 4 rhythm follow-up visits during the 1-year study period without AF documented. The ECGs before ECV were recorded as part of routine clinical practice. Longer ECG recordings and recordings at alternative sites on the body surface or with a higher sampling frequencies might improve the predictive performance of these parameters. 
Predicting electrical cardioversion

\section{Conclusions}

ECG-derived atrial frequency and complexity parameters predict maintenance of SR after ECV during 1-year follow-up at least as good as clinical predictors. The DF is the best performing ECG parameter. Although the DF was affected by AAD treatment, excluding patients on AADs did not significantly lower the predictive performance captured by the ECG. A model containing ECG-derived parameters was able to predict SR maintenance 1 year after ECV, but prediction of early AF recurrences was less accurate. 


\section{Chapter 5}

\section{References}

1. Camm AJ, Kirchhof P, Lip GY, et al. Guidelines for the management of atrial fibrillation: the Task Force for the Management of Atrial Fibrillation of the European Society of Cardiology (ESC). European Heart Journal 2010;31:23692429.

2. Van Gelder IC, Hagens VE, Bosker HA, Kingma JH, Kamp O, Kingma T, Said SA, Darmanata JI, Timmermans AJ, Tijssen JG, Crijns HJ. A comparison of rate control and rhythm control in patients with recurrent persistent atrial fibrillation. The New England Journal of Medicine 2002;347:1834-1840.

3. Van Gelder IC, Crijns HJ, Tieleman RG, Brugemann J, De Kam PJ, Gosselink AT, Verheugt FW, Lie KI. Chronic atrial fibrillation. Success of serial cardioversion therapy and safety of oral anticoagulation. Archives of Internal Medicine 1996;156:2585-2592.

4. Crijns HJ, Weijs B, Fairley AM, et al. Contemporary real life cardioversion of atrial fibrillation: Results from the multinational RHYTHM-AF study. International Journal of Cardiology 2014;172:588-594.

5. Lafuente-Lafuente C, Valembois L, Bergmann JF, Belmin J. Antiarrhythmics for maintaining sinus rhythm after cardioversion of atrial fibrillation. The Cochrane Database of Systematic Reviews 2015;3:CD005049.

6. Van Gelder IC, Crijns HJ, Van Gilst WH, Verwer R, Lie KI. Prediction of uneventful cardioversion and maintenance of sinus rhythm from directcurrent electrical cardioversion of chronic atrial fibrillation and flutter. The American Journal of Cardiology 1991;68:41-46.

7. De Vos CB, Limantoro I, Pisters R, Delhaas T, Schotten U, Cheriex EC, Tieleman RG, Crijns HJ. The mechanical fibrillation pattern of the atrial myocardium is associated with acute and long-term success of electrical cardioversion in patients with persistent atrial fibrillation. Heart Rhythm 2014;11:1514-1521.

8. Lankveld TA, Zeemering S, Crijns HJ, Schotten U. The ECG as a tool to determine atrial fibrillation complexity. Heart 2014;100:1077-1084.

9. Alcaraz R, Hornero F, Rieta JJ. Noninvasive time and frequency predictors of long-standing atrial fibrillation early recurrence after electrical cardioversion. Pacing and Clinical Electrophysiology 2011;34:1241-1250.

10. Holmqvist F, Stridh M, Waktare JE, Sornmo L, Olsson SB, Meurling CJ. Atrial fibrillatory rate and sinus rhythm maintenance in patients undergoing cardioversion of persistent atrial fibrillation. European Heart Journal 2006;27:2201-2207.

11. Bollmann A, Tveit A, Husser D, Stridh M, Sornmo L, Smith P, Olsson SB. Fibrillatory rate response to candesartan in persistent atrial fibrillation. Europace 2008;10:1138-1144.

12. Schotten $U$, Maesen B, Zeemering S. The need for standardization of timeand frequency-domain analysis of body surface electrocardiograms for assessment of the atrial fibrillation substrate. Europace 2012;14:1072-1075. 
13. Bonizzi P, Zeemering S, Karel JM, Di Marco LY, Uldry L, Van Zaen J, Vesin JM, Schotten U. Systematic comparison of non-invasive measures for the assessment of atrial fibrillation complexity: a step forward towards standardization of atrial fibrillation electrogram analysis. Europace 2015;17:318-325.

14. Alcaraz R, Rieta JJ. Adaptive singular value cancelation of ventricular activity in single-lead atrial fibrillation electrocardiograms. Physiological Measurement 2008;29:1351-1369.

15. Bollmann A, Husser D, Steinert R, Stridh M, Soernmo L, Olsson SB, Polywka D, Molling J, Geller C, Klein HU. Echocardiographic and Electrocardiographic Predictors for Atrial Fibrillation Recurrence Following Cardioversion. Journal of Cardiovascular Electrophysiology 2003;14:S162-S165.

16. Meurling CJ, Roijer A, Waktare JE, Holmqvist F, Lindholm CJ, Ingemansson MP, Carlson J, Stridh M, Sornmo L, Olsson SB. Prediction of sinus rhythm maintenance following DC-cardioversion of persistent atrial fibrillation - the role of atrial cycle length. BMC Cardiovascular Disorders 2006;6:11.

17. Efremidis $M$, Alexanian IP, Oikonomou D, Manolatos D, Letsas KP, Pappas LK, Gavrielatos G, Vadiaka M, Mihas CC, Filippatos GS, Sideris A, Kardaras F. Predictors of atrial fibrillation recurrence in patients with long-lasting atrial fibrillation. The Canadian Journal of Cardiology 2009;25:e119-124.

18. Alcaraz R, Rieta JJ. Time and frequency recurrence analysis of persistent atrial fibrillation after electrical cardioversion. Physiological Measurement 2009;30:479-489.

19. Van Gelder IC, Tuinenburg AE, Schoonderwoerd BS, Tieleman RG, Crijns HJ. Pharmacologic versus direct-current electrical cardioversion of atrial flutter and fibrillation. The American Journal of Cardiology 1999;84:147R-151R.

20. Tieleman RG, Van Gelder IC, Crijns HJ, De Kam PJ, Van Den Berg MP, Haaksma J, Van Der Woude HJ, Allessie MA. Early recurrences of atrial fibrillation after electrical cardioversion: a result of fibrillation-induced electrical remodeling of the atria? Journal of the American College of Cardiology 1998;31:167-173.

21. Kirchhof $P$, Andresen $D$, Bosch $R$, Borggrefe $M$, Meinertz $T$, Parade $U$, Ravens U, Samol A, Steinbeck G, Treszl A, Wegscheider K, Breithardt G. Short-term versus long-term antiarrhythmic drug treatment after cardioversion of atrial fibrillation (Flec-SL): a prospective, randomised, open-label, blinded endpoint assessment trial. Lancet 2012;380:238-246.

22. Ahmed S, Rienstra M, Crijns HJ, Links TP, Wiesfeld AC, Hillege HL, Bosker HA, Lok DJ, Van Veldhuisen DJ, Van Gelder IC. Continuous vs episodic prophylactic treatment with amiodarone for the prevention of atrial fibrillation: a randomized trial. JAMA 2008;300:1784-1792.

23. Abacherli R, Leber R, Lemay $M$, Vesin JM, van Oosterom A, Schmid HJ, Kappenberger L. Development of a toolbox for electrocardiogram-based interpretation of atrial fibrillation. Journal of Electrocardiology 2009;42:517521. 


\section{Chapter 5}

24. Schotten U, Verheule S, Kirchhof P, Goette A. Pathophysiological mechanisms of atrial fibrillation: a translational appraisal. Physiological Reviews 2011;91:265-325.

25. Walters TE, Teh AW, Spence S, Kistler PM, Morton JB, Kalman JM. Relationship between the electrocardiographic atrial fibrillation cycle length and left atrial remodeling: a detailed electroanatomic mapping study. Heart Rhythm 2014;11:670-676.

26. Koduri H, Ng J, Cokic I, Aistrup GL, Gordon D, Wasserstrom JA, Kadish AH, Lee R, Passman R, Knight BP, Goldberger JJ, Arora R. Contribution of fibrosis and the autonomic nervous system to atrial fibrillation electrograms in heart failure. Circulation Arrhythmia and Electrophysiology 2012;5:640-649.

27. Nattel S, Guasch E, Savelieva I, et al. Early management of atrial fibrillation to prevent cardiovascular complications. European Heart Journal 2014;35:14481456. 


\section{Supplements}

\begin{tabular}{|c|c|c|c|c|}
\hline & $\begin{array}{c}\begin{array}{c}\text { ECV failure } \\
\mathrm{N}=50\end{array}\end{array}$ & $\begin{array}{c}\text { Early AF } \\
\text { recurrence } \\
\mathrm{N}=196\end{array}$ & $\begin{array}{c}\text { Late } \mathrm{AF} \\
\text { recurrence } \\
\mathrm{N}=95\end{array}$ & $\begin{array}{c}\mathrm{SR} \\
\text { maintenance } \\
\mathrm{N}=161\end{array}$ \\
\hline Age (years) & $63 \pm 10$ & $67 \pm 8$ & $65 \pm 11$ & $67 \pm 12$ \\
\hline Male & $29(58 \%)$ & 135 (69\%) & $74(78 \%)$ & $116(72 \%)$ \\
\hline BMI & $30.3 \pm 6.5$ & $28.8 \pm 5.0$ & $28.8 \pm 4.8$ & $28.6 \pm 5.0$ \\
\hline Hypertension & $30(60 \%)$ & $118(60 \%)$ & $55(58 \%)$ & $99(62 \%)$ \\
\hline Diabetes & $10(20 \%)$ & 25 (13\%) & $6(6 \%)$ & $16(10 \%)$ \\
\hline CAD & $9(18 \%)$ & $56(29 \%)$ & $26(27 \%)$ & $43(27 \%)$ \\
\hline PM/ICD & $1(2 \%)$ & $10(5 \%)$ & $10(11 \%)$ & $8(5 \%)$ \\
\hline Heart Failure & $17(34 \%)$ & $50(26 \%)$ & $21(22 \%)$ & $43(33 \%)$ \\
\hline Stroke & $5(10 \%)$ & $23(12 \%)$ & $11(12 \%)$ & $10(6 \%)$ \\
\hline COPD & $6(12 \%)$ & $24(12 \%)$ & $9(10 \%)$ & $10(6 \%)$ \\
\hline OSAS & $5(10 \%)$ & $9(5 \%)$ & $9(10 \%)$ & $8(5 \%)$ \\
\hline $\mathrm{CHA}_{2} \mathrm{DS}_{2}$-VASc & $2.7 \pm 1.9$ & $2.6 \pm 1.6$ & $2.3 \pm 1.6$ & $2.5 \pm 1.4$ \\
\hline Total AF duration & $7[3-41]$ & $8[3-49]$ & $7[3-44]$ & $7[3-44]$ \\
\hline AF-episode (Months) & $3[2-7]$ & $3[2-6]$ & $3[2-5]$ & $3[1-5]$ \\
\hline Previous ECV & $15(30 \%)$ & $55(28 \%)$ & $29(31 \%)$ & $39(24 \%)$ \\
\hline Diuretic & $25(50 \%)$ & $94(48 \%)$ & $34(36 \%)$ & $80(50 \%)$ \\
\hline ACE or ARB & 35 (70\%) & $134(68 \%)$ & 67 (71\%) & 112 (70\%) \\
\hline Beta-blocker & 36 (72\%) & 151 (77\%) & 74 (78\%) & 132 (82\%) \\
\hline Digoxin & $15(30 \%)$ & 61 (31\%) & $19(20 \%)$ & $44(27 \%)$ \\
\hline Statin & $23(46 \%)$ & 96 (49\%) & 42 (44\%) & 88 (55\%) \\
\hline Any AAD & $10(20 \%)$ & 38 (19\%) & $25(26 \%)$ & $59(37 \%)$ \\
\hline Amiodarone & $6(12 \%)$ & $25(13 \%)$ & $10(11 \%)$ & $39(24 \%)$ \\
\hline Flecainide & $0(0 \%)$ & $3(2 \%)$ & $3(3 \%)$ & $5(3 \%)$ \\
\hline Sotalol & $4(8 \%)$ & $11(6 \%)$ & $11(12 \%)$ & $16(10 \%)$ \\
\hline LVEF (\%) & $49 \pm 14$ & $50 \pm 12$ & $49 \pm 12$ & $50 \pm 13$ \\
\hline LAV (mL) & $91 \pm 28$ & $94 \pm 30$ & $104 \pm 30$ & $95 \pm 30$ \\
\hline $\operatorname{RAV}(\mathrm{mL})$ & $81 \pm 32$ & $83 \pm 32$ & $86 \pm 29$ & $76 \pm 27$ \\
\hline
\end{tabular}

Supplement table A: Baseline characteristics per recurrence group. Anti-arrhythmic drug (AAD), angiotensin receptor blocker (ARB) Atrial fibrillation (AF), body mass index (BMI), coronary artery disease (CAD), chronic obstructive pulmonary disease (COPD), electrical cardioversion $(E C V)$, implantable cardiac defibrillator (ICD), left atrial volume (LAV), left ventricular ejection fraction (LVEF), pacemaker (PM), right atrial volume (RAV), sinus rhythm (SR). 


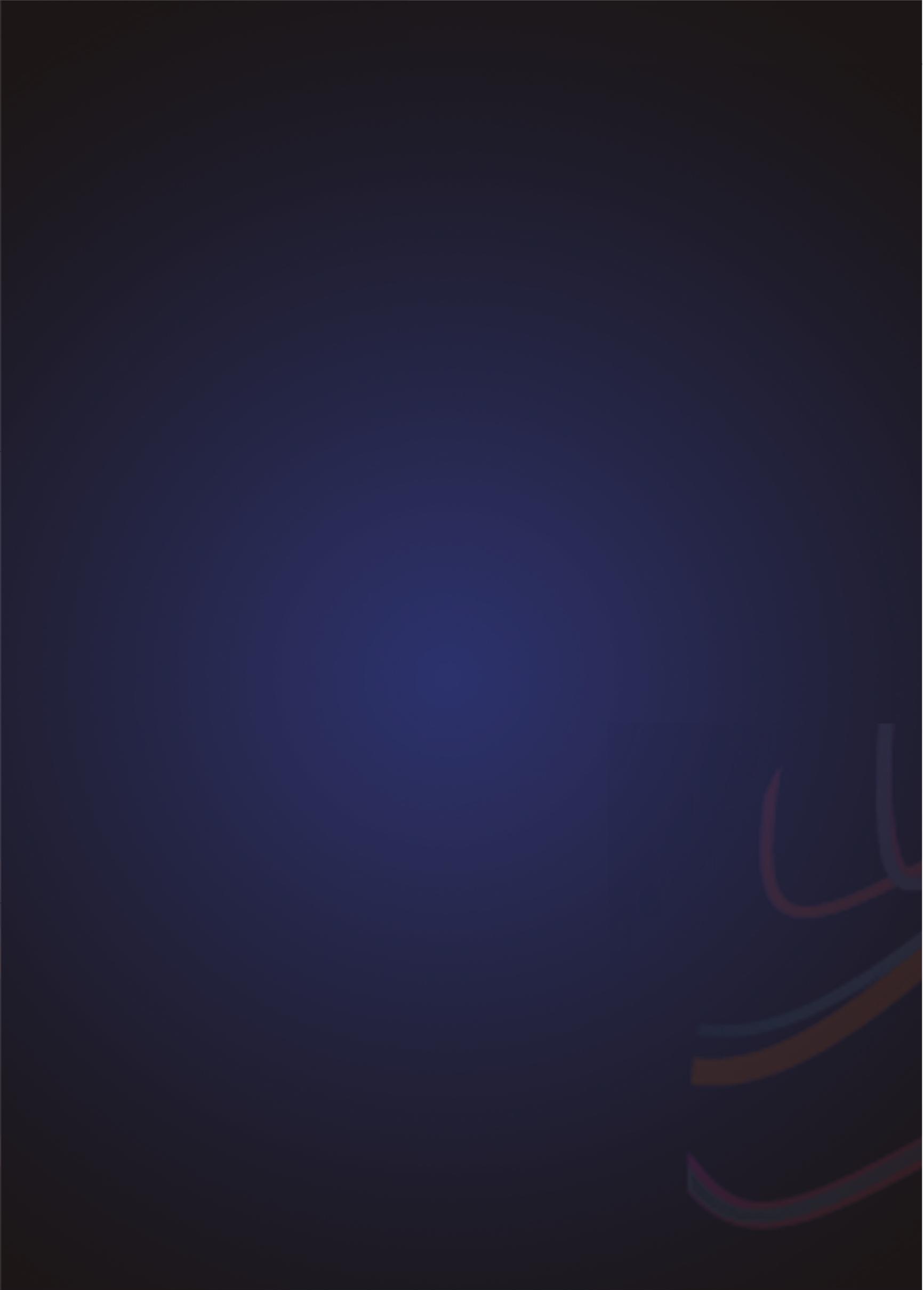




\section{Editorial}

Intervention for persistent atrial fibrillation: Prediction of treatment response

Damian P Redfearn, Javad Hashemi 
Atrial fibrillation (AF) is an epidemic with spiraling costs and health care burden ${ }^{1}$, yet it is complex disease that consistently frustrates attempts to better understand the basic pathophysiological mechanism of perpetuation. The complex interplay between triggers and electrophysiological substrate is dynamic and subject to change over time. ${ }^{2}$ The surface ECG has long been used to inform atrial substrate in patients with a diagnosis, or at risk of developing AF. Clinicians have used the $P$ wave to inform risk since the introduction of the modern 12 lead ECG. ${ }^{3}$ However the role of $\mathrm{P}$ wave analysis is limited to sinus rhythm and has no role during assessment of sustained AF where the goal is to inform candidacy for intervention. There have been a wide variety of signal processing techniques employed for surface ECG analysis, these can be broadly grouped into body surface mapping ${ }^{4,5}$ and standard 12 lead recordings. ${ }^{6}$ Digitized recording and subtraction of the surface QRS allows for inspection of uninterrupted atrial activity during AF and is a relatively simple and efficient technique. ${ }^{7}$ The resultant signal can then be filtered and analyzed in the time and frequency domains. The derived variables have been linked with atrial refractory period $^{8}$ and catheter ablation or cardioversion outcomes. ${ }^{9,10}$

\section{Time and Frequency Domain Analysis}

Time and frequency domain analysis of ECG signals allows for an objective and reproducible metric that can be assessed, quantified and analysed. During AF, atrial activity is described as chaotic and random, with no apparent pattern. This makes the time-domain analysis of the AF electrograms challenging since time-domain approaches work best when the signals have defined repeating patterns (as in atrial tachycardia or flutter). Thus, researchers have shifted to frequency domain analyses to avoid dealing with the complexity in the chaotic nature of electrogram recordings in time-domain during AF. Contemporary mapping and AF research suggest higher frequency regions are associated with perpetuation of AF and increased risk of recurrence. ${ }^{11,12}$ Fourier transform is a mathematical operation that converts a time-domain signal to a frequency spectrum. This is done by breaking the signal into a finite number of sinusoidal waveforms with different amplitude and frequency. The summation of all these sinusoidal waveforms will approximate the original electrogram in the time domain. The amplitude of each of these sinusoidal waveforms represents the contribution of a specific frequency in constructing the original electrogram. Thus, the waveform with the highest amplitude is expected to have the highest contribution in constructing the signal and the best candidate if we were to approximate the electrogram with only one sinusoidal waveform. Dominant frequency (DF) is the frequency of the sinusoidal waveform with the highest amplitude and is an effective estimator of the activation rate; the more organised the signal the more robust the estimate. ${ }^{13}$ The surface ECG 
frequency spectrum then is a summation and representation of multiple sources that perpetuate AF. The DF does not necessarily represent the fastest source but the frequency (or fibrillatory rate) that contributes most to the signal.

Summary of the study by Lankveld et al

In this issue of the journal Schotten and colleagues describe a prospective cohort of 502 patients undergoing electrical cardioversion (ECV) for persistent AF. ${ }^{14}$ Twelve lead digital ECG recordings were acquired prior to cardioversion and patients followed for one year. Offline analysis was performed on each of the 12 leads to determine optimal parameters associated with maintenance of sinus rhythm (SR). The group employed a variety of contemporary signal processing techniques in both time and frequency domains and observed an association with sustained SR and lower DF, particularly from lead V6.

What does this study add?

Recurrence of AF after ECV is a complex process, it includes the variable of triggers and the ability of the atrium to dynamically remodel and become less vulnerable to AF recurrence with time. ${ }^{2}$ Nonetheless it lacks the confounders from the technical aspects of an ablation procedure and thus represents a clearer picture of what estimation of atrial substrate via surface ECG at a single time point might deliver. This study and technique is remarkable for its simplicity, ease of use, demonstrated reproducibility and sample size. As a 'snapshot' of atrial substrate it affords the potential for a simple ECG derived metric associated with favourable outcomes. Currently, prediction of response relies on demographic features or sophisticated and expensive imaging of the atria. ${ }^{15,16}$ Magnetic resonance imaging is promising but requires significant expertise in image processing and has limited availability. With the increased interest and focus on catheter ablation of persistent AF, a simple ECG measure, validated in the ECV cohort would be a welcome tool. Recent work has cast doubt on the role of substrate modification in addition to pulmonary vein isolation, STAR-AF II

suggested that additional substrate ablation appeared to be non contributary $i^{17}$ however other studies have found substrate ablation alone or in combination with isolation to be helpful. ${ }^{18,19}$ It remains unclear what surface DF represents or how it might inform ablation strategy, but this is the next logical step. Frequency domain parameters have been limited by poor reproducibility and conflicting results; is it really conceivable that a low resolution, 10 second recording could provide

detailed insight into atrial substrate? The reproducibility of the metric argues this is a real measure and as such warrants further investigation as a potential 


\section{Editorial}

clinical tool. There is a clear need for better risk stratification and prediction measures than current demographics. These data from an ECV cohort support the notion that such simple measures might exist and further should be explored in contemporary ablation settings. 


\section{References}

1. Patel NJ, Deshmukh A, Pant S, et al. Contemporary trends of hospitalization for atrial fibrillation in the United States, 2000 through 2010: implications for healthcare planning. Circulation 2014;129:2371-2379.

2. Allessie M, Ausma J, Schotten U. Electrical, contractile and structural remodeling during atrial fibrillation. Cardiovascular Research 2002;54:230246.

3. Brody DA, Woolsey MD, Arzbaecher RC. Application of computer techniques to the detection and analysis of spontaneous $\mathrm{P}$-wave variations. Circulation 1967;36:359-371.

4. Haissaguerre $M$, Hocini $M$, Shah AJ, Derval $N$, Sacher $F$, Jais $P$, Dubois R. Noninvasive panoramic mapping of human atrial fibrillation mechanisms: a feasibility report. Journal of Cardiovascular Electrophysiology 2013;24:711717.

5. Guillem MS, Climent AM, Millet J, Arenal A, Fernandez-Aviles F, Jalife J, Atienza F, Berenfeld O. Noninvasive localization of maximal frequency sites of atrial fibrillation by body surface potential mapping. Circulation Arrhythmia and Electrophysiology 2013;6:294-301.

6. Matsuo S, Lellouche N, Wright M, et al. Clinical predictors of termination and clinical outcome of catheter ablation for persistent atrial fibrillation. Journal of the American College of Cardiology 2009;54:788-795.

7. Alcaraz R, Rieta JJ. Adaptive singular value cancelation of ventricular activity in single-lead atrial fibrillation electrocardiograms. Physiological Measurement 2008;29:1351-1369.

8. Bollmann A, Kanuru NK, McTeague KK, Walter PF, DeLurgio DB, Langberg JJ. Frequency analysis of human atrial fibrillation using the surface electrocardiogram and its response to ibutilide. The American Journal of Cardiology 1998;81:1439-1445.

9. Di Marco LY, Raine D, Bourke JP, Langley P. Characteristics of atrial fibrillation cycle length predict restoration of sinus rhythm by catheter ablation. Heart Rhythm 2013;10:1303-1310.

10. Holmqvist F, Stridh M, Waktare JE, Sornmo L, Olsson SB, Meurling CJ. Atrial fibrillatory rate and sinus rhythm maintenance in patients undergoing cardioversion of persistent atrial fibrillation. European Heart Journal 2006;27:2201-2207.

11. Berenfeld O, Ennis S, Hwang E, Hooven B, Grzeda K, Mironov S, Yamazaki M, Kalifa J, Jalife J. Time- and frequency-domain analyses of atrial fibrillation activation rate: the optical mapping reference. Heart Rhythm 2011;8:17581765.

12. Atienza F, Almendral J, Jalife J, Zlochiver S, Ploutz-Snyder R, Torrecilla EG, Arenal A, Kalifa J, Fernandez-Aviles F, Berenfeld O. Real-time dominant frequency mapping and ablation of dominant frequency sites in atrial fibrillation with left-to-right frequency gradients predicts long-term maintenance of sinus rhythm. Heart Rhythm 2009;6:33-40. 


\section{Editorial}

13. $\mathrm{Ng} \mathrm{J,} \mathrm{Kadish} \mathrm{AH,} \mathrm{Goldberger} \mathrm{JJ.} \mathrm{Effect} \mathrm{of} \mathrm{electrogram} \mathrm{characteristics} \mathrm{on} \mathrm{the}$ relationship of dominant frequency to atrial activation rate in atrial fibrillation. Heart Rhythm 2006;3:1295-1305.

14. Lankveld T, de Vos CB, Limantoro I, Zeemering S, Dudink E, Crijns HJ, Schotten U. Systematic Analysis of ECG Predictors for Sinus Rhythm Maintenance Following Electrical Cardioversion for Persistent Atrial Fibrillation. Heart Rhythm 2016.

15. Jacobs V, May HT, Bair TL, Crandall BG, Cutler M, Day JD, Weiss JP, Osborn JS, Muhlestein JB, Anderson JL, Mallender C, Bunch TJ. The impact of risk score (CHADS2 versus CHA2DS2-VASc) on long-term outcomes after atrial fibrillation ablation. Heart Rhythm 2015;12:681-686.

16. Han FT, Akoum N, Marrouche N. Value of magnetic resonance imaging in guiding atrial fibrillation management. The Canadian Journal of Cardiology 2013;29:1194-1202.

17. Verma A, Jiang $\mathrm{CY}$, Betts $\mathrm{TR}$, et al. Approaches to catheter ablation for persistent atrial fibrillation. The New England Journal of Medicine 2015;372:1812-1822.

18. Atienza F, Almendral J, Ormaetxe JM, et al. Comparison of radiofrequency catheter ablation of drivers and circumferential pulmonary vein isolation in atrial fibrillation: a noninferiority randomized multicenter RADAR-AF trial. Journal of the American College of Cardiology 2014;64:2455-2467.

19. Seitz J, Horvilleur J, Curel L, et al. Active or passive pulmonary vein in atrial fibrillation: is pulmonary vein isolation always essential? Heart rhythm 2014;11:579-586. 



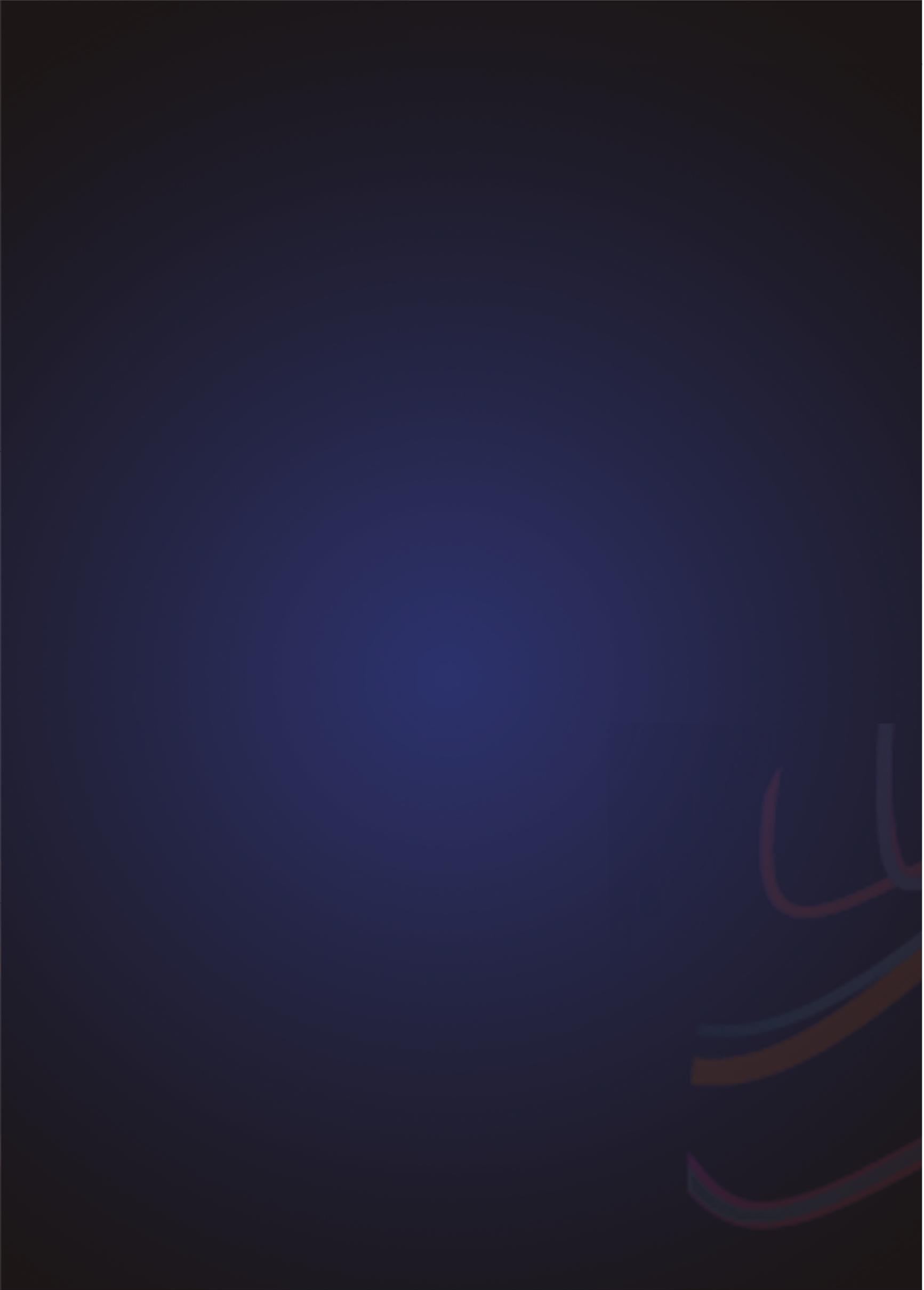




\section{Chapter 6}

Atrial fibrillation complexity parameters derived from surface ECG predict procedural outcome and long-term follow-up of stepwise catheter ablation for atrial fibrillation

Theo Lankveld, Stef Zeemering, Daniel Scherr, Pawel Kuklik, Boris A Hoffmann, Stephan Willems, Burkert Pieske, Michel Haïssaguerre, Pierre Jaïs, Harry J Crijns and Ulrich Schotten Circulation: Arrhythmia and Electrophysiology 2016;9:e003354 
Chapter 6

\begin{abstract}
Background: The success rate of catheter ablation for persistent atrial fibrillation (AF) is still far from satisfactory. Identification of patients who will benefit from ablation is highly desirable. We investigated the predictive value of noninvasive AF complexity parameters derived from standard 12-lead ECGs for AF termination and long-term success of catheter ablation and compared them with clinical predictors.
\end{abstract}

Methods and results: The study included a training (93 patients) and validation set (81 patients) of patients with persistent AF undergoing stepwise radiofrequency ablation. In the training set AF terminated in $81 \%$ during CA, $77 \%$ were in sinus rhythm after 6 years and multiple ablations. ECG-derived complexity parameters were determined from a baseline $10 \mathrm{~s}$ 12-lead ECG. Prediction of AF termination was similar using only ECG (cross-validated mean area under the curve $(A \cup C), 0.76 \pm 0.15$ ) or only clinical parameters (mean AUC, $0.75 \pm 0.16)$. The combination improved prediction to a mean AUC of $0.79 \pm 0.13$. Using a combined model of ECG and clinical parameters, SR at long-term follow-up could be predicted with a mean AUC $0.71 \pm 0.12$. In the validation set AF terminated in 57\%, 61\% were in SR after 4.6 years. The combined models predicted termination with an AUC of 0.70 and SR at longterm follow-up with an AUC of 0.61. Overall, fibrillation-wave amplitude provided the best rhythm prediction.

Conclusions: The predictive performance of ECG-derived AF complexity parameters for AF termination and long-term success of catheter ablation in patients with persistent AF is at least as good as known clinical predictive parameters, with fibrillation-wave amplitude as the best predictor. 


\section{Introduction}

Catheter ablation (CA) is a successful and widely used therapy in patients with paroxysmal atrial fibrillation (AF). ${ }^{1}$ Recently, a systematic meta-analysis confirmed that also patients with persistent AF benefit more from CA than from anti-arrhythmic drug (AAD) therapy in terms of maintenance of sinus rhythm (SR). ${ }^{2}$ Depending on ablation procedure and rhythm follow-up, reported long-term success rates range from only $25 \%$ up to $80 \%$. $^{3-6}$ Thus, development of diagnostic means to identify patients likely responding to CA are highly desirable. Several parameters have been suggested that predict success of $C A$ in patients with persistent $A F$, for example the duration of the AF episode or left atrial dimensions. ${ }^{7,8}$ However, the exact duration of the AF episode is often difficult to determine and reports on predictive values of echocardiographic parameters are conflicting. A standard 12-lead ECG is available in every patient with persistent AF before ablation and may reflect the complexity of fibrillatory conduction in the atria during AF. ${ }^{9}$

Not only AF but also structural heart diseases cause a steady and progressive process of structural remodelling characterized by atrial fibrosis with electrical uncoupling between muscle bundles. ${ }^{10}$ The resulting increase in atrial conduction disturbances and thus complexity of the AF conduction pattern likely underlies the loss of efficacy of antiarrhythmic drug treatment and recurrence after $\mathrm{CA}^{10}{ }^{10}$ Therefore, we sought to investigate AF complexity parameters derived from a standard 12-lead ECG to predict ablation outcome and compare them with known clinical predictors. Many different AF complexity parameters have been studied to predict rhythm outcome in different patient populations. However, in most of these studies, follow-up was limited or only 1 AF complexity parameter or lead was studied. ${ }^{9}$ Because of this lack of standardization of advanced ECG analysis, the aim of this study was to compare different time and frequency parameters and determine which parameters and combination of leads provide the optimal outcome prediction. In a training set of patients with persistent AF undergoing CA, we determined the best ECG parameters for prediction of acute outcome (i.e. termination during the index ablation) and SR after long-term follow-up. Secondly, we validated these parameters in an external ablation cohort of similar persistent AF patients. Finally, we compared the identified AF complexity parameters with known clinical predictors of success for both acute and long-term outcome after CA for AF. 
Chapter 6

\section{Methods}

\section{Patient populations}

\section{Training cohort}

We included 93 patients undergoing de novo stepwise radiofrequency CA for persistent AF between July 2004 and October 2007 at the Hôpital Cardiologique du Haut Lévêque, Bordeaux, France. Patients needed to be aged between 18 and 80 years and with AF for at least 1 month at the start of the procedure. The patients are a sample of consecutive patients in whom a digitally recorded 12-lead ECG on the day of ablation was available. The stepwise ablation protocol used was described in detail before. ${ }^{11}$ After the procedure, a blanking period of 1 month was applied and repeat ablations were offered if a patient had a symptomatic or asymptomatic recurrence of a supraventricular arrhythmia. Patients received follow-up with 24-hour Holter monitoring at 1, 3, 6, and 12 months after each ablation. Between October 2011 and May 2012, all patients received additional 7 days of Holter monitoring to complete the follow-up. All patients gave written informed consent, and the study was approved by the institutional review board of the University Hospital of Bordeaux.

\section{Validation cohort}

We validated the prediction models in an external cohort. The validation cohort consisted of 81 consecutive patients with persistent AF undergoing de novo sequential ablation between December 2006 and May 2009 at the University Hospital Eppendorf, Hamburg, Germany and presented for a second procedure for AF or AT recurrence. The ablation protocol used has been described in detail previously. ${ }^{12}$ A digitally stored ECG at the day of the first procedure had to be available. Patients with SR, atrial flutter, or atrial tachycardia at the start of the procedure and patients with an ECG not suitable for analysis were excluded. Holter monitoring was performed every 6 months. This study was approved by the institutional review board. All patients provided written informed consent. In both the training and the validation cohort, the ECG data were collected and analyzed retrospectively. 


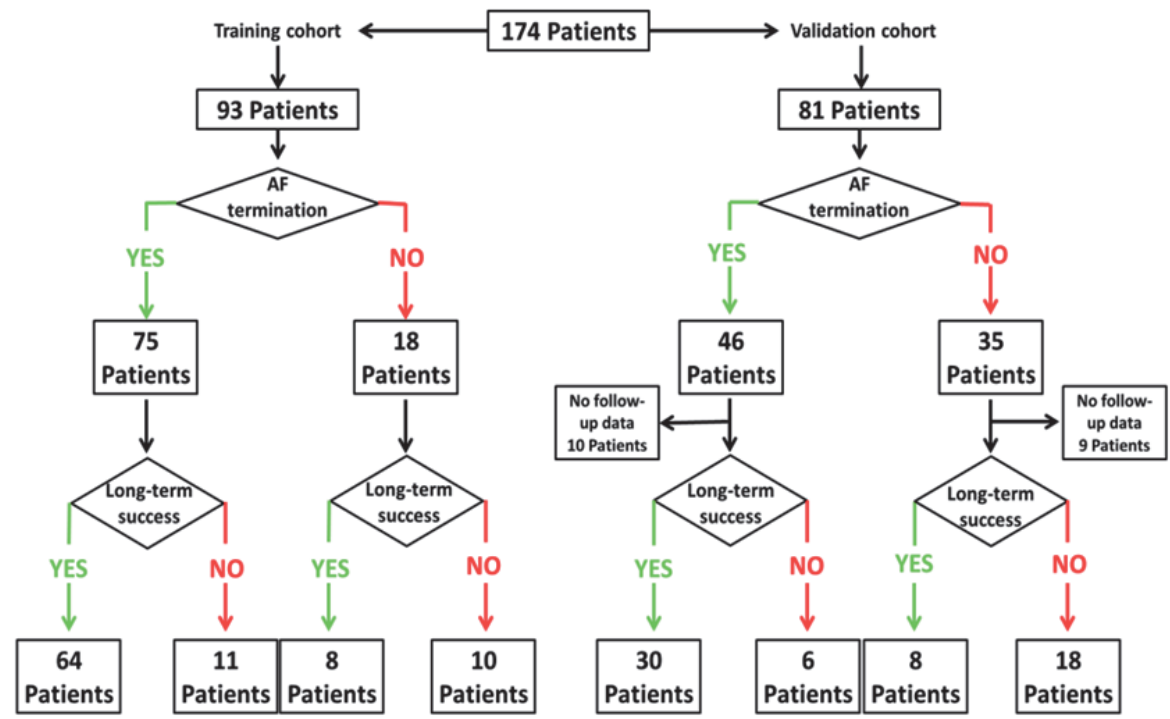

Figure 1: This figure shows the study overview. The left part of the figure shows the training cohort and the right part the validation cohort. In the validation cohort there was no follow-up data available in 19 patients.

\section{End points}

In both cohorts, the primary procedural endpoint was termination of AF without AAD or direct current cardioversion. Termination of AF was defined as conversion of AF into atrial tachycardia, atrial flutter or SR. In both cohorts, the primary long-term end point was defined as freedom from any documented symptomatic or asymptomatic atrial arrhythmia (AF, atrial flutter or atrial tachycardia) lasting > $30 \mathrm{~s}$ after the last ablation procedure with or without AADs.

\section{ECG analysis}

Surface ECGs were digitally recorded with a $1000-\mathrm{Hz}$ sampling frequency during the entire procedure using an EP (electrophysiology) recording system (LabSystem PRO; Bard EP, Lowell, MA). A 60-s 12-lead ECG recording the start of the procedure was exported for analysis, from which a 10-s ECG recording was selected for primary analysis. ECGs were filtered using a digital zero-phase band-pass filter between 1 and $100 \mathrm{~Hz}$ (third order Chebyshev, 20$\mathrm{dB}$ stop-band attenuation) to remove baseline wandering and suppress highfrequency noise. Powerline interference, if present, was removed using a 
second order infinite impulse response notch filter, centered around $50 \mathrm{~Hz}$. Ventricular signals were cancelled using a modified version of the adaptive singular value QRST cancellation. This method groups QRST windows before QRST template computation, using hierarchical clustering based on window correlation. $^{13}$ During QRST cancellation, the signals were temporarily upsampled to $2000 \mathrm{~Hz}$ to improve alignment of the QRST templates. Before the analysis an additional $3 \mathrm{~Hz}$ high-pass filter was used to avoid interference caused by the (possible residual) T-wave.

The parameters computed on the ECG covered both the frequency domain and time domain. Frequency domain parameters included dominant frequency (DF), ${ }^{14}$ organization index (OI) ${ }^{15}$ and spectral entropy (SE). ${ }^{15}$ Time domain parameters consisted of the mean fibrillation wave amplitude (FWA ${ }^{16}$ and sample entropy (SampEn). ${ }^{17}$ For the frequency domain parameters, a frequency power spectrum was estimated using Welch power spectral density estimate (3 segments; 4096 points; 50\% overlap). The DF was defined as the frequency with the highest power between 3 and $12 \mathrm{~Hz}$ in this frequency spectrum. For the OI, the areas between the 2 largest peaks of the frequency spectrum were calculated using a $1-\mathrm{Hz}$ frequency interval centered on each peak. The Ol was defined as the ratio of the cumulative areas of these 2 largest peaks and the area of the entire power spectrum. To compute the SE, the spectrum was first normalized to produce a probability mass function, on which Shannon entropy was calculated to give an estimate of SE. For FWA, the mean amplitude of the extracted atrial signal was used. F waves were detected using peak detection with a minimal distance between 2 peaks of $100 \mathrm{~ms}$ and a zero crossing within each $F$ wave. SampEn is a nonlinear regularity index. SampEn is calculated on the main atrial wave calculated by filtering with a $1-\mathrm{Hz}$ band around the DF. The number of samples used for SampEn computation was 3 within a tolerance of $r=0.35$ times the SE.

\section{Endocardial signals}

Endocardial signals were recorded from the left atrial appendage (LAA) and right atrial appendage (RAA) between ablation steps using a 10-pole circumferential mapping catheter (Lasso; Biosense-Webster, Diamond Bar, CA, USA) or a quadripolar, irrigated tip ablation catheter (ThermoCool; BiosenseWebster, Diamond Bar, CA, USA). The signals were exported from the EP recording system and imported in custom-made MatLab (R2013a, MathWorks Inc. Natick, MA, USA) software. To preprocess the signals for robust DF measurements, we used the method suggested by $\mathrm{Ng}$ and Goldberger. ${ }^{18}$ First, the endocardial signals were filtered using a $40-250 \mathrm{~Hz}$ band-pass filter. The 
remaining signal was then rectified followed by a $20 \mathrm{~Hz}$ low-pass filter step. ${ }^{18}$ On this filtered signal, a fast Fourier transform was performed in order to provide a power spectrum on which the DF could be calculated. These invasive recordings were compared with simultaneous ECG recordings.

\section{Statistical analysis}

Statistical analyses were performed using IBM SPSS statistics 21 and MatLab R2013a. Continuous variables are reported as mean \pm SD or median and range. Continuous variables were tested for normality using the Kolmogorov-Smirnov test. Comparison between groups was performed using a Student $t$ test or a Mann-Whitney $U$ test in the case of a bimodal end point; the latter test was for non-normally distributed data. Categorical variables are reported as number and percentage and are compared using the Fisher exact test. To build the prediction models, a forward stepwise logistic regression analysis with a 5fold cross validation was used. Cross validation was performed to get unbiased estimates of the model fit in the training set. Only parameters with a univariate $P$ value $\leq 0.10$ were included in the models. For validation of the models, we used the same parameters and coefficients as identified in the initial training set. Correlations between endocardial and surface DF are calculated using Pearson's correlation. Differences between ablation steps in patients with right-sided ablation are calculated using repeated measures ANOVA with Bonferroni correction for multiple comparisons. A $P$ value of $<0.05$ was considered statistically significant.

\section{Results}

The training set comprised 93 patients. In 75 patients (81\%) the procedural endpoint of AF termination was achieved, and 72 patients $(77 \%)$ were in SR at the end of the median follow-up period of 6 [5 - 6.7] years with a median of 2 [1 - 3] procedures. The validation cohort consisted of 81 patients, AF terminated in 46 patients (57\%). In 62 patients of the validation cohort longterm follow-up data was available. After a median follow-up of 4.6 [4.3 - 5] years and 2 [2 - 4] ablations 38 patients (61\%) were in SR. A study-overview is presented in figure 1 , and the baseline characteristics of both cohorts are provided in table 1. 


\begin{tabular}{|c|c|c|c|}
\hline & $\begin{array}{c}\text { Training } \\
\mathbf{N}=93\end{array}$ & $\begin{array}{c}\text { Validation } \\
\mathrm{N}=\mathbf{8 1}\end{array}$ & $P$ value \\
\hline Age (y) & $57 \pm 11$ & $60 \pm 9$ & 0.018 \\
\hline Male gender & 77 (83\%) & $66(81 \%)$ & 0.821 \\
\hline BMI & $26.7 \pm 3.8$ & $28.3 \pm 4.4$ & 0.033 \\
\hline Hypertension & $36(39 \%)$ & $59(73 \%)$ & $<0.001$ \\
\hline CAD & $13(14 \%)$ & $14(17 \%)$ & 0.548 \\
\hline HF & $21(23 \%)$ & $20(25 \%)$ & 0.743 \\
\hline Diabetes Mellitus & $8(9 \%)$ & $5(6 \%)$ & 0.543 \\
\hline Stroke & $6(6 \%)$ & $8(9 \%)$ & 0.407 \\
\hline Total AF duration (months) & $66[36-120]$ & $60[24-108]$ & 0.078 \\
\hline $\begin{array}{l}\text { Current AF episode } \\
\text { (months) }\end{array}$ & $13[7-24]$ & $10[4-24]$ & 0.059 \\
\hline Current use Amiodarone & $24(26 \%)$ & $30(37 \%)$ & 0.110 \\
\hline LVEF (\%) & $58 \pm 13$ & $57 \pm 12$ & 0.860 \\
\hline LA diameter (mm) & $48 \pm 8$ & $47 \pm 6$ & 0.617 \\
\hline Procedure time (min) & $260 \pm 72$ & $223 \pm 54$ & $<0.001$ \\
\hline RF time (min) & $95 \pm 30$ & $92 \pm 23$ & 0.415 \\
\hline Termination during ablation & $75(81 \%)$ & $46(57 \%)$ & 0.001 \\
\hline Long-term SR & $72(77 \%)$ & $38(61 \%)^{*}$ & 0.030 \\
\hline Follow-up duration (y) & $6[5-6.7]$ & $4.6[4.3-5]$ & $<0.001$ \\
\hline Number procedures & $2[1-3]$ & $2[2-4]$ & $<0.001$ \\
\hline
\end{tabular}

*Out of 62 patient, 19 patients were lost to follow-up.

Table 1: Baseline characteristics of the training and validation cohorts. Atrial fibrillation (AF), body mass index (BMI), coronary artery disease (CAD), heart failure (HF), left atrial (LA), left ventricular ejection fraction (LVEF), radiofrequency (RF), sinus rhythm (SR).

\section{Prediction of AF termination during ablation}

The best clinical predictor of the procedural end point was the duration of the current AF episode. Univariate differences between the patients with and without AF termination for both cohorts are presented in Tables I and II in the Data Supplement. The cross-validated prediction model with only clinical parameters had a mean area under the curve (AUC) of $0.75 \pm 0.16$ containing the duration of the current AF episode and left atrial diameter. Figure 2 shows an example of lead V1 and the corresponding power spectrum of 2 patients. In 
the patient on the left, AF terminated during the procedure and long-term success of the ablation was achieved. In the patient on the right, neither acute termination nor long-term success could be achieved. Note the higher FWA, higher OI, and lower DF in the patient with success of CA. The ECG parameter providing the best prediction was the FWA. Logistic regression analysis allowing combination of different ECG parameters resulted in a model with a mean AUC of $0.76 \pm 0.15$ containing FWA in lead aVR and the DF in lead aVF. Combining both ECG and clinical predictors revealed a model with a mean AUC of $0.79 \pm 0.13$ containing FWA in lead aVR and the duration of the current AF episode. The predictive value of these models did not differ significantly. The models identified in the training set predicted acute outcome in the validation cohort with an AUC varying from 0.60 to 0.72 . Table 2 provides a complete overview of the models. Interestingly, the model based on ECG parameters had a higher predictive value $(0.72)$ than the one based on the clinical predictors (0.60).

Successful ablation
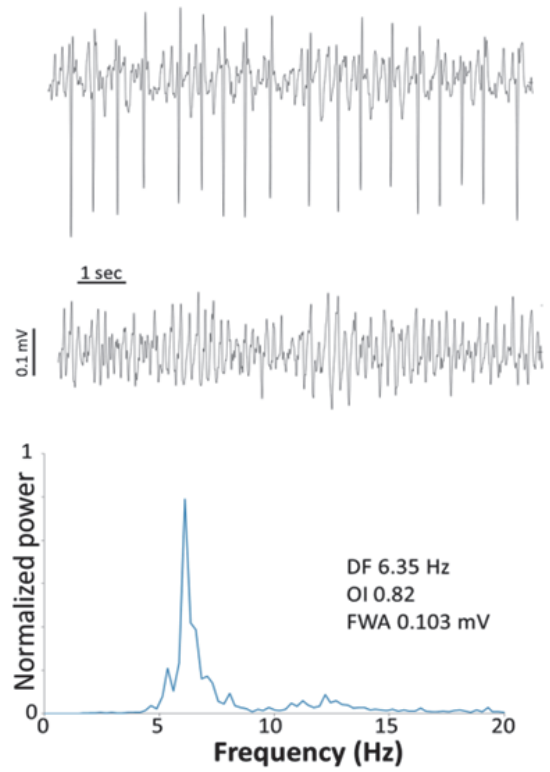

Unsuccessful ablation

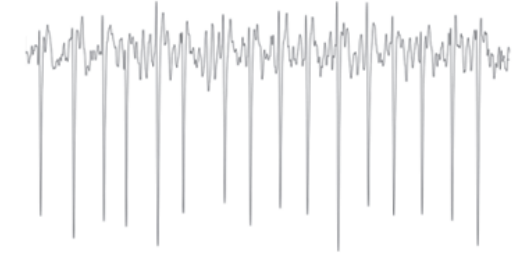

$\underline{1 \mathrm{sec}}$
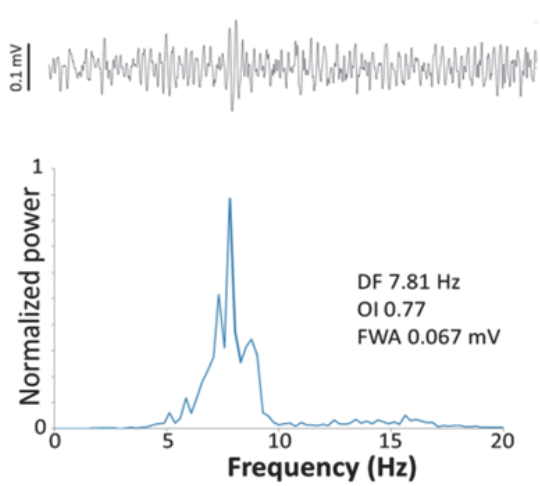

Figure 2: This figure shows lead V1 (upper panel), the extracted atrial signal (middle panel) and the corresponding power spectrum (lower panel) of a patient with success of catheter ablation on the left and a patient with failed catheter ablation on the right. The patient with successful ablation has a lower dominant frequency (DF), higher organization index (OI) and larger F-wave amplitude (FWA) than the patient with unsuccessful ablation. 


\begin{tabular}{|c|c|c|c|}
\hline & $\begin{array}{c}\text { Training set } \\
\text { (Cross validated) AUC }\end{array}$ & $\begin{array}{c}\text { Validation set } \\
\text { AUC }\end{array}$ & Parameters included \\
\hline \multicolumn{4}{|c|}{ Termination during index procedure } \\
\hline Clinical & $0.75 \pm 0.16$ & 0.60 & $\begin{array}{l}\text { Duration current AF } \\
\text { episode, LA diameter }\end{array}$ \\
\hline ECG & $0.76 \pm 0.15$ & 0.72 & FWA aVR, DF aVF \\
\hline Combined & $0.79 \pm 0.13$ & 0.70 & $\begin{array}{l}\text { FWA aVR, Duration } \\
\text { current AF episode }\end{array}$ \\
\hline \multicolumn{4}{|c|}{ Long-term success } \\
\hline Clinical & $0.66 \pm 0.15$ & 0.61 & $\begin{array}{c}\text { Duration current AF } \\
\text { episode }\end{array}$ \\
\hline ECG & $0.69 \pm 0.13$ & 0.63 & FWA V6 \\
\hline Combined & $0.71 \pm 0.12$ & 0.61 & $\begin{array}{l}\text { FWA V6, Duration current } \\
\text { AF episode }\end{array}$ \\
\hline
\end{tabular}

Table 2: Prediction models for the training and validation set on both the acute and long-term endpoint. The prediction models in the training set are cross validated. The cross validated models are tested in the validation set. Atrial fibrillation (AF), dominant frequency (DF), F-wave amplitude (FWA), left atrial (LA).

\begin{tabular}{cccc}
\hline & $\begin{array}{c}\text { Training set } \\
\text { (Cross validated) AUC }\end{array}$ & $\begin{array}{c}\text { Validation set } \\
\text { AUC }\end{array}$ & Parameters included \\
\hline Clinical & $0.71 \pm 0.12$ & 0.73 & AF termination index \\
procedure, Stroke & & FWA V6 \\
ECG & $0.69 \pm 0.13$ & 0.63 & AF termination index \\
Combined & $0.78 \pm 0.12$ & 0.74 & procedure, Stroke, FWA V6 \\
\hline
\end{tabular}

Table 3: Prediction models for long-term outcome including electrophysiological data from the index procedure. The prediction models in the training set are cross validated. The cross validated models are tested in the validation set. Including data from the index procedure improves outcome prediction (table 2). Atrial fibrillation (AF), F-wave amplitude (FWA).

\section{Prediction of long-term arrhythmia outcome}

The univariate differences for the long-term outcome are presented in the supplement tables 3 and 4 . The model including clinical parameters included the duration of the current $A F$ episode and predicted SR after long-term follow-up with a mean AUC $0.66 \pm 0.15$. The model including only ECG 
parameters consisted of the FWA in lead V6 with a mean AUC $0.69 \pm 0.13$. The combination of these parameters predicted long-term SR with a mean AUC $0.71 \pm 0.12$ (not significantly higher). These models predicted long-term SR in the 62 patients in the validation cohort although the predictive value was lower than in the training cohort (table 2). Table 3 represents the prediction models for long term outcome incorporating data from the electrophysiological procedure. Including the procedural endpoint (acute AF termination) in the long-term prediction models improved these models in both the training and validation set.

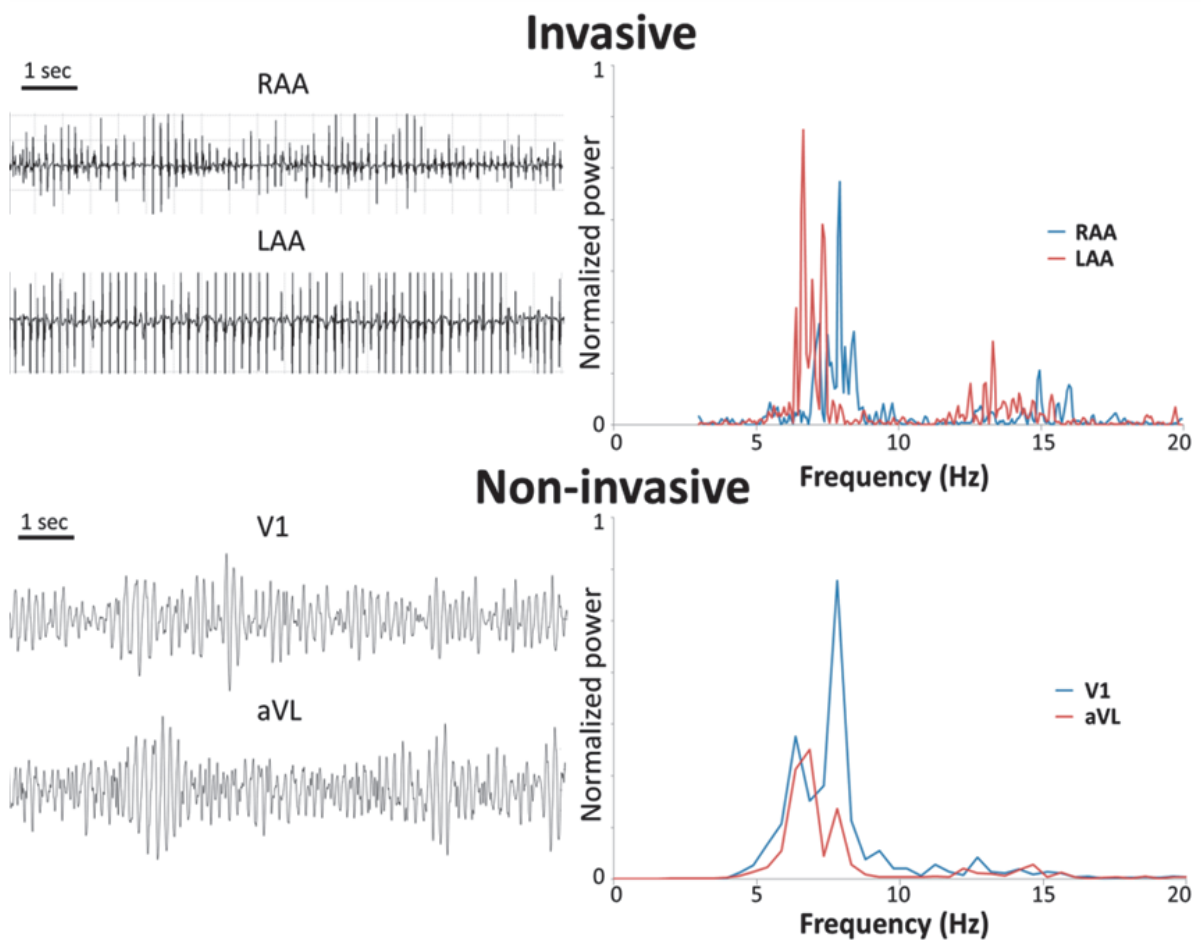

Figure 3: This figure shows an example of a patient with a right to left frequency gradient with simultaneous endocardial recordings in the left and right atrial appendage (LAA and RAA) and surface ECG recordings. The upper part shows the invasive and the lower part the non-invasive recordings. It can be appreciated that the frequency in the RAA (the blue line) corresponds to the frequency measured in lead V1 and that the frequency in the LAA (the red line) corresponds to the frequency in lead aVL.

Correlation between endocardial recordings and corresponding surface leads

To investigate to what extent the ECG parameters measured noninvasively reflect the electrophysiological properties measured invasively, we correlated noninvasive DF with the activation rate in the right and left atria. In 54 patients 


\section{Chapter 6}

of the training cohort, surface ECG recordings could be compared with simultaneous RAA recordings and in 51 patients with LAA recordings. Figure 3 shows an example of the DF in the right and left atria and on the surface leads V1 and aVL. In this patient, the activation frequency was higher in the right atrium than in the left atrium. These frequencies corresponded well to the frequencies in the leads V1 and aVL. Overall, the LAA DF showed a significant correlation with all surface leads, but the correlation was the strongest with lead I $(r=0.733 ; P<0.001)$. In contrast, the RAA DF showed a highly significant correlation with lead V1 $(r=0.870 ; P<0.001)$. The correlation coefficient decreased toward the leftward precordial leads as shown in Figure 4.

\begin{tabular}{lllllllllllll}
\hline & I & II & III & aVR & aVL & aVF & V1 & V2 & V3 & V4 & V5 & V6 \\
RAA & 0.277 & 0.220 & 0.415 & 0.452 & 0.620 & 0.499 & 0.870 & 0.796 & 0.644 & 0.457 & 0.486 & 0.336 \\
LAA & 0.733 & 0.571 & 0.300 & 0.596 & 0.686 & 0.409 & 0.616 & 0.464 & 0.590 & 0.599 & 0.676 & 0.694
\end{tabular}
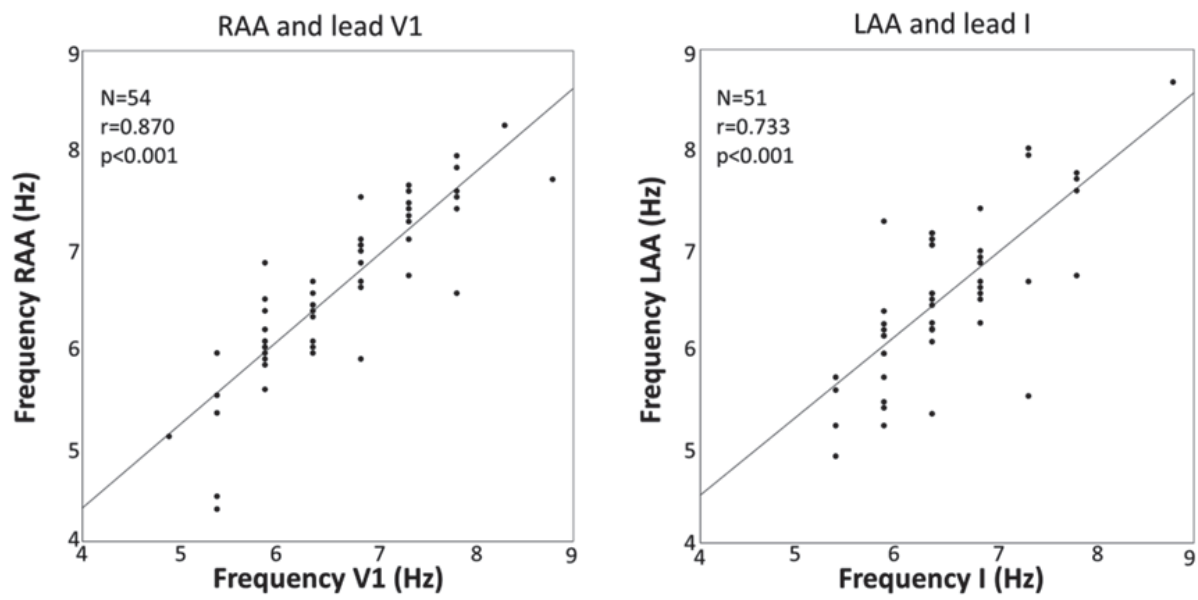

Figure 4: Correlation between the invasively and non-invasively measured dominant frequency (DF). The table shows the Pearson's R for the DF in every lead with the invasive cycle length measured in the right and left atrial appendage (RAA and LAA). The bottom panel shows the best correlation with the right atrial appendage frequency (with lead V1) and with the left atrial appendage (lead I). The frequency measured in the RAA is best resembled by leads V1 and V2. The frequency measured in the LAA corresponds best to the DF measured on leads I, aVL and V6. 
Lead V1

Pre ablation

$1 \mathrm{sec}$

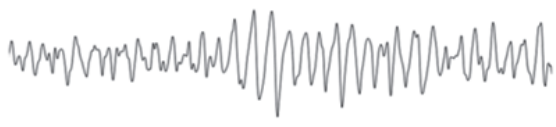

Post PVI

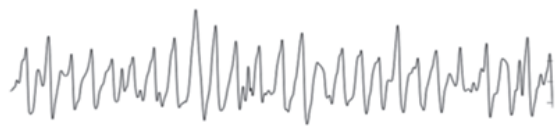

Post LA

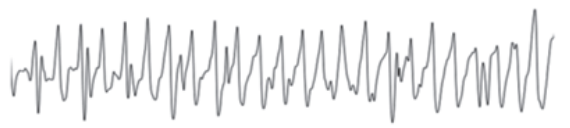

Lead I

Pre ablation

$1 \mathrm{sec}$

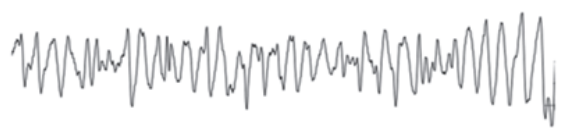

Post PVI

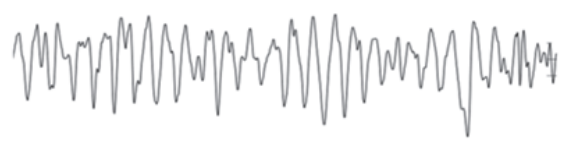

Post LA

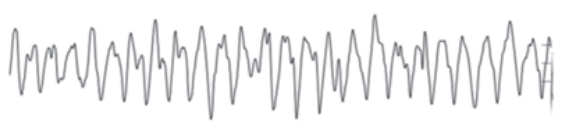

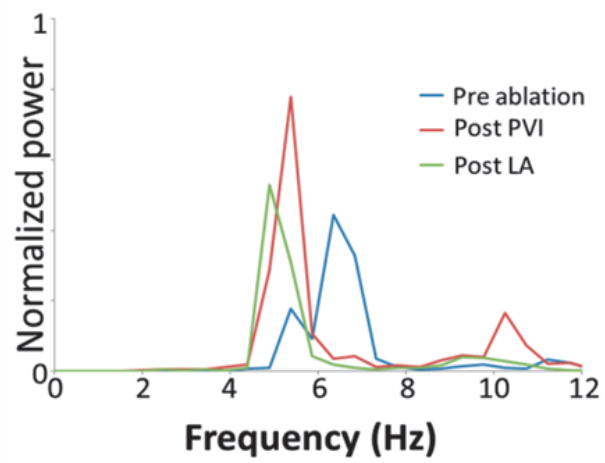

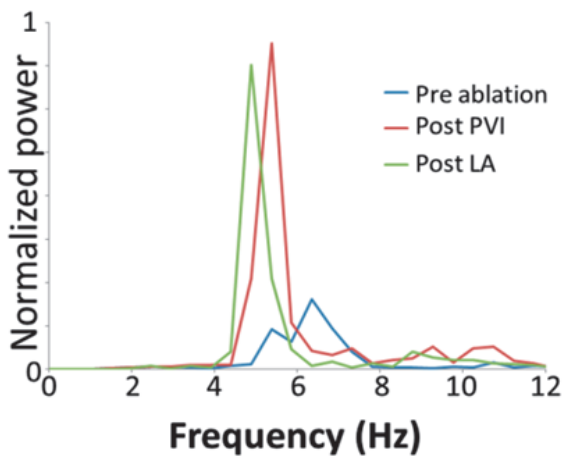

Figure 5: Shown are 5 seconds atrial signals recorded on lead V1 and I of a patient pre ablation and during subsequent ablation steps; post pulmonary vein isolation (PVI) and post left atrial ablation (LA). The atrial signal organises during these steps. The corresponding frequency spectrums show that the highest dominant frequency is present pre ablation and it decreases gradually during the ablation indicated by the blue, red and green lines in the frequency spectrum. 
Chapter 6

\section{Complexity parameters during ablation}

To investigate how local changes of the AF cycle length and AF complexity alter the ECG parameters in various leads, we studied the behaviour of the DF and FWA during CA. In 60 patients of the training cohort, ECGs were available for analysis after every ablation step. If AF terminated during a step, we examined the last ECG before termination. Overall, the DF decreased during every subsequent step. Figure 5 shows a representative example; note that the DF becomes lower in both leads V1 and I. The mean DF of the first ECG of all the patients combined was significantly higher compared with the last ECG $(P<0.001)$ on all leads (Table $V$ in the Data Supplement). Interestingly, not all leads behaved in the same way. In the 11 patients who needed right atrial ablation, overall, the DF in lead V1 decreased significantly during ablation. However, the DF decreased only after pulmonary vein isolation (PVI) and right atrial ablation but not after left-sided ablation (Figure 6). In contrast, the DF in lead V6 decreased during left-sided ablation steps but did not further decrease during right-sided ablation. In most leads, there was a significant difference between the FWA before and at the end of the ablation (Table VI in the Data Supplement). Also in the case of FWA, left and right-sided ablation differently affected the ECG leads. In patients with right atrial ablation, the FWA in lead V1 increased first after PVI and decreased thereafter, although not significantly. FWA in lead V6 decreases significantly during ablation and was affected most by PVI and left atrial ablation ( $P=0.001$; Figure 6).

\section{Discussion}

This study shows that ECG-derived AF complexity parameters predict success of CA in patients with persistent AF. Their predictive performance in the training cohort is at least as good as previously identified clinical predictors. The best non-invasive AF complexity parameter for the prediction of both AF termination and long-term success is the FWA. The results could be successfully validated in an external ablation cohort supporting their general relevance for prediction of outcome in patients undergoing CA. 


\section{Dominant Frequency}

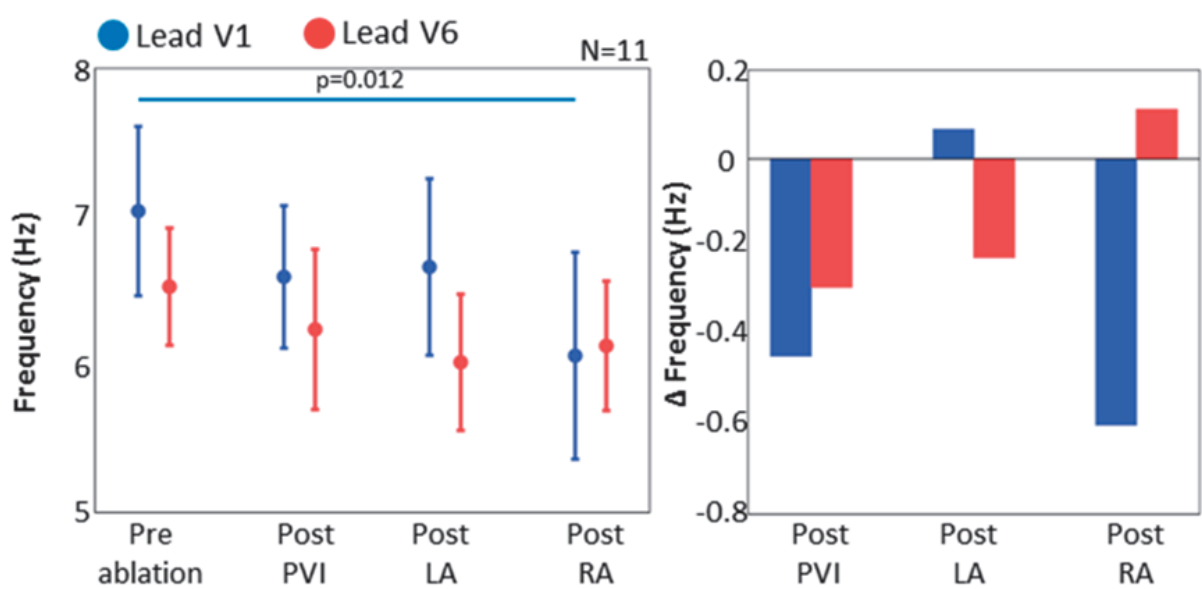

\section{F-wave amplitude}

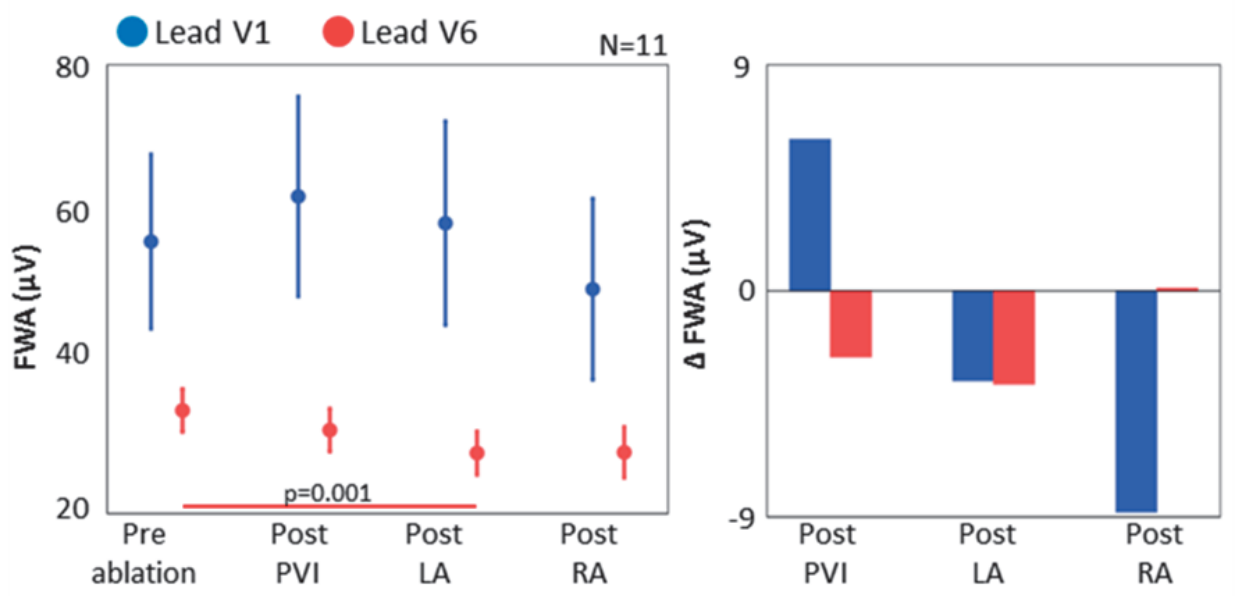

Figure 6: This figure shows left and right differences during ablation. Shown are 11 patients that underwent all ablation steps including right atrial ablation. The graphs on the left show the mean values during ablation and the graphs on the right the mean difference after each step. Left orientated leads were mostly affected by pulmonary vein isolation (PVI), while right orientated leads were predominantly affected by right atrial (RA) ablation. 


\section{Outcome prediction using various ECG AF complexity parameters}

This study shows that a low FWA indicates a less favourable ablation outcome. A low FWA can be caused by an increase in heterogeneity in the atrium, for example, because of a higher number of waves and breakthroughs in patients with complex AF. ${ }^{19}$ This increased heterogeneity leads to more cancellation and hence the lower F-waves. The predictive value of FWA for termination of persistent $A F$ by ablation has been described before. ${ }^{16,20}$ However, these previous studies focussed on lead V1 and did not provide a comparison with other AF complexity parameters or other leads. Furthermore, ECG analysis was done manually. An advantage of automated ECG analysis is that it enables analysis of smaller f-waves in, for instance, lead V6 where manual FWA analysis is hardly possible. Furthermore, automated analysis might prevent a bias potentially introduced by choosing large and easily identifiable F-waves. This also may explain the slightly lower FWAs in our study in comparison to manually analysed F-waves. ${ }^{16,20}$ In line with 1 small previous study, our data show the importance of incorporating all available surface-leads including leftward orientated leads despite their less favourable signal to noise ratio. ${ }^{21}$

We studied a wide variety of time and frequency parameters and their predictive value. DF is the most frequently studied parameter in this respect, and as in our cohort, patients with a lower DF or longer AFCL have a more favourable rhythm outcome. ${ }^{22-24} \mathrm{SE}$ and $\mathrm{OI}$ are calculated based on a larger part of the frequency spectrum than only the DF peak and, therefore in theory, could more reliably reflect disorganised electrical activity. These parameters which measure the regularity or organization of the frequency spectrum, however, appeared to have limited predictive performance. They have been used to predict ablation outcome in a limited number of studies. In 1 study, stability of the frequency spectrum predicted AF termination during the ablation and freedom of atrial arrhythmias after a limited follow-up of 3 months. ${ }^{25}$ SampEn has not been used to predict ablation outcome before. In our study, SampEn showed a low predictive value.

Most importantly, we showed that a model, including AF complexity parameters, not only predicted acute and long-term outcome in 1 specific cohort but also in a persistent AF patient cohort in a different center.

\section{Correlation between invasive and non-invasive parameters for fibrillatory rate}

To confirm that the ECG accurately reflects the electrophysiological properties of the fibrillating atrium, we directly compared simultaneous endocardial and 
ECG recordings. We demonstrate that the DF in the LAA correlates best with leftward leads on a standard ECG, such as lead I, aVL, or V6. Vice versa, DF in RAA correlates best with rightward leads, such as V1. Figure 3 shows results in a patient with a frequency gradient. Both the left and right atrial frequencies can be determined from the surface ECG. The highest peak in lead V1 equals the atrial frequency of the RAA, whereas the DF of aVL reflects the atrial frequency in the LAA. This finding further supports the notion that the electric information in individual leads may reflect the electric properties in different areas of the atria (left versus right), which may be useful for our understanding of the mechanisms of $\mathrm{AF}$ in individual patients. ${ }^{26,27}$

\section{Evolution of the ECG during stepwise ablation}

If the ECG leads reflect activation frequencies in specific regions in the atria, the ECG should respond to local ablation steps, which have been demonstrated to change the atrial fibrillation cycle length nearby the ablation site. Overall, we found a decrease in DF during the ablation. Importantly, the extent of the decrease was clearly related to the location of the ablation. In patients undergoing ablation both in the right and the left atrium, there was a trend toward a larger effect of left atrial ablation on DF in V6, whereas the right atrial ablation had a larger effect on lead V1. This is consistent with a preferential prolongation of atrial fibrillation cycle length in the ablated area.

$\mathrm{F}$ waves are higher in patients in whom AF terminated during ablation, and SR during long-term follow-up was achieved. Therefore, a higher FWA might reflect a more organized AF pattern as discussed before. Because of the increasing organization of AF during ablation, we expected that FWA would increase during the ablation. Surprisingly, this was not the case. There was even a trend to a decrease in FWA. Also, here it is important to note the behaviour of different leads. In lead V1, the FWA showed a slight increase after PVI but decreased during the subsequent ablation steps. The decrease was most pronounced after right-sided ablation. The FWA in lead V1 might have increased after PVI because of abolition of leftward oriented F-wave vectors after PVI, that is, vectors pointing away from V1. In lead V6, there was even a significant decrease in FWA with the largest contribution by left-sided ablation. The decrease in FWA during CA might be because of electric isolation of atrial tissue from the rest of the atrium during the procedure, thereby reducing the atrial mass contributing to the generation of $F$ waves. The hypothesis that the FWA decreases because of the decrease in atrial mass is supported by studies investigating $\mathrm{P}$-wave alterations during $\mathrm{CA}$, with most of these studies 
reporting a shortening of the $\mathrm{P}$-wave duration and decrease in $\mathrm{P}$-wave area after CA as an indicator of a decreased atrial mass. ${ }^{28,29}$

\section{Comparison with clinical predictors}

New predictive markers for outcome are only useful if they outperform existing clinical markers that are easy to assess or if in combination with existing predictors the outcome prediction can be improved. For this reason, we compared the predictive performance of our ECG markers with known clinical parameters of favorable rhythm outcomes, such as a shorter AF duration and a smaller left atrial diameter. ${ }^{4}, 5,7,8,30$ Also, we showed that adding ECG parameters to known clinical parameters improves prediction. The prediction of success of CA using a combination of AF complexity parameters proved to be at least as good as the prediction using known clinical parameters. Although the duration of the current AF episode is an important parameter for outcome prediction, it can be difficult to assess because of the asymptomatic or slightly symptomatic nature of some AF episodes. Our study demonstrates that using objective ECG parameters may serve as a reasonable alternative for outcome prediction in the setting of CA in persistent AF.

\section{Added value of AF termination during index procedure as predictor of long- term outcome}

As shown in Table 2, the predictive value of both clinical and ECG parameters is higher for the prediction of the acute rhythm outcome than that for the longterm outcome. It is not surprising that (electrocardiographic) properties of the present AF episode are related more robustly to acute outcome of the index ablation than to the rhythm 5 years later. Still, the electric complexity before the index ablation predicted success in the long term. Recently, several studies identified termination of AF during the index procedure as a predictor of longterm maintenance of SR, suggesting that terminating AF may serve as an appropriate procedural end point. ${ }^{5,6}$ Indeed, the current data demonstrates that adding information about success of the first procedure substantially improved long-term prediction.

\section{Clinical implications}

Although in paroxysmal AF, success of CA can be obtained in a high percentage of patients, in persistent AF, success rates are lower. The noninvasive atrial complexity parameters identified in this study may provide a useful tool to identify those patients with (long standing) persistent AF who likely benefit from CA and others in whom CA is less likely to be effective. Implementing ECG 
complexity together with other predictive parameters in an individualized stratification model could potentially prevent ablations in patients with a low chance of success during follow-up and thereby avoid unnecessary procedural risks. Alternatively, if a rhythm control strategy is still preferred in patients with a high AF complexity, more aggressive ablation regimens, such as extensive radiofrequency ablation or a hybrid surgical ablation, should be chosen as a first treatment option. ${ }^{31}$ The FWA was identified as the complexity parameter with the highest predictive value for both acute AF termination and long-term success. This parameter is both intuitive and easy to calculate from the ECG leads and could be implemented in clinical practice without large obstacles.

\section{Limitations}

Although this is the largest study so far investigating the predictive value a variety of ECG-derived complexity parameters in a population undergoing CA, the study population was still of limited size. Also, there is some evidence that alternative lead placement provides additional information about the fibrillatory process. However, in this study, we were limited to the standard 12lead ECG and therefore not able to investigate whether a different lead placement improves prediction. For direct comparison between invasive and noninvasive complexity parameters, we were limited to the LAA and RAA measurements. Usually, the appendages have less complex electrograms than other locations in the left and or the right atrium. For this reason, we did not compare invasive complexity parameters, such as fractionation with the noninvasive parameters of complexity. As we used Holter monitoring for rhythm follow-up, we may not have detected some self-terminating episodes of AF. However, no persistent recurrence was documented in patients booked as SR. Also, we did not have specific information about the patients' AAD therapies other than amiodarone. AADs and upstream therapy medication may influence the atrial remodeling process and suppress recurrences and thereby have affected our prediction models. Furthermore, we did not include the possible predictive value of blood biomarkers in our study. There is evidence that elevated cardiac biomarkers, such as B-type natriuretic peptide or markers of inflammation, predict ablation outcome. ${ }^{32,}{ }^{33}$ Also, the possible predictive value of single nucleotide variants as shown before was not included in this study. ${ }^{34}$ Follow-up data in the validation cohort were available in only $77 \%$ of the patients. However, baseline characteristics did not differ between those patients and patients with complete follow-up. We were unable to study the relationship between ECG complexity parameters and effects of different sets of ablation lesions because the latter was not randomized that precluded an 
Chapter 6

unbiased assessment. Finally, data from follow-up ablations are useful to analyze the mechanisms of arrhythmia recurrence and to correlate these mechanisms to AF complexity. However, data on follow-up ablations was too limited. Future research should address these research questions.

\section{Conclusions}

ECG-derived complexity parameters provide reliable information on the electrophysiological properties of the fibrillating atrium and can predict both acute termination of AF during CA and success of rhythm control during longterm follow-up. The predictive performance of ECG-derived parameters is at least as good as known clinical predictive parameters. Complexity analysis of the standard 12-channel ECG may help to identify patients in whom CA is likely to be effective and also those patients in whom SR cannot be maintained even after several CA procedures. 


\section{References}

1. Terasawa T, Balk EM, Chung M, Garlitski AC, Alsheikh-Ali AA, Lau J, Ip S. Systematic review: Comparative effectiveness of radiofrequency catheter ablation for atrial fibrillation. Ann Intern Med. 2009;151:191-202.

2. Wynn GJ, Das M, Bonnett LJ, Panikker S, Wong T, Gupta D. Efficacy of catheter ablation for persistent atrial fibrillation: A systematic review and metaanalysis of evidence from randomised and non-randomised controlled trials. Circ Arrhythm Electrophysiol. 2014;7:841-852.

3. Tilz RR, Rillig A, Thum AM, Arya A, Wohlmuth P, Metzner A, Mathew S, Yoshiga $\mathrm{Y}$, Wissner E, Kuck KH, Ouyang F. Catheter ablation of long-standing persistent atrial fibrillation: 5-year outcomes of the hamburg sequential ablation strategy. J Am Coll Cardiol. 2012;60:1921-1929.

4. Rostock T, Salukhe TV, Steven D, Drewitz I, Hoffmann BA, Bock K, Servatius H, Mullerleile K, Sultan A, Gosau N, Meinertz T, Wegscheider K, Willems S. Longterm single- and multiple-procedure outcome and predictors of success after catheter ablation for persistent atrial fibrillation. Heart Rhythm. 2011;8:13911397.

5. Scherr D, Khairy P, Miyazaki S, Aurillac-Lavignolle V, Pascale P, Wilton SB, Ramoul K, Komatsu Y, Roten L, Jadidi A, Linton N, Pedersen M, Daly M, O'Neill $M$, Knecht S, Weerasooriya R, Rostock T, Manninger M, Cochet $H$, Shah AJ, Yeim S, Denis A, Derval N, Hocini M, Sacher F, Haissaguerre M, Jais P. Fiveyear outcome of catheter ablation of persistent atrial fibrillation using termination of atrial fibrillation as a procedural endpoint. Circ Arrhythm Electrophysiol. 2015;8:18-24.

6. Schreiber D, Rostock T, Frohlich M, Sultan A, Servatius H, Hoffmann BA, Luker J, Berner I, Schaffer B, Wegscheider K, Lezius S, Willems S, Steven D. Five-year follow-up after catheter ablation of persistent atrial fibrillation using the stepwise approach and prognostic factors for success. Circ Arrhythm Electrophysiol. 2015;8:308-317.

7. Takahashi Y, Takahashi A, Kuwahara T, Fujino T, Okubo K, Kusa S, Fujii A, Yagishita A, Miyazaki S, Nozato T, Hikita H, Hirao K, Isobe M. Clinical characteristics of patients with persistent atrial fibrillation successfully treated by left atrial ablation. Circ Arrhythm Electrophysiol. 2010;3:465-471.

8. Zhuang J, Wang Y, Tang K, Li X, Peng W, Liang C, Xu Y. Association between left atrial size and atrial fibrillation recurrence after single circumferential pulmonary vein isolation: A systematic review and meta-analysis of observational studies. Europace. 2012;14:638-645.

9. Lankveld TA, Zeemering S, Crijns HJ, Schotten U. The ecg as a tool to determine atrial fibrillation complexity. Heart. 2014;100:1077-1084.

10. Schotten U, Verheule S, Kirchhof P, Goette A. Pathophysiological mechanisms of atrial fibrillation: A translational appraisal. Physiol Rev. 2011;91:265-325.

11. O'Neill MD, Jais P, Takahashi $Y$, Jonsson A, Sacher F, Hocini M, Sanders $P$, Rostock T, Rotter M, Pernat A, Clementy J, Haissaguerre M. The stepwise 
ablation approach for chronic atrial fibrillation--evidence for a cumulative effect. J Interv Card Electrophysiol. 2006;16:153-167.

12. Rostock T, Steven D, Hoffmann B, Servatius H, Drewitz I, Sydow K, Mullerleile K, Ventura R, Wegscheider K, Meinertz T, Willems S. Chronic atrial fibrillation is a biatrial arrhythmia: Data from catheter ablation of chronic atrial fibrillation aiming arrhythmia termination using a sequential ablation approach. Circ Arrhythm Electrophysiol. 2008;1:344-353.

13. Alcaraz R, Hornero F, Rieta JJ. Assessment of non-invasive time and frequency atrial fibrillation organization markers with unipolar atrial electrograms. Physiol Maes. 2011;32:99-114.

14. Chiarugi F, Varanini M, Cantini F, Conforti F, Vrouchos G. Noninvasive ecg as a tool for predicting termination of paroxysmal atrial fibrillation. IEEE Trans Biomed Eng. 2007;54:1399-1406.

15. Uldry L, Van Zaen J, Prudat Y, Kappenberger L, Vesin JM. Measures of spatiotemporal organization differentiate persistent from long-standing atrial fibrillation. Europace. 2012;14:1125-1131.

16. Nault I, Lellouche N, Matsuo S, Knecht S, Wright M, Lim KT, Sacher F, Platonov P, Deplagne A, Bordachar P, Derval N, O'Neill MD, Klein GJ, Hocini M, Jais P, Clementy J, Haissaguerre $\mathrm{M}$. Clinical value of fibrillatory wave amplitude on surface ecg in patients with persistent atrial fibrillation. J Interv Card Electrophysiol. 2009;26:11-19.

17. Alcaraz R, Abasolo D, Hornero R, Rieta JJ. Optimal parameters study for sample entropy-based atrial fibrillation organization analysis. Comput Methods Programs Biomed. 2010;99:124-132.

18. $\mathrm{Ng} \mathrm{J}$, Kadish AH, Goldberger JJ. Technical considerations for dominant frequency analysis. J Cardiovasc Electrophysiol. 2007;18:757-764.

19. de Groot NM, Houben RP, Smeets JL, Boersma E, Schotten U, Schalij MJ, Crijns $\mathrm{H}$, Allessie MA. Electropathological substrate of longstanding persistent atrial fibrillation in patients with structural heart disease: Epicardial breakthrough. Circulation. 2010;122:1674-1682.

20. Cheng Z, Deng H, Cheng K, Chen T, Gao P, Yu M, Fang Q. The amplitude of fibrillatory waves on leads avf and $v 1$ predicting the recurrence of persistent atrial fibrillation patients who underwent catheter ablation. Ann Noninvasive Electrocardiol. 2013;18:352-358.

21. Meo M, Zarzoso V, Meste O, Latcu DG, Saoudi N. Spatial variability of the 12lead surface ecg as a tool for noninvasive prediction of catheter ablation outcome in persistent atrial fibrillation. IEEE Trans Biomed Eng. 2013;60:2027.

22. Matsuo $S$, Lellouche $N$, Wright $M$, Bevilacqua $M$, Knecht $S$, Nault I, Lim KT, Arantes L, O'Neill MD, Platonov PG, Carlson J, Sacher F, Hocini M, Jais P, Haissaguerre $M$. Clinical predictors of termination and clinical outcome of catheter ablation for persistent atrial fibrillation. J Am Coll Cardiol. 2009;54:788-795.

23. Drewitz I, Willems S, Salukhe TV, Steven D, Hoffmann BA, Servatius H, Bock K, Aydin MA, Wegscheider K, Meinertz T, Rostock T. Atrial fibrillation cycle 
length is a sole independent predictor of a substrate for consecutive arrhythmias in patients with persistent atrial fibrillation. Circ Arrhythm Electrophysiol. 2010;3:351-360.

24. Haissaguerre $M$, Lim KT, Jacquemet V, Rotter M, Dang L, Hocini M, Matsuo S, Knecht $S$, Jais P, Virag N. Atrial fibrillatory cycle length: Computer simulation and potential clinical importance. Europace. 2007;9 Suppl 6:vi64-70.

25. Di Marco LY, Raine D, Bourke JP, Langley P. Characteristics of atrial fibrillation cycle length predict restoration of sinus rhythm by catheter ablation. Heart Rhythm. 2013;10:1303-1310.

26. Guillem MS, Climent AM, Millet J, Arenal A, Fernandez-Aviles F, Jalife J, Atienza F, Berenfeld O. Noninvasive localization of maximal frequency sites of atrial fibrillation by body surface potential mapping. Circ Arrhythm Electrophysiol. 2013;6:294-301.

27. Bollmann A, Husser D, Mainardi L, Lombardi F, Langley P, Murray A, Rieta JJ, Millet J, Olsson SB, Stridh M, Sornmo L. Analysis of surface electrocardiograms in atrial fibrillation: Techniques, research, and clinical applications. Europace. 2006;8:911-926.

28. Van Beeumen K, Houben R, Tavernier R, Ketels S, Duytschaever M. Changes in $\mathrm{p}$-wave area and $\mathrm{p}$-wave duration after circumferential pulmonary vein isolation. Europace. 2010;12:798-804.

29. Maan A, Mansour M, Ruskin JN, Heist EK. Impact of catheter ablation on $\mathrm{p}$ wave parameters on 12-lead electrocardiogram in patients with atrial fibrillation. J Electrocardiol. 2014;47:725-733.

30. O'Neill MD, Wright $M$, Knecht $S$, Jais $P$, Hocini $M$, Takahashi $Y$, Jonsson $A$, Sacher F, Matsuo S, Lim KT, Arantes L, Derval N, Lellouche N, Nault I, Bordachar P, Clementy J, Haissaguerre M. Long-term follow-up of persistent atrial fibrillation ablation using termination as a procedural endpoint. Eur Heart J. 2009;30:1105-1112.

31. Pison L, La Meir M, van Opstal J, Blaauw Y, Maessen J, Crijns HJ. Hybrid thoracoscopic surgical and transvenous catheter ablation of atrial fibrillation. $J$ Am Coll Cardiol. 2012;60:54-61.

32. Hussein AA, Saliba WI, Martin DO, Shadman M, Kanj M, Bhargava M, Dresing T, Chung M, Callahan T, Baranowski B, Tchou P, Lindsay BD, Natale A, Wazni OM. Plasma b-type natriuretic peptide levels and recurrent arrhythmia after successful ablation of lone atrial fibrillation. Circulation. 2011;123:2077-2082.

33. Wu N, Xu B, Xiang Y, Wu L, Zhang Y, Ma X, Tong S, Shu M, Song Z, Li Y, Zhong L. Association of inflammatory factors with occurrence and recurrence of atrial fibrillation: A meta-analysis. Int J Cardiol. 2013;169:62-72.

34. Husser D, Adams V, Piorkowski C, Hindricks G, Bollmann A. Chromosome 4q25 variants and atrial fibrillation recurrence after catheter ablation. J Am Coll Cardiol. 2010;55:747-753. 


\section{Part III}

\section{ECG derived}

parameters in

clinical

context 


\section{Chapter 7}

Clinical correlates of noninvasive ECG parameters in patients with persistent atrial fibrillation

Theo Lankveld, Stef Zeemering, Boris A Hoffmann, Pierre Jaïs, Harry J Crijns and Ulrich Schotten 
Chapter 7

\section{Abstract}

Background: Non-invasive ECG-derived atrial complexity and frequency parameters are promising parameters to guide individualised atrial fibrillation (AF) treatment. However, clinical comorbidities might significantly affect these parameters.

Methods: We analysed the dominant frequency (DF) and F-wave amplitude (FWA) on digitally recorded 10 seconds 12 lead ECGs of patients with persistent AF. We correlated these ECG-derived parameters with clinical and echocardiographic parameters known to predict rhythm outcome.

Results: We included 754 patients (mean age $65 \pm 11$ years, 73\% male). Antiarrhythmic drug treatment significantly lowers the DF $(P<0.001)$. Also cardiovascular co-morbidities and advancing age all are associated with a lower DF. Women have a lower DF than men. Anti-arrhythmic drugs, advancing age and female gender were independently associated with a lower DF. The FWA decreases with increasing duration of the episode. The FWA is higher in women than in men in most leads. Cardiovascular co-morbidities tend to lower the FWA. A longer duration of the current episode was independently associated with a lower FWA.

Conclusions: Clinical parameters independently influence ECG-derived atrial frequency and complexity parameters but do not fully explain variability in ECG parameters. These data support the rationale for the incorporation of both ECG and clinical parameters in future models on individualised AF treatment. 


\section{Introduction}

There are several treatment strategies to pursue rhythm control in patients with atrial fibrillation (AF), for example electrical cardioversion, catheter or surgical ablation all with or without anti-arrhythmic drugs. ${ }^{1}$ Regardless of the strategy chosen early treatment seems most effective for maintaining sinus rhythm. Furthermore, AF is a multifactorial disease with a large variability in signalling pathways resulting in structural remodelling processes and electrophysiological mechanisms triggering or perpetuating the arrhythmia. Therefore, it is worthwhile to select the appropriate treatment for the individual patient in an early stage.

Numerous studies have been undertaken to predict success of a rhythm control strategy in these patients. Research focussed on clinical, echocardiographic, blood biomarkers, genetics, the electrocardiogram (ECG) or a combination of different modalities. The ECG is available in every patient with $\mathrm{AF}$ and therefore is an interesting modality for researchers. Many parameters have been identified to characterise the complexity of the fibrillatory pattern using an ECG. Based on recent publications that systematically analysed a variety of time- and frequency-domain parameters, the most suitable candidates to use in clinical practice seem to be the dominant frequency (DF) and the F-wave amplitude (FWA). ${ }^{2,3}$ Although most studies showed a favourable rhythm outcome for patients with a low DF and a high FWA, results are not consistent throughout all studies. ${ }^{4}$ In part these differences might be related to the different populations studied. In this study we investigate the influence of clinical characteristics on the DF and FWA in a large cohort of patients with persistent AF.

\section{Methods}

\section{Patient population}

We studied patients with persistent AF from different cohorts. We included patients admitted for electrical cardioversion at the Maastricht University Medical Centre, Maastricht, The Netherlands, and patients with de novo stepwise catheter ablation between July 2004 and October 2007 at the Hôpital Cardiologique du Haut Lévêque, Bordeaux, France or between December 2006 and May 2009 at the University Hospital Eppendorf, Hamburg, Germany. Patients without a digitally recorded 10 seconds 12 lead ECG were excluded. Clinical parameters and echocardiographic parameters were retrieved from the medical charts. The analysis was performed retrospectively. 


\section{ECG analysis}

The ECGs were analysed as documented in more detail previously. ${ }^{2,3}$ Briefly, we analysed digitally recorded and exported 10 seconds 12 lead ECG. ECGs were exported from the BARD LabSystem pro mapping system (LabSystem ${ }^{\mathrm{TM}}$ PRO, Bard EP, Lowell, MA, USA) in patients that underwent catheter ablation and from the GE MAC 5500 ECG recording device in patients included the Maastricht University Medical Centre. After filtering with a $1-100 \mathrm{~Hz}$ bandpass filter QRST cancellation was applied. Before the analysis an additional $3 \mathrm{~Hz}$ high-pass filter was applied to reduce influence of the possible residual T-wave. The DF was defined as the highest peak in the frequency spectrum. This frequency spectrum was constructed using a Fast Fourier Transform. The FWA is the mean amplitude of the F-waves during the 10 seconds. Voltage fluctuations were considered an F-wave if the deflection crossed the baseline and with a minimal interval of $100 \mathrm{~ms}$ between peaks.

\section{Statistical analysis}

Statistical analysis was performed using SPSS statistical software (IBM SPSS statistics version 21.0). Categorical variables are reported as number of patients and percentage. Continuous variables are presented as mean \pm standard deviation or as median with interquartile range depending on their normal distribution. Differences between continuous variables were compared using the Student's t-test or the Mann-Whitney $U$ test in not normally distributed data. Correlations were tested using Pearson's correlation. Multivariable analysis was performed using backward linear regression analysis. Univariate significant parameters were included in the multivariable analysis. A P-value $<0.05$ was considered statistically significant.

\section{Results}

\section{Study population}

We included 754 patients with persistent AF in this study with a mean age $65 \pm$ 11 years, $73 \%$ of the patients was male. The patients' characteristics are shown in table 1. 


\begin{tabular}{lc}
\hline & Patient population (N=754) \\
\hline Age (y) & $65 \pm 11$ \\
Male & $550(73 \%)$ \\
BMI & $28.6 \pm 5.1$ \\
Hypertension & $441(58 \%)$ \\
Diabetes Mellitus & $82(11 \%)$ \\
Coronary artery disease & $182(24 \%)$ \\
AF-duration (months) & $10 \pm 18$ \\
Anti-arrhythmic drugs & $186(25 \%)$ \\
LVEF (\%) & $52 \pm 13$ \\
Left atrial diameter (mm) & $46 \pm 7$ \\
\hline
\end{tabular}

Table 1: Baseline characteristics. Atrial fibrillation (AF), body mass index (BMI), left ventricular ejection fraction (LVEF).

\section{Dominant frequency}

The univariate results are represented in table 2. The DF was significantly lower in patients treated with AADs $(P<0.001)$ on all leads. The DF was lower in women compared to men in almost all leads. Furthermore, cardiovascular diseases such as hypertension, coronary artery disease and diabetes mellitus all were associated with a lower DF. There was a weak but significant negative correlation between increasing age and the DF $(R=-0.115$ to $-0.228 ; P<0.001$ in all leads). Surprisingly, we found no linear or quadratic correlation between the duration of the AF episode and the DF. We also found no correlation between the left ventricular ejection fraction or left atrial diameter with the DF. Table 3 shows all parameters independently associated with a lower DF on every lead with the most important parameter listed first. The most important parameters influencing the DF are AAD treatment, age and gender.

\section{F-wave amplitude}

A longer duration of the AF episode was weak but significantly associated with a lower FWA ( $\mathrm{R}=-0.160$ to -0.223 ; $\mathrm{P}<0.001$ on standard leads). Furthermore, the FWA was lower in patients with hypertension, coronary artery disease, diabetes mellitus and decreased with increasing age (tables 4 and 5). For gender, the results were less consistent. In the limb leads female gender is associated with a higher FWA but in the precordial leads with a lower FWA (leads V2-V4). After multivariate correction, the episode 
Chapter 7

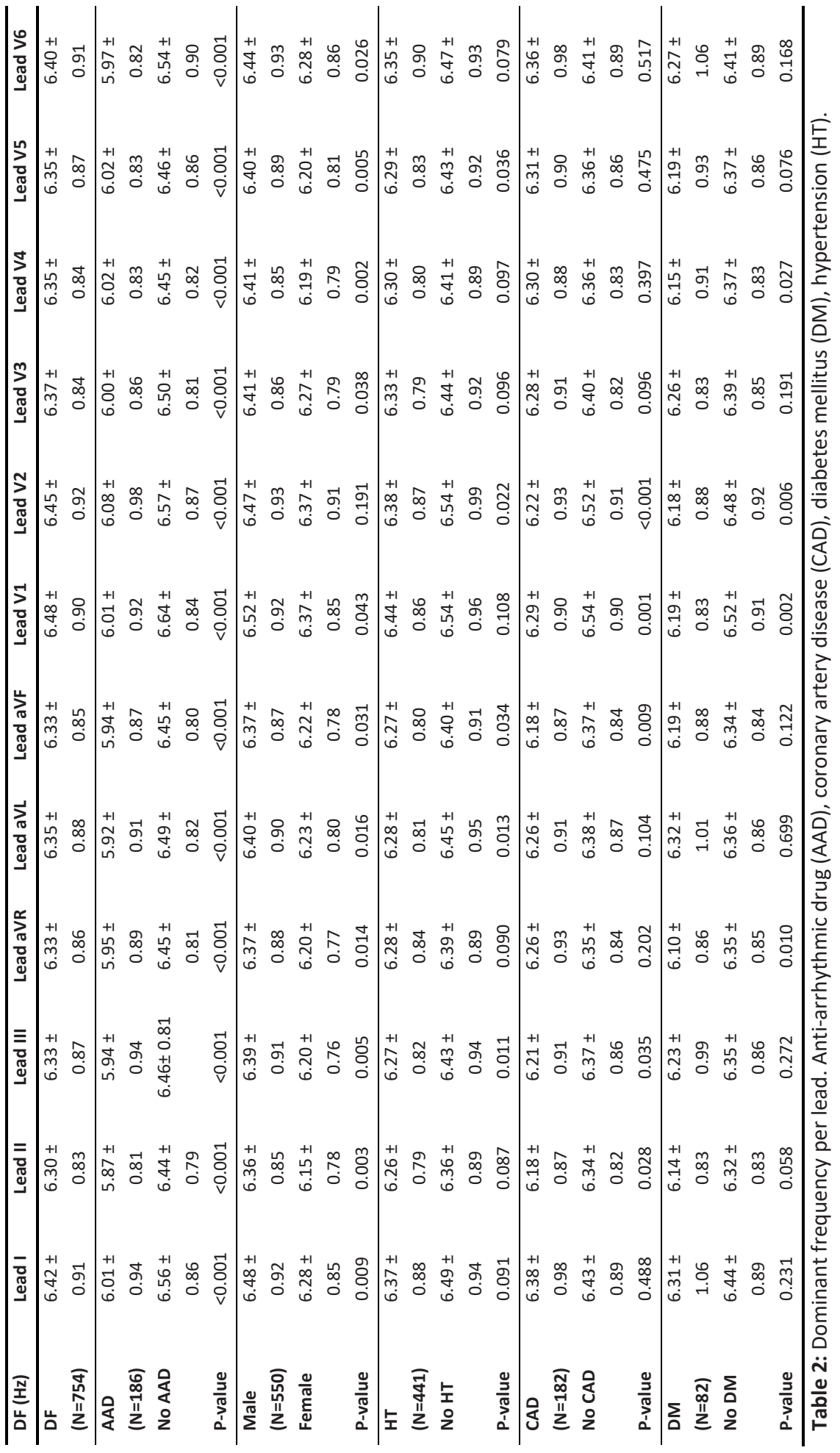


duration seems to be the most important independently associated parameter with a lower FWA (table 5).

\section{Discussion}

This is the largest study so far on the association between clinical characteristics and the electrophysiological characteristics of atrial fibrillation determined from the non-invasive ECG parameters DF and FWA.

\section{Dominant frequency}

The DF is a surrogate parameter for the atrial fibrillation cycle length and is determined by the atrial refractory period and the excitable gap. Not surprisingly, the most important parameter influencing the DF is the use of AADs (in the present study mostly Amiodarone). Amiodarone prolongs the refractory period, slows down conduction and thereby prolongs the AF cycle length. A second contributor to a lower DF is advancing age as observed in previous studies. $^{5-7}$ There have been studies showing that in patients with enhanced atrial fibrosis the atrial fibrillatory rate tends to be lower. ${ }^{8,9}$

Our study indicates that patients with cardiovascular comorbidities like hypertension, coronary artery disease or diabetes have a lower DF. Most of these co-morbidities promote atrial structural remodelling. In a sheep model of hypertension, atrial conduction velocity was reduced, atrial fibrosis was more pronounced and AF susceptibility was enhanced. ${ }^{10}$ Also diabetes mellitus has been linked to atrial fibrosis, ${ }^{11}$ in patients with diabetes mellitus epicardial adipose tissue shows a marked fibrotic effect. ${ }^{12}$ This might explain the association between cardiovascular co-morbidities and a decrease in atrial frequency. In contrast to other studies we did not find a correlation between the duration of the AF episode and the DF. ${ }^{13}$ In many patients with persistent AF it can be difficult to robustly assess the start of the episode due to lack of symptoms. In the present study we assessed the onset of the AF episode based on hospital charts as accurately as possible. However, by excluding patients with a current AF episode duration $>1$ year we found a weak but significant correlation between a higher DF and longer AF episode duration. This can possibly be explained by the relatively stable DF in patients with long-standing persistent AF. ${ }^{4}$

\section{F-wave amplitude}

The amplitude of electrocardiographic signals on the body surface is determined by amplitude of the activation vector, the conductance properties 


\section{Chapter 7}

of the body and the location of the electrode relative to the vector. The amplitude of the vector is determined by the activated atrial mass generating the F-waves, the uniformity of the path the activation follows and the conduction velocity of the generating fibrillation wavefront. In case of AF of low complexity only few but large atrial waves are present creating strong vectors generating relatively large F-waves. During complex atrial fibrillation with a more disorganised pattern more simultaneous waves are present, resulting in a smaller vector and thus lower F-waves. The latter might explain the lower F-waves in patients less likely to benefit from catheter ablation., 14

The most important predictor of a decrease in FWA was a longer duration of the current AF episode. Atrial fibrillation itself promotes the stability of the atrial fibrillation process. ${ }^{15}$ Electrical remodelling with a shortening of the action potential mainly occurs within the first days. ${ }^{15}$ After the initial electrical remodelling, structural remodelling further increases the stability of AF. This increase in stability is accompanied by an increase in the number of atrial breakthroughs, and waves, and conduction block caused by progressive fibrosis of the atrial wall. ${ }^{16,17}$ This increase in complexity of AF with a decrease in simultaneously activated atrial myocardium could explain the decrease in FWA measured with an increase in duration of the AF episode. As mentioned before, robust assessment of the duration of the current AF episode can be difficult and this uncertainty may have confounded our results.

The cardiovascular co-morbidities examined in the present study all seem to lower the F-wave amplitude. As mentioned before these co-morbidities all promote structural remodelling of the atria which promotes a more disorganised AF pattern. This results in a lower atrial vector and lower F-waves on the body surface.

Voltage criteria for left ventricular hypertrophy are less reliable in obese patients due to a decrease in amplitude in these patients. ${ }^{18}$ This can be explained by the longer electrode heart distance as a result of adipose tissue. This longer electrode heart distance may well have contributed to the low FWA in the precordial leads in patients with a higher BMI.

\section{Gender differences}

We showed that women have a lower DF then men. This lower DF in women has been shown before in some cohorts. ${ }^{5,6}$ However, the pathophysiological explanation for this difference in atrial frequency is not completely understood. The combination of a higher FWA and lower DF in women could be interpreted as a less complex fibrillatory pattern in women. A recent study 
however found no systematic gender differences in human pulmonary vein or left atrial electrophysiology in patients with or without AF. ${ }^{19}$

A recent publication demonstrated a higher degree of atrial fibrosis quantified by delayed enhanced magnetic resonance imaging in female patients. ${ }^{20}$ The fact that atrial fibrosis is more pronounced in women may have contributed to the lower DF in females in our cohort. The higher F-waves in women in the standard leads seem contradictory to the observation that women have more pronounced fibrosis. However, this increase in atrial fibrosis seems to be confined to the left atrial posterior wall. ${ }^{20}$ This in turn could lead to less cancellation of atrial activation and therefore a larger vector resulting in larger F-waves in the opposite direction.

The largest difference in DF between men and women can be observed in patients aged $<65$ years. This could be explained by hormonal influences in premenopausal women with for example higher oestrogen levels in premenopausal women. An animal-study showed that dogs receiving $17 \beta-$ estradiol had prolonged atrial effective refractory periods during atrial tachycardia. ${ }^{21}$ The prolonged atrial effective refractory period prolongs the atrial fibrillation cycle length and thus lowers the DF.

\section{Limitations}

We tried to estimate the duration of the AF episode as robustly as possible. Due to the retrospective nature of the study and asymptomatic episodes in some patients we might have underestimated the duration in some patients.

\section{Conclusions}

Anti-arrhythmic drug treatment, advancing age and female gender are the most important clinical parameters independently associated with a lower dominant frequency of F-waves in the ECG. The duration of the AF episode is the most significant independent parameter associated with a lower F-wave amplitude. Better understanding of how AF characteristics based on the ECG are related to co-morbidities, age and gender may help to advance our understanding on how these conditions affect the mechanism, the fibrillatory rate and the complexity of AF. 
Chapter 7

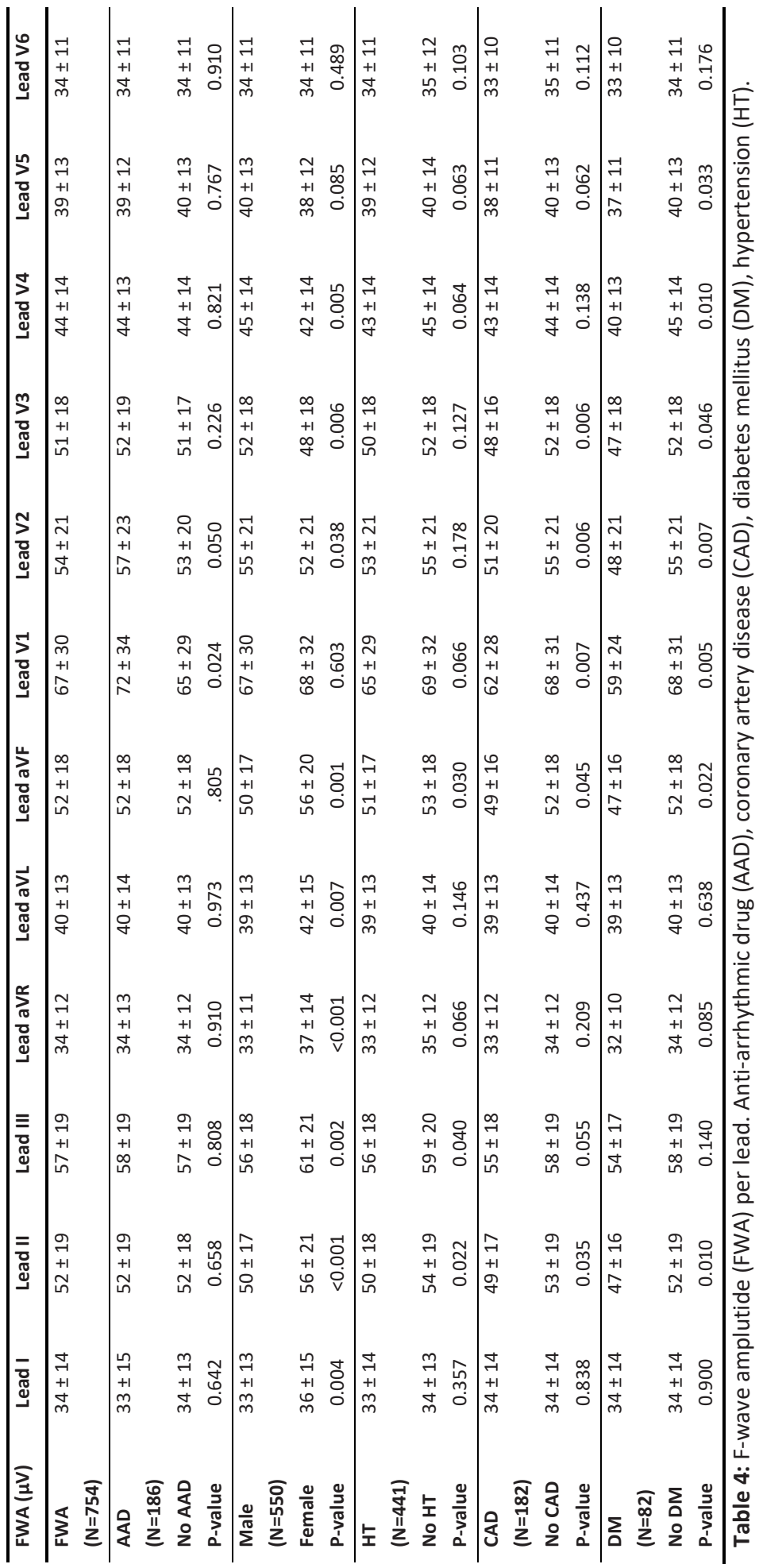


Clinical correlates

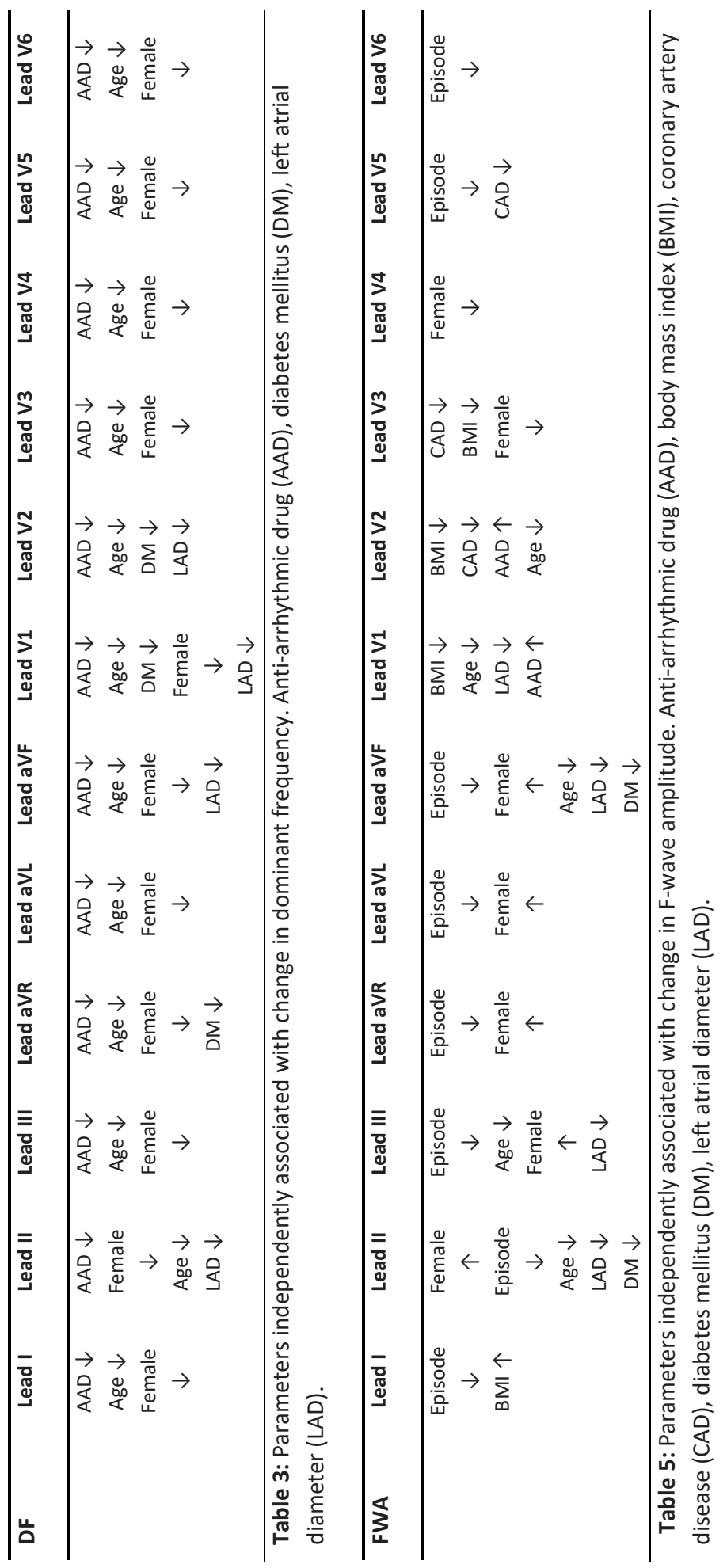




\section{Chapter 7}

\section{References}

1. Camm AJ, Kirchhof P, Lip GY, et al. Guidelines for the management of atrial fibrillation: the Task Force for the Management of Atrial Fibrillation of the European Society of Cardiology (ESC). European Heart Journal 2010;31:23692429.

2. Lankveld T, de Vos CB, Limantoro I, Zeemering S, Dudink E, Crijns HJ, Schotten U. Systematic Analysis of ECG Predictors for Sinus Rhythm Maintenance Following Electrical Cardioversion for Persistent Atrial Fibrillation. Heart rhythm 2016;13:1020-7.

3. Lankveld T, Zeemering S, Scherr D, Kuklik P, Hoffmann BA, Willems S, Pieske B, Haissaguerre M, Jais P, Crijns HJ, Schotten U. Atrial Fibrillation Complexity Parameters Derived From Surface ECGs Predict Procedural Outcome and Long-Term Follow-Up of Stepwise Catheter Ablation for Atrial Fibrillation. Circulation Arrhythmia and Electrophysiology 2016;9:e003354.

4. Platonov PG, Corino VD, Seifert M, Holmqvist F, Sornmo L. Atrial fibrillatory rate in the clinical context: natural course and prediction of intervention outcome. Europace 2014;16 Suppl 4:iv110-iv119.

5. Choudhary MB, Holmqvist F, Carlson J, Nilsson HJ, Roijer A, Platonov PG. Low atrial fibrillatory rate is associated with spontaneous conversion of recentonset atrial fibrillation. Europace 2013;15:1445-1452.

6. Bollmann A, Tveit A, Husser D, Stridh M, Sornmo L, Smith P, Olsson SB. Fibrillatory rate response to candesartan in persistent atrial fibrillation. Europace 2008;10:1138-1144.

7. Xi Q, Sahakian AV, Frohlich TG, Ng J, Swiryn S. Relationship between pattern of occurrence of atrial fibrillation and surface electrocardiographic fibrillatory wave characteristics. Heart Rhythm 2004;1:656-663.

8. Koduri H, Ng J, Cokic I, Aistrup GL, Gordon D, Wasserstrom JA, Kadish AH, Lee R, Passman R, Knight BP, Goldberger JJ, Arora R. Contribution of fibrosis and the autonomic nervous system to atrial fibrillation electrograms in heart failure. Circulation Arrhythmia and Electrophysiology 2012;5:640-649.

9. Swartz MF, Fink GW, Lutz CJ, Taffet SM, Berenfeld O, Vikstrom KL, Kasprowicz $\mathrm{K}$, Bhatta L, Puskas F, Kalifa J, Jalife J. Left versus right atrial difference in dominant frequency, $\mathrm{K}(+)$ channel transcripts, and fibrosis in patients developing atrial fibrillation after cardiac surgery. Heart Rhythm 2009;6:14151422.

10. Kistler PM, Sanders P, Dodic M, Spence SJ, Samuel CS, Zhao C, Charles JA, Edwards GA, Kalman JM. Atrial electrical and structural abnormalities in an ovine model of chronic blood pressure elevation after prenatal corticosteroid exposure: implications for development of atrial fibrillation. European Heart Journal 2006;27:3045-3056.

11. Linz D, Hohl M, Dhein S, Ruf S, Reil JC, Kabiri M, Wohlfart P, Verheule S, Bohm M, Sadowski T, Schotten U. Cathepsin A mediates susceptibility to atrial 
tachyarrhythmia and impairment of atrial emptying function in Zucker diabetic fatty rats. Cardiovascular Research 2016;110:371-380.

12. Hatem SN, Sanders P. Epicardial adipose tissue and atrial fibrillation. Cardiovascular Research 2014;102:205-213.

13. Haissaguerre $M$, Lim KT, Jacquemet V, Rotter M, Dang L, Hocini M, Matsuo S, Knecht $\mathrm{S}$, Jais $\mathrm{P}$, Virag $\mathrm{N}$. Atrial fibrillatory cycle length: computer simulation and potential clinical importance. Europace 2007;9 Suppl 6:vi64-70.

14. Cheng Z, Deng H, Cheng K, Chen T, Gao P, Yu M, Fang Q. The amplitude of fibrillatory waves on leads aVF and V1 predicting the recurrence of persistent atrial fibrillation patients who underwent catheter ablation. Annals of Noninvasive Electrocardiology 2013;18:352-358.

15. Wijffels MC, Kirchhof CJ, Dorland R, Allessie MA. Atrial fibrillation begets atrial fibrillation. A study in awake chronically instrumented goats. Circulation 1995;92:1954-1968.

16. Allessie MA, de Groot NM, Houben RP, Schotten U, Boersma E, Smeets JL, Crijns HJ. Electropathological substrate of long-standing persistent atrial fibrillation in patients with structural heart disease: longitudinal dissociation. Circulation Arrhythmia and Electrophysiology 2010;3:606-615.

17. Lee S, Sahadevan J, Khrestian CM, Cakulev I, Markowitz A, Waldo AL. Simultaneous Bi-Atrial High Density (510 - 512 electrodes) Epicardial Mapping of Persistent and Long-Standing Persistent Atrial Fibrillation in Patients: New Insights into the Mechanism of Its Maintenance. Circulation 2015;132:2108-17.

18. Okin PM, Jern S, Devereux RB, Kjeldsen SE, Dahlof B. Effect of obesity on electrocardiographic left ventricular hypertrophy in hypertensive patients : the losartan intervention for endpoint (LIFE) reduction in hypertension study. Hypertension 2000;35:13-18.

19. Walters TE, Teh AW, Spence S, Morton JB, Kistler PM, Kalman JM. Absence of gender-based differences in the atrial and pulmonary vein substrate: a detailed electroanatomic mapping study. Journal of Cardiovascular Electrophysiology 2014;25:1065-1070.

20. Cochet $\mathrm{H}$, Mouries $\mathrm{A}$, Nivet $\mathrm{H}$, et al. Age, atrial fibrillation, and structural heart disease are the main determinants of left atrial fibrosis detected by delayedenhanced magnetic resonance imaging in a general cardiology population. Journal of Cardiovascular Electrophysiology 2015;26:484-492.

21. Chen YJ, Lee SH, Hsieh MH, Hsiao CJ, Yu WC, Chiou CW, Chen SA. Effects of 17 beta-estradiol on tachycardia-induced changes of atrial refractoriness and cisapride-induced ventricular arrhythmia. Journal of Cardiovascular Electrophysiology 1999;10:587-598. 


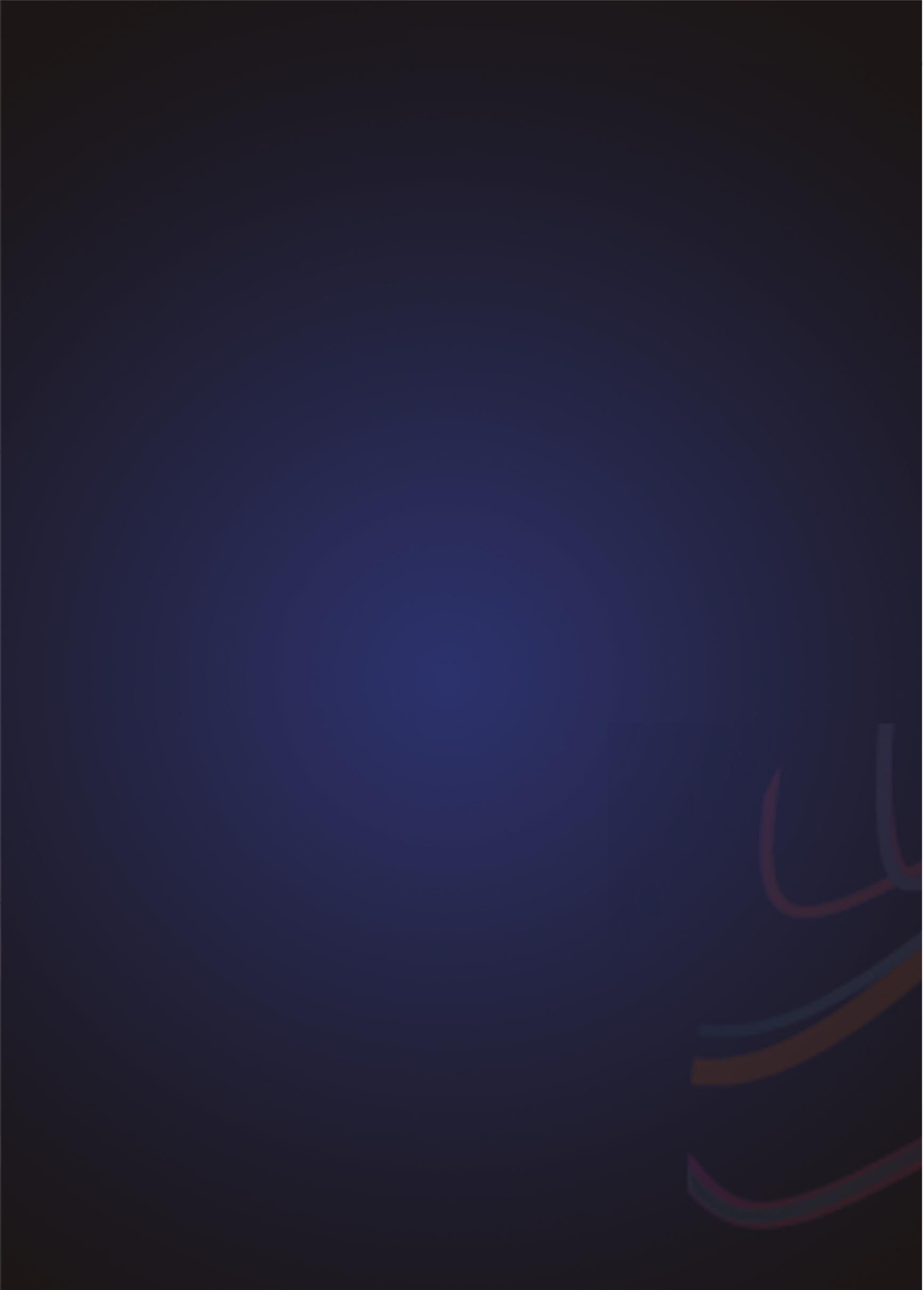




\section{Chapter 8}

Dominant frequency predicts sinus rhythm after electrical cardioversion for persistent atrial fibrillation in men but not in women

Theo Lankveld, Stef Zeemering, Harry J Crijns and Ulrich Schotten 
Chapter 8

\begin{abstract}
Aims: Women are usually underrepresented in studies evaluating rhythm control strategies in patients with atrial fibrillation (AF). Subsequently, the same holds true for studies looking at predictors for success of rhythm control. Whether clinical characteristics or parameters derived from the electrocardiogram (ECG) differ in their predictive value between women and men has not been explored yet. We studied the predictive power of recently identified non-invasive ECG predictors (dominant frequency of the f-waves) in men and women undergoing electrical cardioversion (ECV) of persistent AF.
\end{abstract}

Methods: We included female patients referred for ECV and individually matched them to male control patients referred for ECV. We determined the dominant frequency (DF) on all 12 leads of a standard 10 seconds ECG recorded on the day of ECV. Recurrences of AF within the first year after ECV were documented.

Results: We included 105 female and 105 male patients (age $68 \pm 9$ years) in the final analysis. The DF was significantly lower in patients without an AF recurrence in the combined groups in most leads. In males, the DF was significantly lower in all leads in patients without an AF recurrence. The best predicting lead was lead III with an AUC 0.75. The optimal cut-off point for sinus rhythm maintenance was a DF $<6.0 \mathrm{~Hz}$ with a $84 \%$ sensitivity and $67 \%$ specificity. There was no significant difference in DF between female patients with and without an AF recurrence (maximal predictor AUC 0.52 on lead V2).

Conclusion: The non-invasively measured f-wave dominant frequency is able to predict $A F$ recurrence after electrical cardioversion in male patients with persistent AF but not in a matched female cohort. More knowledge on pathophysiological differences between men and women is required to develop appropriate gender-specific prediction models. 


\section{Introduction}

Women are usually underrepresented in clinical trials evaluating rhythm control in patients with atrial fibrillation (AF). This is partly because AF is more prevalent in men than in women, ${ }^{1}$ but also because women more often receive rate than rhythm control therapy for $A F^{2,3}$ As a consequence, studies that aim to predict which patients are likely to benefit from certain rhythm control strategies include mainly male AF patients. We showed that the dominant frequency (DF) of the f-waves measured non-invasively on a standard 10 seconds 12 lead electrocardiogram (ECG) predicts sinus rhythm after electrical cardioversion (ECV) for persistent atrial fibrillation (AF). ${ }^{4}$ The DF is an easily obtainable parameter and therefore appealing to use in every day clinical practice. However, also in this study only $29 \%$ female patients were enrolled. Furthermore, there are reports that the DF is lower in female compared to male patients. ${ }^{5,6}$ Because of this underrepresentation and difference in $\mathrm{AF}$ cycle length between men and women, prediction models of rhythm outcome in general AF cohorts might be biased towards prediction of outcome in men. Therefore, we studied the predictive performance of the DF in women compared to a matched male control group in patients undergoing ECV for persistent AF.

\section{Methods}

\section{Population}

We included patients who underwent ECV for persistent AF at the Maastricht University Medical Centre, Maastricht, the Netherlands, that were prospectively included in a database between January 2008 and December 2014. The original database contained data of 348 men and 151 women. Female and male patients were individually matched. For each female patient a male patient was selected who fulfilled the following criteria: There was no difference in clinical co-morbidity (hypertension, diabetes mellitus, stroke, coronary artery disease and anti-arrhythmic drug use) and age matched with a range of \pm 1 year. If more than one male patient could be matched to a female patient, the male patient with the age most closely related to the female patient was chosen. If no suitable male candidate could be selected, the female patient was excluded from the analysis. The final database contained data of 105 male and 105 female patients. 


\begin{tabular}{|c|c|c|c|}
\hline & Male (105) & Female (105) & P-value \\
\hline Age & $68 \pm 9$ & $68 \pm 9$ & 0.834 \\
\hline HT & $61(58 \%)$ & $61(58 \%)$ & 1.000 \\
\hline DM & $5(5 \%)$ & $5(5 \%)$ & 1.000 \\
\hline Stroke & $2(2 \%)$ & $2(2 \%)$ & 1.000 \\
\hline CAD & 19 (18\%) & $19(18 \%)$ & 1.000 \\
\hline AAD & $18(17 \%)$ & $18(17 \%)$ & 1.000 \\
\hline Sotalol & $8(8 \%)$ & $9(9 \%)$ & \\
\hline Amiodarone & $9(9 \%)$ & $8(8 \%)$ & \\
\hline Flecainide & $1(1 \%)$ & $1(1 \%)$ & \\
\hline BMI & $28.3 \pm 3.8$ & $29.7 \pm 5.8$ & 0.078 \\
\hline Indexed LAV (ml/m2) & $44.7 \pm 12.7$ & $46.5 \pm 16.3$ & 0.401 \\
\hline AF episode (months) & $3[1-6]$ & $3[2-5]$ & 0.748 \\
\hline Recurrence 1 year & $68(65 \%)$ & 74 (70\%) & 0.376 \\
\hline
\end{tabular}

Table 1: Baseline characteristics. Anti-arrhythmic drug (AAD), atrial fibrillation (AF), body mass index (BMI), coronary artery disease (CAD), diabetes mellitus (DM), hypertension (HT), left atrial volume (LAV).

\section{Outcome}

The primary endpoint was recurrence of AF. Recurrence of AF was defined as failed ECV or documented AF on a 10 second ECG or on Holter monitoring during follow-up. $S R$ maintenance was defined as no documented AF recurrence within the first year after ECV. Routine control visits were conducted by the referring physician one month after ECV and thereafter as indicated necessary. The follow-up period consisted of one year. During each follow-up visit an ECG was recorded and symptoms were evaluated. If patients suffered from cardiac complaints, an ECG was recorded at the emergency department.

\section{Dominant frequency of fibrillation waves}

A 10 seconds 12 lead ECG was digitally recorded with a sampling frequency of $250 \mathrm{~Hz}$ at the day of ECV. The ECG was imported in custom-made Matlab (MATLAB 2013b, The MathWorks, Inc., Natick, Massachusetts, United States) software for the analysis. ECGs were filtered using a zero-phase band-pass filter between $1-100 \mathrm{~Hz}$ to remove baseline wandering and supress highfrequency noise. Powerline interference was removed using a $50 \mathrm{~Hz}$ notch 
filter. Ventricular signals were cancelled using a modified version of the adaptive singular value QRST cancellation. Before the analyses, an additional $3 \mathrm{~Hz}$ high-pass filter was applied to avoid interference caused by the (possible residual) T-wave. A frequency power spectrum was estimated using Welch's power spectral density estimate. The DF was defined as the frequency with the highest power between 3 and $12 \mathrm{~Hz}$ in this frequency spectrum.

\section{Statistical analysis}

Statistical analysis was performed using SPSS statistical software (IBM SPSS statistics version 21.0). Categorical variables are reported as number of patients and percentage. Continuous variables are presented as mean \pm standard deviation or as median with interquartile range depending on their distribution. Differences between continuous variables were compared using the Student's t-test in normally distributed data or the Mann-Whitney $U$ test otherwise. For the differences between the matched male and female population paired tests were used. A P-value of 0.05 was considered statistically significant.

\section{Results}

Out of the 151 female patients we were able to match 105 female patients with 105 male patients. There were no significant differences in DF between female patients included and excluded in this study (supplement table 1). The baseline characteristics of the patients included are shown in table 1. There were no differences in cardiovascular comorbidities, AF duration and indexed left atrial volume between the matched male and female patients (table 1). Within one year 68 (65\%) male patients and $74(70 \%)$ female patients experienced an AF recurrence ( $p=0.376)$.

In the total study population, the DF was higher in most leads in patients with a recurrence of AF within the first year after ECV (supplement table 2). The best performing lead was lead III with an AUC 0.62 (figure 1). There was a trend towards a higher DF in male than in female patients (supplement table 3 ). In the group of male patients, the DF was significantly higher in patients experiencing an AF recurrence (table 2). Best performing lead was lead III (AUC 0.75 ). The optimal cut-off point in lead III to predict SR maintenance was a DF < $6.0 \mathrm{~Hz}$ with a $84 \%$ sensitivity and a $67 \%$ specificity (log rank $p<0.001$ ). In female patients however, there was no significant difference in DF between patients with and without an AF recurrence (table 2), lead III had an AUC 0.47 (figure 1) and the maximal AUC was 0.52 in lead $\mathrm{V} 2$. 
Most clinical parameters did not predict AF recurrence in either men or women. Only AAD use (AUC 0.60) and high age (AUC 0.68) predicted SR maintenance in male but not in female patients (table 3 ).
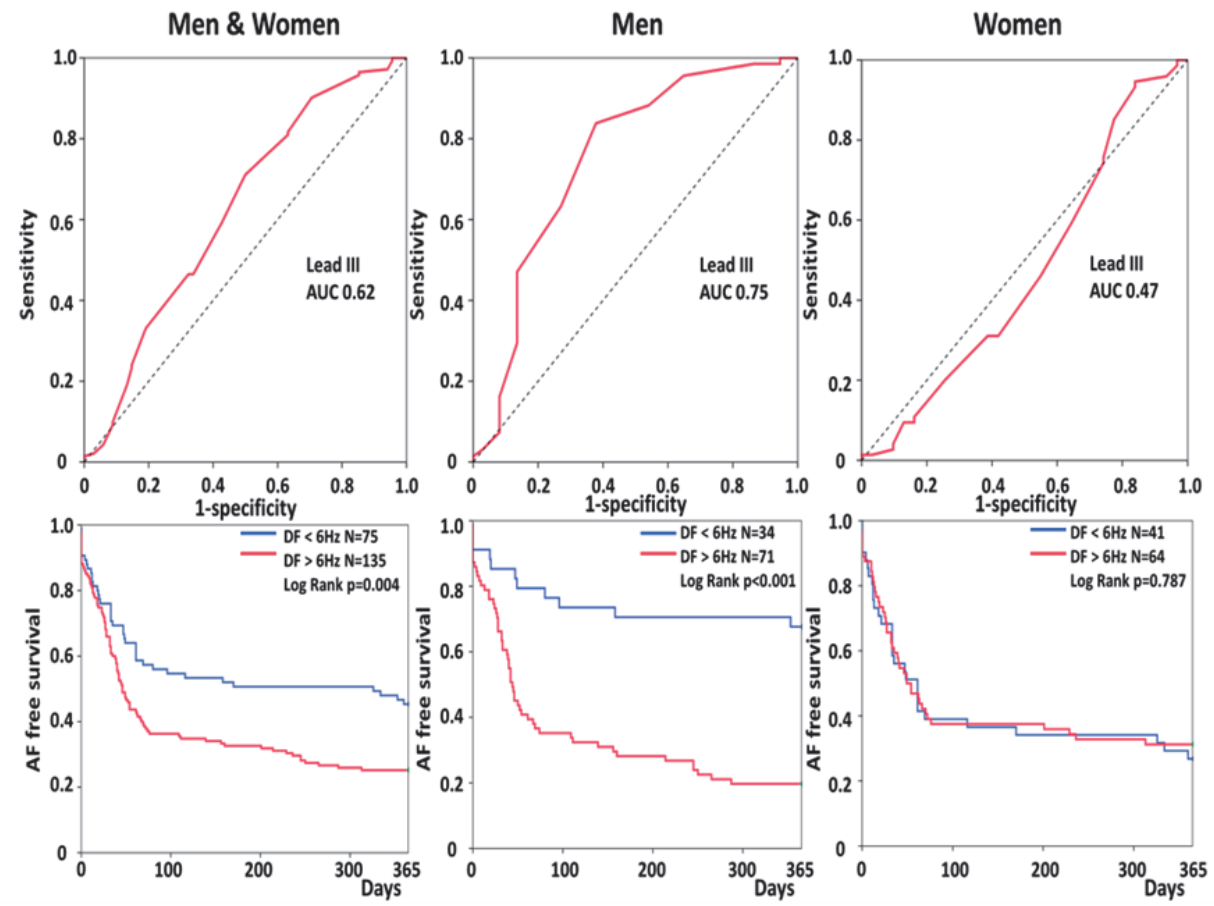

Figure 1: Figure 1 shows the receiver operator characteristic (ROC) curves for the best performing lead (lead III) in the combined population (left), the male (middle) and female studygroup (right). The bottom half shows the AF free survival curves for the optimal cut-off point determined from the ROC curve. This figure shows the ability of the dominant frequency (DF) to predict sinus rhythm maintenance after 1 year follow-up in male patients but not in female patients.

\section{Discussion}

Similarly to previous investigations, the present study showed that a lower DF indicates a more favourable rhythm outcome. ${ }^{4,7}$ However, this is the first study that showed that a lower DF predicts SR maintenance after ECV in male patients with persistent AF but not in a matched female cohort. No previous studies investigated gender-related difference in outcome prediction in patients undergoing ECV for AF. There are studies however, on the predictive value of clinical parameters for the prediction of catheter ablation outcome stratified by gender. ${ }^{8,9}$ Age, AF duration, number of failed anti-arrhythmic drugs and indexed left atrial diameter predicted tachyarrhythmia recurrence in 
men but not in women. ${ }^{8}$ Also BNP, a known predictor for success of rhythm control, predicts recurrence of tachyarrhythmia's after catheter ablation in men but not in women. ${ }^{9}$ It is difficult to explain these differences because only little research has been conducted on gender-related pathophysiological AF mechanisms. Interestingly, a study with rabbits showed faster spontaneous activity in the pulmonary veins of male rabbits with a higher incidence of burst firing. ${ }^{10}$ A human study from Walters and colleagues showed no significant differences in atrial electrophysiological substrate between men and women. ${ }^{11}$ In contrast, other studies showed a higher incidence of non-pulmonary vein triggers in women. $8,12,13$ This could be a result of later referral and therefore more advanced electrophysiological remodelling but could also suggest that in women the quantitative contribution of triggers to AF is more pronounced than in men. AF triggers cannot be identified by the DF and therefore do not strengthen this parameter as a predictor for recurrences.

\begin{tabular}{|c|c|c|c|c|c|c|}
\hline lead & $\begin{array}{c}\text { Female } \\
\text { AF } \\
\text { recurrence } \\
(N=74)\end{array}$ & $\begin{array}{c}\text { Female } \\
\text { No AF } \\
\text { recurrence } \\
(\mathbf{N}=31)\end{array}$ & $\begin{array}{c}p- \\
\text { value }\end{array}$ & $\begin{array}{c}\text { Male } \\
\text { AF } \\
\text { recurrence } \\
(N=68)\end{array}$ & $\begin{array}{c}\text { Male } \\
\text { No AF } \\
\text { recurrence } \\
(\mathbf{N}=37)\end{array}$ & $\begin{array}{c}p- \\
\text { value }\end{array}$ \\
\hline$I$ & $6.32 \pm 0.78$ & $6.36 \pm 0.91$ & 0.846 & $6.73 \pm 0.78$ & $6.17 \pm 0.98$ & 0.002 \\
\hline II & $6.17 \pm 0.69$ & $6.19 \pm 0.95$ & 0.900 & $6.63 \pm 0.73$ & $6.13 \pm 0.95$ & 0.003 \\
\hline III & $6.20 \pm 0.71$ & $6.22 \pm 0.89$ & 0.875 & $6.70 \pm 0.74$ & $5.96 \pm 0.90$ & $<0.001$ \\
\hline aVR & $6.24 \pm 0.71$ & $6.36 \pm 0.95$ & 0.523 & $6.58 \pm 0.71$ & $6.10 \pm 0.99$ & 0.013 \\
\hline $\mathrm{aVL}$ & $6.30 \pm 0.75$ & $6.22 \pm 0.87$ & 0.638 & $6.63 \pm 0.74$ & $6.08 \pm 0.96$ & 0.002 \\
\hline aVF & $6.19 \pm 0.70$ & $6.25 \pm 0.96$ & 0.704 & $6.66 \pm 0.73$ & $6.14 \pm 1.03$ & 0.008 \\
\hline V1 & $6.42 \pm 0.74$ & $6.41 \pm 0.91$ & 0.956 & $6.78 \pm 0.73$ & $6.24 \pm 1.08$ & 0.008 \\
\hline V2 & $6.39 \pm 0.80$ & $6.36 \pm 0.91$ & 0.894 & $6.70 \pm 0.72$ & $6.35 \pm 1.12$ & 0.101 \\
\hline V3 & $6.36 \pm 0.72$ & $6.37 \pm 0.88$ & 0.934 & $6.58 \pm 0.74$ & $6.15 \pm 0.88$ & 0.010 \\
\hline V4 & $6.28 \pm 0.72$ & $6.36 \pm 0.93$ & 0.677 & $6.62 \pm 0.75$ & $6.12 \pm 0.85$ & 0.002 \\
\hline V5 & $6.24 \pm 0.75$ & $6.25 \pm 0.89$ & 0.918 & $6.58 \pm 0.78$ & $6.16 \pm 0.93$ & 0.015 \\
\hline V6 & $6.32 \pm 0.79$ & $6.28 \pm 0.91$ & 0.802 & $6.63 \pm 0.85$ & $6.20 \pm 1.05$ & 0.024 \\
\hline
\end{tabular}

Table 2: Dominant frequency per lead divided per gender. Atrial fibrillation (AF). 


\section{Dominant frequency and gender}

We observed a trend towards a lower DF in women. Some studies suggested that women have a significantly lower DF of the f-waves in the ECG than men."

${ }^{6}$ Despite this more favourable atrial rate, a rhythm control strategy fails at least as often in women as it does in men. A meta-analysis showed that female patients undergoing catheter ablation for AF have a higher risk of AF recurrence after ablation than men. ${ }^{14}$ Women more frequently had persistent $\mathrm{AF}$ and were referred later in the course of the disease both limiting ablation success. ${ }^{14}$ However, a recently published registry showed less favourable ablation outcome even in female patients with paroxysmal AF. ${ }^{15}$ The present study showed no significant difference in recurrence rates between men and women. A possible explanation for the lower DF without the favourable rhythm outcome in female patients could be the higher percentage of atrial fibrosis in women with cardiovascular disease. ${ }^{16}$ Atrial fibrosis is an independent predictor of arrhythmia recurrence after catheter ablation, ${ }^{17}$ and also is associated with a lower DF. ${ }^{18,19}$ The higher oestrogen levels in women could be another explanation for their lower DF. Dogs that received $17 \beta$ estradiol had prolonged atrial effective refractory periods during atrial tachycardia. ${ }^{20}$ In a small study with postmenopausal women, $17 \beta$-estradiol also prolonged the right atrial effective refractory period. ${ }^{21}$ Furthermore, during the luteal phase women experience more and longer episodes of supraventricular arrhythmias than during the follicular phase of the menstrual cycle. ${ }^{22}$ However, most women in the present study are postmenopausal and therefore hormonal influences are likely less important in this study.

\section{Conclusions}

This study shows that the DF of F-waves on a standard 12 lead ECG can be used to predict recurrences of AF after electrical cardioversion in men but not in women. The lack of predictive power on rhythm outcome in women does not only seem to be confined to the ECG but appears to be a problem also when clinical parameters are used to predict outcome. Therefore future research is needed on pathophysiological differences between men and women that can explain these differences and may help to overcome the limited predictive performance of ECG and clinical parameters in women. 


\section{Limitations}

Rhythm follow-up was not standardised, nor daily rhythm follow-up was obtained. Therefore, especially self-limiting paroxysmal episodes might have been missed. In this population however, we expect most patients to experience recurrences of persistent rather than paroxysmal AF. Female AF patients experience more AF-related symptoms and have a lower quality of life than men which may have resulted into more frequent rhythm follow-up and therefore earlier detection of an AF recurrence. ${ }^{3,23}$

\begin{tabular}{|c|c|c|c|c|c|c|}
\hline & $\begin{array}{c}\text { Female } \\
\text { AF recurrence } \\
(\mathrm{N}=74)\end{array}$ & $\begin{array}{l}\text { Female } \\
\text { No AF } \\
\text { recurrence } \\
(\mathrm{N}=\mathbf{3 1})\end{array}$ & $\begin{array}{c}\mathrm{p}- \\
\text { value }\end{array}$ & $\begin{array}{c}\text { Male } \\
\text { AF } \\
\text { recurrence } \\
(\mathrm{N}=68)\end{array}$ & $\begin{array}{c}\text { Male } \\
\text { No AF } \\
\text { recurrence } \\
(\mathrm{N}=37)\end{array}$ & $\begin{array}{c}\mathrm{p}- \\
\text { value }\end{array}$ \\
\hline Age & $69 \pm 8$ & $65 \pm 12$ & 0.093 & $66 \pm 9$ & $71 \pm 10$ & 0.024 \\
\hline HT & 45 (61\%) & $16(52 \%)$ & 0.384 & $39(57 \%)$ & $22(60 \%)$ & 0.834 \\
\hline DM & $5(7 \%)$ & $0(0 \%)$ & 0.138 & $4(6 \%)$ & $1(3 \%)$ & 0.465 \\
\hline Stroke & $1(1 \%)$ & $1(3 \%)$ & 0.522 & $2(3 \%)$ & $0(0 \%)$ & 0.292 \\
\hline CAD & $16(22 \%)$ & $3(10 \%)$ & 0.147 & $12(18 \%)$ & $7(19 \%)$ & 0.872 \\
\hline AAD & $12(16 \%)$ & 6 (19\%) & 0.697 & $7(10 \%)$ & $11(30 \%)$ & 0.012 \\
\hline BMI & $29.6 \pm 5.9$ & $29.9 \pm 5.6$ & 0.773 & $28.4 \pm 4.0$ & $28.3 \pm 3.7$ & 0.926 \\
\hline $\begin{array}{l}\text { Indexed } \\
\text { LAV } \\
(\mathrm{ml} / \mathrm{m} 2)\end{array}$ & $45 \pm 16$ & $48 \pm 16$ & 0.404 & $46.6 \pm 13.4$ & $45.5 \pm 15.0$ & 0.739 \\
\hline $\begin{array}{l}\text { AF } \\
\text { episode } \\
\text { (months) }\end{array}$ & $2.5[2-5.25]$ & $3[1.75-5.25]$ & 0.586 & $3[2-6.75]$ & $2[1-5.50]$ & 0.086 \\
\hline
\end{tabular}

Table 3: Clinical characteristics predicting atrial fibrillation (AF) recurrence. Anti-arrhythmic drug $(A A D)$, atrial fibrillation $(A F)$, body mass index (BMI), coronary artery disease (CAD), diabetes mellitus (DM), hypertension (HT), left atrial volume (LAV). 


\section{Chapter 8}

\section{References}

1. Chugh SS, Havmoeller R, Narayanan K, et al. Worldwide epidemiology of atrial fibrillation: a Global Burden of Disease 2010 Study. Circulation Feb 25 2014;129:837-847.

2. Dagres N, Nieuwlaat R, Vardas PE, Andresen D, Levy S, Cobbe S, Kremastinos DT, Breithardt G, Cokkinos DV, Crijns HJ. Gender-related differences in presentation, treatment, and outcome of patients with atrial fibrillation in Europe: a report from the Euro Heart Survey on Atrial Fibrillation. Journal of the American College of Cardiology Feb 6 2007;49:572-577.

3. Lip GY, Laroche C, Boriani G, et al. Sex-related differences in presentation, treatment, and outcome of patients with atrial fibrillation in Europe: a report from the Euro Observational Research Programme Pilot survey on Atrial Fibrillation. Europace : European pacing, arrhythmias, and cardiac electrophysiology : journal of the working groups on cardiac pacing, arrhythmias, and cardiac cellular electrophysiology of the European Society of Cardiology Jan 2015;17:24-31.

4. Lankveld T, de Vos CB, Limantoro I, Zeemering S, Dudink E, Crijns HJ, Schotten U. Systematic Analysis of ECG Predictors for Sinus Rhythm Maintenance Following Electrical Cardioversion for Persistent Atrial Fibrillation. Heart rhythm : the official journal of the Heart Rhythm Society Jan 82016.

5. Bollmann A, Tveit A, Husser D, Stridh M, Sornmo L, Smith P, Olsson SB. Fibrillatory rate response to candesartan in persistent atrial fibrillation. Europace : European pacing, arrhythmias, and cardiac electrophysiology : journal of the working groups on cardiac pacing, arrhythmias, and cardiac cellular electrophysiology of the European Society of Cardiology Oct 2008;10:1138-1144.

6. Choudhary MB, Holmqvist F, Carlson J, Nilsson HJ, Roijer A, Platonov PG. Low atrial fibrillatory rate is associated with spontaneous conversion of recentonset atrial fibrillation. Europace : European pacing, arrhythmias, and cardiac electrophysiology : journal of the working groups on cardiac pacing, arrhythmias, and cardiac cellular electrophysiology of the European Society of Cardiology Oct 2013;15:1445-1452.

7. Holmqvist F, Stridh M, Waktare JE, Sornmo L, Olsson SB, Meurling CJ. Atrial fibrillatory rate and sinus rhythm maintenance in patients undergoing cardioversion of persistent atrial fibrillation. European heart journal Sep 2006;27:2201-2207.

8. Takigawa M, Kuwahara T, Takahashi A, et al. Differences in catheter ablation of paroxysmal atrial fibrillation between males and females. International journal of cardiology Oct 3 2013;168:1984-1991.

9. Mohanty S, Mohanty $P$, Di Biase L, Rong B, Burkhardt D, Gallinghouse JG, Horton R, Sanchez JE, Bailey S, Zagrodzky J, Natale A. Baseline B-type natriuretic peptide: a gender-specific predictor of procedure-outcome in atrial 
fibrillation patients undergoing catheter ablation. Journal of cardiovascular electrophysiology Aug 2011;22:858-865.

10. Tsai WC, Chen YC, Lin YK, Chen SA, Chen YJ. Sex differences in the electrophysiological characteristics of pulmonary veins and left atrium and their clinical implication in atrial fibrillation. Circulation Arrhythmia and electrophysiology Aug 2011;4:550-559.

11. Walters TE, Teh AW, Spence S, Morton JB, Kistler PM, Kalman JM. Absence of gender-based differences in the atrial and pulmonary vein substrate: a detailed electroanatomic mapping study. Journal of cardiovascular electrophysiology Oct 2014;25:1065-1070.

12. Lee $\mathrm{SH}$, Tai CT, Hsieh MH, Tsao HM, Lin YJ, Chang SL, Huang JL, Lee KT, Chen YJ, Cheng JJ, Chen SA. Predictors of non-pulmonary vein ectopic beats initiating paroxysmal atrial fibrillation: implication for catheter ablation. Journal of the American College of Cardiology Sep 20 2005;46:1054-1059.

13. Patel D, Mohanty P, Di Biase $L$, et al. Outcomes and complications of catheter ablation for atrial fibrillation in females. Heart rhythm : the official journal of the Heart Rhythm Society 2010;7:167-172.

14. Vallakati A, Reddy M, Sharma A, et al. Impact of gender on outcomes after atrial fibrillation ablation. International journal of cardiology 2015;187:12-16.

15. Zylla MM, Brachmann J, Lewalter T, et al. Sex-related outcome of atrial fibrillation ablation: Insights from the German Ablation Registry. Heart rhythm : the official journal of the Heart Rhythm Society Jun 82016.

16. Cochet $\mathrm{H}$, Mouries $\mathrm{A}$, Nivet $\mathrm{H}$, et al. Age, atrial fibrillation, and structural heart disease are the main determinants of left atrial fibrosis detected by delayedenhanced magnetic resonance imaging in a general cardiology population. Journal of cardiovascular electrophysiology May 2015;26:484-492.

17. Marrouche NF, Wilber D, Hindricks G, et al. Association of atrial tissue fibrosis identified by delayed enhancement $\mathrm{MRI}$ and atrial fibrillation catheter ablation: the DECAAF study. Jama Feb 5 2014;311:498-506.

18. Koduri H, Ng J, Cokic I, Aistrup GL, Gordon D, Wasserstrom JA, Kadish AH, Lee R, Passman R, Knight BP, Goldberger JJ, Arora R. Contribution of fibrosis and the autonomic nervous system to atrial fibrillation electrograms in heart failure. Circulation Arrhythmia and electrophysiology Aug 1 2012;5:640-649.

19. Swartz MF, Fink GW, Lutz CJ, Taffet SM, Berenfeld O, Vikstrom KL, Kasprowicz K, Bhatta L, Puskas F, Kalifa J, Jalife J. Left versus right atrial difference in dominant frequency, $\mathrm{K}(+)$ channel transcripts, and fibrosis in patients developing atrial fibrillation after cardiac surgery. Heart rhythm : the official journal of the Heart Rhythm Society Oct 2009;6:1415-1422.

20. Chen YJ, Lee SH, Hsieh MH, Hsiao CJ, Yu WC, Chiou CW, Chen SA. Effects of 17beta-estradiol on tachycardia-induced changes of atrial refractoriness and cisapride-induced ventricular arrhythmia. Journal of cardiovascular electrophysiology Apr 1999;10:587-598.

21. Rosano GM, Leonardo F, Dicandia C, Sheiban I, Pagnotta P, Pappone C, Chierchia SL. Acute electrophysiologic effect of estradiol 17beta in 
Chapter 8

menopausal women. The American journal of cardiology Dec 15 2000;86:1385-1387, A1385-1386.

22. Rosano GM, Leonardo F, Sarrel PM, Beale CM, De Luca F, Collins P. Cyclical variation in paroxysmal supraventricular tachycardia in women. Lancet Mar 23 1996;347:786-788.

23. Rienstra M, Van Veldhuisen DJ, Hagens VE, Ranchor AV, Veeger NJ, Crijns HJ, Van Gelder IC. Gender-related differences in rhythm control treatment in persistent atrial fibrillation: data of the Rate Control Versus Electrical Cardioversion (RACE) study. Journal of the American College of Cardiology Oct 4 2005;46:1298-1306. 


\section{Supplements}

\begin{tabular}{cccc}
\hline lead & $\begin{array}{c}\text { Included } \\
(\mathbf{N}=\mathbf{1 0 5})\end{array}$ & $\begin{array}{c}\text { Excluded } \\
(\mathbf{N}=\mathbf{4 6})\end{array}$ & p-value \\
\hline I & $6.33 \pm 0.82$ & $6.16 \pm 0.86$ & 0.236 \\
II & $6.17 \pm 0.77$ & $6.23 \pm 0.94$ & 0.720 \\
III & $6.20 \pm 0.76$ & $6.25 \pm 0.79$ & 0.721 \\
aVR & $6.28 \pm 0.78$ & $6.18 \pm 0.94$ & 0.520 \\
aVL & $6.28 \pm 0.79$ & $6.35 \pm 0.87$ & 0.628 \\
aVF & $6.21 \pm 0.78$ & $6.29 \pm 0.80$ & 0.534 \\
V1 & $6.42 \pm 0.79$ & $6.45 \pm 0.96$ & 0.809 \\
V2 & $6.38 \pm 0.83$ & $6.41 \pm 0.98$ & 0.841 \\
V3 & $6.36 \pm 0.77$ & $6.25 \pm 0.84$ & 0.433 \\
V4 & $6.31 \pm 0.79$ & $6.16 \pm 0.79$ & 0.285 \\
V5 & $6.24 \pm 0.79$ & $6.20 \pm 0.85$ & 0.800 \\
V6 & $6.31 \pm 0.82$ & $6.21 \pm 0.85$ & 0.504 \\
\hline
\end{tabular}

Table 1: Dominant frequency included and excluded female patients.

\begin{tabular}{cccc}
\hline lead & $\begin{array}{c}\text { AF recurrence } \\
(\mathbf{N}=\mathbf{1 0 5})\end{array}$ & $\begin{array}{c}\text { No AF recurrence } \\
(\mathbf{N}=\mathbf{4 6})\end{array}$ & p-value \\
\hline I & $6.52 \pm 0.80$ & $6.25 \pm 0.95$ & 0.038 \\
II & $6.39 \pm 0.74$ & $6.16 \pm 0.94$ & 0.078 \\
III & $6.44 \pm 0.76$ & $6.08 \pm 0.90$ & 0.003 \\
aVR & $6.40 \pm 0.72$ & $6.22 \pm 0.97$ & 0.177 \\
aVL & $6.46 \pm 0.76$ & $6.15 \pm 0.91$ & 0.010 \\
aVF & $6.41 \pm 0.75$ & $6.19 \pm 0.99$ & 0.102 \\
V1 & $6.59 \pm 0.76$ & $6.32 \pm 1.00$ & 0.048 \\
V2 & $6.54 \pm 0.78$ & $6.36 \pm 1.02$ & 0.211 \\
V3 & $6.46 \pm 0.74$ & $6.25 \pm 0.88$ & 0.069 \\
V4 & $6.44 \pm 0.75$ & $6.23 \pm 0.87$ & 0.065 \\
V5 & $6.40 \pm 0.79$ & $6.20 \pm 0.90$ & 0.104 \\
V6 & $6.47 \pm 0.83$ & $6.24 \pm 0.98$ & 0.072 \\
\hline
\end{tabular}

Table 2: Dominant frequency in patients with and without an atrial fibrillation (AF) recurrence. 
Chapter 8

\begin{tabular}{cccc}
\hline lead & $\begin{array}{c}\text { Male } \\
(\mathbf{N}=\mathbf{1 0 5})\end{array}$ & $\begin{array}{c}\text { Female } \\
(\mathbf{N}=\mathbf{1 0 5})\end{array}$ & p-value \\
\hline I & $6.53 \pm 0.89$ & $6.32 \pm 0.83$ & 0.054 \\
II & $6.46 \pm 0.84$ & $6.17 \pm 0.77$ & 0.007 \\
III & $6.45 \pm 0.87$ & $6.21 \pm 0.75$ & 0.027 \\
aVR & $6.42 \pm 0.85$ & $6.28 \pm 0.78$ & 0.152 \\
aVL & $6.43 \pm 0.86$ & $6.28 \pm 0.79$ & 0.163 \\
aVF & $6.48 \pm 0.88$ & $6.20 \pm 0.78$ & 0.010 \\
V1 & $6.61 \pm 0.90$ & $6.42 \pm 0.79$ & 0.058 \\
V2 & $6.58 \pm 0.89$ & $6.39 \pm 0.82$ & 0.083 \\
V3 & $6.44 \pm 0.82$ & $6.36 \pm 0.77$ & 0.397 \\
V4 & $6.45 \pm 0.82$ & $6.31 \pm 0.78$ & 0.206 \\
V5 & $6.44 \pm 0.86$ & $6.24 \pm 0.79$ & 0.071 \\
V6 & $6.48 \pm 0.94$ & $6.31 \pm 0.82$ & 0.106 \\
\hline
\end{tabular}

Table 3: Dominant frequency in male and female patients. 



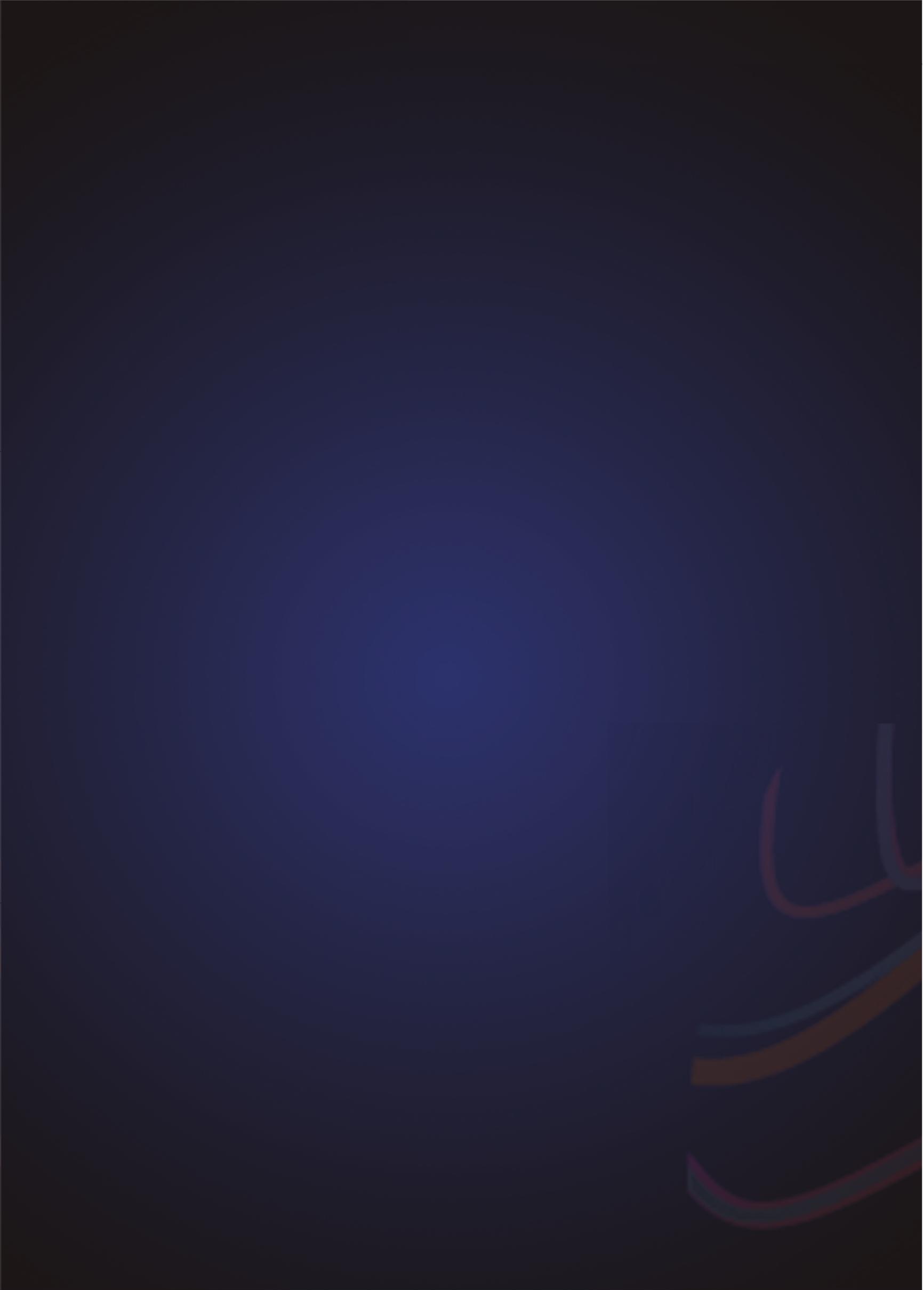




\section{Chapter 9}

General discussion 
Chapter 9

\section{Chapter 9 General Discussion}

The objectives of this thesis were:

1. To identify easily obtainable ECG-derived parameters that help to identify patients at risk for atrial fibrillation.

2. To identify ECG-derived parameters that predict success of a rhythm control strategy.

3. To study the association of candidate ECG parameters for rhythm outcome with age, gender and comorbidities.

As a first step we identified ECG-parameters in sinus rhythm ECGs that distinguish between patients with and without atrial fibrillation (AF) in the history (Part I). In the second part of this thesis we identified several promising easily obtainable ECG-derived parameters that can predict rhythm outcome in patients undergoing pharmacological cardioversion of AF or recurrences of AF following electrical cardioversion or catheter ablation (Part II). Part III of this thesis focuses on the influence of clinical parameters on these complexity and frequency parameters. The last part of this discussion places the findings of this thesis in clinical perspective and explains future research directions.

\section{Part I: The electrocardiogram during sinus rhythm}

For the initiation and perpetuation of AF a trigger (for example a supraventricular extra stimulus) and a substrate is required. We studied ECG parameters that reflect the atrial conduction and therefore possibly also conduction abnormalities representing the underlying atrial substrate. An association between the duration of the P-wave and left atrial enlargement has been described decades ago. ${ }^{1,2}$ Since this time, software used on scanned tracings improved reproducibility of the measurement of the P-wave duration. ${ }^{3}$ One could argue that digitally recorded and exported ECGs can improve the accuracy and reproducibility of the quantitative description of P-wave properties. Still, the exact determination of the end of the P-wave remains a challenging task. The most commonly used ECG-recording devices use heavy filtering for noise reduction but heavy filtering abolishes relevant details within the P-wave. In chapter $\mathbf{2}$ of this thesis we used signal averaging of the P-wave to reduce the noise levels and identify robust peaks within the $\mathrm{P}$-wave as reported before. ${ }^{4}$ We showed that the mean number of peaks within the Pwaves located over the body surface can distinguish between patients with a history of AF and individuals without AF. More importantly, the discriminative power of the peaks within the P-wave between patients with and without AF is spatially not homogenously distributed. Leads located on the high left 
posterior side of the body surface seem of most value. With ECG-imaging we showed that left atrial activation is best represented in these leads (figure 4 of chapter 2). A recent study using models of the human atria to simulate atrial activation on the body surface also showed that important information is located on the posterior left torso. ${ }^{5}$ The higher number of peaks in these leads could represent local conduction disturbances due to atrial fibrosis or infiltration of adipose tissue. ${ }^{6}$ The left atrial posterior wall is a known location predisposed to fibrosis and epicardial fat. ${ }^{7-9}$

\section{Part II: The electrocardiogram predicting a rhythm control strategy}

High density epicaridal mapping during atrial fibrillation provided insights in the pathophysiology of the arrhythmia. ${ }^{10-13}$ These studies have demonstrated an increase in the degree of conduction block, more and smaller fibrillatory waves, increasing numbers of breakthroughs and endo-epicardial dissociation in more persistent forms of AF. Direct contact mapping can provide detailed information about the electrophysiological complexity of an individual patient but can only be done in patients undergoing open chest surgery. A 10 seconds 12 lead ECG is available in every patient with AF and therefore an appealing diagnostic tool to use. Although a standard 12 lead ECG provides more global information, there are some regional differences that can be observed. In chapter 6 we confirmed the finding that the global atrial activation rate measured in the right or left atrial appendage can be measured reliably on the standard 12 lead ECG. We showed that the activation rate of the right atrial appendage is best captured by lead V1 and the activation rate of the left atrial appendage in leads I, aVL and V6.

The decision for a rate or rhythm control strategy and the type of rhythm control strategy is usually based on the patient's and physician's preference combined with clinical predictors for success of a certain therapy. Instead of selecting a certain treatment strategy based on preferences, identifying the appropriate treatment based on electrophysiological properties and comorbidities of a patient seems more logical. An important parameter predicting success of rhythm outcome is a short duration of the current AF

episode. ${ }^{14-16}$ However, the exact duration of the AF episode is often difficult to determine due to vague symptoms or even asymptomatic episodes. Left atrial dilatation could also predict failure of rhythm control but study results are inconsistent. ${ }^{17-19}$ Therefore, there is a need for easily obtainable robust predictors of rhythm outcome.

As discussed before, the ECG is able to identify certain electrophysiological characteristics and can potentially identify patients with a low and highly 
complex atrial substrate. As a next step towards an individualised therapy strategy based on non-invasive ECG-derived complexity parameters we evaluated these ECG parameters as predictors of successful treatment. In this thesis we examined a wide variety of patient cohorts to address this question.

Classically, AF patients are divided in patients with paroxysmal, persistent and long-standing persistent AF mainly based on the duration of the AF episodes. In chapter $\mathbf{3}$ of this thesis we showed that most studies on ECG-derived complexity parameters include one group of AF patients and a small selection of complexity parameters. This thesis covers the full range of patients from paroxysmal to long-standing persistent forms of AF. In chapter $\mathbf{4}$ we showed that a low DF identifies patients with short-lasting paroxysmal AF likely to respond to intravenous flecainide. Chapter $\mathbf{5}$ showed that a lower DF predicts successful rhythm control in a large cohort of patients with persistent AF undergoing electrical cardioversion for AF followed for one year. We were not able to predict early recurrences of AF. A possible explanation could be the high occurrence and influence of triggers rather than the electrophysiological substrate within the first days to weeks after cardioversion. In chapter 6 we demonstrated that in patients with long-standing persistent AF a high F-wave amplitude (FWA) predicted acute and long-term success of stepwise catheter ablation in two separate cohorts. Previous studies also showed the predictive value of a lower DF on success of catheter ablation. ${ }^{20,21}$ In our study we found no predictive value of the DF for long-term success in the training cohort. There was however limited predictive value of a lower DF on rhythm outcome in the validation cohort. A possible explanation for the limited predictive value of the DF in patients with long-standing persistent AF could be the little change in DF after 1-2 years as explained later in this discussion. ${ }^{22}$

In all these studies we examined a wide variety of frequency and complexity parameters. Interestingly, easily understandable parameters like the DF and FWA rather than complex mathematical parameters such as spectral of signal entropy seem to harbour the most clinically relevant information. A possible explanation for this might be that these parameters are less likely to be affected by signal quality or filter settings used in the ECG hardware. The ECGs used in these studies were recorded as part of clinical practice but not for detailed signal analysis. This means that the parameters identified in this study could easily be implemented in everyday clinical practice.

\section{Part III: ECG derived parameters in clinical context}

In chapter $\mathbf{7}$ we showed that the clinical characteristics of the patients have a substantial influence on the ECG-derived frequency and complexity 
parameters. For instance, high age and comorbidities are associated with a low DF. This may have consequences for models predicting rhythm outcome. While a lower DF identifies patients likely to respond to rhythm control therapy (chapter 4 and 5), a higher number of comorbidities also lowers the DF partially counteracting the effect of a low DF on outcome as such. Furthermore, a previous study showed that a lower DF is independently associated with a higher mortality rate in patients with chronic heart failure. ${ }^{23}$ For FWA the influence of clinical characteristics and amplitude in outcome prediction of a rhythm control strategy seems more logical. An increasing number of comorbidities lowers the FWA, a lower FWA also means a lower chance of success of catheter ablation (chapter 6). The fact that the combination of clinical and ECG predictors improves outcome prediction models over only clinical or only ECG prediction models demonstrates that clinical and ECG parameters contain complemental information (chapter 4-6).

We hypothesise that with increasing duration of the AF episodes the DF increases first and thereafter reaches a plateau phase ${ }^{22,24}$ or even declines at a later stage (figure 1 ). This hypothesis is corroborated by the notion that the DF is lower in paroxysmal than in persistent AF patients (chapter 4 and 5) and unpublished data on 39 patients with persistent AF followed for a 8 years without significant change in DF (figure 2). The AF cycle length (AFCL) (inversely correlated to the DF) is composed of the atrial effective refractory period (AERP) and the excitable gap. During the initial days of AF the AERP and thus the AFCL shorten. ${ }^{25}$ The progressive shortening of the AFCL over the next months ${ }^{24}$ could be the result of a shortening of the excitable gap potentially explained by the increasing number of atrial fibrillation waves ${ }^{10}$ and therefore the increasing chance of reactivation of the atrial tissue after the previous activation. After this period the AFCL may level off in clinically stable patients with persistent AF (figure 2). ${ }^{22}$ However, an increasing degree of atrial fibrosis may eventually results in a slight increase in the AFCL. ${ }^{26,27}$ The latter might explain the lower DF with increasing age and comorbidities (chapter 7). The FWA shows a more uniform change over time. During the increasing persistence of AF the FWA decreases (figure 2) possibly due to the accumulation of atrial fibrosis resulting in higher complexity of AF. 

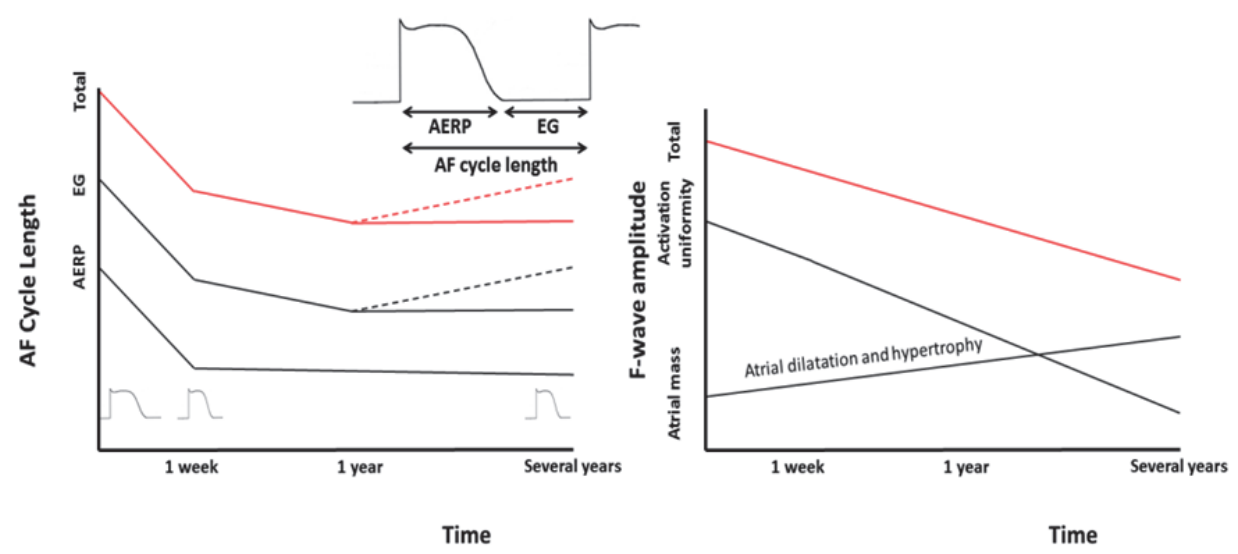

Figure 1: This figure shows the hypothetical evolution of the atrial fibrillation (AF) cycle length (inversely correlated with the dominant frequency) and the F-wave amplitude on the AF ECG with increasing episode duration. During the first days of $A F$ the atrial effective refractory period (AERP) shortens. Therefore, the AF cycle length shortens in the first days. During the first year the excitable gap (EG) continues to shorten, resulting in the progressive shortening of the AF cycle length. After the first year the AF cycle length remains relatively stable. However, the EG might increase due to atrial fibrosis resulting in an increase in AF cycle length. The F-wave amplitude seems to decrease with an increase in AF duration. This could be explained by the more disorganised atrial activation. The progressive atrial dilatation and hypertrophy during AF, which could increase the F-wave amplitude due to a larger atrial mass, seems not to outweigh this effect .

Not only comorbidities influence ECG-parameters but also gender. In chapter 7 we confirmed that women have a lower DF than men. ${ }^{28,} 29$ The exact mechanism for the lower DF in women has yet to be elucidated. Possibly hormonal influences ${ }^{30}$ or the higher amount of fibrosis in female AF patients ${ }^{7}$ play a role. We not only observed gender differences in the absolute values of DF during AF but gender also affected the predictive value of clinical and ECG parameters. In chapter $\mathbf{5}$ we showed that the DF was the best electrocardiographic parameter for SR maintenance after ECV. However, in chapter $\mathbf{8}$ we demonstrated that the DF predicts outcome in men but not in women. The explanation for this discrepancy in outcome prediction is not known so far. Interestingly, also other predictors for rhythm control success seem to predict rhythm outcome more accurately in men. ${ }^{31}$ This certainly stresses the need for more research regarding differences in the AF pathophysiology between men and women. 

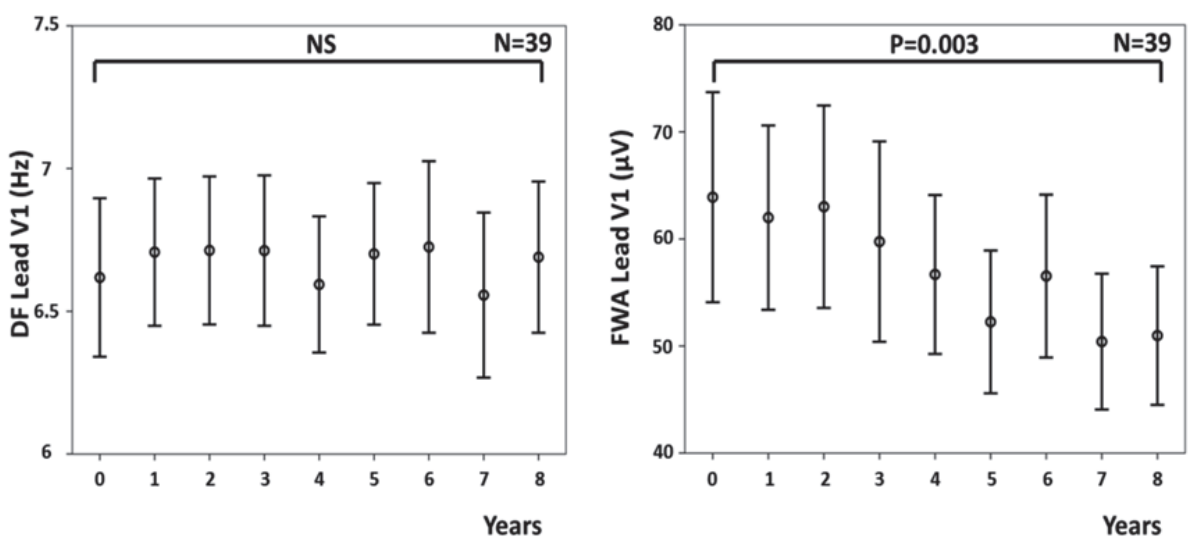

Figure 2: This figure shows the mean dominant frequency (DF) and F-wave amplitude (FWA) in lead V1 in 39 patients with a yearly ECG followed for 8 years. The DF remains relatively stable during the follow-up period. The FWA decreases during the follow-up period.

\section{Part IV: Future directions}

This thesis aims to contribute to the development of a patient tailored rhythm control treatment strategy. Theoretically, rhythm control treatment prevents atrial structural remodelling processes and improves outcome by improving survival and preventing harmful consequences of AF. However, most randomised controlled studies testing rate versus rhythm control strategies did not confirm this hypothesis. ${ }^{32-35}$ In those studies however, only $23-63 \%$ of the rhythm control group was still in sinus rhythm at the end of the study period. This could be explained by cessation of drugs due to adverse drug effects (5\% severe adverse effects in the RACE trial and up to $25 \%$ drug related side effects in the PIAF and AFFIRM trial), or the ineffectiveness of these drugs to maintain sinus rhythm in some patients in an era before catheter ablation. Only the JRHYTHM study showed an advantage of a rhythm control strategy possibly because this study only included paroxysmal, and therefore less remodelled, AF patients. ${ }^{36}$ Therefore, identifying the best rhythm strategy for an individual patient after the diagnosis seems necessary. This thesis helps with the identification of patients likely to benefit from a certain treatment strategy.

It seems worthwhile to study a patient tailored rhythm control therapy, based on the identified clinical and echocardiographic predictors in patients already 
Chapter 9

diagnosed with AF. To this end patients with recently diagnosed AF are of particular interest. New technologies highlighted in chapter 3 that identify patients with AF using their mobile phone or devices such as the "MyDiagnostick" might be useful for this purpose. ${ }^{37,38}$ Besides early detection of AF using these devices, the digitally recorded ECG could be used to determine the DF or FWA and thereby direct therapy in an early stage of the disease. 


\section{References}

1. Morris JJ, Jr., Estes EH, Jr., Whalen RE, Thompson HK, Jr., McIntosh HD. PWave Analysis in Valvular Heart Disease. Circulation 1964;29:242-252.

2. Chirife R, Feitosa GS, Frankl WS. Electrocardiographic detection of left atrial enlargement. Correlation of $\mathrm{P}$ wave with left atrial dimension by echocardiography. British Heart Journal 1975;37:1281-1285.

3. Andrikopoulos GK, Dilaveris PE, Richter DJ, Gialafos EJ, Synetos AG, Gialafos JE. Increased variance of $P$ wave duration on the electrocardiogram distinguishes patients with idiopathic paroxysmal atrial fibrillation. Pacing and Clinical Electrophysiology 2000;23:1127-1132.

4. Potse M, Lankveld TA, Zeemering S, Dagnelie PC, Stehouwer CD, Henry RM, Linnenbank AC, Kuijpers $\mathrm{NH}$, Schotten U. P-wave complexity in normal subjects and computer models. Journal of Electrocardiology 2016;49:545-53.

5. Ferrer A, Sebastian R, Sanchez-Quintana D, Rodriguez JF, Godoy EJ, Martinez L, Saiz J. Detailed Anatomical and Electrophysiological Models of Human Atria and Torso for the Simulation of Atrial Activation. PloS One 2015;10:e0141573.

6. Murthy S, Rizzi P, Mewton N, Strauss DG, Liu CY, Volpe GJ, Marchlinski FE, Spooner P, Berger RD, Kellman P, Lima JA, Tereshchenko LG. Number of Pwave fragmentations on P-SAECG correlates with infiltrated atrial fat. Annals of Noninvasive Electrocardiology 2014;19:114-121.

7. Cochet $\mathrm{H}$, Mouries $\mathrm{A}$, Nivet $\mathrm{H}$, et al. Age, atrial fibrillation, and structural heart disease are the main determinants of left atrial fibrosis detected by delayedenhanced magnetic resonance imaging in a general cardiology population. Journal of Cardiovascular Electrophysiology 2015;26:484-492.

8. Venteclef N, Guglielmi V, Balse E, Gaborit B, Cotillard A, Atassi F, Amour J, Leprince P, Dutour A, Clement K, Hatem SN. Human epicardial adipose tissue induces fibrosis of the atrial myocardium through the secretion of adipofibrokines. European Heart Journal 2015;36:795-805a.

9. Hatem SN, Sanders P. Epicardial adipose tissue and atrial fibrillation. Cardiovascular Research 2014;102:205-213.

10. Allessie MA, de Groot NM, Houben RP, Schotten U, Boersma E, Smeets JL, Crijns HJ. Electropathological substrate of long-standing persistent atrial fibrillation in patients with structural heart disease: longitudinal dissociation. Circulation Arrhythmia and Electrophysiology 2010;3:606-615.

11. Lee S, Sahadevan J, Khrestian CM, Cakulev I, Markowitz A, Waldo AL. Simultaneous Bi-Atrial High Density (510 - 512 electrodes) Epicardial Mapping of Persistent and Long-Standing Persistent Atrial Fibrillation in Patients: New Insights into the Mechanism of Its Maintenance. Circulation 2015;132:210817.

12. Eckstein J, Maesen B, Linz D, Zeemering S, van Hunnik A, Verheule S, Allessie M, Schotten U. Time course and mechanisms of endo-epicardial electrical dissociation during atrial fibrillation in the goat. Cardiovascular Research 2011;89:816-824. 


\section{Chapter 9}

13. de Groot NM, Houben RP, Smeets JL, Boersma E, Schotten U, Schalij MJ, Crijns $\mathrm{H}$, Allessie MA. Electropathological substrate of longstanding persistent atrial fibrillation in patients with structural heart disease: epicardial breakthrough. Circulation 2010;122:1674-1682.

14. Van Gelder IC, Crijns HJ, Van Gilst WH, Verwer R, Lie KI. Prediction of uneventful cardioversion and maintenance of sinus rhythm from directcurrent electrical cardioversion of chronic atrial fibrillation and flutter. The American Journal of Cardiology 1991;68:41-46.

15. Frick $M$, Frykman V, Jensen-Urstad $M$, Ostergren J, Rosenqvist M. Factors predicting success rate and recurrence of atrial fibrillation after first electrical cardioversion in patients with persistent atrial fibrillation. Clinical Cardiology 2001;24:238-244.

16. Scherr D, Khairy P, Miyazaki S, et al. Five-Year Outcome of Catheter Ablation of Persistent Atrial Fibrillation Using Termination of Atrial Fibrillation as a Procedural Endpoint. Circulation Arrhythmia and Electrophysiology 2015;8:1824.

17. Grundvold I, Tveit A, Smith P, Seljeflot I, Abdelnoor M, Arnesen H. The predictive value of transthoracic echocardiographic variables for sinus rhythm maintenance after electrical cardioversion of atrial fibrillation. Results from the CAPRAF study, a prospective, randomized, placebo-controlled study. Cardiology 2008;111:30-35.

18. Boriani G, Diemberger I, Biffi M, Domenichini G, Martignani C, Valzania C, Branzi A. Electrical cardioversion for persistent atrial fibrillation or atrial flutter in clinical practice: predictors of long-term outcome. International Journal of Clinical Practice 2007;61:748-756.

19. Zhuang J, Wang Y, Tang K, Li X, Peng W, Liang C, Xu Y. Association between left atrial size and atrial fibrillation recurrence after single circumferential pulmonary vein isolation: a systematic review and meta-analysis of observational studies. Europace 2012;14:638-645.

20. Rostock T, Salukhe TV, Steven D, et al. Long-term single- and multipleprocedure outcome and predictors of success after catheter ablation for persistent atrial fibrillation. Heart Rhythm 2011;8:1391-1397.

21. Ammar S, Hessling G, Paulik M, Reents $T$, Dillier R, Buiatti A, Semmler V, Kolb C, Haller B, Deisenhofer I. Impact of baseline atrial fibrillation cycle length on acute and long-term outcome of persistent atrial fibrillation ablation. Journal of Interventional Cardiac Electrophysiology 2014;41:253-9.

22. Platonov PG, Corino VD, Seifert M, Holmqvist F, Sornmo L. Atrial fibrillatory rate in the clinical context: natural course and prediction of intervention outcome. Europace 2014;16 Suppl 4:iv110-iv119.

23. Platonov PG, Cygankiewicz I, Stridh M, Holmqvist F, Vazquez R, Bayes-Genis A, McNitt $S$, Zareba W, de Luna AB. Low atrial fibrillatory rate is associated with poor outcome in patients with mild to moderate heart failure. Circulation Arrhythmia and Electrophysiology 2012;5:77-83. 
24. Haissaguerre $M$, Lim KT, Jacquemet V, Rotter $M$, Dang L, Hocini $M$, Matsuo $S$, Knecht $S$, Jais $P$, Virag N. Atrial fibrillatory cycle length: computer simulation and potential clinical importance. Europace 2007;9 Suppl 6:vi64-70.

25. Wijffels MC, Kirchhof CJ, Dorland R, Allessie MA. Atrial fibrillation begets atrial fibrillation. A study in awake chronically instrumented goats. Circulation 1995;92:1954-1968.

26. Jadidi AS, Cochet $\mathrm{H}$, Shah AJ, et al. Inverse relationship between fractionated electrograms and atrial fibrosis in persistent atrial fibrillation: combined magnetic resonance imaging and high-density mapping. Journal of the American College of Cardiology 2013;62:802-812.

27. Swartz MF, Fink GW, Lutz CJ, Taffet SM, Berenfeld O, Vikstrom KL, Kasprowicz K, Bhatta L, Puskas F, Kalifa J, Jalife J. Left versus right atrial difference in dominant frequency, $\mathrm{K}(+)$ channel transcripts, and fibrosis in patients developing atrial fibrillation after cardiac surgery. Heart Rhythm 2009;6:14151422.

28. Bollmann A, Tveit A, Husser D, Stridh M, Sornmo L, Smith P, Olsson SB. Fibrillatory rate response to candesartan in persistent atrial fibrillation. Europace 2008;10:1138-1144.

29. Choudhary MB, Holmqvist F, Carlson J, Nilsson HJ, Roijer A, Platonov PG. Low atrial fibrillatory rate is associated with spontaneous conversion of recentonset atrial fibrillation. Europace 2013;15:1445-1452.

30. Chen YJ, Lee SH, Hsieh MH, Hsiao CJ, Yu WC, Chiou CW, Chen SA. Effects of 17 beta-estradiol on tachycardia-induced changes of atrial refractoriness and cisapride-induced ventricular arrhythmia. Journal of Cardiovascular Electrophysiology 1999;10:587-598.

31. Mohanty S, Mohanty P, Di Biase L, Rong B, Burkhardt D, Gallinghouse JG, Horton R, Sanchez JE, Bailey S, Zagrodzky J, Natale A. Baseline B-type natriuretic peptide: a gender-specific predictor of procedure-outcome in atrial fibrillation patients undergoing catheter ablation. Journal of Cardiovascular Electrophysiology 2011;22:858-865.

32. Van Gelder IC, Hagens VE, Bosker HA, Kingma JH, Kamp O, Kingma T, Said SA, Darmanata JI, Timmermans AJ, Tijssen JG, Crijns HJ. A comparison of rate control and rhythm control in patients with recurrent persistent atrial fibrillation. The New England Journal of Medicine 2002;347:1834-1840.

33. Carlsson J, Miketic S, Windeler J, Cuneo A, Haun S, Micus S, Walter S, Tebbe U. Randomized trial of rate-control versus rhythm-control in persistent atrial fibrillation: the Strategies of Treatment of Atrial Fibrillation (STAF) study. Journal of the American College of Cardiology 2003;41:1690-1696.

34. Hohnloser SH, Kuck KH, Lilienthal J. Rhythm or rate control in atrial fibrillation-Pharmacological Intervention in Atrial Fibrillation (PIAF): a randomised trial. Lancet 2000;356:1789-1794.

35. Wyse DG, Waldo AL, DiMarco JP, Domanski MJ, Rosenberg Y, Schron EB, Kellen JC, Greene HL, Mickel MC, Dalquist JE, Corley SD. A comparison of rate control and rhythm control in patients with atrial fibrillation. The New England Journal of Medicine 2002;347:1825-1833. 


\section{Chapter 9}

36. Ogawa S, Yamashita T, Yamazaki T, et al. Optimal treatment strategy for patients with paroxysmal atrial fibrillation: J-RHYTHM Study. Circulation Journal 2009;73:242-248.

37. Lau JK, Lowres N, Neubeck L, Brieger DB, Sy RW, Galloway CD, Albert DE, Freedman SB. iPhone ECG application for community screening to detect silent atrial fibrillation: a novel technology to prevent stroke. International Journal of Cardiology 2013;165:193-194.

38. Tieleman RG, Plantinga Y, Rinkes D, Bartels GL, Posma JL, Cator R, Hofman C, Houben RP. Validation and clinical use of a novel diagnostic device for screening of atrial fibrillation. Europace 2014;16:1291-1295. 



\section{Summary}

This thesis describes the value of electrocardiographic (ECG) parameters for the prediction of rhythm outcome in patients with atrial fibrillation (AF).

In the first part of this thesis (chapter 2) we described differences in the Pwave characteristics between patients with and without a history of AF. More peaks within the P-wave and a larger terminal part of the P-wave in lead V1 both identified patients more likely to have experienced an AF-episode. More peaks within the $\mathrm{P}$-wave might represent atrial conduction disturbances. Importantly, although more peaks within the P-waves identified patients with a history of AF, especially leads located on the left posterior side of the thorax were of value. This is possibly because these leads mostly represent the conduction in the left atrium as shown in chapter 2.

The second part concentrates on the complexity of the AF-signal derived from the ECG and its ability to predict success of rhythm control strategies in various AF populations. We evaluated a wide variety of ECG-derived complexity parameters to cover both the time and frequency domain. In chapter 4 we showed that a low dominant frequency can predict successful pharmacological cardioversion using flecainide in patients with short-lasting atrial fibrillation. In chapter 5 we confirmed the predictive value of a low dominant frequency in patients with persistent AF referred for electrical cardioversion with 1 year follow-up. In chapter 6 we studied patients with persistent and long-standing persistent AF referred for stepwise catheter ablation. A larger F-wave amplitude was the best performing ECG-parameter for prediction of termination during the index procedure and sinus rhythm after a median follow-up period of 6 years. The predictive value of both the acute and longterm endpoint was confirmed in an external validation cohort. Leftward orientated leads seem to harbour the most predictive value. In all these studies ECG-derived parameters predicted success of rhythm control at least as good as known clinical parameters of sinus rhythm maintenance. Interestingly, the combination of clinical and ECG-derived parameters improved outcome prediction in all studies.

In part three of this thesis we evaluated the influence of clinical characteristics on the dominant frequency and F-wave amplitude (chapter 7). The dominant frequency is lower in patients with anti-arrhythmic drugs, older patients and in female patients. Results for the F-wave amplitude were less consistent but the F-wave amplitude was mostly effected by the duration of the AF-episode. The amplitude became lower with increasing episode duration. In chapter 8 we matched male and female patients referred for electrical cardioversion. A 
lower dominant frequency predicted sinus rhythm maintenance after 1 year follow-up confirming results from chapter $\mathbf{5}$. However, in the subgroup containing female patients the dominant frequency was not able to predict sinus rhythm maintenance. 


\section{Samenvatting}

Dit proefschrift beschrijft de waarde van electrocardiografische (ECG) parameters bij de voorspelling van ritme uitkomst bij patiënten met atriumfibrilleren.

In het eerste gedeelte van het proefschrift (hoofdstuk 2) hebben we verschillen in P-golf karakteristieken tussen patiënten met en zonder atriumfibrilleren (AF) beschreven. Meer pieken in de P-golf en een groter laatste gedeelte van de P-golf in lead V1 identificeerden patiënten die een AF episode doorgemaakt hadden. Meerdere pieken in de P-golf zou kunnen duiden op atriale geleidingsstoornissen. Hoewel het aantal pieken in de P-golf patiënten met een eerdere AF-episode konden identificeren, bleken met name electroden die hoog links op de rug geplaats waren het best te discrimineren tussen de twee groepen. Dit zou kunnen komen doordat deze leads met name links atriale activatie detecteren zoals aangetoond in dit hoofdstuk.

Het tweede gedeelte concentreert zich op het voorspellend vermogen van AFcomplexiteit parameters voor succes van ritme-controle in verschillende populaties van patiënten met AF. We hebben zowel tijds- als frequentiedomein parameters bestudeerd. In hoofdstuk $\mathbf{4}$ hebben we laten zien dat een lage dominante frequentie succesvolle farmacologische cardioversie middels flecaïnide voorspelt. In hoofdstuk $\mathbf{5}$ hebben we de voorspellende waarde van een lage dominante frequentie bevestigd maar nu in een populatie van patiënten met persisterend AF die verwezen werden voor elektrische cardioversie en een jaar gevolgd werden. In hoofdstuk 6 hebben we patiënten met persisterend en langdurig persisterend AF die verwezen werden voor "stepwise" katheter ablatie bestudeerd. Een hogere amplitude van de F-golf was de ECG-parameter die stoppen van AF gedurende de ablatie en sinusritme na mediane follow-up van 6 jaar het beste voorspelde. De voorspellende waarde werd bevestigd in een overeenkomstige populatie in een ander ziekenhuis. Het blijkt dat links georiënteerde leads succes het beste kunnen voorspellen. In al deze studies voorspelden ECG-parameters ritme uitkomst tenminste even goed als bekende klinische parameters. De combinatie van ECG- en klinische parameters verbeterde de voorspellende waarde.

In het derde gedeelte van dit proefschrift hebben we de invloed van karakteristieken van de patiënt op de dominante frequentie en amplitude van de F-golf bestudeerd (hoofdstuk 7). De dominante frequentie is lager bij patiënten die anti-aritmica gebruiken, bij oudere patiënten en vrouwelijke patiënten. Voor de amplitude van de F-golf waren de resultaten minder consistent over de verschillende leads maar bleek de amplitude met name af 
te nemen bij het toenemen van de duur van de AF-episode. In hoofdstuk 8 hebben we mannen en vrouwen met vergelijkbare klinische karakteristieken die verwezen waren voor elektrische cardioversie bestudeerd. Een lagere dominante frequentie voorspelde behoud van sinusritme na 1 jaar net als in hoofdstuk 5. Het bleek echter dat de dominante frequentie dit wel bij de mannelijke populatie kon, maar niet kon voorspellen welke vrouwelijke patiënten sinusritme behielden. 


\section{Valorisation}

\section{Atrial fibrillation}

Atrial fibrillation (AF) is the most common cardiac arrhythmia with an estimated prevalence of approximately $3 \%$ in adults aged 20 years and older. The prevalence of AF increases with advancing age. Furthermore, AF is independently associated with an increase in all-cause mortality and is also associated with increased morbidity, such as heart failure and stroke. As a consequence, a large percentage of healthcare costs is spend on AF.

\section{Diagnosis of incident atrial fibrillation}

Intensified rhythm monitoring of patients with a high likelihood of AF might lead to an earlier diagnosis. With earlier diagnosis of the arrhythmia, adequate treatment such as anticoagulation can be initiated at an earlier stage which might prevent disabling strokes in these patients. The problem is the identification of patients with a high likelihood of developing AF. The first part of this thesis is about the identification of patients with a history of atrial fibrillation. P-wave parameters identified in this thesis are able to classify patients with a previous history of AF more accurately than currently used ECG-parameters. The selection of parameters that are able to discriminate between patients with and without $A F$ is a first step towards an earlier diagnosis of the arrhythmia.

To investigate whether these parameters are indeed able to identify patients with a high likelihood of developing AF, these parameter should be measured in a population based study.

For the implementation of these new P-wave parameters long recordings, without low-pass filters and with a high sampling frequency are required. Furthermore, alternative or additional leads are advocated. At this point, these ECG-recording devices are mainly used for research purposes. The analyses in this thesis were done offline. However, for the implementation in everyday clinical practice the ECG-recording devices should have an integrated software package to analyse the $\mathrm{P}$-wave complexity instantaneously. Further research on software development is required.

\section{Guiding treatment of atrial fibrillation}

For the treatment of AF a rate- and rhythm control strategy can be adopted. If a rhythm control strategy is preferred, the decision on the type of rhythm control strategy (medication, electrical cardioversion, catheter and/or surgical 
ablation or a combination of strategies) is usually based on the preference of the physician and patient. Unfortunately, long-term results of these strategies are disappointing in some strategies (electrical cardioversion) while other strategies (such as ablation) have superior long-term results but with possible complications of the treatment itself.

Identifying patients likely to respond to a certain treatment and therefore selecting the appropriate treatment could enhance efficacy and also prevent unnecessary overtreatment.

We showed that the ECG-derived AF frequency and complexity parameters identified in this thesis can predict success of rhythm control therapy in a wide variety of patient populations. These complexity parameters can be calculated on a standard 10 seconds 12 lead ECG which makes them easy to use in everyday clinical practice. For example, the dominant frequency of the atrial signal can already be calculated on some ECG-recording devices. These parameters still need to prove their ability of selecting the appropriate treatment for an individual patient. Future research should address this question.

\section{Guiding catheter ablation}

In patients with paroxysmal AF pulmonary vein isolation is the established ablation strategy. However, in a subset of patients with persistent AF pulmonary vein isolation alone proves not be sufficient. Selecting the appropriate lesion set in these patients remains a challenging task. Although we showed that endocardial atrial frequencies from the atrial appendages can be measured on the body surface using a standard 12 lead ECG, the spatial resolution of a 12 lead ECG seems to limited for this task. Body surface potential mapping has the advantage of a high spatial resolution and might be able to identify potential ablation targets in a patient. Especially ECG-imaging which incorporates the patient's anatomy might be used for this purpose. Currently studies addressing this question are carried out. 


\section{Dankwoord}

Het proefschrift dat voor u ligt, ligt er door de medewerking en support van een heleboel mensen. Ik wil dit dankwoord gebruiken om een aantal personen in het bijzonder te bedanken.

Prof Schotten, beste Uli, ik ben je dankbaar voor alle input van kennis omtrent de fysiologie en ECG-analyses. Ik waardeerde de lange discussies over de analysemethodes en nieuwe (soms praktisch niet uitvoerbare) ideeën, maar ook het feit dat bij jou de persoon erg centraal staat. Dit uit zich bijvoorbeeld in het feit dat je altijd eerst vroeg hoe het met mij ging in plaats van met al het werk dat eigenlijk nog af moest.

Prof Crijns, beste Harry, je hebt me initieel overdonderd met het aanbod om promotieonderzoek bij je te komen doen. Ik zat namelijk aan het ontbijt in Berlijn tijdens de compensatie van mijn dienstblok. Ik was erg blij met dit telefoontje en heb veel van je geleerd de afgelopen jaren op onderzoeks-, klinisch en ook menselijk vlak waarvoor ik je van harte wil bedanken.

Stef, zonder jou had dit boekje er letterlijk niet in deze vorm gelegen. Ik heb vaak je hulp nodig gehad als het ging om nieuwe analysemethoden (de voor mij lastige wiskundige zaken). Ook konden we lekker brainstormen over nieuwe ideeën en alweer nieuwe ECG-databases die we wellicht konden krijgen. Toen je zelf gepromoveerd was, was het voor mij dan ook duidelijk dat jij mijn copromotor zou moeten worden.

Sandra en Alain bedankt dat jullie hier letterlijk (en ik hoop ook figuurlijk) achter mij willen staan op deze dag.

Ik wil al mijn kamergenoten en medeonderzoekers van de cardiologie (Sandra, Elton, Ömer, Frederique, Mark, Jort, Masih, Caroline, Martijn, Nick, Ruud, Yvonne, Sibel, Sema, Bouke, Mindy, Twan, Arantxa, Ione en Marije) heel erg bedanken voor de leerzame maar vooral ook leuke afgelopen jaren. Ik kijk met veel plezier terug op alle onzin op de kamer, de leuke PhD-uitjes, het borrelen bij Thembi en de vele congressen waar we met $z$ 'n allen naar toe gegaan zijn.

Bob, bedankt voor het inwerken en introduceren van mij op de poli. Het heeft nog lang geduurd voordat de patiënten het niet meer hadden over de charmante dokter met zijn grijze haren.

Bart en Elham, bedankt voor de samenwerking op de OK. Arne met jou kan je altijd lekker discussiëren en filosoferen over de mechanismen die aan AF ten grondslag liggen. 
Pap en mam, ik wil jullie heel erg bedanken voor jullie liefde en opvoeding. Ik sta hier door jullie, jullie zijn mijn grote voorbeeld. Ik vind het heel erg jammer dat jullie dit moment niet meer mee kunnen maken, maar ik weet zeker dat jullie trots zullen zijn. Fleur, ik ben ook erg trots op jou. Ik vind het heel knap hoe $\mathrm{jij}$ het de afgelopen jaren allemaal gedaan hebt.

Alouis en Ria, jullie hebben me in goede en in slechte tijden met open armen ontvangen in huize Heijmans. Dit waardeer ik zeer en hier ben ik jullie zeer dankbaar voor.

Stijn, je beseft het nu nog niet maar jouw komst heeft zoveel geluk in mijn leven gebracht. Ik kan dit niet goed onder woorden brengen. Later als je groot bent moet je dit boekje maar eens lezen (of anders de plaatjes bekijken).

Mieke, je bent als laatste aan de beurt in mijn dankwoord maar dat komt omdat net als in het onderzoek de belangrijkste auteur als laatste genoemd wordt. Jouw steun is ontzettend belangrijk geweest! De laatste jaren waren op zijn zachtst gezegd hectisch te noemen, maar je bleef me steunen. Jouw liefde en begrip hebben ervoor gezorgd dat er nu een kaft om dit boekje zit. 


\section{Curriculum vitae}

Theodorus Antonius Roelf Lankveld was born March $15^{\text {th }} 1985$ in Venray, the Netherlands. He graduated in 2003 from Raayland College secondary school in Venray. He obtained his medical degree in 2010 at the Radboud University Nijmegen, the Netherlands. Between April 2010 and January 2011 he worked at the cardiology department at Viecuri Medical Centre. Between February 2011 and September 2011 he worked at the cardiology department at Maastricht University Medical Centre, Maastricht, the Netherlands. From October 2011 until November 2015 he worked as a research fellow at the department of Cardiology and Physiology under supervision of prof. dr. H.J.G.M. Crijns and prof. dr. U. Schotten. He presented his research at several national and international congresses. He received the first price for oral presentations at the NHRA in 2014 and 2015. The results of his research fellowship are presented in this thesis. At December $1^{\text {st }} 2015$ he started his clinical Cardiology training under supervision of prof. dr. H.J.G.M. Crijns. 


\section{List of publications}

Lankveld TA, Zeemering S, Crijns HJ, Schotten U. The ecg as a tool to determine atrial fibrillation complexity. Heart. 2014;100:1077-1084

Kumar N, Bonizzi P, Pison L, Phan K, Lankveld T, Maessen B, La Meir M, Gelsomino S, Maessen J, Crijns H. Impact of hybrid procedure on $\mathrm{p}$ wave duration for atrial fibrillation ablation. Journal of interventional cardiac electrophysiology. 2015;42:91-9

Kumar N, Bonizzi P, Mafi Rad M, Lankveld T. Left atrial dyssynchrony time measured by tissue doppler imaging to predict atrial fibrillation recurrences after pulmonary vein isolation: Is this a mirage or the panacea? The Anatolian journal of cardiology. 2015;15:123-4

Lau DH, Maesen B, Zeemering S, Kuklik P, van Hunnik A, Lankveld TA, Bidar E, Verheule S, Nijs J, Maessen J, Crijns H, Sanders P, Schotten U. Indices of bipolar complex fractionated atrial electrograms correlate poorly with each other and atrial fibrillation substrate complexity. Heart Rhythm. 2015 Jul;12:1415-23.

Lankveld T, de Vos CB, Limantoro I, Zeemering S, Dudink E, Crijns HJ, Schotten $U$. Systematic analysis of ECG predictors of sinus rhythm maintenance after electrical cardioversion for persistent atrial fibrillation. Heart Rhythm 2016;13:1020-7.

Lankveld T, Zeemering S, Scherr D, Kuklik P, Hoffmann BA, Willems S, Pieske B, Haïssaguerre $M$, Jaïs $P$, Crijns $H J$, Schotten U. Atrial fibrillation complexity parameters derived from surface ECGs predict procedural outcome and longterm follow-up of stepwise catheter ablation for atrial fibrillation. Circ Arrhythmia Electrophysiol 2016;9:e003354

Potse M, Lankveld TA, Zeemering S, Dagnelie PC, Stehouwer CD, Henry RM, Linnenbank AC, Kuijpers NH, Schotten U. P-wave complexity in normal subjects and computer models. J Electrocardiol 2016;49:545-53. 